UNITED STATES DEPARTMENT OF THE INTERIOR

GEOLOGICAL SURVEY

\title{
Semiquantitative emission spectrographic analyses \\ of stream-sediment samples collected in the \\ Livengood and western one-third of the Circle
}

$1^{\circ} \times 3^{\circ}$ quadrangles, Alaska

\author{
By \\ Elizabeth A. Bailey ${ }^{1}$, Gregory K. Lee ${ }^{1}$, \\ and Thomas D. Light 1
}

Open-File Report 87-264

This report is preliminary and has not been reviewed for conformity with U.S. Geological Survey editorial standards and stratigraphic nomenclature. Any use of trade names is for descriptive purposes only and does not imply endorsement by the USGS.

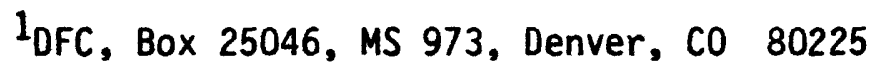




\section{CONTENTS}

Page

Studies Related to BLM...................................... 1

Introduction................................................ 1

Methods of Study $\ldots \ldots \ldots \ldots \ldots \ldots \ldots \ldots \ldots \ldots \ldots \ldots \ldots \ldots \ldots \ldots \ldots \ldots \ldots, 3$

Sample Media........................................... 3

Sample Collection and Analysis........................... 3

Rock Anatysis Storage System (RASS) $\ldots \ldots \ldots \ldots \ldots \ldots \ldots \ldots \ldots \ldots \ldots \ldots, 3$

Description of Data Tables..................................... 3

References Cited.............................................. 4

\section{ILLUSTRATIONS}

Figure 1. Index map of the Livengood and Circle quadrangles, Alaska..... 2

Plate 1. Localities of stream-sediment samples from the Livengood and western $1 / 3$ of the Circle quadrangles, Alaska............ in pocket

\section{TABLES}

Table 1. Limits of determination for spectrographic analysis of stream sediments.......................................... 5

Table 2. Results of analyses of NURE stream-sediment samples from the Livengood and western $1 / 3$ of the Circle quadrangles, Alaska........6 6 


\title{
STUDIES RELATED TO BLM
}

\author{
Bureau of Land Management Recreation Areas
}

The Federal Land Policy and Management Act (Public Law 94-579, October 21,1976 ) requires the U.S. Geological Survey to assess certain areas to determine their mineral resource potential, if any. Results must be made available to the public and be submitted to the President and the Congress. This report presents the results of a geochemical survey of the Livengood and western $1 / 3$ of the Circle quadrangles, Alaska.

\section{INTRODUCTION}

From 1974-1979, the U.S. Atomic Energy Commission (subsequent ly the Energy Resource Development Administration and the Department of Energy) funded the "Hydrogeochemical and Stream Sediment Reconnaissance" project as part of the National Uranium Resource Evaluation (NURE) program to assess uranium resources in the United States (Weaver and others, 1983). In anticipation of mineral resource studies of the Livengood $1^{\circ} \times 3^{\circ}$ quadrangle and the White Mountains National Recreation Area in the Livengood and Circle quadrangles, the U.S. Geological Survey reanalyzed the samples collected in the Livengood and western $1 / 3$ of the Circle $1^{\circ} \times 3^{\circ}$ quadrangles, a total area of about $7,800 \mathrm{mi}^{2}(20,200$ $\mathrm{km}^{2}$ ). The purpose of reanalyzing the NURE samples was to establish a consistent analytical base for integrating the results of more recent sampling, and thereby provide a greater sampling density than would otherwise be available.

The Livengood $1^{\circ} \times 3^{\circ}$ quadrangle is in the east-central part of Alaska from $65^{\circ}$ to $66^{\circ}$ north latitude, and from $147^{\circ}$ to $150^{\circ}$ west longitude. The Circle quadrangle lies due east of the Livengood quadrangle, and the western $1 / 3$ of the quadrangle $\left(146^{\circ}-147^{\circ}\right)$ is included in this report. The E11iot and Dalton Highways traverse the Livengood quadrangle from southeast to northwest. The Steese Highway crosses the southeastern Livengood quadrangle and continues northeasterly across the Circle quadrangle (fig. 1).

The area comprises a northeasterly trending sequence of Precambrian, Paleozoic, and Mesozoic sedimentary and metasedimentary rocks in the northwestern part of the Yukon-Tanana Upland. These rocks cons ist mostly of Cambrian and Ordovician quartzite, argillite, slate, and minor limestone; Ordovician, Silurian, and Devonian limestone, dolostone and chert; JurassicCretaceous conglomerate, graywacke, and quartzite (Chapman and others, 1971). Cretaceous-Tertiary age granitic to granodioritic intrusions and some syenite form topographic highs at Cache Mounta in and Victoria Mountain (Weber and others, 1985). Quaternary loess blankets much of the southern $1 / 3$ of the area, and alluvial deposits fill the major drainage courses. Outcrops are scarce throughout most of the area, except where relief is high. The study area lies within a structurally deformed block between the Tintina fault system to the north and the Denali fault system to the south. The Paleozoic and Mesozoic rocks of the area generally strike northeast and dip northwest or are overturned and dipping steeply southwest (Weber, written commun., 1986).

The topographic relief in the area of this report is about $5,000 \mathrm{ft}$ $(1525 \mathrm{~m})$, with a maximum elevation of $5,286 \mathrm{ft}(1611 \mathrm{~m})$ at Mt. Prindle. The White Mountains form a topographic high on the east and are drained by Beaver Creek on the south, west, and north. The remainder of the Livengood quadrangle lies within the drainages of the Yukon and Tolovana Rivers, separated by the Sawtooth Mountains on the west side of the Livengood quadrangle. The climate of the area is arid to semiarid. The high-latitude tundra prohibits ground-water seepage and enhances surface runoff. 


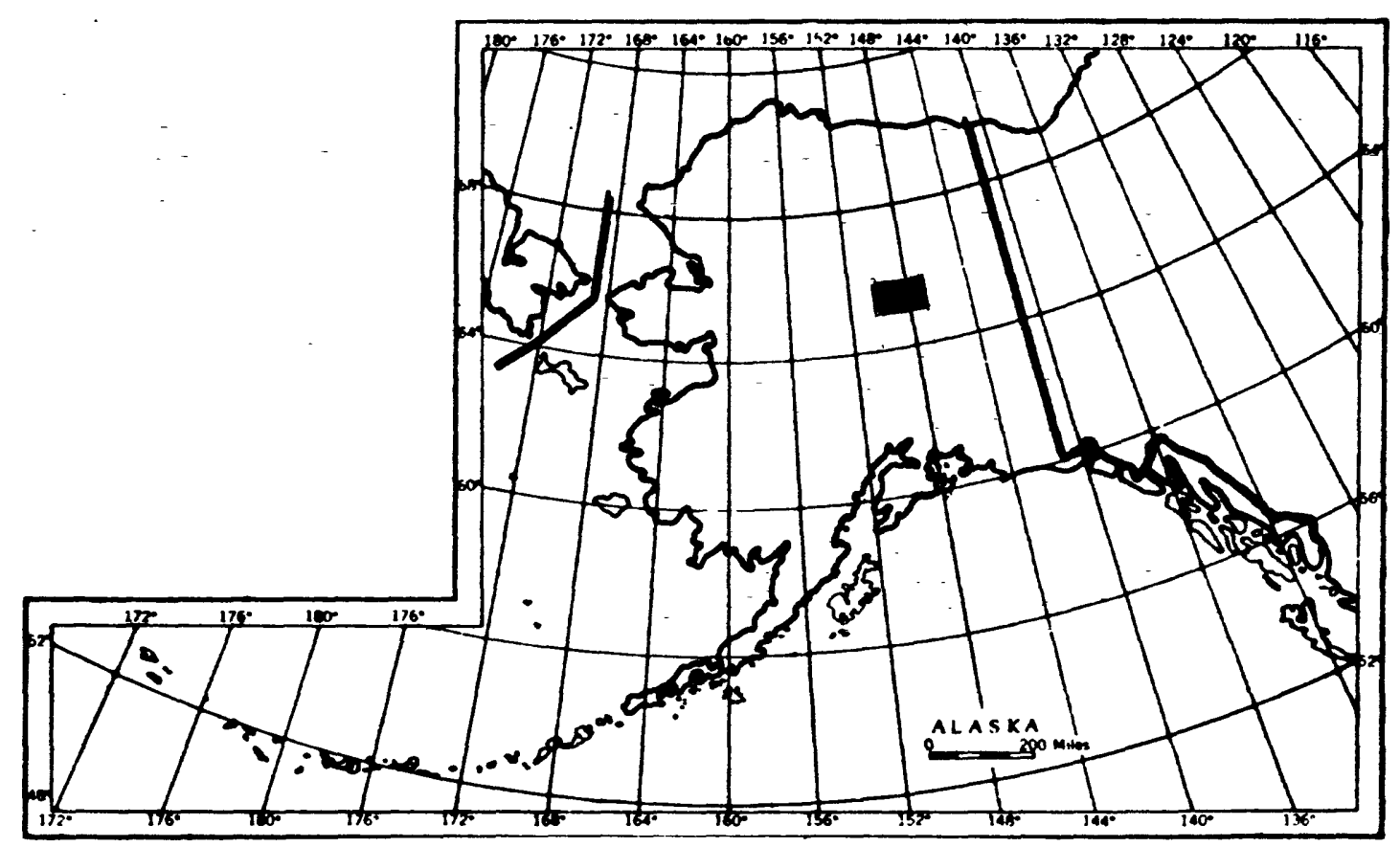

Figure 1. Index map showing the location of the Livengood and western one-third of the Circle quadrangles, Alaska (shaded). 


\section{METHODS OF STUDY}

\section{Sample Media}

Analyses of the stream-sediment samples represent the chemistry of the rock material eroded from the drainage bas in upstream from each sample site. Such information is useful in identifying those basins which contain concentrations of elements that may be related to mineral deposits.

\section{Sample Collection and Analysis}

Samples from 1,010 sites were reanalyzed for this report (plate 1 ). These samples represent fine-grained sediment collected from the active portion of small-order stream drainages. The average sample density for this group of samples is one sample per $7.7 \mathrm{mi}^{2}\left(19.9 \mathrm{~km}^{2}\right)$. Of the original NURE sample set, 5 samples did not have sufficient material remaining for analysis and are not included here.

The minus-100-mesh $(0.15-\mathrm{mm})$ fraction of the stream-sediment samples that remained after analysis by Los Alamos Scientific Laboratory was retained in archival storage. The archived portion of these samples was analyzed for 31 elements using a semiquantitative, direct-current arc emission spectrographic method (Grimes and Marranzino, 1968). The elements analyzed and their lower limits of determination are listed in table 1. Spectrographic results were obtained by visual comparison of spectra derived from the sample against spectra obtained from standards made from pure oxides and carbonates. Standard concentrations are geometrically spaced over any given order of magnitude of concentration as follows: $100,50,20,10$, and so forth. Samples whose concentrations are estimated to fall between those values are assigned values of $70,30,15$, and so forth. The precision of the analytical method is approximately plus or minus one reporting interval at the 83 percent confidence level and plus or minus two reporting intervals at the 96 percent confidence level (Motooka and Grimes, 1976). Analytical data for samples from the Livengood and western 1/3 of the Circle quadrangles are listed in table 2.

\section{ROCK ANALYSIS STORAGE SYSTEM}

Upon completion of all analytical work, the analytical results were entered into a computer-based file called Rock Analysis Storage System (RASS). This data base contains both descriptive geological information and analytical data. Any or all of this information may be retrieved and converted to a binary form (STATPAC) for computerized statistical analysis or publication (VanTrump and Miesch, 1977).

\section{DESCRIPTION OF DATA TABLES}

Table 2 lists the results of analyses for the samples of stream sediment. The data are arranged so that column 1 contains the NURE-assigned sample numbers. These numbers correspond to the numbers shown on the site location map ( $p l a t e ~ 1)$. Columns in which the element headings show the letter "s" below the element symbol are emission spectrographic analyses. A letter "N" in the tables indicates that a given element was looked for but not detected at the lower limit of determination shown for that element in table 1. If an element was observed but was below the lowest reporting value, a "less than" symbol (<) was entered in the tables in front of the lower limit 
of determination. If an element was observed but was above the highest reporting value, a "greater than" symbol ( $>$ ) was entered in the tables in front of the upper limit of determination. Because of the formatting used in the computer program that produced table 2, some of the elements listed in these tables ( $\mathrm{Fe}, \mathrm{Mg}, \mathrm{Ca}, \mathrm{Ti}, \mathrm{Ag}$, and $\mathrm{Be}$ ) carry one or more nonsignificant zeros to the right of the significant digits. The analysts did not determine these elements to the accuracy suggested by the extra zeros.

The spectrographic determinations for $\mathrm{Au}, \mathrm{Cd}$, and $\mathrm{Sb}$ were all below the lower limits of determinations shown in table 1; consequently, the columns for these elements have been deleted from table 2.

\section{REFERENCES CITED}

Chapman, R. M., Weber, F. R., and Taber, Bond, 1971, Preliminary geologic map of the Livengood quadrangle, Alaska: U.S. Geological Survey Open-File Report 71-66, 2 sheets, scale 1:250,000.

Grimes, D. J., and Marranzino, A. P., 1968, Direct-current arc and alternating-current spark emission spectrographic field methods for the semiquantitative analys is of geologic materials: U.S. Geological Survey Circular 591, 6 p.

Motooka, J. M., and Grimes, D. J., 1976, Analytical precision of one-sixth order semiquantitative spectrographic analyses: U.S. Geological Survey Circular 738, 25 p.

VanTrump, George, Jr., and Miesch, A. T., 1977, The U.S. Geological Survey RASS-STATPAC system for management and statistical reduction of geochemical data: Computers and Geosciences, v. 3, p. 475-488.

Weaver, T. A., Broxton, D. E., and Bolivar, S. L., 1983, The Geochemical Atlas of Alaska: Los Alamos National Laboratory Report GJBX-32(83), 49 plates, scale $1: 6,000,000$.

Weber, F. R., Smith, T. E., Ha11, M. H., and Forbes, R. B., 1985, Geologic guide to the Fairbanks-Livengood area, east-central Alaska: Alaska Geological Society Guidebook, 44 p. 
TABLE 1.--Limits of determination for the spectrographic analysis of rocks and stream sediments, based on a 10-mg sample

\begin{tabular}{lcc}
\hline Elements & Lower determination limit & Upper determination limit \\
\hline & Percent & \\
\hline Iron (Fe) & 0.05 & 20 \\
Magnesium (Mg) & .02 & 10 \\
Calcium (Ca) & .05 & 20 \\
Titanium (Ti) & .002 & 1 \\
\hline
\end{tabular}

Parts per million

\begin{tabular}{lrr}
\hline Manganese (Mn) & 10 & \\
Silver (Ag) & 0.5 & 5,000 \\
Arsenic (As) & 200 & 5,000 \\
Gold (Au) & 10 & 10,000 \\
Boron (B) & 10 & 500 \\
Barium (Ba) & 20 & 2,000 \\
Beryllium (Be) & 1 & 5,000 \\
Bismuth (Bi) & 10 & 1,000 \\
Cadmium (Cd) & 20 & 1,000 \\
Cobalt (CO) & 5 & 500 \\
Chromium (Cr) & 10 & 2,000 \\
Copper (Cu) & 5 & 5,000 \\
Lanthanum (La) & 20 & 20,000 \\
Molybdenum (Mo) & 5 & 1,000 \\
Niobium (Nb) & 20 & 2,000 \\
Nickel (Ni) & 5 & 2,000 \\
Lead (Pb) & 10 & 5,000 \\
Antimony (Sb) & 100 & 20,000 \\
Scandium (SC) & 5 & 10,000 \\
Tin (Sn) & 10 & 100 \\
Stront ium (Sr) & 100 & 1,000 \\
Vanadium (V) & 10 & 5,000 \\
Tungsten (W) & 50 & 10,000 \\
Yttrium (Y) & 10 & 2,000 \\
Zinc (Zn) & 200 & 10,000 \\
Zirconium (Zr) & 10 & 1,000 \\
Thorium (Th) & 100 & 2,000 \\
\hline
\end{tabular}


TAELE 2.--RESULTS OF SPECTROGRAPHIC ANALYSES OF NURE STREAM-SEDIMENT SAMPLES FROH THE LIVENGOOD

AND WESTERN $1 / 3$ OF THE CIRCLE QUADRANGLES, ALASKA

[H, not detected; $\langle$, detected but below the liwit of deteraination shown; $\rangle$ deter ained to be greater than the value shown.]

\begin{tabular}{|c|c|c|c|c|c|c|c|c|c|}
\hline Satple & Latitude & Longitude & $\begin{array}{c}\text { Fe-pct. } \\
5\end{array}$ & $\begin{array}{c}\text { Mg-pct. } \\
5\end{array}$ & $\begin{array}{c}\text { Ca-pct. } \\
5\end{array}$ & $\begin{array}{c}\text { Ti-pct. } \\
5\end{array}$ & $\begin{array}{c}\text { Mn-ppm } \\
s\end{array}$ & $\begin{array}{c}\mathrm{Ag}-\mathrm{pp} \text { i } \\
5\end{array}$ & $\begin{array}{l}\text { As-ppo } \\
5\end{array}$ \\
\hline 421951 & 653252 & $148 \quad 26 \quad 17$ & 5.00 & 1.00 & 3.0 & 1.000 & 500 & N & N \\
\hline 421952 & 653252 & $14810 \quad 6$ & 7.00 & 1.50 & 3.0 & 1.000 & 700 & N & $N$ \\
\hline 421954 & 653253 & $148 \quad 512$ & 7.00 & 2.00 & 5.0 & 1.000 & 1,000 & $N$ & N \\
\hline 421955 & 652639 & 1471416 & 5.00 & 2.00 & 3.0 & .700 & 1,500 & $N$ & N \\
\hline 421956 & 652645 & $14712 \quad 2$ & 7.00 & 1.50 & 3.0 & .700 & 700 & $N$ & $N$ \\
\hline 421958 & 652639 & $147 \quad 6 \quad 10$ & 7.00 & 1.50 & 3.0 & 1.000 & 500 & N & N \\
\hline 421959 & 652330 & $147 \quad 515$ & 5.00 & 1.50 & 3.0 & .700 & 300 & $N$ & $N$ \\
\hline 421960 & 652132 & 14753 & 7.00 & 1.50 & 3.0 & 1.000 & 700 & $N$ & $N$ \\
\hline 421961 & 651824 & 147336 & 5.00 & 1.50 & 2.0 & 1.000 & 500 & $N$ & N \\
\hline 421962 & $6517 \quad 17$ & $147 \quad 6 \quad 10$ & 7.00 & 1.50 & 3.0 & 1.000 & 700 & $N$ & N \\
\hline 421963 & 651458 & $147 \quad 244$ & 10.00 & 1.50 & 1.5 & 1.000 & 1,000 & $N$ & $N$ \\
\hline 421964 & 651144 & 147312 & 7.00 & 1.50 & 2.0 & 1.000 & 1,000 & $N$ & $\mathrm{H}$ \\
\hline 421965 & 65118 & $147 \quad 248$ & 7.00 & 1.50 & 1.5 & 1.000 & 700 & N & N \\
\hline 421966 & 651132 & 1475415 & 7.00 & 1.50 & 2.0 & 1.000 & 500 & $N$ & $N$ \\
\hline 421967 & 65160 & $14755 \quad 18$ & 5.00 & 1.50 & 2.0 & 1.000 & 500 & $N$ & N \\
\hline 421968 & 651924 & $14751 \quad 42$ & 7.00 & 1.50 & 2.0 & 1.000 & 1,000 & N & $N$ \\
\hline 421969 & 652943 & 1475559 & 5.00 & 1.50 & 2.0 & 1.000 & 500 & $N$ & $N$ \\
\hline 421970 & 652934 & 1475013 & 10.00 & 2.00 & 2.0 & 1.000 & 3,000 & $N$ & $N$ \\
\hline 421971 & 653017 & 1474918 & 7.00 & 2.00 & 3.0 & 1.000 & 1,500 & $H$ & N \\
\hline 421972 & $6530 \quad 11$ & $147 \quad 25 \quad 2$ & 5.00 & 1.50 & 2.0 & 1.000 & 500 & $N$ & H \\
\hline 421973 & 652934 & $147 \quad 24 \quad 18$ & 7.00 & 1.50 & 2.0 & 1.000 & 700 & N & $H$ \\
\hline 421974 & 652859 & 1471439 & 5.00 & 1.50 & 2.0 & .700 & 500 & $N$ & N \\
\hline 421975 & 652846 & $147 \quad 849$ & 7.00 & 1.50 & 2.0 & .700 & 500 & $N$ & H \\
\hline 421977 & 652912 & 14732 & 7.00 & 1.50 & 3.0 & .700 & 700 & $N$ & $N$ \\
\hline 421979 & 653146 & 14715 & 7.00 & 2.00 & 3.0 & 1.000 & 1,000 & $N$ & N \\
\hline 421980 & 653214 & $147 \quad 410$ & 5.00 & 2.00 & 3.0 & .700 & 700 & $N$ & $N$ \\
\hline 421901 & 653214 & 147535 & 5.00 & 1.50 & 2.0 & .700 & 500 & N & N \\
\hline 421982 & 653230 & 1471218 & 7.00 & 2.00 & 5.0 & 1.000 & 1,000 & $H$ & N \\
\hline 421983 & 653222 & $14715 \quad 0$ & 7.00 & 2.00 & 5.0 & 1.000 & 1,500 & $N$ & $N$ \\
\hline 421986 & 653154 & 1472840 & 7.00 & 1.50 & 2.0 & .700 & 1,000 & .5 & $N$ \\
\hline 421987 & 653159 & 1473656 & 3.00 & 1.00 & 1.5 & .700 & 500 & $N$ & N \\
\hline 421988 & 65327 & $14756 \quad 9$ & 3.00 & 1.50 & 2.0 & 1.000 & 300 & $N$ & $N$ \\
\hline 421989 & 653127 & 1475949 & 5.00 & 1.50 & 1.0 & .700 & 300 & 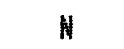 & N \\
\hline 421990 & 65301 & $148 \quad 30 \quad 2$ & 7.00 & 2.00 & 2.0 & 1.000 & 1,000 & $N$ & $N$ \\
\hline 421991 & 652835 & 1482035 & 7.00 & 1.50 & 2.0 & 1.000 & 1,500 & $N$ & N \\
\hline 421992 & $6529 \quad 8$ & $148 \quad 1547$ & 5.00 & 1.50 & 2.0 & .700 & 500 & $N$ & N \\
\hline 421993 & 652829 & $148 \quad 1355$ & 5.00 & 1.50 & 1.5 & .700 & 300 & 6.5 & H \\
\hline 421994 & 652952 & 14893 & 7.00 & 1.50 & 1.0 & 1.000 & 700 & $N$ & $N$ \\
\hline 421995 & 652335 & 1475537 & 7.00 & 2.00 & 3.0 & 1.000 & 700 & N & $n$ \\
\hline 421996 & 652336 & 1473149 & 7.00 & 1.50 & 2.0 & 1.000 & 700 & $N$ & $N$ \\
\hline 421997 & 652435 & $148 \quad 839$ & 5.00 & 1.50 & 5.0 & .700 & 1,000 & 5.0 & $N$ \\
\hline 421999 & 652435 & 1481036 & 10.00 & 5.00 & 7.0 & $>1.000$ & 1,500 & $N$ & $N$ \\
\hline 421999 & 652934 & 1482811 & 7.00 & 1.50 & 3.0 & .700 & 1,000 & N & N \\
\hline 422000 & 6515 & 1472917 & 5.00 & 1.50 & 3.0 & .700 & 500 & .7 & H \\
\hline 422001 & $65 \quad 054$ & $14728 \quad 8$ & 5.00 & 1.50 & 1.5 & .700 & 1,000 & N & $N$ \\
\hline
\end{tabular}


TABLE 2.--RESULTS OF SPECTROGRAPHIC AMALYSES OF NURE STREAM-SEDIMENT SAMPLES FROM THE LIVENGOOD

AND WESTERH $1 / 3$ OF THE CIRCLE QUADRANGLES, ALASKA--ContinUed

\begin{tabular}{|c|c|c|c|c|c|c|c|c|c|c|}
\hline Sample & $\begin{array}{c}\text { B-pp } \\
5\end{array}$ & $\begin{array}{c}\text { Ba-ppil } \\
\mathrm{s}\end{array}$ & $\begin{array}{c}\text { Be-ppi } \\
5\end{array}$ & $\begin{array}{c}\text { Bi-ppil } \\
5\end{array}$ & $\begin{array}{c}\text { Co-pp⿴囗十 } \\
5\end{array}$ & $\begin{array}{c}\mathrm{Cr}-\mathrm{pp} \text { ili } \\
5\end{array}$ & $\begin{array}{c}\text { Cu-ppm } \\
s\end{array}$ & $\begin{array}{c}\text { La-ppol } \\
5\end{array}$ & $\begin{array}{c}\text { Mo-ppm } \\
5\end{array}$ & $\begin{array}{c}N b-p p \text { m } \\
5\end{array}$ \\
\hline 421951 & 70 & 1,000 & $\langle 1.0$ & $N$ & 15 & 200 & 30 & 30 & N & $<20$ \\
\hline 421952 & 70 & 2,000 & $\langle 1.0$ & $N$ & 15 & 200 & 30 & 50 & $N$ & $<20$ \\
\hline 421954 & 50 & 1,500 & $\langle 1.0$ & $N$ & 20 & 150 & 30 & 20 & $N$ & $N$ \\
\hline 421955 & 50 & 1,500 & 1.0 & $N$ & 15 & 1,000 & 30 & 30 & $N$ & H \\
\hline 421956 & 70 & 1,500 & $\langle 1.0$ & $N$ & 15 & 200 & 50 & 30 & N & $N$ \\
\hline 421958 & 70 & 1,500 & 11.0 & $N$ & 20 & 300 & 50 & 30 & $N$ & $<20$ \\
\hline 421959 & 50 & 1,500 & $\langle 1.0$ & $N$ & 15 & 150 & 30 & 50 & $N$ & $<20$ \\
\hline 421960 & 70 & 2,000 & $\langle 1.0$ & $N$ & 15 & 300 & 50 & 50 & $N$ & $<20$ \\
\hline 421961 & 70 & 1,500 & 1.5 & $N$ & 10 & 200 & 20 & 70 & $N$ & $<20$ \\
\hline 421962 & 70 & 2,000 & $\langle 1.0$ & $N$ & 15 & 200 & 20 & 50 & N & $<20$ \\
\hline 421963 & 100 & 2,000 & 1.5 & $N$ & 15 & 200 & 20 & 70 & $N$ & $N$ \\
\hline 421964 & 70 & 2,000 & $\langle 1.0$ & $N$ & 15 & 200 & 15 & 70 & $N$ & $<20$ \\
\hline 421965 & 100 & 1,000 & 1.0 & $N$ & 15 & 200 & 30 & 70 & $N$ & $<20$ \\
\hline 421966 & 70 & 1,500 & $\langle 1.0$ & $N$ & 15 & 200 & 20 & 50 & $N$ & $<20$ \\
\hline 421967 & 50 & 200 & 1.0 & $N$ & 10 & 200 & 15 & 50 & $N$ & $<20$ \\
\hline 421968 & 100 & 2,000 & 1.0 & $\mathbf{N}$ & 15 & 200 & 20 & 70 & $N$ & $<20$ \\
\hline 421969 & 100 & 700 & $\langle 1.0$ & $N$ & 15 & 300 & 20 & 50 & $N$ & $<20$ \\
\hline 421970 & 100 & 1,500 & $\{1.0$ & $\mathrm{H}$ & 20 & 200 & 30 & 30 & $N$ & $<20$ \\
\hline 421971 & 100 & 1,500 & 1.0 & $N$ & 20 & 200 & 20 & 50 & $N$ & $<20$ \\
\hline 421972 & 50 & 1,000 & $\langle 1.0$ & $N$ & 15 & 200 & 15 & 50 & $N$ & $N$ \\
\hline 421973 & 100 & 1,500 & 1.0 & $N$ & 20 & 200 & 30 & 50 & $N$ & $<20$ \\
\hline 421974 & 70 & 1,500 & 1.0 & $N$ & 15 & 150 & 20 & 50 & $N$ & $N$ \\
\hline 421975 & 50 & 1,500 & $\$ 1.0$ & $N$ & 15 & 200 & 20 & 30 & N & $N$ \\
\hline 421977 & 70 & 2,000 & 1.0 & $N$ & 15 & 200 & 30 & 30 & $N$ & $N$ \\
\hline 421979 & 100 & 1,500 & 1.0 & $N$ & 15 & 300 & 30 & 50 & $N$ & $<20$ \\
\hline 421980 & 70 & 2,000 & 1.0 & $N$ & 15 & 200 & 20 & 50 & $N$ & $<20$ \\
\hline 421981 & 50 & 1,000 & 11.0 & $N$ & 10 & 200 & 20 & 50 & N & $N$ \\
\hline 421982 & 70 & 2,000 & 1.0 & $N$ & 20 & 300 & 30 & 50 & $N$ & $N$ \\
\hline 421983 & 50 & 1,500 & 1.0 & $N$ & 20 & 200 & 20 & 50 & $N$ & $N$ \\
\hline 421986 & 100 & 1,500 & 2.0 & $N$ & 20 & 200 & 30 & 50 & N & $<20$ \\
\hline 421987 & 70 & 700 & 2.0 & $N$ & 15 & 100 & 15 & 50 & $N$ & $N$ \\
\hline 421988 & 70 & 1,000 & 3.0 & $N$ & 10 & 100 & 15 & 70 & N & $<20$ \\
\hline 421989 & 50 & 700 & 2.0 & $N$ & 10 & 150 & 15 & 70 & 45 & $<20$ \\
\hline 421990 & 70 & 1,500 & 1.0 & $\mathbf{N}$ & 20 & 200 & 20 & 70 & $N$ & $<20$ \\
\hline 421991 & 70 & 1,500 & 1.0 & $<10$ & 20 & 150 & 30 & 100 & $N$ & $<20$ \\
\hline 421992 & 70 & 1,500 & 1.0 & $N$ & 15 & 200 & 20 & 50 & $N$ & $<20$ \\
\hline 421993 & 50 & 1,000 & 1.0 & $N$ & 15 & 150 & 20 & 70 & $N$ & $<20$ \\
\hline 421994 & 70 & 1,500 & 61.0 & $N$ & 20 & 300 & 20 & 70 & $<5$ & $<20$ \\
\hline 421995 & 70 & 2,000 & 11.0 & $N$ & 20 & 200 & 20 & 50 & $N$ & $<20$ \\
\hline 421996 & 100 & 2,000 & 1.0 & $N$ & 15 & 200 & 20 & 50 & $N$ & $\langle 20$ \\
\hline 421997 & 20 & 2,000 & $(1.0$ & $N$ & 15 & 100 & 20 & 30 & $N$ & $N$ \\
\hline 421998 & 20 & 3,000 & $\langle 1.0$ & $\ddot{N}$ & 20 & 500 & 30 & 50 & N & $<20$ \\
\hline 421999 & 50 & 1,500 & 1.0 & $N$ & 15 & 200 & 20 & 50 & $N$ & $<20$ \\
\hline 422000 & 50 & 1,500 & $\langle 1.0$ & $N$ & 10 & 200 & 30 & 30 & $N$ & $N$ \\
\hline 422001 & 70 & 1,500 & 11.0 & $N$ & 15 & 150 & 20 & 50 & N & N \\
\hline
\end{tabular}


TABLE 2.--RESULTS OF SPECTROgRAPHIC AMALYSES OF NURE STREAM-SEDIMEHT SAMPLES FROM THE LIVENGOOD

AND WESTERN $1 / 3$ OF THE CIRCLE QUADRANGLES, ALASKA-Continued

\begin{tabular}{|c|c|c|c|c|c|c|c|c|c|c|c|}
\hline Sa解le & $\begin{array}{c}\mathrm{Ni}-\mathrm{pp} \text { ( } \\
5\end{array}$ & $\begin{array}{c}\mathrm{Pb}-\mathrm{pp} \\
5\end{array}$ & $\begin{array}{c}\text { Sc-pp } \\
5\end{array}$ & $\begin{array}{c}5 n-p p \text { i } \\
5\end{array}$ & $\begin{array}{c}S r-p p \text { i } \\
s\end{array}$ & $\begin{array}{c}V-p p \text { i } \\
5\end{array}$ & $\begin{array}{c}W-p p \text { i } \\
5\end{array}$ & $\begin{array}{c}Y \text {-pp } \\
5\end{array}$ & $\begin{array}{c}\text { In-ppi } \\
5\end{array}$ & $\begin{array}{c}2 r-p \rho \operatorname{cin} \\
5\end{array}$ & $\begin{array}{c}\text { Th-ppli } \\
s\end{array}$ \\
\hline 421951 & 50 & 15 & 10 & $N$ & 200 & 200 & H & 30 & $N$ & 300 & $N$ \\
\hline 421952 & 30 & 20 & 20 & $H$ & 300 & 150 & $N$ & 50 & $N$ & 500 & $N$ \\
\hline 421954 & 30 & 20 & 30 & N & 500 & 150 & N & 50 & $N$ & 300 & $H$ \\
\hline 421955 & 70 & 15 & 20 & $N$ & 200 & 150 & $N$ & 30 & $<200$ & 300 & H \\
\hline 421956 & 30 & 30 & 20 & $N$ & 300 & 150 & $H$ & 30 & $N$ & 200 & $N$ \\
\hline 421958 & 50 & 30 & 20 & $N$ & 300 & 150 & $N$ & 30 & $N$ & 300 & $N$ \\
\hline 421959 & 30 & 30 & 15 & N & 200 & 150 & $H$ & 30 & $N$ & 300 & $N$ \\
\hline 421960 & 30 & 30 & 20 & N & 300 & 200 & N & 30 & $N$ & 300 & $N$ \\
\hline 421961 & 20 & 50 & 20 & $<10$ & 200 & 100 & $N$ & 70 & $N$ & 500 & $H$ \\
\hline 421962 & 20 & 20 & 20 & $<10$ & 300 & 150 & H & 50 & $N$ & 500 & $N$ \\
\hline 421963 & 30 & 30 & 20 & 10 & 150 & 150 & $N$ & 50 & $N$ & 500 & H \\
\hline 421964 & 30 & 30 & 20 & $H$ & 300 & 150 & H & 50 & $N$ & 700 & $N$ \\
\hline 421965 & 30 & 30 & 20 & $N$ & 150 & 100 & $N$ & 50 & $\mathrm{~N}$ & 300 & $N$ \\
\hline 421966 & 30 & 20 & 20 & $N$ & 200 & 100 & N & 50 & $\mathrm{H}$ & 500 & $H$ \\
\hline 421967 & 30 & 20 & 20 & $N$ & 300 & 150 & N & 30 & $N$ & 300 & $N$ \\
\hline 421968 & 30 & 30 & 20 & $N$ & 200 & 100 & N & 50 & $N$ & 300 & $H$ \\
\hline 421969 & 30 & 20 & 20 & $N$ & 150 & 100 & $H$ & 50 & $H$ & 300 & $H$ \\
\hline 421970 & 50 & 30 & 20 & $N$ & 200 & 150 & $N$ & 50 & $N$ & 500 & $H$ \\
\hline 421971 & 50 & 30 & 20 & $N$ & 200 & 150 & $N$ & 50 & $N$ & 500 & H \\
\hline 421972 & 30 & 20 & 15 & $N$ & 200 & 100 & $H$ & 30 & $N$ & 200 & $N$ \\
\hline 421973 & 30 & 30 & 20 & $N$ & 200 & 200 & $N$ & 50 & $N$ & 300 & $N$ \\
\hline 421974 & 30 & 20 & 15 & $N$ & 200 & 150 & $H$ & 30 & $N$ & 500 & $N$ \\
\hline 421975 & 30 & 30 & 20 & $N$ & 150 & 200 & $H$ & 30 & $N$ & 200 & $N$ \\
\hline 421977 & 30 & 20 & 20 & $N$ & 200 & 200 & $N$ & 30 & $N$ & 500 & $N$ \\
\hline 421979 & 50 & 30 & 20 & H & 300 & 200 & $N$ & 50 & $H$ & 500 & H \\
\hline 421980 & 30 & 20 & 20 & H & 300 & 200 & $N$ & 30 & $N$ & 300 & $N$ \\
\hline 421981 & 30 & 20 & 15 & $N$ & 200 & 150 & $N$ & 30 & $N$ & 500 & H \\
\hline 421982 & 30 & 20 & 20 & $N$ & 300 & 150 & $H$ & 50 & $N$ & 500 & $N$ \\
\hline 421993 & 30 & 20 & 20 & $N$ & 300 & 150 & $N$ & 30 & $N$ & 500 & $N$ \\
\hline 421986 & 50 & 50 & 20 & $\langle 10$ & 200 & 150 & $N$ & 50 & 200 & 300 & $N$ \\
\hline 421987 & 20 & 20 & 15 & 15 & 150 & 100 & $N$ & 50 & $N$ & 200 & $N$ \\
\hline 421988 & 20 & 30 & 15 & 10 & 150 & 150 & $N$ & 50 & $<200$ & 300 & $N$ \\
\hline 421989 & 20 & 50 & 15 & 15 & 100 & 150 & $N$ & 50 & $H$ & 300 & $N$ \\
\hline 421990 & 30 & 50 & 20 & 10 & 200 & 200 & $N$ & 70 & $N$ & 500 & $N$ \\
\hline 421991 & 30 & 50 & 20 & 70 & 200 & 150 & $N$ & 70 & $N$ & 700 & $N$ \\
\hline 421992 & 30 & 20 & 20 & N & 300 & 200 & $N$ & 30 & $N$ & 300 & $N$ \\
\hline 421993 & 30 & 30 & 20 & $N$ & 200 & 150 & $N$ & 30 & $H$ & 200 & $N$ \\
\hline 421994 & 30 & 30 & 20 & $N$ & 150 & 150 & $N$ & 30 & $N$ & 300 & $N$ \\
\hline 421995 & 30 & 20 & 20 & N & 300 & 150 & N & 50 & $N$ & 300 & $H$ \\
\hline 421996 & 30 & 20 & 15 & $N$ & 200 & 150 & $N$ & 50 & N & 500 & N \\
\hline 421997 & 50 & 10 & 10 & $H$ & 200 & 150 & $N$ & 20 & $<200$ & 150 & $N$ \\
\hline 421998 & 50 & 10 & 30 & $N$ & 700 & 300 & $N$ & 50 & $N$ & 150 & $H$ \\
\hline 421999 & 30 & 20 & 15 & $N$ & 300 & 150 & $N$ & 30 & $N$ & 300 & $N$ \\
\hline 422000 & 30 & 15 & 15 & $N$ & 300 & 150 & $N$ & 30 & $<200$ & 300 & $N$ \\
\hline 422001 & 30 & 15 & 15 & $N$ & 200 & 150 & $N$ & 30 & $N$ & 300 & $N$ \\
\hline
\end{tabular}




\begin{tabular}{|c|c|c|c|c|c|c|c|c|c|}
\hline Sample & Latitude & Longitude & $\begin{array}{c}\text { Fe-pct. } \\
5\end{array}$ & $\begin{array}{c}M_{g}-p c t . \\
s\end{array}$ & $\begin{array}{c}\text { Ca-pct. } \\
\text { s }\end{array}$ & $\begin{array}{c}\text { Ti-pct. } \\
s\end{array}$ & $\begin{array}{c}\text { Mn-pp } \\
5\end{array}$ & $\begin{array}{c}\mathrm{Ag}-p p \\
\mathbf{s}\end{array}$ & $\begin{array}{c}\text { As-pp } \\
s\end{array}$ \\
\hline 422002 & $65 \quad 039$ & 1471245 & 5.00 & 2.00 & 5.0 & 1.000 & 1,000 & N & $N$ \\
\hline 422003 & $65 \quad 16$ & 147161 & 10.00 & 1.50 & .7 & 1.000 & 1,000 & $<.5$ & 200 \\
\hline 422004 & 65318 & 1473035 & 10.00 & 2.00 & 1.5 & .700 & 1,500 & $N$ & $N$ \\
\hline 422005 & $65 \quad 4 \quad 14$ & 1472453 & 5.00 & 2.00 & 3.0 & 1.000 & 700 & N & $N$ \\
\hline 422006 & $65 \quad 557$ & 1472437 & 7.00 & 2.00 & 5.0 & 1.000 & 1,500 & N & $N$ \\
\hline 422007 & $65 \quad 625$ & 1472854 & 10.00 & 3.00 & 5.0 & 1.000 & 2,000 & $N$ & $N$ \\
\hline 422008 & $65 \quad 817$ & 1472850 & 7.00 & 2.00 & 3.0 & 1.000 & 500 & N & $N$ \\
\hline 422009 & $65 \quad 024$ & 1473851 & 7.00 & 1.50 & 3.0 & 1.000 & 700 & $N$ & N \\
\hline 422010 & 65211 & 1473538 & 7.00 & 1.50 & 3.0 & 1.000 & 700 & N & $N$ \\
\hline 422011 & 65356 & 1473955 & 5.00 & 1.50 & 5.0 & 1.000 & 700 & $N$ & $N$ \\
\hline 422012 & 6570 & $14746 \quad 23$ & 5.00 & 1.50 & 2.0 & .700 & 500 & N & $N$ \\
\hline 422013 & $65 \quad 937$ & 1475711 & 5.00 & 2.00 & 2.0 & 1.000 & 500 & $N$ & $N$ \\
\hline 422015 & 651121 & 1475829 & 1.50 & .20 & .3 & .150 & 200 & 6.5 & $N$ \\
\hline 422016 & 651154 & $148 \quad 420$ & 2.00 & 1.50 & .7 & .300 & 200 & N & $N$ \\
\hline 422017 & 651445 & $148 \quad 831$ & 5.00 & 1.50 & .7 & .700 & 300 & N & $N$ \\
\hline 422019 & 651452 & 148741 & 7.00 & 2.00 & 2.0 & 1.000 & 700 & $N$ & N \\
\hline 422020 & $6516 \quad 4$ & $148 \quad 633$ & 2.00 & 1.00 & .7 & .500 & 300 & N & $N$ \\
\hline 422022 & 652039 & 1491745 & 10.00 & 2.00 & 2.0 & 1.000 & 700 & $N$ & $N$ \\
\hline 422023 & 652222 & $148 \quad 16 \quad 15$ & 5.00 & 1.00 & 2.0 & .300 & 200 & $N$ & $N$ \\
\hline 422024 & $6524 \quad 42$ & $14814 \quad 27$ & 7.00 & 2.00 & 3.0 & 1.000 & 700 & $N$ & $N$ \\
\hline 422025 & 6510 & 1473731 & 2.00 & 1.00 & 2.0 & .300 & 1,500 & N & $N$ \\
\hline 422026 & 65256 & 1473932 & 15.00 & 3.00 & 1.5 & 1.000 & 2,000 & $N$ & $N$ \\
\hline 422027 & $65 \quad 454$ & $14742 \quad 43$ & 7.00 & 1.50 & 3.0 & 1.000 & 1,000 & 10.0 & $N$ \\
\hline 422028 & 6569 & $14744 \quad 43$ & 7.00 & 2.00 & 1.5 & 1.000 & 1,000 & $N$ & $N$ \\
\hline 422029 & 65756 & 1475049 & 10.00 & 2.00 & 5.0 & $>1.000$ & 1,500 & .5 & $N$ \\
\hline 422030 & 6596 & 1475123 & 5.00 & 1.50 & 2.0 & .700 & 700 & $N$ & $N$ \\
\hline 422031 & 651112 & 1475949 & 10.00 & 2.00 & 3.0 & 1.000 & 2,000 & $N$ & $N$ \\
\hline 422032 & 65114 & $148 \quad 244$ & 7.00 & 1.50 & 3.0 & 1.000 & 1,000 & .7 & 200 \\
\hline 422033 & 651112 & $148 \quad 454$ & 20.00 & 1.50 & 3.0 & .500 & 5,000 & $N$ & 3,000 \\
\hline 422034 & 653318 & 1491932 & 10.00 & 2.00 & 5.0 & 1.000 & 2,000 & N & $N$ \\
\hline 422035 & $6529 \quad 12$ & $148 \quad 014$ & 7.00 & 1.50 & 2.0 & 1.000 & 700 & $N$ & $N$ \\
\hline 422036 & $6527 \quad 14$ & 1474556 & 5.00 & 1.50 & 3.0 & 1.000 & 1,000 & $N$ & $N$ \\
\hline 422037 & 652711 & 1473949 & 5.00 & 2.00 & 5.0 & 1.000 & 700 & $N$ & $N$ \\
\hline 422038 & 652745 & $14734 \quad 8$ & 5.00 & 2.00 & 3.0 & 1.000 & 700 & $N$ & $N$ \\
\hline 422039 & 652629 & 1472712 & 5.00 & 2.00 & 5.0 & 1.000 & 700 & $N$ & $N$ \\
\hline 422040 & 65938 & 1475438 & 5.00 & 2.00 & 3.0 & 1.000 & 1,000 & $N$ & $N$ \\
\hline 422041 & 651448 & 1475241 & 7.00 & 1.50 & 2.0 & 1.000 & 1,000 & $N$ & $N$ \\
\hline 422042 & 65216 & 1475115 & 7.00 & 2.00 & 5.0 & 1.000 & 1,000 & N & $N$ \\
\hline 422043 & 652948 & 1475327 & 7.00 & 1.50 & 3.0 & 1.000 & 1,000 & N & $N$ \\
\hline 422044 & 652944 & $14740 \quad 5$ & 10.00 & 2.00 & 3.0 & 1.000 & 1,500 & $N$ & $N$ \\
\hline 422045 & 653222 & 1474340 & 7.00 & 1.50 & 2.0 & 1.000 & 1,000 & N & $N$ \\
\hline 422047 & 653158 & 1474919 & 7.00 & 1.50 & 5.0 & 1.000 & 1,500 & N & $N$ \\
\hline 422048 & 652652 & $148 \quad 13 \quad 32$ & 10.00 & 2.00 & 1.5 & 1.000 & 1,500 & $N$ & $N$ \\
\hline 422049 & 652659 & $148 \quad 217$ & 10.00 & 2.00 & 2.0 & 1.000 & 2,000 & $N$ & $N$ \\
\hline 422050 & 652245 & 147545 & 7.00 & 1.50 & 2.0 & 1.000 & 1,000 & $N$ & N \\
\hline
\end{tabular}


TABLE 2.--RESULTS OF SPECTROGRAPHIC AMALYSES OF NURE STREAM-SEDIMENT SAMPLES FROH THE LIVENGOOD

AND WESTERN $1 / 3$ OF THE CIRCLE QUADRANGLES, ALASKA--Continued

\begin{tabular}{|c|c|c|c|c|c|c|c|c|c|c|}
\hline Sample & $\begin{array}{c}\text { B-ppm } \\
5\end{array}$ & $\begin{array}{c}\text { Ba-pp } \\
5\end{array}$ & $\begin{array}{c}\text { Ee-ppo } \\
5\end{array}$ & 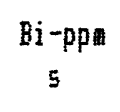 & $\begin{array}{c}\text { [0-pp\# } \\
\mathbf{5}\end{array}$ & $\begin{array}{c}\text { Cr-ppi } \\
5\end{array}$ & $\begin{array}{c}\text { Cu-pppi } \\
s\end{array}$ & $\begin{array}{l}\text { La-ppi } \\
5\end{array}$ & $\begin{array}{c}\text { Mo-pp聐 } \\
5\end{array}$ & $\begin{array}{c}\mathrm{Nb}-\mathrm{ppm} \\
\mathrm{s}\end{array}$ \\
\hline 422002 & 50 & 1,500 & $\langle 1.0$ & N & 15 & 200 & 20 & 30 & N & $<20$ \\
\hline 422003 & 100 & 1,500 & 1.0 & $\mathrm{~N}$ & 20 & 300 & 30 & 50 & 7 & 20 \\
\hline 422004 & 100 & 1,500 & $\{1.0$ & N & 20 & 300 & 50 & 70 & $N$ & $N$ \\
\hline 422005 & 50 & 1,000 & $N$ & $\mathrm{~N}$ & 15 & 500 & 20 & 50 & $N$ & N \\
\hline 422006 & 70 & 2,000 & $\langle 1.0$ & $\mathrm{N}$ & 20 & 200 & 20 & 50 & $N$ & $<20$ \\
\hline 422007 & 50 & 1,500 & $\mathrm{~N}$ & $N$ & 15 & 200 & 20 & $N$ & $\mathrm{~N}$ & $<20$ \\
\hline 422008 & 70 & 1,500 & $\langle 1.0$ & $\mathrm{N}$ & 20 & 200 & 30 & 50 & $N$ & $<20$ \\
\hline 422009 & 70 & 1,500 & $\langle 1.0$ & N & 15 & 200 & 20 & 50 & $N$ & $<20$ \\
\hline 422010 & 50 & 1,000 & $\langle 1.0$ & N & 15 & 200 & 15 & 30 & $N$ & $N$ \\
\hline 422011 & 70 & 1,500 & 1.0 & $N$ & 15 & 200 & 15 & 50 & N & $<20$ \\
\hline 422012 & 70 & 1,000 & $\langle 1.0$ & $\mathrm{H}$ & 15 & 200 & 20 & 30 & N & $N$ \\
\hline 422013 & 50 & 1,000 & $\langle 1.0$ & $\mathrm{N}$ & 15 & 200 & 20 & 50 & $N$ & $<20$ \\
\hline 422015 & $\langle 10$ & 500 & 1.5 & $\mathrm{H}$ & $\mathrm{N}$ & 10 & 20 & $\mathrm{~N}$ & N & $\mathrm{H}$ \\
\hline 422016 & 50 & 500 & 1.0 & $\mathrm{~N}$ & N & 100 & 10 & $\mathrm{~N}$ & $N$ & $N$ \\
\hline 422017 & 50 & 700 & 1.0 & N & 15 & 150 & 20 & 30 & N & N \\
\hline 422019 & 70 & 1,500 & 1.0 & $N$ & 20 & 200 & 70 & 30 & $N$ & $<20$ \\
\hline 422020 & 30 & 700 & 1.0 & N & $\mathrm{N}$ & 30 & 10 & $\mathrm{~N}$ & N & $N$ \\
\hline 422022 & 100 & 1,500 & $\langle 1.0$ & N & 15 & 200 & 30 & 30 & $N$ & $<20$ \\
\hline 422023 & 10 & 700 & 1.0 & N & 10 & 20 & 10 & $N$ & N & N \\
\hline 422024 & 50 & 1,000 & $\langle 1.0$ & $\mathrm{N}$ & 15 & 200 & 30 & 20 & $\mathrm{~N}$ & $\mathrm{~N}$ \\
\hline 422025 & 10 & 700 & 1.0 & N & 10 & 30 & 15 & N & $N$ & N \\
\hline 422026 & 100 & 1,500 & 1.5 & $N$ & 30 & 300 & 50 & 50 & 5 & $<20$ \\
\hline 422027 & 100 & 1,500 & $\langle 1.0$ & N & 15 & 100 & 15 & 30 & 7 & $<20$ \\
\hline 422028 & 100 & 1,000 & 1.0 & $\mathrm{~N}$ & 15 & 150 & 20 & 50 & $N$ & $<20$ \\
\hline 422029 & 100 & 1,000 & 1.0 & N & 20 & 200 & 20 & 50 & $N$ & $<20$ \\
\hline 422030 & 70 & 700 & $N$ & $N$ & 15 & 200 & 15 & 30 & $\mathrm{~N}$ & $N$ \\
\hline 422031 & 70 & 1,000 & 1.0 & $\mathrm{~N}$ & 20 & 200 & 50 & 50 & N & $<20$ \\
\hline 422032 & 70 & 700 & 1.0 & N & 15 & 150 & 15 & 50 & $N$ & $<20$ \\
\hline 422033 & 20 & 1,000 & 1.0 & $\mathrm{~N}$ & 20 & 50 & 50 & 20 & N & N \\
\hline 422034 & 100 & 2,000 & $\langle 1.0$ & $\mathrm{N}$ & 20 & 200 & 50 & 50 & $\mathrm{~N}$ & 20 \\
\hline 422035 & 50 & 700 & $\langle 1.0$ & $N$ & 15 & 200 & 30 & 30 & $N$ & N \\
\hline 422036 & 70 & 1,000 & $\langle 1.0$ & $\mathrm{N}$ & 15 & 200 & 15 & 50 & N & $N$ \\
\hline 422037 & 50 & 1,000 & 1.0 & N & 15 & 200 & 20 & 50 & $N$ & $<20$ \\
\hline 422038 & 50 & 1,500 & $\langle 1.0$ & $\mathrm{N}$ & 15 & 200 & 20 & 50 & N & $<20$ \\
\hline 422039 & 70 & 1,000 & 1.0 & N & 15 & 200 & 20 & 30 & N & $\langle 20$ \\
\hline 422040 & 70 & 1,000 & 1.0 & $N$ & 15 & 200 & 20 & 50 & $N$ & $<20$ \\
\hline 422041 & 100 & 1,000 & 1.0 & N & 15 & 200 & 30 & 50 & $N$ & $<20$ \\
\hline 422042 & 70 & 1,000 & $\langle 1.0$ & N & 10 & 200 & 20 & 50 & $H$ & $N$ \\
\hline 422043 & 50 & 1,500 & 11.0 & $\mathrm{~N}$ & 15 & 200 & 15 & 50 & N & $<20$ \\
\hline 422044 & 70 & 2,000 & 1.0 & N & 15 & 200 & 20 & 50 & N & $<20$ \\
\hline 422045 & 70 & 1,500 & 1.0 & $N$ & 15 & 150 & 20 & 50 & N & $<20$ \\
\hline 422047 & 70 & 1,000 & $N$ & N & 15 & 200 & 20 & 50 & N & $<20$ \\
\hline 422048 & 100 & 1,000 & $\langle 1.0$ & N & 15 & 200 & 50 & 30 & $<5$ & N \\
\hline 422049 & 70 & 1,000 & $\langle 1.0$ & $N$ & 15 & 200 & 30 & 30 & N & $\mathrm{N}$ \\
\hline 422050 & 100 & 1,000 & 1.0 & N & 10 & 200 & 20 & 50 & $<5$ & $<20$ \\
\hline
\end{tabular}


TAELE 2.--RESULTS OF SPECTRDGRAPHIC ANALYSES OF NURE STKEAM-SEDIMENT SAMPLES FROM THE LIVENGDDD

AND WESTERN $1 / 3$ DF THE CIRCLE QUADRANGLES, ALASKA--Continued

\begin{tabular}{|c|c|c|c|c|c|c|c|c|c|c|c|}
\hline Sample & $\begin{array}{c}\mathrm{Ni}-\mathrm{ppo} \\
5\end{array}$ & $\begin{array}{c}\mathrm{Pb}-\mathrm{ppm} \\
s\end{array}$ & $\begin{array}{c}5 c-p p \text { i } \\
5\end{array}$ & $\begin{array}{c}5 n-p p m \\
s\end{array}$ & $\begin{array}{c}5 r-p p \\
s\end{array}$ & $\begin{array}{c}V \text {-ppa } \\
s\end{array}$ & $\begin{array}{c}W-p p \operatorname{an} \\
5\end{array}$ & $\begin{array}{c}Y \text {-ppi } \\
5\end{array}$ & $\begin{array}{c}\text { ln-pp西 } \\
5\end{array}$ & $\begin{array}{c}Z r-p p o n \\
s\end{array}$ & $\begin{array}{c}\text { Th-ppo } \\
s\end{array}$ \\
\hline 422002 & 50 & 20 & 20 & $N$ & 300 & 150 & $N$ & 30 & $N$ & 300 & $N$ \\
\hline 422003 & 70 & 30 & 20 & $N$ & 200 & 300 & $N$ & 50 & $<200$ & 500 & $N$ \\
\hline 422004 & 50 & 20 & 20 & $N$ & 100 & 200 & $N$ & 30 & $N$ & 150 & $N$ \\
\hline 422005 & 50 & 15 & 15 & $N$ & 300 & 150 & $N$ & 30 & $N$ & 500 & N \\
\hline $42200 t$ & 30 & 20 & 20 & $N$ & 500 & 200 & $N$ & 50 & $N$ & 300 & $N$ \\
\hline 422007 & 20 & 15 & 20 & $N$ & $N$ & 300 & $N$ & 30 & $N$ & 500 & $N$ \\
\hline 422008 & 50 & 20 & 20 & $N$ & 300 & 150 & $N$ & 30 & N & 200 & $N$ \\
\hline 422009 & 30 & 20 & 20 & $N$ & 300 & 200 & $N$ & 30 & $N$ & 200 & $N$ \\
\hline 422010 & 20 & 20 & 15 & N & 200 & 100 & $N$ & 30 & $N$ & 500 & $N$ \\
\hline 422011 & 30 & 15 & 20 & $N$ & 300 & 150 & $N$ & 30 & $N$ & 300 & $N$ \\
\hline 422012 & 30 & 20 & 15 & $N$ & 200 & 150 & N & 30 & $N$ & 200 & $N$ \\
\hline 422013 & 30 & 20 & 20 & N & 200 & 150 & $N$ & 30 & $N$ & 200 & $N$ \\
\hline 422015 & 15 & $\langle 10$ & 7 & $N$ & $N$ & 50 & $N$ & 20 & $N$ & 70 & $N$ \\
\hline 422016 & 20 & 20 & 10 & $N$ & 100 & 100 & N & 20 & $N$ & 100 & $N$ \\
\hline 422017 & 30 & 20 & 15 & $N$ & 150 & 150 & $N$ & 20 & $N$ & 150 & $N$ \\
\hline 422019 & 50 & 30 & 20 & N & 200 & 200 & N & 30 & N & 200 & $N$ \\
\hline 422020 & 20 & 10 & 10 & $N$ & 150 & 100 & N & 15 & $N$ & 150 & $N$ \\
\hline 422022 & 30 & 20 & 30 & N & 200 & 200 & $N$ & 50 & $N$ & 200 & $N$ \\
\hline 422023 & 10 & 10 & 7 & $N$ & 150 & 100 & $N$ & 15 & $N$ & 100 & $N$ \\
\hline 422024 & 30 & 20 & 20 & $N$ & 200 & 150 & $N$ & 20 & $N$ & 200 & $N$ \\
\hline 422025 & 30 & $<10$ & 7 & $N$ & 100 & 100 & $N$ & 15 & $N$ & 150 & $N$ \\
\hline 422026 & 100 & 300 & 30 & $N$ & 150 & 300 & $N$ & 50 & $N$ & 300 & $N$ \\
\hline 422027 & 20 & 30 & 20 & $N$ & 300 & 150 & N & 30 & $N$ & 700 & $N$ \\
\hline 422028 & 20 & 30 & 15 & N & 150 & 100 & $N$ & 30 & H & 700 & $N$ \\
\hline 422029 & 30 & 20 & 30 & $N$ & 300 & 150 & N & 50 & $N$ & 500 & $N$ \\
\hline 422030 & 20 & 20 & 15 & N & 200 & 100 & $N$ & 20 & $N$ & 500 & $N$ \\
\hline 422031 & 30 & 30 & 20 & $N$ & 200 & 200 & $N$ & 50 & $N$ & 300 & $N$ \\
\hline 422032 & 20 & 100 & 15 & N & 150 & 150 & N & 30 & 200 & 500 & $N$ \\
\hline 422033 & 30 & 50 & $<5$ & $N$ & 300 & 100 & $N$ & 30 & $N$ & 200 & N \\
\hline 422034 & 50 & 20 & 20 & $N$ & 300 & 200 & $N$ & 50 & $N$ & 500 & N \\
\hline 422035 & 30 & 15 & 20 & $N$ & 150 & 150 & $N$ & 20 & $N$ & 300 & $N$ \\
\hline 422036 & 30 & 20 & 20 & N & 300 & 150 & $N$ & 30 & $N$ & 700 & $N$ \\
\hline 422037 & 30 & 20 & 20 & $N$ & 300 & 150 & $N$ & 30 & $N$ & 500 & $N$ \\
\hline 422038 & 30 & 20 & 20 & $N$ & 300 & 150 & $N$ & 30 & N & 500 & $N$ \\
\hline 422039 & 30 & 20 & 20 & $N$ & 300 & 150 & $N$ & 30 & $N$ & 700 & $N$ \\
\hline 422040 & 30 & 20 & 20 & N & 300 & 150 & $N$ & 50 & $N$ & 700 & N \\
\hline 422041 & 30 & 30 & 20 & $N$ & 200 & 150 & $N$ & 30 & $N$ & 500 & $N$ \\
\hline 422042 & 30 & 20 & 20 & N & 200 & 200 & $N$ & 50 & $N$ & 500 & N \\
\hline 422043 & 30 & 20 & 20 & $N$ & 200 & 150 & $N$ & 30 & $N$ & 700 & $N$ \\
\hline 422044 & 30 & 20 & 20 & $N$ & 300 & 150 & $N$ & 50 & $N$ & 500 & N \\
\hline 422045 & 50 & 20 & 15 & $N$ & 200 & 150 & $N$ & 30 & $N$ & 700 & $N$ \\
\hline 422047 & 30 & 20 & 20 & $N$ & 300 & 200 & $N$ & 30 & $N$ & 300 & $N$ \\
\hline 422048 & 50 & 20 & 20 & $N$ & 150 & 200 & $N$ & 30 & N & 200 & $N$ \\
\hline 422049 & 50 & 15 & 30 & N & 150 & 300 & N & 30 & $N$ & 300 & $N$ \\
\hline 422050 & 20 & 30 & 20 & $N$ & 200 & 100 & $N$ & 50 & $N$ & 700 & $N$ \\
\hline
\end{tabular}


TABLE 2,--RESULTS OF SPECTRDGRAPHIC ANALYSES OF NURE GTREAM-GEDIMENT SAMPLES FRDM THE LIVENGOOD

AND WESTERN $1 / 3$ OF THE CIRCLE QUADRANGLES, ALASKA--Continued

\begin{tabular}{|c|c|c|c|c|c|c|c|c|c|}
\hline Sample & Latitude & Longitude & $\begin{array}{c}\text { Fe-pct. } \\
5\end{array}$ & $\begin{array}{c}M g-p c t . \\
5\end{array}$ & $\begin{array}{c}\text { Ca-pct. } \\
5\end{array}$ & $\begin{array}{c}\text { Ti-pct. } \\
5\end{array}$ & $\begin{array}{c}\text { Mn-ppi } \\
5\end{array}$ & $\begin{array}{c}A g-p p \text { i } \\
5\end{array}$ & $\begin{array}{c}A s-p p \text { m } \\
5\end{array}$ \\
\hline 422051 & $35 \quad 24 \quad 15$ & 1475030 & 10.00 & 2.00 & 5.0 & 1.000 & 1,500 & $N$ & H \\
\hline 422052 & 652332 & 1474422 & 7.00 & 2.00 & 5.0 & $>1.000$ & 1,500 & $N$ & $N$ \\
\hline 422053 & 652335 & 1472437 & 10.00 & 2.00 & 1.5 & 1.000 & 1,000 & $N$ & $N$ \\
\hline 422054 & 652240 & $14725 \quad 4$ & 7.00 & 2.00 & 2.0 & 1.000 & 1,000 & $N$ & $N$ \\
\hline 422055 & $b 52251$ & $14734 \quad 32$ & 3.00 & 1.00 & .5 & 1.000 & 500 & $N$ & $N$ \\
\hline $42205 b$ & 352237 & 1474252 & 1.50 & .50 & .3 & .500 & 500 & $N$ & $N$ \\
\hline 422057 & 652518 & 1481636 & 2.00 & .50 & .3 & .500 & 500 & N & N \\
\hline 422058 & 352747 & $14820 \quad 6$ & 10.00 & .70 & .1 & .700 & 2,000 & $N$ & $N$ \\
\hline 422059 & 351242 & $14855 b$ & 5.00 & 1.50 & 2.0 & 1.000 & 1,000 & N & $N$ \\
\hline 422060 & 651329 & $148 \quad 747$ & 7.00 & 2.00 & 3.0 & 1.000 & 1,000 & $N$ & N \\
\hline 422061 & 451636 & 14871 & 5.00 & 2.00 & 3.0 & 1.000 & 1,000 & $N$ & $N$ \\
\hline 422062 & 651712 & $148 \quad 8 \quad 17$ & 2.00 & 1.50 & .7 & .500 & 700 & $N$ & $N$ \\
\hline 422063 & 452317 & $148 \quad 1458$ & 5.00 & 2.00 & .7 & .700 & 1,000 & 6.5 & $N$ \\
\hline 422064 & 652634 & 1481436 & 10.00 & 3.00 & 2.0 & 1.000 & 2,000 & $<.5$ & $N$ \\
\hline 422065 & 65297 & $14822 \quad 5$ & 15.00 & 3.00 & 2.0 & 1.000 & $>5,000$ & 6.5 & $\mathrm{~N}$ \\
\hline 422066 & 652822 & $14831 \quad 9$ & 7.00 & 1.50 & .5 & .700 & 2,000 & 1.5 & N \\
\hline 422037 & 652538 & 148243 & 5.00 & 1.50 & 2.0 & 1.000 & 700 & 6.5 & $N$ \\
\hline 422068 & 652415 & $148 \quad 28 \quad 18$ & 7.00 & 2.00 & 3.0 & 1.000 & 700 & $N$ & $N$ \\
\hline 422049 & b5 2044 & 14810 & 5.00 & 2.00 & 2.0 & .700 & 1,000 & N & $n$ \\
\hline 422071 & b5 1911 & $14757 \quad 7$ & 5.00 & 1.50 & 1.5 & .700 & 500 & $N$ & N \\
\hline 422072 & 651918 & 1472953 & 5.00 & 1.50 & 2.0 & 1.000 & 1,000 & N & $N$ \\
\hline 422073 & 352159 & $147 \quad 1855$ & 7.00 & 1.50 & 2.0 & 1.000 & 1,500 & H & $N$ \\
\hline 422074 & 651927 & 1471030 & 10.00 & 3.00 & 2.0 & 1.000 & 1,000 & N & $n$ \\
\hline 422075 & 651950 & 1471924 & 5.00 & 1.50 & 2.0 & 1.000 & 700 & $N$ & $N$ \\
\hline 422076 & $6512 \quad 1$ & 1474936 & 5.00 & 1.50 & 2.0 & .700 & 500 & N & $N$ \\
\hline 422077 & 351229 & 1474144 & 5.00 & 1.50 & 2.0 & 1.000 & 700 & $N$ & $N$ \\
\hline 422078 & $6512 \quad 24$ & $14737 \quad 8$ & 5.00 & 1.50 & 1.5 & 1.000 & 500 & N & $N$ \\
\hline 422079 & 651238 & 1472916 & 7.00 & 1.50 & 3.0 & 1.000 & 700 & N & $N$ \\
\hline 422081 & 651153 & 1472451 & 7.00 & 1.50 & 1.5 & .700 & 500 & $<.5$ & $N$ \\
\hline 422082 & $6513 \quad 4$ & 147143 & 5.00 & 1.50 & 2.0 & 1.000 & 500 & $N$ & $N$ \\
\hline 422083 & $65 \quad 1049$ & 1471322 & 3.00 & 1.50 & 2.0 & .700 & 500 & $N$ & N \\
\hline 422084 & $65 \quad 15 \quad 42$ & $147103 b$ & 10.00 & 2.00 & 3.0 & 31.000 & 3,000 & $N$ & $N$ \\
\hline 422085 & 651448 & 1471855 & 7.00 & 1.50 & 1.5 & 1.000 & 700 & N & $N$ \\
\hline $42208 b$ & 651736 & 147165 & 10.00 & 2.00 & 3.0 & 1.000 & 1,000 & N & $N$ \\
\hline 422097 & 651742 & $147 \quad 1447$ & 7.00 & 1.50 & 2.0 & 1.000 & 700 & N & $n$ \\
\hline 422088 & 651748 & 14727 s & 5.00 & 1.50 & 1.5 & 31.000 & 1,500 & N & $N$ \\
\hline 422089 & 651520 & 1473154 & 10.00 & 2.00 & 2.0 & 1.000 & 1,000 & N & $n$ \\
\hline 422090 & $65 \quad 1458$ & $14733 \quad 6$ & 10.00 & 2.00 & 2.0 & 71.000 & 1,000 & N & $N$ \\
\hline 422091 & 65276 & 148425 & 7.00 & 2.00 & 1.5 & 1.000 & 1,000 & N & $N$ \\
\hline 422092 & 652635 & $148 \quad 48 \quad 2$ & 7.00 & 2.00 & 3.0 & .700 & 1,000 & $N$ & $N$ \\
\hline 422093 & 652526 & $14854 \quad 5$ & 7.00 & 2.00 & 1.5 & 1.000 & 700 & $N$ & N \\
\hline 422094 & b5 2222 & $14857 \quad$ & 5.00 & 1.50 & .5 & 31.000 & 700 & $N$ & $N$ \\
\hline 422095 & 651726 & $149 \quad 1543$ & 5.00 & 2.00 & 1.0 & 1.000 & 1,000 & $N$ & $N$ \\
\hline $42209 \mathrm{~s}$ & 651626 & 1492058 & 10.00 & 1.50 & 2.0 & 1.000 & 1,000 & $N$ & $N$ \\
\hline 422097 & 651451 & 1492738 & 10.00 & 1.50 & 2.0 & 1.000 & 700 & $N$ & $H$ \\
\hline
\end{tabular}


TABLE 2.--RESULTS OF SPECTROGRAPHIC ANALYSES OF NURE STREAM-SEDIMENT SAMPLES FROM THE LIVENGOOD

AND HESTERN $1 / 3$ OF THE CIRCLE QUADRANGLES, ALASKA--Continued

\begin{tabular}{|c|c|c|c|c|c|c|c|c|c|c|}
\hline Sample & $\begin{array}{c}\text { B-ppi } \\
5\end{array}$ & $\begin{array}{c}\text { Ba-ppri } \\
5\end{array}$ & $\begin{array}{c}\text { Be-ppm } \\
5\end{array}$ & 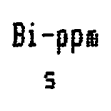 & $\begin{array}{c}\text { [o-ppi } \\
5\end{array}$ & $\begin{array}{c}C r-p p \text { 西 } \\
5\end{array}$ & $\begin{array}{c}\text { Cu-ppit } \\
5\end{array}$ & $\begin{array}{c}\mathrm{La}-p \rho \mathrm{I} \\
\mathrm{s}\end{array}$ & $\begin{array}{c}\text { Mo-ppm } \\
5\end{array}$ & 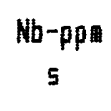 \\
\hline 422051 & 70 & 2,000 & 1.0 & $N$ & 20 & 300 & 50 & 30 & N & 20 \\
\hline 422052 & 70 & 2,000 & 11.0 & $N$ & 20 & 200 & 30 & 70 & $N$ & 20 \\
\hline 422053 & 100 & 700 & $\langle 1.0$ & $N$ & 20 & 150 & 70 & $N$ & $N$ & $N$ \\
\hline 422054 & 70 & 1,000 & $\langle 1.0$ & $N$ & 15 & 150 & 30 & 50 & $N$ & $<20$ \\
\hline 422055 & 50 & 500 & $N$ & $N$ & 10 & 150 & 15 & 30 & $\langle 5$ & $N$ \\
\hline 422056 & 70 & 300 & 1.0 & $N$ & 5 & 30 & 10 & $N$ & $<5$ & $N$ \\
\hline 422057 & 70 & 500 & $\langle 1.0$ & $N$ & 7 & 50 & 15 & 30 & N & $N$ \\
\hline 422058 & 100 & 1,000 & 1.0 & $N$ & 20 & 150 & 50 & 100 & $N$ & $<20$ \\
\hline 422059 & 100 & 1,000 & 1.0 & $N$ & 15 & 100 & 20 & 50 & $<5$ & $<20$ \\
\hline 422060 & 100 & 1,500 & 1.0 & $N$ & 20 & 100 & 20 & 50 & N & $\langle 20$ \\
\hline 422061 & 100 & 1,000 & $\langle 1.0$ & $N$ & 15 & 150 & 15 & 70 & $N$ & $<20$ \\
\hline 422062 & 70 & 1,000 & 1.5 & $N$ & 10 & 30 & 10 & $N$ & $N$ & $N$ \\
\hline 422063 & 100 & 700 & $\langle 1.0$ & $N$ & 20 & 100 & 20 & 30 & $N$ & $N$ \\
\hline 422064 & 100 & 1,500 & 11.0 & $N$ & 20 & 150 & 70 & 30 & $N$ & $N$ \\
\hline 422065 & 70 & 2,000 & 1.0 & $N$ & 20 & 150 & 100 & $N$ & $\langle 5$ & $N$ \\
\hline 422066 & 70 & 1,000 & 41.0 & $\$ 10$ & 30 & 200 & 100 & 50 & 7 & $N$ \\
\hline 422067 & 70 & 1,500 & 1.0 & $N$ & 15 & 100 & 20 & 50 & $N$ & $<20$ \\
\hline 422068 & 100 & 1,500 & $\langle 1.0$ & $N$ & 15 & 200 & 20 & 50 & $N$ & $\langle 20$ \\
\hline 422069 & 70 & 1,000 & $\langle 1.0$ & $N$ & 20 & 150 & 20 & 50 & $N$ & $N$ \\
\hline 422071 & 70 & 1,000 & $\$ 1.0$ & N & 15 & 150 & 30 & 30 & $N$ & $N$ \\
\hline 422072 & 100 & 1,500 & 1.0 & $N$ & 15 & 150 & 20 & 50 & $N$ & $<20$ \\
\hline 422073 & 100 & 1,500 & 1.0 & $N$ & 20 & 150 & 20 & 50 & $N$ & $\langle 20$ \\
\hline 422074 & 50 & 1,500 & $N$ & $\mathbf{N}$ & 15 & 150 & 20 & 50 & $N$ & $N$ \\
\hline 422075 & 70 & 1,500 & 1.0 & $N$ & 15 & 150 & 15 & 50 & $H$ & 20 \\
\hline 422076 & 70 & 1,500 & 1.0 & $N$ & 15 & 100 & 15 & 50 & $N$ & $<20$ \\
\hline 422077 & 100 & 1,000 & $\langle 1.0$ & $N$ & 15 & 150 & 15 & 70 & $N$ & 120 \\
\hline 422078 & 70 & 1,000 & $\langle 1,0$ & $N$ & 20 & 150 & 20 & 50 & $N$ & $<20$ \\
\hline 422079 & 70 & 1,000 & $N$ & $N$ & 15 & 150 & 15 & 70 & N & 20 \\
\hline 422081 & 100 & 1,500 & 1.0 & $N$ & 20 & 150 & 30 & 70 & $N$ & 20 \\
\hline 422092 & 100 & 1,000 & 1.0 & $N$ & 15 & 150 & 15 & 70 & $N$ & $<20$ \\
\hline 422083 & 70 & 700 & $<1.0$ & $N$ & 15 & 150 & 15 & 50 & $N$ & $<20$ \\
\hline 422084 & 70 & 1,000 & 41.0 & $N$ & 15 & 300 & 15 & 70 & $N$ & $<20$ \\
\hline 422085 & 50 & 700 & $N$ & $N$ & 15 & 15 & 15 & 50 & $N$ & $N$ \\
\hline 422086 & 70 & 1,000 & 1.0 & $N$ & 20 & 150 & 20 & 50 & N & $<20$ \\
\hline 422087 & 50 & 700 & $\langle 1.0$ & $N$ & 10 & 10 & 10 & 30 & $N$ & $<20$ \\
\hline 422098 & 100 & 700 & 1.0 & $N$ & 15 & 70 & 15 & 30 & $N$ & $<20$ \\
\hline 422089 & 70 & 1,000 & 1.0 & $N$ & 20 & 150 & 30 & 50 & $N$ & $N$ \\
\hline 422090 & 50 & 700 & $N$ & $N$ & 15 & 150 & 30 & 20 & $N$ & $<20$ \\
\hline 422091 & 100 & 1,000 & 1.0 & $N$ & 20 & 150 & 30 & 50 & $N$ & $<20$ \\
\hline 422092 & 70 & 1,500 & 1.0 & $N$ & 15 & 150 & 15 & 50 & N & $N$ \\
\hline 422093 & 70 & 1,000 & 1.0 & $N$ & 30 & 150 & 30 & 70 & $N$ & N \\
\hline 422094 & 100 & 500 & 1.0 & $N$ & 15 & 150 & 15 & 50 & $N$ & 20 \\
\hline 422095 & 70 & 700 & 1.0 & $N$ & 20 & 150 & 20 & 50 & N & 20 \\
\hline 422096 & 70 & 1,000 & 1.0 & $N$ & 20 & 150 & 15 & 70 & $N$ & 20 \\
\hline 422097 & 100 & 1,000 & $<1.0$ & $N$ & 15 & 100 & 10 & 50 & $N$ & $N$ \\
\hline
\end{tabular}


TAELE 2. - RESULTS OF SPECTROGRAPHIC ANALYSES OF NURE STREAM-SEDIMENT SAMPLES FROH THE LIVENGOOD

AND WESTERN $1 / 3$ DF THE CIRCLE QUADRANGLES, ALASKA--ContinUEd

\begin{tabular}{|c|c|c|c|c|c|c|c|c|c|c|c|}
\hline Sample & $\begin{array}{c}\mathrm{Ni}-\mathrm{pp} \\
\mathrm{s}\end{array}$ & $\begin{array}{c}\mathrm{Pb}-\mathrm{ppa} \\
5\end{array}$ & $\begin{array}{c}\text { St-ppa } \\
s\end{array}$ & $\begin{array}{c}\text { Sn-pp } \\
5\end{array}$ & $\begin{array}{c}\text { Sr-ppit } \\
5\end{array}$ & $\begin{array}{c}V \text {-ppi } \\
5\end{array}$ & $\begin{array}{c}W-p p i \text { il } \\
5\end{array}$ & $\begin{array}{c}y-p p \text { i } \\
5\end{array}$ & $\begin{array}{c}\text { Zn-ppi } \\
5\end{array}$ & $\begin{array}{c}2 r-p \rho \\
s\end{array}$ & $\begin{array}{c}\text { Th-ppa } \\
s\end{array}$ \\
\hline 422051 & 50 & 30 & 20 & $N$ & 300 & 200 & $H$ & 30 & $N$ & 300 & $N$ \\
\hline 422052 & 50 & 20 & 20 & $N$ & 300 & 200 & $N$ & 50 & $N$ & 500 & $N$ \\
\hline 422053 & 50 & 30 & 20 & N & 100 & 200 & $N$ & 30 & $N$ & 300 & $H$ \\
\hline 422054 & 30 & 20 & 20 & $N$ & 200 & 200 & $N$ & 50 & $N$ & 500 & $N$ \\
\hline 422055 & 20 & 15 & 10 & $N$ & 100 & 100 & $N$ & 20 & $N$ & 700 & $N$ \\
\hline 422056 & 10 & 15 & 5 & $N$ & $<100$ & 50 & $N$ & 10 & $N$ & 200 & $N$ \\
\hline 422057 & 20 & 15 & 7 & $N$ & 100 & 70 & $N$ & 20 & $N$ & 200 & $n$ \\
\hline 422058 & 50 & 50 & 20 & H & 200 & 100 & N & 30 & $N$ & 500 & $N$ \\
\hline 422059 & 50 & 30 & 15 & $N$ & 200 & 150 & N & 50 & $N$ & 500 & $N$ \\
\hline 422060 & 50 & 20 & 20 & $N$ & 200 & 150 & $N$ & 50 & $N$ & 300 & $\mathrm{H}$ \\
\hline 422061 & 50 & 20 & 20 & $N$ & 200 & 100 & $N$ & 50 & $N$ & 500 & $N$ \\
\hline 422062 & 30 & 10 & 7 & $N$ & $<100$ & 100 & $N$ & 10 & $N$ & 150 & $N$ \\
\hline 422063 & 50 & 20 & 15 & $N$ & 100 & 150 & $N$ & 30 & $N$ & 150 & $N$ \\
\hline 422064 & 50 & 30 & 20 & $N$ & 200 & 150 & N & 50 & $N$ & 150 & $N$ \\
\hline 422065 & 50 & 20 & 30 & N & N & 200 & N & 50 & $N$ & 200 & $N$ \\
\hline 422066 & 50 & 100 & 20 & $N$ & 150 & 150 & $N$ & 50 & 300 & 150 & $N$ \\
\hline 422067 & 70 & 50 & 20 & N & 300 & 150 & $N$ & 50 & $N$ & 200 & $N$ \\
\hline 422068 & 50 & 20 & 20 & $N$ & 300 & 150 & $N$ & 50 & $N$ & 300 & $N$ \\
\hline 422069 & 50 & 20 & 20 & $N$ & 200 & 150 & $N$ & 30 & $H$ & 200 & $N$ \\
\hline 422071 & 50 & 30 & 20 & $N$ & 150 & 150 & $N$ & 30 & $N$ & 150 & $N$ \\
\hline 422072 & 50 & 20 & 30 & $N$ & 100 & 150 & N & 50 & $N$ & 300 & $N$ \\
\hline 422073 & 50 & 30 & 30 & $N$ & 200 & 150 & N & 50 & $N$ & 300 & $N$ \\
\hline 422074 & 50 & 30 & 20 & $N$ & 300 & 300 & N & 30 & $N$ & 200 & $N$ \\
\hline 422075 & 30 & 20 & 20 & $N$ & 200 & 100 & $N$ & 50 & $N$ & 200 & $N$ \\
\hline 422076 & 50 & 20 & 20 & $N$ & 200 & 150 & $N$ & 50 & $N$ & 200 & $N$ \\
\hline 422077 & 30 & 30 & 20 & $N$ & 200 & 150 & $N$ & 50 & $N$ & 200 & $N$ \\
\hline 422078 & 30 & 20 & 20 & $N$ & 150 & 150 & $N$ & 50 & $N$ & 200 & $N$ \\
\hline 422079 & 30 & 20 & 15 & $N$ & 300 & 200 & $N$ & 50 & $N$ & 700 & N \\
\hline 422081 & 50 & 50 & 30 & $N$ & 200 & 150 & $N$ & 50 & $N$ & 150 & $N$ \\
\hline 422082 & 30 & 30 & 20 & $N$ & 200 & 150 & $N$ & 50 & $n$ & 200 & N \\
\hline 422083 & 30 & 20 & 20 & $N$ & 200 & 100 & $N$ & 30 & $N$ & 200 & $N$ \\
\hline 422084 & 50 & 20 & 20 & $N$ & 300 & 150 & $N$ & 50 & $N$ & 31,000 & $N$ \\
\hline 422085 & 30 & 20 & 15 & $N$ & 100 & 100 & $N$ & 20 & $N$ & 700 & $N$ \\
\hline 422086 & 50 & 20 & 20 & $N$ & 200 & 150 & $n$ & 50 & $N$ & 500 & $N$ \\
\hline 422087 & 30 & 10 & 10 & $N$ & $<100$ & 100 & $N$ & 30 & $N$ & 700 & $N$ \\
\hline 422088 & 30 & 30 & 15 & $N$ & 150 & 100 & $N$ & 30 & $N$ & 300 & $N$ \\
\hline 422089 & 50 & 30 & 20 & $N$ & 200 & 150 & $N$ & 50 & $N$ & 300 & $N$ \\
\hline 422090 & 50 & 20 & 20 & $N$ & 300 & 150 & $N$ & 30 & $N$ & 200 & $N$ \\
\hline 422091 & 50 & 30 & 20 & N & 200 & 150 & $N$ & 30 & $N$ & 500 & $N$ \\
\hline 422092 & 30 & 30 & 20 & $N$ & 300 & 100 & $N$ & 50 & $N$ & 300 & $N$ \\
\hline 422093 & 50 & 30 & 20 & $N$ & 150 & 150 & N & 50 & $N$ & 200 & $N$ \\
\hline 422094 & 30 & 30 & 10 & $N$ & 100 & 70 & $N$ & 30 & $N$ & 300 & $N$ \\
\hline 422095 & 50 & 30 & 15 & $\mathrm{~N}$ & 150 & 100 & N & 30 & $N$ & 300 & $N$ \\
\hline 422096 & 30 & 30 & 20 & $N$ & 200 & 100 & $N$ & 50 & $N$ & 300 & $N$ \\
\hline 422097 & 20 & 30 & 15 & $N$ & 200 & 150 & N & 30 & $N$ & 300 & $n$ \\
\hline
\end{tabular}


TABLE 2.--RESUL TS OF SPECTROGRAPHIC AMALYSES OF NURE STREAM-GEDIMENT SAMPLES FROM THE LIVENGOOD AND WESTERN $1 / 3$ OF THE CIRCLE QUADRANGLES, ALASKA--ContinUed

\begin{tabular}{|c|c|c|c|c|c|c|c|c|c|}
\hline Sa䨆le & Latitude & Longitude & $\begin{array}{c}\text { Fe-pct. } \\
5\end{array}$ & $\underset{s}{M_{g}-p c t .}$ & $\begin{array}{c}\text { Ca-pct. } \\
s\end{array}$ & $\begin{array}{c}\text { Ti-pct. } \\
5\end{array}$ & $\begin{array}{c}M_{n}-p p \text { M } \\
5\end{array}$ & $\begin{array}{c}\mathrm{Ag}-\mathrm{ppm} \\
5\end{array}$ & $\begin{array}{c}\text { As-pp啇 } \\
5\end{array}$ \\
\hline 422098 & 651359 & 1493221 & 10.00 & 2.00 & 3.0 & 1.000 & 1,500 & N & $N$ \\
\hline 422099 & 65135 & 1493822 & 10.00 & 2.00 & 3.0 & 1.000 & 1,000 & N & N \\
\hline 422100 & $6512 \quad 2$ & 1494936 & 7.00 & 2.00 & 3.0 & 1.000 & 700 & N & N \\
\hline 422101 & 651049 & 1495541 & 7.00 & 2.00 & 3.0 & 1.000 & 700 & $\mathrm{~N}$ & $\ddot{N}$ \\
\hline 422102 & 65929 & 149245 & 10.00 & 5.00 & 1.5 & .700 & 3,000 & N & $N$ \\
\hline 422103 & 651654 & $147 \quad 3852$ & 7.00 & 5.00 & 1.0 & .700 & 500 & N & N \\
\hline$\$ 22104$ & $6516 \quad 14$ & 147413 & 10.00 & 2.00 & 2.0 & 1.000 & 2,000 & N & $N$ \\
\hline 422105 & 651941 & 1482958 & 7.00 & 2.00 & .7 & .700 & 700 & $N$ & N \\
\hline 422106 & 651838 & $14823 \quad 13$ & 7.00 & 2.00 & .5 & 1.000 & 1,000 & N & N \\
\hline 422107 & 651653 & 1481046 & 10.00 & 1.50 & 2.0 & 1.000 & 2,000 & $<.5$ & $N$ \\
\hline 422108 & 651436 & 1481014 & 10.00 & 2.00 & .5 & 1.000 & 1,000 & N & $N$ \\
\hline 422109 & 651059 & $148 \quad 1057$ & 10.00 & 1.50 & 2.0 & 1.000 & 1,000 & N & N \\
\hline$\$ 22110$ & 65854 & $148 \quad 1453$ & 10.00 & 3.00 & 2.0 & 1.000 & 1,500 & .5 & $N$ \\
\hline 422111 & 65749 & 148511 & 10.00 & 2.00 & 3.0 & 1.000 & 1,000 & $<.5$ & N \\
\hline 422112 & 65959 & $14749 \quad 5$ & 10.00 & 2.00 & 3.0 & 1.000 & 1,000 & $N$ & $N$ \\
\hline 422113 & 65925 & 1474730 & 7.00 & 2.00 & 3.0 & 1.000 & 1,000 & N & $N$ \\
\hline 422114 & 65939 & 1473619 & 10.00 & 2.00 & 2.0 & 1.000 & 1,500 & $N$ & N \\
\hline 422115 & 65916 & 1473714 & 10.00 & 2.00 & 5.0 & 1.000 & 1,500 & $N$ & $N$ \\
\hline 422116 & $65 \quad 925$ & 1471551 & 7.00 & 2.00 & 2.0 & 1.000 & 1,000 & $<.5$ & $N$ \\
\hline 422118 & 6590 & 1471239 & 7.00 & 2.00 & 5.0 & 1.000 & 1,000 & $N$ & $N$ \\
\hline 422119 & $65 \quad 812$ & $147 \quad 6 \quad 47$ & 10.00 & 2.00 & 3.0 & .700 & 1,000 & $N$ & N \\
\hline 422120 & $65 \quad 619$ & $14735 \quad 6$ & 7.00 & 2,00 & 3.0 & 71.000 & 700 & N & N \\
\hline 422121 & $65 \quad 621$ & 1471758 & 7.00 & 1.50 & 3.0 & 1.000 & 1,000 & N & N \\
\hline 422122 & $65 \quad 6 \quad 46$ & $147 \quad 17 \quad 9$ & 15.00 & 2.00 & 3.0 & 31.000 & 3,000 & N & N \\
\hline 422123 & $65 \quad 657$ & $147 \quad 8 \quad 2$ & 7.00 & 2.00 & 3.0 & 1.000 & 1,000 & $N$ & $N$ \\
\hline 422124 & $65 \quad 549$ & 14728 & 15.00 & 3.00 & 5.0 & 1.000 & 2,000 & N & $N$ \\
\hline 422125 & 65140 & 14758 & 7.00 & 2.00 & 3.0 & 1.000 & 700 & $N$ & $\ddot{N}$ \\
\hline 422126 & 65413 & $14710 \quad 11$ & 10.00 & 2.00 & 3.0 & 1.000 & 2,000 & N & $N$ \\
\hline 422127 & 6518 & 1472344 & 10.00 & 2.00 & 3.0 & 1.000 & 2,000 & N & $N$ \\
\hline 422128 & 65318 & 1473518 & 10.00 & 2.00 & 3.0 & 31.000 & 1,500 & N & $N$ \\
\hline 422130 & $6528 \quad 4$ & $148 \quad 2439$ & 7.00 & 2.00 & 3.0 & 1.000 & 1,500 & $N$ & $N$ \\
\hline 422131 & 652254 & $148 \quad 12 \quad 10$ & 10.00 & 2.00 & 2.0 & 1.000 & 1,500 & $\mathrm{~N}$ & N \\
\hline 422132 & 651939 & 1474656 & 15.00 & 2.00 & 2.0 & 1.000 & 1,500 & 1.0 & $\ddot{N}$ \\
\hline 422133 & 652022 & 1474420 & 15.00 & 2.00 & 3.0 & 1.000 & 2,000 & $N$ & $\ddot{N}$ \\
\hline 422134 & 652030 & 147345 & 10.00 & 1.50 & 2.0 & .700 & 500 & N & N \\
\hline 422135 & 652017 & 1472430 & 15.00 & 1.50 & .5 & $>1.000$ & 2,000 & N & $N$ \\
\hline 422136 & 652355 & $147 \quad 1221$ & 10.00 & 2.00 & .7 & $>1.000$ & 2,000 & N & $N$ \\
\hline 422137 & 652223 & 1471127 & 15.00 & 2.00 & 5.0 & 31.000 & 700 & N & $\mathrm{N}$ \\
\hline 422138 & 651028 & 1471753 & 15.00 & 2.00 & 5.0 & $>1.000$ & 1,000 & N & $N$ \\
\hline 422139 & 651251 & 147109 & 15.00 & 2.00 & 5.0 & 1.000 & 1,500 & N & $N$ \\
\hline 422140 & 65213 & $149 \quad 032$ & 10.00 & 2.00 & 5.0 & 1.000 & 2,000 & $N$ & N \\
\hline 422141 & 651628 & 1474716 & 2.00 & .70 & 1.0 & .200 & 500 & N & N \\
\hline 422142 & 65158 & 1474957 & 20.00 & 2.00 & 5.0 & 1.000 & 2,000 & $\ddot{N}$ & $\ddot{N}$ \\
\hline 422143 & 651933 & $148 \quad 1945$ & 15.00 & 2.00 & 5.0 & 31.000 & 2,000 & N & H \\
\hline 422144 & $65 \quad 1619$ & $148 \quad 2013$ & 15.00 & 2.00 & 5.0 & $>1.000$ & 2,000 & N & N \\
\hline
\end{tabular}


TAELE 2.--RESULTS OF SPECTROGRAPHIC ANALYSES OF NURE STREAM-SEDIMENT SAMPLES FROM THE LIVENGOOD AND WESTERN $1 / 3$ OF THE CIRCLE QUADRANGLES, ALASKA--Conti nued

\begin{tabular}{|c|c|c|c|c|c|c|c|c|c|c|}
\hline Sa㽢le & $\begin{array}{c}\text { B-ppin } \\
5\end{array}$ & $\begin{array}{c}\text { Ba-ppm } \\
5\end{array}$ & $\begin{array}{c}\text { Be-ppi } \\
5\end{array}$ & $\underset{5}{\mathrm{Bi}-\mathrm{ppD}}$ & $\begin{array}{c}\text { Co-ppin } \\
5\end{array}$ & $\begin{array}{c}\text { Cr-ppi } \\
5\end{array}$ & $\begin{array}{c}\text { Cu-ppp } \\
5\end{array}$ & $\begin{array}{c}\text { La-pp } \\
5\end{array}$ & $\begin{array}{c}\text { Mo-ppi } \\
5\end{array}$ & $\begin{array}{c}\mathrm{Nb}-p p \mathrm{~m} \\
5\end{array}$ \\
\hline 422098 & 70 & 1,500 & $\langle 1.0$ & N & 20 & 150 & 15 & 50 & N & $<20$ \\
\hline 422099 & 70 & 1,500 & $\{1.0$ & $N$ & 15 & 200 & 20 & 50 & $N$ & 20 \\
\hline 422100 & 70 & 1,500 & 1.0 & N & 20 & 150 & 15 & 50 & N & $N$ \\
\hline 422101 & 70 & 1,500 & 11.0 & N & 15 & 200 & 20 & 50 & N & $<20$ \\
\hline 422102 & 100 & 1,000 & 1.0 & N & 20 & 500 & 30 & $N$ & $\langle 5$ & $N$ \\
\hline 422103 & 50 & 700 & N & N & 15 & 300 & 20 & 30 & N & $N$ \\
\hline 422104 & 70 & 1,500 & $\langle 1.0$ & N & 20 & 300 & 30 & 50 & $<5$ & 20 \\
\hline 422105 & 100 & 1,500 & 1.0 & N & 15 & 150 & 50 & 50 & $<5$ & $<20$ \\
\hline 422106 & 50 & 700 & $N$ & $N$ & 20 & 200 & 50 & 30 & $N$ & $N$ \\
\hline 422107 & 200 & 1,500 & $N$ & N & 20 & 200 & 70 & 70 & 10 & $N$ \\
\hline 422108 & 200 & 1,500 & 1.0 & $\mathrm{~N}$ & 15 & 200 & 20 & 50 & $<5$ & 20 \\
\hline 422109 & 100 & 2,000 & $N$ & N & 15 & 150 & 50 & 30 & N & $N$ \\
\hline 422110 & 50 & 2,000 & N & $\mathrm{N}$ & 20 & 300 & 70 & N & $N$ & $N$ \\
\hline 422111 & 70 & 2,000 & 41.0 & N & 20 & 200 & 30 & 50 & $N$ & N \\
\hline 422112 & 70 & 1,500 & N & $N$ & 20 & 150 & 30 & 50 & $N$ & $<20$ \\
\hline 422113 & 70 & 2,000 & $\langle 1.0$ & $N$ & 15 & 150 & 30 & 50 & $N$ & $<20$ \\
\hline 422114 & 70 & 2,000 & N & $\mathrm{N}$ & 20 & 200 & 30 & 30 & $N$ & N \\
\hline 422115 & 100 & 2,000 & 1.0 & N & 20 & 200 & 30 & 50 & $N$ & $<20$ \\
\hline 422116 & 70 & 1,500 & 1.0 & N & 20 & 200 & 30 & $N$ & $N$ & $N$ \\
\hline 422118 & 50 & 1,500 & N & $\mathrm{N}$ & 15 & 150 & 20 & 50 & N & $<20$ \\
\hline 422119 & 70 & 1,500 & $N$ & $N$ & 15 & 150 & 20 & N & $N$ & N \\
\hline 422120 & 50 & 1,500 & $\langle 1.0$ & $N$ & 15 & 150 & 20 & 50 & $N$ & N \\
\hline 422121 & 30 & 1,500 & N & $\mathrm{N}$ & 15 & 100 & 20 & 30 & N & $N$ \\
\hline 422122 & 100 & 2,000 & 1.0 & $\mathrm{~N}$ & 30 & 100 & 50 & 50 & N & $<20$ \\
\hline 422123 & 50 & 1,500 & 1.0 & N & 20 & 100 & 30 & 50 & $N$ & N \\
\hline 422124 & 50 & 2,000 & 1.0 & N & 20 & 200 & 70 & 50 & N & N \\
\hline 422125 & 70 & 2,000 & $\langle 1.0$ & N & 15 & 150 & 30 & 50 & $N$ & $N$ \\
\hline 422126 & 100 & 2,000 & 1.0 & $N$ & 20 & 200 & 30 & 70 & $N$ & $<20$ \\
\hline 422127 & 70 & 1,500 & $\langle 1.0$ & $N$ & 30 & 150 & 50 & 50 & $N$ & $<20$ \\
\hline 422128 & 70 & 1,000 & N & N & 20 & 200 & 20 & 70 & $N$ & $<20$ \\
\hline 422130 & 70 & 1,000 & N & $N$ & 20 & 200 & 30 & 50 & $N$ & $N$ \\
\hline 422131 & 100 & 1,000 & 1.0 & N & 20 & 200 & 30 & 70 & $N$ & 20 \\
\hline 422132 & 30 & 700 & $\mathrm{~N}$ & N & 15 & 150 & 20 & 50 & N & $N$ \\
\hline 422133 & 50 & 1,000 & $\{1.0$ & N & 15 & 150 & 15 & 50 & $N$ & $N$ \\
\hline 422134 & 50 & 1,000 & $\langle 1.0$ & N & 15 & 150 & 20 & 50 & N & N \\
\hline 422135 & 100 & 700 & $\langle 1.0$ & $N$ & 15 & 70 & 50 & N & $N$ & $<20$ \\
\hline 422136 & 100 & 700 & 1.0 & N & 20 & 100 & 30 & 30 & $N$ & 20 \\
\hline 422137 & 100 & 2,000 & 1.5 & N & 15 & 200 & 50 & 70 & $N$ & 20 \\
\hline 422138 & 70 & 1,500 & 1.5 & N & 15 & 150 & 30 & 70 & $N$ & $<20$ \\
\hline 422139 & 100 & 1,500 & 1.0 & $N$ & 20 & 200 & 50 & 50 & $<5$ & $<20$ \\
\hline 422140 & 150 & 1,000 & 1.0 & N & 15 & 200 & 20 & 50 & $N$ & $<20$ \\
\hline 422141 & $<10$ & 700 & 1.0 & N & $N$ & 20 & 10 & N & N & N \\
\hline 422142 & 100 & 1,500 & $\langle 1.0$ & N & 20 & 200 & 50 & 50 & $\langle 5$ & $<20$ \\
\hline 422143 & 150 & 1,000 & $\langle 1.0$ & $N$ & 15 & 200 & 50 & 50 & $\mathrm{~N}$ & 30 \\
\hline 422144 & 150 & 1,500 & 1.0 & $\mathrm{~N}$ & 15 & 200 & 30 & 50 & N & 20 \\
\hline
\end{tabular}


TABLE 2.--RESULTS OF SPECTROGRAPHIC ANALYSES OF NURE STREAH-SEDIMENT SAMPLES FROM THE LIVENGOOD

AND WESTERN $1 / 3$ OF THE CIRCLE QUADRANGLES, ALASKA--ContinuEd

\begin{tabular}{|c|c|c|c|c|c|c|c|c|c|c|c|}
\hline Sample & $\begin{array}{c}N i-p p i \\
5\end{array}$ & $\begin{array}{c}P b-p p m \\
5\end{array}$ & $\begin{array}{c}\text { Sc-pp } \\
5\end{array}$ & $\begin{array}{c}5 n-p p \text { i } \\
5\end{array}$ & $\begin{array}{c}S r-p p \text { a } \\
5\end{array}$ & $\begin{array}{c}V \text {-ppi } \\
5\end{array}$ & $\begin{array}{c}H-p p \\
5\end{array}$ & $\begin{array}{c}\text { Y-pp } \\
5\end{array}$ & $\begin{array}{c}2 n-p p a \\
5\end{array}$ & $\begin{array}{c}\mathrm{Ir}-\mathrm{pp} \\
\mathrm{s}\end{array}$ & $\begin{array}{c}\text { Th-ppa } \\
5\end{array}$ \\
\hline 422098 & 30 & 30 & 15 & $N$ & 300 & 200 & $N$ & 50 & $N$ & 300 & $N$ \\
\hline 422099 & 30 & 30 & 20 & $N$ & 300 & 200 & $N$ & 50 & $N$ & 300 & $N$ \\
\hline 422100 & 30 & 30 & 20 & $N$ & 300 & 150 & $N$ & 50 & $N$ & 300 & $N$ \\
\hline 422101 & 70 & 20 & 20 & $N$ & 300 & 300 & $N$ & 50 & $\mathbb{N}$ & 300 & $N$ \\
\hline 422102 & 200 & 20 & 20 & $N$ & 150 & 200 & $N$ & 30 & $N$ & 150 & $N$ \\
\hline 422103 & 150 & 20 & 15 & $N$ & 200 & 200 & $N$ & 20 & $N$ & 150 & $N$ \\
\hline 422104 & 70 & 20 & 20 & $N$ & 200 & 300 & $N$ & 50 & $N$ & 300 & $N$ \\
\hline 422105 & 70 & 30 & 20 & $N$ & 150 & 300 & $N$ & 30 & $N$ & 200 & $N$ \\
\hline 422106 & 50 & 20 & 20 & $N$ & $\langle 100$ & 200 & $N$ & 30 & $N$ & 150 & $N$ \\
\hline 422107 & 50 & 30 & 20 & $N$ & 200 & 500 & N & 50 & $N$ & 300 & $N$ \\
\hline $42210 \mathrm{~B}$ & 50 & 30 & 20 & $N$ & 100 & 300 & $N$ & 50 & 200 & 300 & $N$ \\
\hline 422109 & 50 & 10 & 20 & $N$ & 200 & 200 & $N$ & 30 & $N$ & 300 & $N$ \\
\hline 422110 & 50 & 30 & 20 & $N$ & $<100$ & 500 & N & 30 & $N$ & 200 & $N$ \\
\hline 422111 & 50 & 20 & 20 & $N$ & 200 & 500 & $N$ & 30 & $N$ & 500 & $N$ \\
\hline 422112 & 30 & 20 & 20 & $N$ & 200 & 200 & $N$ & 50 & $N$ & 500 & $N$ \\
\hline 422113 & 50 & 20 & 15 & $N$ & 300 & 200 & $N$ & 50 & $N$ & 500 & $N$ \\
\hline 422114 & 50 & 20 & 20 & $N$ & $<100$ & 500 & $N$ & 30 & $N$ & 500 & $N$ \\
\hline 422115 & 50 & 30 & 20 & $N$ & 300 & 300 & N & 50 & $<200$ & 500 & $N$ \\
\hline 422116 & 50 & 30 & 15 & $N$ & 200 & 200 & $N$ & 30 & $N$ & 300 & $N$ \\
\hline 422118 & 20 & 20 & 20 & $N$ & 300 & 200 & N & 50 & $N$ & 500 & $N$ \\
\hline 422119 & 30 & 20 & 15 & $N$ & 200 & 300 & $N$ & 30 & $N$ & 500 & $N$ \\
\hline 422120 & 20 & 20 & 20 & $N$ & 200 & 300 & $N$ & 50 & $N$ & 500 & $N$ \\
\hline 422121 & 20 & 20 & 20 & $N$ & 200 & 300 & $N$ & 30 & $N$ & 300 & $N$ \\
\hline 422122 & 50 & 50 & 20 & $N$ & 200 & 500 & $N$ & 50 & $\mathbf{N}$ & 300 & $N$ \\
\hline 422123 & 30 & 30 & 20 & $N$ & 200 & 300 & $N$ & 50 & $N$ & 500 & $N$ \\
\hline 422124 & 30 & 20 & 20 & $N$ & $\measuredangle 100$ & 500 & $N$ & 50 & $N$ & 500 & $N$ \\
\hline 422125 & 30 & 20 & 20 & $N$ & 200 & 300 & $N$ & 50 & $N$ & 500 & $N$ \\
\hline $42212 b$ & 50 & 20 & 15 & $N$ & 200 & 300 & $N$ & 50 & $N$ & 700 & $N$ \\
\hline 422127 & 50 & 30 & 20 & $N$ & 200 & 200 & $N$ & 50 & $N$ & 300 & $N$ \\
\hline 422128 & 30 & 20 & 15 & $N$ & 200 & 200 & $N$ & 70 & $N$ & $>1,000$ & $N$ \\
\hline 422130 & 30 & 20 & 15 & N & 200 & 150 & $N$ & 50 & $N$ & 1,000 & $N$ \\
\hline 422131 & 50 & 30 & 15 & N & 200 & 150 & $N$ & 50 & $N$ & 500 & $N$ \\
\hline 422132 & 20 & 20 & 10 & $N$ & 200 & 300 & $N$ & 20 & $N$ & 700 & $N$ \\
\hline 422133 & 20 & 20 & 20 & $N$ & 300 & 300 & $N$ & 30 & $N$ & 700 & $N$ \\
\hline 422134 & 30 & 20 & 15 & $N$ & 150 & 150 & $N$ & 30 & $N$ & 200 & N \\
\hline 422135 & 30 & 15 & 10 & $N$ & $<100$ & 100 & $N$ & 20 & $N$ & 500 & $N$ \\
\hline 422136 & 30 & 20 & 15 & N & 100 & 100 & $N$ & 30 & $N$ & 500 & $N$ \\
\hline 422137 & 50 & 30 & 30 & $N$ & 300 & 200 & $N$ & 50 & $N$ & 500 & $N$ \\
\hline 422138 & 50 & 30 & 20 & $N$ & 200 & 200 & $N$ & 50 & $N$ & 700 & $N$ \\
\hline 422139 & 50 & 30 & 20 & N & 200 & 200 & $N$ & 50 & $N$ & 700 & $N$ \\
\hline 422140 & 30 & 20 & 20 & $N$ & 300 & 150 & $N$ & 50 & $N$ & 1,000 & $N$ \\
\hline 422141 & 15 & 10 & 10 & $N$ & $\langle 100$ & 100 & $N$ & 15 & $N$ & 150 & $N$ \\
\hline 422142 & 50 & 20 & 20 & $N$ & 300 & 200 & $N$ & 50 & $N$ & 500 & $N$ \\
\hline 422143 & 30 & 30 & 30 & $N$ & 300 & 200 & $N$ & 50 & $N$ & 1,000 & $N$ \\
\hline 422144 & 50 & 20 & 20 & $N$ & 200 & 150 & $N$ & 50 & $N$ & 700 & $N$ \\
\hline
\end{tabular}


TABLE 2.--RESULTS OF SPECTROGRAPHIC ANALYSES OF NURE STREAM-SEDIMENT SAMPLES FROM THE LIVENGOOD AND WESTERN $1 / 3$ OF THE CIRCLE QUADRANGLES, ALASKA--ContinUEd

\begin{tabular}{|c|c|c|c|c|c|c|c|c|c|}
\hline Sample & Latitude & Longitude & $\begin{array}{c}\text { Fe-pet. } \\
5\end{array}$ & $\begin{array}{c}\text { Mg-pet. } \\
5\end{array}$ & $\underset{5}{C a-p c t .}$ & $\begin{array}{c}\text { Ti-pet. } \\
5\end{array}$ & $\begin{array}{c}\text { Mn-pp } \\
5\end{array}$ & $\begin{array}{c}\mathrm{Ag}-\mathrm{pp} \text { 政 } \\
5\end{array}$ & $\begin{array}{c}\text { As-ppa } \\
5\end{array}$ \\
\hline 422145 & 65918 & 1474233 & 1.50 & 2.00 & 5.0 & $\$ 1.000$ & 2,000 & $N$ & $N$ \\
\hline 422146 & $65 \quad 822$ & 1472347 & 10.00 & 2.00 & 5.0 & $>1.000$ & 2,000 & $N$ & N \\
\hline 422147 & 6579 & $14752 \quad 42$ & 10.00 & 2.00 & 5.0 & 31.000 & 1,500 & $N$ & N \\
\hline 422148 & 6559 & 1474023 & 15.00 & 2.00 & 5.0 & 1.000 & 1,500 & $N$ & N \\
\hline 422149 & 65311 & 147433 & 15.00 & 2.00 & 3.0 & 1.000 & 2,000 & $N$ & $N$ \\
\hline 422150 & $65 \quad 154$ & $14749 \quad 19$ & 10.00 & 2.00 & 5.0 & 1.000 & 2,000 & $N$ & N \\
\hline 422152 & 6540 & $147 \quad 48 \quad 24$ & 15.00 & 2.00 & 5.0 & 1.000 & 1,500 & $N$ & $N$ \\
\hline 422153 & 65321 & 1475344 & 7.00 & 1.50 & 3.0 & 1.000 & 1,000 & 1.0 & $N$ \\
\hline 422154 & $65 \quad 159$ & 1475322 & 15.00 & 3.00 & 5.0 & 31.000 & 1,500 & $N$ & $N$ \\
\hline 422155 & $65 \quad 140$ & $148 \quad 0 \quad 5$ & 10.00 & 2.00 & 3.0 & 1.000 & 1,500 & $N$ & $N$ \\
\hline 422156 & $65 \quad 252$ & $148 \quad 0 \quad 30$ & 2.00 & 1.50 & .3 & .200 & 200 & N & $N$ \\
\hline 422157 & $65 \quad 656$ & $148 \quad 0 \quad 47$ & 1.50 & 1.00 & .3 & .200 & 200 & $N$ & $N$ \\
\hline 422158 & $6530 \quad 6$ & 1483458 & 2.00 & 1.50 & .3 & .200 & 200 & N & $N$ \\
\hline 422159 & 652657 & 148370 & 2.00 & 1.00 & .2 & .200 & 200 & $N$ & N \\
\hline 422160 & 652324 & 1483934 & 1.50 & 1.00 & .2 & .150 & 200 & $N$ & $N$ \\
\hline $4221+2$ & 65208 & 149248 & 1.50 & 1.00 & .5 & .200 & 300 & $N$ & N \\
\hline 422163 & 651833 & $149 \quad 557$ & 2.00 & 1.00 & .2 & .200 & 200 & N & N \\
\hline 422164 & 651216 & 1493220 & 1.50 & .50 & .2 & .150 & 200 & $N$ & $N$ \\
\hline 422165 & 65436 & $148 \quad 2545$ & 7.00 & 2.00 & 2.0 & 1.000 & 700 & $<.5$ & $H$ \\
\hline 422166 & $65 \quad 235$ & $148 \quad 939$ & 5.00 & 2.00 & 3.0 & 1.000 & 700 & N & $N$ \\
\hline 422167 & $65 \quad 140$ & $148 \quad 2236$ & 5.00 & 2.00 & 3.0 & 1.000 & 500 & $N$ & $N$ \\
\hline 422168 & 651611 & $148 \quad 50 \quad 47$ & 7.00 & 2.00 & 5.0 & 71.000 & 1,000 & $N$ & $N$ \\
\hline 422169 & 652021 & $14844 \quad 48$ & 5.00 & 1.50 & 2.0 & .700 & 1,000 & $N$ & $N$ \\
\hline 422170 & 65293 & 1484345 & 10.00 & 2.00 & 3.0 & $>1.000$ & 1,000 & $N$ & N \\
\hline 422171 & 653432 & 1482227 & 7.00 & 2.00 & 2.0 & 1.000 & 700 & N & $N$ \\
\hline 422172 & 653432 & $148 \quad 957$ & 5.00 & 1.50 & 3.0 & 1.000 & 700 & $N$ & N \\
\hline 422173 & 653410 & $14810 \quad 4$ & 10.00 & 3.00 & 3.0 & 31.000 & 1,000 & N & $N$ \\
\hline 422174 & 653447 & $148 \quad 230$ & 10.00 & 2.00 & 5.0 & 21.000 & 1,500 & N & $N$ \\
\hline 422175 & 653441 & 1475544 & 7.00 & 2.00 & 5.0 & 71.000 & 1,000 & N & $N$ \\
\hline 422177 & 653418 & $147 \quad 48 \quad 33$ & 7.00 & 2.00 & 5.0 & $>1.000$ & 1,000 & N & $N$ \\
\hline 422178 & 653451 & 1474237 & 7.00 & 1.50 & 3.0 & 1.000 & 700 & N & $N$ \\
\hline 422179 & $6534 \quad 5$ & 1473611 & 7.00 & 2.00 & 5.0 & $>1.000$ & 1,000 & N & N \\
\hline 422180 & 653456 & $14725 \quad 11$ & 10.00 & 2.00 & 5.0 & 1.000 & 1,000 & N & $N$ \\
\hline 422182 & 653430 & $14724 \quad 46$ & 7.00 & 2.00 & 5.0 & 71.000 & 1,500 & $N$ & $N$ \\
\hline 422183 & 653524 & 1472059 & 7.00 & 2.00 & 5.0 & 1.000 & 1,000 & $N$ & $N$ \\
\hline 422184 & 653715 & $147 \quad 1248$ & .70 & 1.00 & 5.0 & .150 & 500 & N & N \\
\hline 422185 & 653733 & $147 \quad 1036$ & 5.00 & 2.00 & 5.0 & 1.000 & 700 & N & $N$ \\
\hline 422187 & 654134 & 14785 & 10.00 & 2.00 & 2.0 & 1.000 & 1,000 & $N$ & $\mathrm{~N}$ \\
\hline 422188 & 653954 & 1473017 & 7.00 & 2.00 & 3.0 & 1.000 & 500 & $N$ & $N$ \\
\hline 422189 & $6537 \quad 8$ & 1473910 & 5.00 & 1.50 & 2.0 & .700 & 500 & $N$ & $N$ \\
\hline 422191 & 653742 & $14756 \quad 48$ & 7.00 & 1.50 & 3.0 & 1.000 & 700 & $N$ & N \\
\hline 422192 & 653750 & 147826 & 7.00 & 2.00 & 3.0 & 1.000 & 700 & $\ddot{N}$ & N \\
\hline 422193 & $6538 \quad 8$ & 1471059 & 7.00 & 2.00 & 5.0 & 1.000 & 1,500 & $N$ & N \\
\hline 422194 & $6528 \quad 10$ & $148 \quad 36 \quad 21$ & 7.00 & 2.00 & 5.0 & 1.000 & 1,000 & N & N \\
\hline 422195 & $6546 \quad 4$ & 149245 & 10.00 & 2.00 & 3.0 & 1.000 & 1,000 & N & N \\
\hline
\end{tabular}


TABLE 2.--PEESULTS OF SPECTROGRAFHIC ANALYSES OF NURE STREAM-SEDIMENT SAMPLES FROM THE LIVENGDOD AND HESTEFN $1 / 3$ OF THE CIRCLE QUADRANGLES, ALASKA--ContinUEd

\begin{tabular}{|c|c|c|c|c|c|c|c|c|c|c|}
\hline Saiple & $\begin{array}{c}\text { B-ppm } \\
s\end{array}$ & $\begin{array}{c}\text { Ba-ppm } \\
s\end{array}$ & $\begin{array}{c}\text { Be-ppm } \\
5\end{array}$ & $\begin{array}{c}B i-p p \text { m } \\
5\end{array}$ & $\begin{array}{c}\text { Co-pp } \\
5\end{array}$ & $\begin{array}{c}\text { Cr-ppm } \\
\mathrm{s}\end{array}$ & $\begin{array}{c}\text { Cu-pp } \\
5\end{array}$ & $\begin{array}{l}\text { La-pp } \\
5\end{array}$ & $\begin{array}{c}\text { Mo-ppm } \\
5\end{array}$ & $\begin{array}{c}N b-p p \text { m } \\
5\end{array}$ \\
\hline 422145 & 100 & 1,500 & 1.0 & $N$ & 20 & 150 & 30 & 50 & $<5$ & 20 \\
\hline 422146 & 150 & 1,000 & 1.0 & $N$ & 20 & 200 & 30 & 70 & $N$ & 20 \\
\hline 422147 & 150 & 1,500 & 1.0 & $N$ & 20 & 200 & 50 & 70 & $N$ & 20 \\
\hline 422148 & 50 & 1,500 & $<1.0$ & $N$ & 20 & 200 & 50 & 50 & N & $N$ \\
\hline 422149 & 100 & 2,000 & 1.5 & $N$ & 30 & 150 & 50 & 50 & $N$ & $N$ \\
\hline 422150 & 100 & 1,500 & 1.0 & $N$ & 20 & 200 & 30 & 50 & $N$ & 20 \\
\hline 422152 & 100 & 2,000 & 1.0 & $N$ & 20 & 200 & 50 & 70 & $N$ & $<20$ \\
\hline 422153 & 100 & 1,500 & 1.0 & $N$ & 15 & 100 & 20 & 50 & 7 & $<20$ \\
\hline 422154 & 100 & 1,500 & $(1.0$ & $N$ & 20 & 200 & 70 & 70 & $N$ & 20 \\
\hline 422155 & 100 & 1,000 & 1.0 & $N$ & 20 & 200 & 50 & 70 & $N$ & $N$ \\
\hline 422156 & 50 & 300 & 01.0 & $N$ & 15 & 100 & 20 & 30 & $N$ & $N$ \\
\hline 422157 & 50 & 500 & 1.0 & $N$ & 15 & 70 & 15 & 30 & $N$ & $N$ \\
\hline 422158 & 50 & 500 & $(1.0$ & $N$ & 15 & 70 & 20 & 30 & N & $N$ \\
\hline 422159 & 50 & 500 & 1.0 & $N$ & 20 & 70 & 30 & 50 & $N$ & $N$ \\
\hline 422160 & 50 & 500 & 1.0 & $N$ & 20 & 50 & 30 & 50 & $N$ & $N$ \\
\hline 422162 & 50 & 500 & 1.0 & $N$ & 15 & 70 & 20 & 20 & $N$ & $N$ \\
\hline 422163 & 50 & 700 & 1.0 & $N$ & 15 & 100 & 15 & 30 & $N$ & $N$ \\
\hline 422164 & 50 & 500 & $\langle 1.0$ & $N$ & 15 & 50 & 20 & 30 & $N$ & $N$ \\
\hline 422165 & 70 & 2,000 & 11.0 & $N$ & 20 & 200 & 50 & 30 & $<5$ & $N$ \\
\hline 422166 & 70 & 1,500 & $\langle 1.0$ & $N$ & 15 & 500 & 50 & 30 & $N$ & $N$ \\
\hline 422167 & 70 & 2,000 & $<1.0$ & $N$ & 15 & 300 & 70 & 50 & $<5$ & $N$ \\
\hline 422168 & 70 & 2,000 & $<1.0$ & $N$ & 15 & 500 & 50 & 50 & $N$ & $<20$ \\
\hline 422169 & 50 & 1,500 & $\langle 1.0$ & $N$ & 10 & 100 & 30 & $N$ & $N$ & $N$ \\
\hline 422170 & 100 & 2,000 & 1.0 & $N$ & 20 & 500 & 70 & 30 & $N$ & 20 \\
\hline 422171 & 70 & 2,000 & 1.0 & $N$ & 20 & 300 & 70 & 30 & $N$ & $N$ \\
\hline 422172 & 50 & 2,000 & 1.0 & $N$ & 10 & 70 & 50 & $\mathrm{~N}$ & $<5$ & $N$ \\
\hline 422173 & 100 & 3,000 & 1.0 & $N$ & 20 & 200 & 70 & 50 & $N$ & $<20$ \\
\hline 422174 & 100 & 3,000 & 1.0 & $N$ & 20 & 300 & 70 & 50 & $N$ & $<20$ \\
\hline 422175 & 70 & 2,000 & $\langle 1.0$ & $N$ & 15 & 300 & 50 & 50 & $N$ & $<20$ \\
\hline 422177 & 70 & 3,000 & 1.0 & $N$ & 20 & 300 & 70 & 50 & $N$ & 20 \\
\hline 422178 & 70 & 2,000 & 1.0 & $N$ & 20 & 300 & 50 & 30 & $N$ & $N$ \\
\hline 422179 & 100 & 3,000 & $\langle 1.0$ & $N$ & 20 & 500 & 50 & 50 & $N$ & $<20$ \\
\hline 422180 & 70 & 3,000 & $\langle 1.0$ & $N$ & 20 & 500 & 100 & 50 & 5 & $<20$ \\
\hline 422182 & 100 & 3,000 & $\langle 1.0$ & $N$ & 20 & 300 & 50 & 50 & $N$ & $\measuredangle 20$ \\
\hline 422183 & 100 & 3,000 & $\langle 1.0$ & $N$ & 20 & 300 & 50 & 50 & $N$ & $N$ \\
\hline 422184 & 10 & 500 & 41.0 & $N$ & 5 & 20 & 20 & $N$ & $N$ & $N$ \\
\hline 422185 & 100 & 2,000 & 1.0 & $N$ & 20 & 300 & 50 & 50 & $<5$ & $<20$ \\
\hline 422187 & 150 & 2,000 & 1.0 & $N$ & 20 & 300 & 30 & 70 & $<5$ & $\langle 20$ \\
\hline 422188 & 70 & 1,000 & $\langle 1.0$ & $N$ & 20 & 200 & 20 & 50 & $N$ & $<20$ \\
\hline 422189 & 70 & 1,500 & $\langle 1.0$ & $N$ & 15 & 150 & 15 & 50 & N & 20 \\
\hline 422191 & 70 & 1,500 & 1.0 & $N$ & 10 & 200 & 20 & 50 & $N$ & $<20$ \\
\hline 422192 & 70 & 1,500 & $\$ 1.0$ & $N$ & 15 & 200 & 30 & 50 & $\langle 5$ & 20 \\
\hline 422193 & 70 & 1,500 & $\langle 1.0$ & $N$ & 15 & 200 & 15 & 50 & $N$ & $<20$ \\
\hline 422194 & 70 & 1,500 & 1.0 & $N$ & 15 & 200 & 50 & 30 & $N$ & $N$ \\
\hline 422195 & 70 & 1,500 & $\langle 1.0$ & $N$ & 20 & 200 & 70 & 30 & $N$ & $<20$ \\
\hline
\end{tabular}


TABLE 2. - RESULTS OF SPECTROGRAPHIC ANALYSES OF NUAE STREAM-SEDIMENT SAMPLES FROM THE LIVENGOOD

AND WESTERN $1 / 3$ OF THE CIRCLE QUADRANGLES, ALASKA- ContinUEd

\begin{tabular}{|c|c|c|c|c|c|c|c|c|c|c|c|}
\hline Sa桶le & $\begin{array}{c}\mathrm{Hi}-\mathrm{pp} \text { i } \\
5\end{array}$ & $\begin{array}{c}\mathrm{Pb}-\mathrm{pp} \text { 估 } \\
5\end{array}$ & $\begin{array}{c}S c-p p \text { 促 } \\
5\end{array}$ & $\begin{array}{c}S n-p p \text { m } \\
5\end{array}$ & $\begin{array}{c}\mathrm{Sr}-\mathrm{pp} \text { \# } \\
5\end{array}$ & $\begin{array}{c}V-p p d \\
5\end{array}$ & $\begin{array}{c}H-p p i n \\
5\end{array}$ & $\begin{array}{c}Y-p p \text { a } \\
5\end{array}$ & $\begin{array}{c}2 n-p p \text { a } \\
5\end{array}$ & $\begin{array}{c}2 r-p p m \\
5\end{array}$ & $\begin{array}{c}\text { Th-pp } \\
5\end{array}$ \\
\hline 422145 & 50 & 30 & 20 & $H$ & 200 & 200 & $N$ & 50 & $N$ & 500 & $N$ \\
\hline 422146 & 50 & 20 & 30 & H & 300 & 150 & $N$ & 50 & N & 700 & $N$ \\
\hline 422147 & 50 & 20 & 30 & $H$ & 300 & 200 & $H$ & 50 & $N$ & 1,000 & $H$ \\
\hline 422148 & 50 & 50 & 20 & N & 200 & 200 & $N$ & 50 & $N$ & 500 & $N$ \\
\hline 422149 & 70 & 50 & 20 & N & 200 & 200 & $\mathrm{~N}$ & 50 & $N$ & 300 & $\mathrm{~N}$ \\
\hline 422150 & 50 & 30 & 20 & H & 300 & 200 & $N$ & 50 & $N$ & 700 & $N$ \\
\hline 422152 & 70 & 30 & 20 & $N$ & 200 & 300 & $N$ & 50 & $N$ & 500 & $N$ \\
\hline 422153 & 30 & 20 & 15 & W & 200 & 150 & $N$ & 50 & $N$ & 1,000 & $H$ \\
\hline 422154 & 70 & 30 & 20 & $H$ & 200 & 300 & $H$ & 50 & $H$ & 500 & $N$ \\
\hline 422155 & 70 & 50 & 20 & $N$ & 200 & 300 & $H$ & 50 & $N$ & 300 & $N$ \\
\hline 422156 & 50 & 15 & 15 & N & 100 & 70 & $N$ & 20 & N & 150 & H \\
\hline 422157 & 30 & 10 & 7 & $N$ & 100 & 70 & $N$ & 15 & $H$ & 150 & H \\
\hline 422158 & 50 & 15 & 10 & $H$ & 150 & 70 & $n$ & 20 & $H$ & 100 & $N$ \\
\hline 422159 & 50 & 20 & 10 & $N$ & 100 & 100 & $N$ & 20 & $N$ & 150 & $N$ \\
\hline 422160 & 50 & 15 & 10 & $N$ & 100 & 70 & $n$ & 20 & $N$ & 100 & $H$ \\
\hline 422162 & 70 & 10 & 7 & $N$ & 150 & 70 & $N$ & 15 & $N$ & 100 & $n$ \\
\hline 422163 & 50 & 15 & 10 & N & 100 & 100 & $N$ & 20 & $N$ & 100 & $n$ \\
\hline 422164 & 50 & 15 & 7 & $H$ & $\longleftrightarrow 100$ & 70 & $N$ & 20 & $N$ & 100 & $H$ \\
\hline 422165 & 50 & 50 & 20 & N & 200 & 200 & $H$ & 20 & $<200$ & 200 & 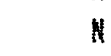 \\
\hline 422166 & 50 & 20 & 20 & H & 200 & 150 & $N$ & 20 & 200 & 500 & $\mathrm{~N}$ \\
\hline 422167 & 50 & 20 & 20 & $N$ & 200 & 200 & $N$ & 30 & $N$ & 300 & $H$ \\
\hline 422168 & 50 & 20 & 20 & $n$ & 200 & 200 & $N$ & 50 & H & 500 & N \\
\hline 422169 & 50 & 15 & 10 & H & 100 & 150 & $N$ & 20 & N & 150 & $H$ \\
\hline 422170 & 50 & 30 & 20 & $N$ & 200 & 300 & $N$ & 50 & H & 500 & $H$ \\
\hline 422171 & 50 & 20 & 20 & $N$ & 200 & 200 & $N$ & 30 & $H$ & 300 & $N$ \\
\hline 422172 & 50 & 15 & 15 & $N$ & 150 & 150 & $N$ & 20 & $N$ & 150 & $N$ \\
\hline 422173 & 50 & 30 & 30 & $\mathrm{H}$ & 300 & 200 & $N$ & 50 & $<200$ & 300 & $H$ \\
\hline 422174 & 50 & 50 & 30 & $\mathrm{H}$ & 300 & 300 & $H$ & 50 & $N$ & 300 & $N$ \\
\hline 422175 & 30 & 20 & 20 & N & 300 & 200 & $H$ & 50 & $N$ & 700 & $H$ \\
\hline 422177 & 50 & 30 & 30 & $H$ & 300 & 200 & $H$ & 50 & N & 300 & $N$ \\
\hline 422178 & 50 & 20 & 20 & $H$ & 200 & 150 & $N$ & 30 & $N$ & 500 & $N$ \\
\hline 422179 & 30 & 20 & 30 & $N$ & 300 & 300 & $N$ & 50 & $\mathrm{H}$ & 700 & $H$ \\
\hline 422180 & 50 & 30 & 30 & $H$ & 300 & 300 & $H$ & 50 & H & 300 & $N$ \\
\hline 422182 & 50 & 20 & 20 & $N$ & 300 & 300 & $\mathrm{~N}$ & 50 & $H$ & 500 & $H$ \\
\hline 422183 & 50 & 30 & 20 & $N$ & 300 & 300 & $n$ & 50 & $n$ & 300 & $N$ \\
\hline 422184 & 30 & $<10$ & 7 & N & $N$ & 70 & $H$ & 10 & $N$ & 50 & $N$ \\
\hline 422185 & 50 & 20 & 20 & N & 300 & 200 & $n$ & 50 & $N$ & 700 & $N$ \\
\hline 422187 & 50 & 30 & 30 & $N$ & 200 & 150 & $N$ & 50 & $N$ & 500 & $N$ \\
\hline 422188 & 30 & 20 & 20 & H & 300 & 150 & $N$ & 30 & $N$ & 200 & $\mathrm{H}$ \\
\hline 422189 & 30 & 20 & 20 & N & 300 & 150 & $n$ & 30 & 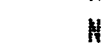 & 300 & $N$ \\
\hline 422191 & 30 & 20 & 30 & $n$ & 300 & 200 & $H$ & 30 & $\#$ & 300 & $N$ \\
\hline 422192 & 30 & 20 & 20 & $\ddot{N}$ & 300 & 150 & $H$ & 30 & $N$ & 300 & $H$ \\
\hline 422193 & 30 & 15 & 20 & $N$ & 300 & 150 & $N$ & 50 & $N$ & 300 & $N$ \\
\hline 422194 & 30 & 20 & 20 & $N$ & 300 & 100 & $N$ & 20 & H & 300 & $\mathrm{~N}$ \\
\hline 422195 & 50 & 20 & 20 & $\mathrm{H}$ & 200 & 200 & $N$ & 50 & $\|$ & 300 & $H$ \\
\hline
\end{tabular}


TABLE 2.--RESULTS OF SPECTROGRAPHIC ANALYSES OF NURE STREAM-SEDIMENT SAMPLES FROM THE LIVENGDOD AND WESTERN $1 / 3$ OF THE CIRCLE QUADRANGLES, ALASKA--ContinUEd

\begin{tabular}{|c|c|c|c|c|c|c|c|c|c|}
\hline Sample & Latitude & Longitude & $\begin{array}{c}\text { Fe-pct. } \\
s\end{array}$ & $\begin{array}{c}M g-p c t . \\
s\end{array}$ & $\begin{array}{c}\text { Ca-pct. } \\
s\end{array}$ & $\begin{array}{c}\text { Ti-pct. } \\
s\end{array}$ & $\begin{array}{c}\text { Mn-ppm } \\
5\end{array}$ & $\begin{array}{c}\text { Ag-pp省 } \\
5\end{array}$ & $\begin{array}{c}A 5-p p m \\
5\end{array}$ \\
\hline 422196 & 655143 & 1493520 & 5.00 & 2.00 & 5.0 & 1.000 & 1,000 & N & $N$ \\
\hline 422197 & $6548 \quad 8$ & 1492553 & 5.00 & 1.50 & 3.0 & $>1.000$ & 1,000 & $H$ & $N$ \\
\hline 422198 & 654536 & 1492120 & 5.00 & 2.00 & 3.0 & 1.000 & 1,000 & N & N \\
\hline 422199 & 654456 & 14920 & .50 & .50 & 3.0 & .150 & 150 & $N$ & $N$ \\
\hline 422200 & 654422 & 1491726 & 5.00 & 1.50 & 1.0 & .700 & 300 & $N$ & $N$ \\
\hline 422201 & 654345 & 1491245 & 7.00 & 2.00 & 3.0 & 1.000 & 700 & $N$ & $N$ \\
\hline 422202 & 653821 & 149311 & 7.00 & 2.00 & 3.0 & .700 & 700 & N & N \\
\hline 422203 & $6534 \quad 4$ & 1485557 & 5.00 & 1.50 & 3.0 & .700 & 700 & $N$ & $n$ \\
\hline 422204 & 65341 & $14856 \quad 29$ & 7.00 & 2.00 & 3.0 & 1.000 & 1,000 & N & $\mathrm{N}$ \\
\hline 422205 & 653051 & 1484950 & 3.00 & 1.50 & 1.5 & .700 & 300 & $N$ & $N$ \\
\hline 422206 & 65319 & $148 \quad 4731$ & 7.00 & 2.00 & 3.0 & 1.000 & 700 & N & $N$ \\
\hline 422207 & 65539 & 1492656 & 7.00 & 2.00 & 3.0 & 1.000 & 700 & $N$ & $N$ \\
\hline 422200 & 652232 & $14839 \quad 15$ & 7.00 & 2.00 & 3.0 & 1.000 & 1,000 & N & N \\
\hline 422209 & 651946 & 1483956 & 10.00 & 3.00 & 3.0 & 1.000 & 1,500 & $H$ & N \\
\hline 422210 & 651726 & 1484115 & 10.00 & 2.00 & 5.0 & 1.000 & 1,000 & $N$ & $N$ \\
\hline 422211 & 65148 & $148 \quad 42 \quad 15$ & 15.00 & 2.00 & 3.0 & 1.000 & 2,000 & $N$ & N \\
\hline 422212 & 651157 & 1484329 & 10.00 & 2.00 & 3.0 & 1.000 & 1,000 & N & $N$ \\
\hline 422213 & 651228 & 1483559 & 15.00 & 2.00 & 5.0 & 1.000 & 2,000 & N & $N$ \\
\hline 422214 & 651353 & $148 \quad 2841$ & 10.00 & 2.00 & 5.0 & 1.000 & 5,000 & N & $N$ \\
\hline 422215 & $6516 \quad 14$ & 1482912 & 1.50 & 1.00 & 3.0 & .300 & 500 & $N$ & $N$ \\
\hline 422216 & 651552 & 1482314 & 15.00 & 3.00 & 5.0 & 1.000 & 1,500 & N & $N$ \\
\hline 422217 & 65151 & 1482059 & 7.60 & 1.50 & 5.0 & .700 & 1,000 & N & N \\
\hline 422218 & 651336 & $14826 \quad b$ & 7.00 & 2.00 & 7.0 & 1.000 & 1,500 & $N$ & N \\
\hline 422219 & 651125 & $14826 \quad 12$ & 7.00 & 1.50 & 3.0 & 1.000 & 700 & $N$ & H \\
\hline 422221 & 651050 & 148313 & 7.00 & 2.00 & 3.0 & 1.000 & 700 & N & $N$ \\
\hline 422222 & $65 \quad 8 \quad 12$ & 1482854 & 7.00 & 1.50 & 3.0 & 1.000 & 700 & $N$ & $N$ \\
\hline 422223 & 65838 & 1483518 & 10.00 & 2.00 & 5.0 & .700 & 1,500 & N & $N$ \\
\hline 422224 & 65712 & $14844 \quad 49$ & 10.00 & 2.00 & 5.0 & 1.000 & 1,500 & N & N \\
\hline 422225 & $65 \quad 633$ & 1483750 & 10.00 & 2.00 & 5.0 & 1.000 & 1,500 & N & $N$ \\
\hline 422226 & 6573 & 1483156 & 7.00 & 2.00 & 5.0 & 1.000 & 1,000 & $N$ & $N$ \\
\hline 422227 & $65 \quad 442$ & $148 \quad 30 \quad 47$ & 7.00 & 1.50 & 3.0 & 1.000 & 1,000 & N & $N$ \\
\hline 422228 & 65520 & $14826 \quad 45$ & 7.00 & 3.00 & 7.0 & .700 & 1,500 & N & N \\
\hline 422230 & 6575 & 1481816 & 7.00 & 2.00 & 2.0 & 1.000 & 1,500 & N & N \\
\hline 422231 & 65315 & $148 \quad 615$ & 7.00 & 1.50 & 2.0 & 1.000 & 1,000 & $N$ & $N$ \\
\hline 422232 & 65156 & $148 \quad 620$ & 5.00 & 1.50 & 3.0 & 1.000 & 1,000 & N & N \\
\hline 422233 & $65 \quad 28$ & 1481143 & 5.00 & 1.50 & 2.0 & 1.000 & 1,000 & $N$ & N \\
\hline 422234 & $65 \quad 132$ & 1481919 & 7.00 & 2.00 & 5.0 & 1.000 & 1,000 & N & $N$ \\
\hline 422235 & 65128 & $14824 \quad 1$ & 7.00 & 3.00 & 7.0 & 1.000 & 1,500 & $N$ & $N$ \\
\hline 422236 & 65117 & $148 \quad 29 \quad 12$ & 7.00 & 2.00 & 5.0 & .700 & 1,500 & $N$ & $N$ \\
\hline 422237 & $65 \quad 159$ & 1483844 & 7.00 & 2.00 & 3.0 & 1.000 & 1,500 & $N$ & $N$ \\
\hline 422238 & $65 \quad 249$ & $148 \quad 38 \quad 20$ & 5.00 & 1.50 & 5.0 & 1.000 & 1,000 & N & N \\
\hline 422239 & 65041 & 1484210 & 15.00 & 2.00 & .2 & 1.000 & 1,000 & $N$ & $N$ \\
\hline $422240^{\circ}$ & 65158 & 1484853 & 10.00 & 3.00 & 7.0 & 1.000 & 1,000 & N & N \\
\hline 422241 & 65150 & 148539 & 7.00 & 1.50 & 2.0 & 1.000 & 500 & N & $N$ \\
\hline 422242 & $65 \quad 347$ & 1485528 & 7.00 & 2.00 & 7.0 & 1.000 & 700 & N & N \\
\hline
\end{tabular}


TABLE 2.--RESULTS OF SPECTROGRAPHIC ANALYSES OF NURE STREAM-SEDIMENT SAMPLES FROM THE LIVENGOOD AND WESTERN $1 / 3$ DF THE CIRCLE gUADRANGLES, ALASKA--Continued

\begin{tabular}{|c|c|c|c|c|c|c|c|c|c|c|}
\hline Sample & $\begin{array}{c}\text { B-ppo } \\
5\end{array}$ & $\begin{array}{c}\mathrm{Ba}-\mathrm{pp} \\
5\end{array}$ & $\begin{array}{c}\text { Be-ppi } \\
5\end{array}$ & $\begin{array}{c}B i-p p \text { i } \\
5\end{array}$ & $\begin{array}{c}\text { Co-ppi } \\
5\end{array}$ & $\begin{array}{c}\text { Cr-ppi } \\
5\end{array}$ & $\begin{array}{c}\text { Cu-ppin } \\
s\end{array}$ & $\begin{array}{c}\text { La-pp } \\
5\end{array}$ & $\begin{array}{c}\text { Mo-pp } \\
5\end{array}$ & $\begin{array}{c}\mathrm{Nb}-\mathrm{pp} \text { m } \\
5\end{array}$ \\
\hline 422196 & 50 & 1,000 & 1.0 & $N$ & 10 & 150 & 15 & 30 & $N$ & N \\
\hline 422197 & 70 & 700 & $<1.0$ & $N$ & 10 & 200 & 50 & 30 & $<5$ & $<20$ \\
\hline 422198 & 70 & 1,000 & 1.0 & $N$ & 15 & 200 & 20 & 30 & N & $N$ \\
\hline 422199 & $<10$ & 300 & 1.0 & $N$ & $N$ & 10 & 20 & $N$ & $N$ & $N$ \\
\hline 422200 & 50 & 700 & $\langle 1.0$ & $N$ & 15 & 200 & 30 & 30 & $N$ & $N$ \\
\hline 422201 & 70 & 1,500 & 1.0 & $N$ & 10 & 150 & 30 & 50 & $N$ & $<20$ \\
\hline 422202 & 70 & 1,500 & 1.0 & $N$ & 20 & 200 & 50 & 50 & $N$ & $\langle 20$ \\
\hline 422203 & 50 & 1,500 & $\langle 1.0$ & $N$ & 15 & 200 & 70 & 30 & $N$ & $N$ \\
\hline 422204 & 70 & 1,500 & $\langle 1.0$ & $N$ & 20 & 200 & 50 & 50 & $N$ & N \\
\hline 422205 & 50 & 700 & $\langle 1.0$ & $N$ & 10 & 70 & 15 & 30 & $N$ & $H$ \\
\hline 422206 & 70 & 2,000 & 1.0 & $N$ & 15 & 200 & 50 & 50 & $N$ & $N$ \\
\hline 422207 & 70 & 2,000 & 1.0 & $N$ & 20 & 200 & 50 & 50 & $N$ & $<20$ \\
\hline 422200 & 70 & 2,000 & 1.0 & $N$ & 20 & 150 & 30 & 50 & $<5$ & $N$ \\
\hline 422209 & 70 & 1,500 & $\langle 1.0$ & $N$ & 15 & 300 & 50 & 50 & $N$ & $<20$ \\
\hline 422210 & 70 & 2,000 & 1.0 & $N$ & 15 & 200 & 50 & 70 & $N$ & N \\
\hline 422211 & 70 & 2,000 & $\langle 1.0$ & $N$ & 15 & 300 & 100 & 30 & $<5$ & $N$ \\
\hline 422212 & 70 & 2,000 & $<1.0$ & $N$ & 20 & 200 & 50 & 70 & $N$ & $<20$ \\
\hline 422213 & 70 & 1,500 & $N$ & $N$ & 10 & 300 & 30 & 50 & $N$ & N \\
\hline 422214 & 100 & 2,000 & 1.5 & $N$ & 15 & 300 & 50 & 70 & $N$ & $<20$ \\
\hline 422215 & 20 & 700 & 1.0 & $N$ & 7 & 20 & 20 & $N$ & $N$ & $N$ \\
\hline 422216 & 70 & 2,000 & $N$ & $H$ & 15 & 200 & 70 & 20 & $N$ & $<20$ \\
\hline 422217 & 100 & 2,000 & $\langle 1.0$ & $N$ & 15 & 200 & 20 & 50 & $N$ & $<20$ \\
\hline 422218 & 70 & 2,000 & $(1.0$ & $N$ & 15 & 200 & 30 & 50 & $N$ & $<20$ \\
\hline 422219 & 70 & 1,500 & $\langle 1.0$ & $N$ & 10 & 300 & 20 & 50 & $<5$ & $<20$ \\
\hline 422221 & 100 & 2,000 & 41.0 & $N$ & 15 & 200 & 50 & 50 & $N$ & $N$ \\
\hline 422222 & 70 & 2,000 & $\langle 1.0$ & $N$ & 10 & 150 & 30 & 50 & $N$ & $<20$ \\
\hline 422223 & 100 & 2,000 & $\langle 1.0$ & $N$ & 20 & 200 & 100 & 30 & $<5$ & $N$ \\
\hline 422224 & 70 & 2,000 & $<1.0$ & $N$ & 15 & 200 & 30 & 50 & N & $<20$ \\
\hline 422225 & 70 & 2,000 & $\langle 1.0$ & $N$ & 20 & 200 & 50 & 30 & $<5$ & 120 \\
\hline 422226 & 70 & 2,000 & $\langle 1.0$ & $N$ & 15 & 300 & 20 & 50 & $\langle 5$ & $<20$ \\
\hline 422227 & 100 & 2,000 & $(1.0$ & $N$ & 15 & 150 & 20 & 50 & $N$ & $<20$ \\
\hline 422228 & 100 & 2,000 & $\langle 1.0$ & $N$ & 20 & 500 & 50 & 50 & $N$ & $<20$ \\
\hline 422230 & 100 & 2,000 & 1.0 & $N$ & 15 & 200 & 20 & 50 & $N$ & (20) \\
\hline 422231 & 70 & 1,500 & $\langle 1.0$ & $N$ & 20 & 200 & 15 & 20 & $N$ & $<20$ \\
\hline 422232 & 70 & 2,000 & $\$ 1.0$ & H & 15 & 150 & 20 & 30 & $N$ & $<20$ \\
\hline 422233 & 70 & 1,000 & $\langle 1.0$ & $N$ & 15 & 200 & 15 & 20 & $N$ & 20 \\
\hline 422234 & 70 & 1,500 & $\langle 1.0$ & $N$ & 15 & 200 & 30 & 30 & N & $N$ \\
\hline 422235 & 70 & 2,000 & 1.0 & $N$ & 20 & 300 & 50 & 70 & $N$ & $<20$ \\
\hline 422236 & 50 & 2,000 & $\langle 1.0$ & $N$ & 20 & 200 & 30 & 50 & N & $<20$ \\
\hline 422237 & 70 & 2,000 & 1.0 & $N$ & 20 & 200 & 50 & 50 & $N$ & $<20$ \\
\hline 422238 & 70 & 2,000 & $\langle 1.0$ & $N$ & 15 & 200 & 30 & 50 & $N$ & $<20$ \\
\hline 422239 & 50 & 2,000 & $\langle 1.0$ & $N$ & 20 & 300 & 50 & 20 & $<5$ & $<20$ \\
\hline 422240 & 70 & 2,000 & $\langle 1.0$ & $N$ & 20 & 300 & 30 & 70 & 45 & $<20$ \\
\hline 422241 & 70 & 1,500 & $<1.0$ & $N$ & 15 & 300 & 30 & 50 & $N$ & $<20$ \\
\hline 422242 & 70 & 2,000 & $\langle 1.0$ & $N$ & 15 & 200 & 30 & 50 & $N$ & $N$ \\
\hline
\end{tabular}


TABLE 2.--RESULTS OF SPECTROGRAPHIC ANALYSES OF NURE STREAM-SEDIMENT SAMPLES FROH THE LIVENGOOD

AND WESTERN $1 / 3$ OF THE CIRCLE QUADRANGLES, ALASKA--ContinUEd

\begin{tabular}{|c|c|c|c|c|c|c|c|c|c|c|c|}
\hline Sample & $\begin{array}{c}\mathrm{Ni}-\mathrm{pp} \\
\mathrm{s}\end{array}$ & $\begin{array}{c}\text { Pb-ppi } \\
5\end{array}$ & $\begin{array}{c}\text { St-ppm } \\
5\end{array}$ & $\begin{array}{c}5 n-p p \text { m } \\
3\end{array}$ & $\begin{array}{c}\mathrm{Gr}-\mathrm{pp} \\
5\end{array}$ & $\begin{array}{c}V-p p h \\
5\end{array}$ & $\begin{array}{c}\text { H-ppi } \\
5\end{array}$ & $\begin{array}{c}Y-p p \text { i } \\
5\end{array}$ & $\begin{array}{c}2 n-p p m \\
5\end{array}$ & $\begin{array}{c}\text { Ir-ppa } \\
s\end{array}$ & $\begin{array}{c}\text { Th-pp } \\
5\end{array}$ \\
\hline 422196 & 20 & 15 & 20 & $N$ & 300 & 100 & $N$ & 20 & $N$ & 300 & $N$ \\
\hline 422197 & 30 & 15 & 20 & $N$ & 200 & 150 & $N$ & 30 & $N$ & 500 & $N$ \\
\hline 422198 & 30 & 20 & 20 & $N$ & 200 & 150 & $N$ & 30 & $N$ & 500 & $N$ \\
\hline 422199 & 15 & 10 & $\langle 5$ & $N$ & $N$ & 70 & $N$ & 15 & $N$ & 70 & $N$ \\
\hline 422200 & 30 & 20 & 10 & $N$ & 150 & 100 & $N$ & 20 & N & 150 & $N$ \\
\hline 422201 & 30 & 20 & 20 & $N$ & 200 & 200 & $N$ & 30 & $N$ & 300 & $N$ \\
\hline 422202 & 30 & 20 & 20 & $N$ & 300 & 200 & $N$ & 30 & $\langle 200$ & 200 & $N$ \\
\hline 422203 & 30 & 20 & 20 & $N$ & 300 & 150 & $\mathbf{N}$ & 30 & $N$ & 200 & $N$ \\
\hline 422204 & 50 & 20 & 20 & $N$ & 300 & 200 & $N$ & 30 & $N$ & 300 & $N$ \\
\hline 422205 & 30 & 10 & 10 & $N$ & 150 & 150 & $N$ & 20 & $N$ & 150 & $N$ \\
\hline 422206 & 70 & 20 & 20 & $N$ & 300 & 200 & N & 50 & $N$ & 200 & $N$ \\
\hline 4222007 & 50 & 20 & 20 & $N$ & 300 & 200 & $N$ & 50 & $N$ & 300 & $N$ \\
\hline 422208 & 50 & 20 & 20 & $N$ & 300 & 200 & $N$ & 50 & $N$ & 300 & $N$ \\
\hline 422209 & 30 & 20 & 20 & $N$ & 300 & 300 & $N$ & 30 & $N$ & 300 & $N$ \\
\hline 422210 & 50 & 20 & 20 & $N$ & 300 & 150 & $N$ & 50 & $N$ & 200 & $N$ \\
\hline 422211 & 50 & 20 & 20 & $N$ & 200 & 300 & $N$ & 30 & $N$ & 200 & $N$ \\
\hline 422212 & 50 & 20 & 30 & $N$ & 300 & 300 & $N$ & 50 & $N$ & 300 & $N$ \\
\hline 422213 & 20 & 30 & 15 & $N$ & 500 & 500 & $N$ & 20 & $N$ & 1,000 & $N$ \\
\hline 422214 & 50 & 30 & 20 & $N$ & 700 & 200 & 100 & 50 & $N$ & 700 & $N$ \\
\hline 422215 & 30 & $<10$ & 10 & $N$ & $\langle 100$ & 100 & $N$ & 20 & N & 70 & $N$ \\
\hline 422216 & 50 & 20 & 20 & $N$ & 300 & 500 & $N$ & 20 & $N$ & 500 & $N$ \\
\hline 422217 & 30 & 30 & 20 & $N$ & 500 & 150 & $N$ & 30 & N & 300 & $N$ \\
\hline 422218 & 50 & 20 & 20 & $N$ & 500 & 300 & $N$ & 30 & $N$ & 300 & $N$ \\
\hline 422219 & 30 & 15 & 20 & $N$ & 300 & 200 & $N$ & 50 & $N$ & 700 & $N$ \\
\hline 422221 & 70 & 15 & 20 & $N$ & 300 & 200 & $N$ & 50 & $N$ & 300 & $N$ \\
\hline 422222 & 20 & 20 & 20 & $N$ & 300 & 150 & $N$ & 50 & N & 300 & $N$ \\
\hline 422223 & 50 & 15 & 20 & $N$ & 300 & 300 & $N$ & 50 & N & 300 & $N$ \\
\hline 422224 & 30 & 15 & 20 & $N$ & 500 & 200 & $N$ & 50 & N & 1,000 & $N$ \\
\hline 422225 & 30 & 20 & 20 & $N$ & 500 & 200 & $N$ & 50 & N & 500 & N \\
\hline 422226 & 30 & 15 & 20 & $N$ & 500 & 200 & $N$ & 50 & $N$ & 500 & $N$ \\
\hline 422227 & 20 & 15 & 20 & 20 & 500 & 150 & $N$ & 50 & $N$ & 300 & N \\
\hline 422228 & 50 & 20 & 20 & $N$ & 500 & 150 & $N$ & 50 & $N$ & 300 & $N$ \\
\hline 422230 & 30 & 20 & 20 & $N$ & 200 & 200 & $N$ & 50 & $N$ & 300 & $N$ \\
\hline 422231 & 30 & 20 & 20 & $N$ & 200 & 150 & $N$ & 30 & $N$ & 500 & $N$ \\
\hline 422232 & 30 & 20 & 20 & $N$ & 300 & 150 & $N$ & 30 & $N$ & 300 & $N$ \\
\hline 422233 & 30 & 20 & 10 & $N$ & 200 & 150 & $N$ & 30 & $N$ & 300 & $N$ \\
\hline 422234 & 50 & 20 & 20 & $H$ & 300 & 150 & $N$ & 30 & $N$ & 200 & $N$ \\
\hline 422235 & 50 & 20 & 30 & $N$ & 500 & 200 & $N$ & 30 & $N$ & 500 & $H$ \\
\hline $42223 b$ & 50 & 20 & 20 & $N$ & 300 & 200 & $N$ & 50 & $N$ & 300 & $N$ \\
\hline 422237 & 50 & 50 & 20 & $N$ & 200 & 150 & $N$ & 50 & $N$ & 300 & $N$ \\
\hline 422238 & 50 & 20 & 20 & $N$ & 300 & 200 & $N$ & 50 & $N$ & 500 & $N$ \\
\hline 422239 & 30 & 50 & 15 & $N$ & $\$ 100$ & 150 & $N$ & 20 & $N$ & 500 & N \\
\hline 422240 & 50 & 20 & 20 & $N$ & 500 & 200 & $N$ & 50 & $N$ & 500 & $N$ \\
\hline 422241 & 30 & 30 & 15 & $N$ & 200 & 100 & $N$ & 30 & $N$ & 300 & $N$ \\
\hline 422242 & 50 & 20 & 20 & $N$ & 500 & 150 & $N$ & 50 & $N$ & 500 & $N$ \\
\hline
\end{tabular}


TABLE 2.--RESULTS OF SPECTROGRAPHIC ANALYSES OF NURE STREAM-SEDIMENT SAMPLES FROM THE LIVENGOOD AND WESTERN $1 / 3$ OF THE CIRCLE QUADRANGLES, ALASKA--Continued

\begin{tabular}{|c|c|c|c|c|c|c|c|c|c|}
\hline Sample & Latitude & Longitude & $\begin{array}{c}\text { Fe-pct. } \\
5\end{array}$ & $\begin{array}{c}\text { Mg-pct. } \\
5\end{array}$ & $\begin{array}{c}\text { Ca-pct. } \\
5\end{array}$ & $\begin{array}{c}\text { Ti-pct. } \\
5\end{array}$ & $\begin{array}{c}\text { Mn-ppi } \\
s\end{array}$ & $\begin{array}{c}A g-p p \\
5\end{array}$ & $\begin{array}{c}\text { As-ppi } \\
s\end{array}$ \\
\hline 422243 & 65320 & 1484918 & 10.00 & 1.50 & 2.0 & .700 & 5,000 & N & $N$ \\
\hline 422244 & 65442 & $148 \quad 4431$ & 7.00 & 1.50 & 2.0 & 1.000 & 2,000 & $N$ & N \\
\hline 422245 & $65 \quad 619$ & 1484751 & 15.00 & 3.00 & 5.0 & 31.000 & 1,500 & N & $N$ \\
\hline 422247 & 65527 & 1485325 & 7.00 & 2.00 & 3.0 & 1.000 & 700 & $N$ & N \\
\hline 422248 & 65938 & 1485333 & 7.00 & 3.00 & 5.0 & 1.000 & 1,500 & $N$ & $N$ \\
\hline 422249 & 651028 & 1485338 & 5.00 & 1.50 & 2.0 & 1.000 & 500 & $N$ & N \\
\hline 422250 & $65 \quad 928$ & $148 \quad 48 \quad 11$ & 7.00 & 1.50 & 5.0 & 1.000 & 1,000 & $N$ & $N$ \\
\hline 422251 & 651211 & 1485053 & 7.00 & 2.00 & 2.0 & .700 & 1,000 & $N$ & $N$ \\
\hline 422252 & 65130 & 1485129 & 7.00 & 2.00 & 5.0 & 1.000 & 1,500 & N & N \\
\hline 422253 & 651815 & 1484541 & 7.00 & 2.00 & 5.0 & 1.000 & 1,000 & $N$ & $N$ \\
\hline 422254 & 652124 & $148 \quad 4454$ & 5.00 & 2.00 & 5.0 & 1.000 & 1,000 & N & N \\
\hline 422255 & 652156 & $14850 \quad 8$ & 10.00 & 2.00 & 5.0 & 1.000 & 1,500 & $N$ & $N$ \\
\hline 422256 & 652326 & $14848 \quad 42$ & 7.00 & 2.00 & 5.0 & 1.000 & 1,000 & N & $N$ \\
\hline 422257 & $6526 \quad 8$ & $148 \quad 43 \quad 38$ & 5.00 & 2.00 & 5.0 & 1.000 & 700 & $<.5$ & N \\
\hline 422258 & 652954 & 1484936 & 7.00 & 2.00 & 5.0 & $>1.000$ & 2,000 & N & $N$ \\
\hline 422259 & 653430 & $148 \quad 16 \quad 37$ & 10.00 & 1.50 & 5.0 & 1.000 & 1,500 & $N$ & $N$ \\
\hline 422260 & 653343 & $14746 \quad 18$ & 7.00 & 2.00 & 3.0 & 1.000 & 2,000 & N & $\mathrm{N}$ \\
\hline 422261 & 654327 & $14714 \quad 0$ & 10.00 & 2.00 & 5.0 & $>1.000$ & 1,500 & N & N \\
\hline 422262 & 654346 & 1471730 & 10.00 & 2.00 & 5.0 & $>1.000$ & 3,000 & N & $N$ \\
\hline 422263 & 654318 & 1472146 & 7.00 & 1.50 & 5.0 & $>1.000$ & 2,000 & $N$ & $N$ \\
\hline 422264 & 654252 & $14726 \quad 35$ & 5.00 & 2.00 & 3.0 & 1.000 & 500 & .5 & N \\
\hline 422265 & 655945 & $149 \quad 16 \quad 59$ & 7.00 & 1.50 & 5.0 & 1.000 & 1,500 & $N$ & N \\
\hline 422266 & 655954 & $14923 \quad 52$ & 7.00 & 2.00 & 5.0 & 1.000 & 1,000 & N & $N$ \\
\hline 422267 & 655819 & 1492146 & 10.00 & 3.00 & 7.0 & 1.000 & 3,000 & $N$ & H \\
\hline 422268 & 655644 & 1492352 & 10.00 & 2.00 & 10.0 & 1.000 & 1,500 & N & $N$ \\
\hline 422269 & 65509 & 1493724 & 10.00 & 2.00 & 3.0 & $>1.000$ & 1,500 & $N$ & N \\
\hline 422270 & 65523 & 1494121 & 7.00 & 3.00 & 10.0 & 1.000 & 1,000 & N & $N$ \\
\hline 422271 & 655244 & 1493432 & 7.00 & 2.00 & 7.0 & 1.000 & 2,000 & $N$ & $\mathrm{H}$ \\
\hline 422272 & 653655 & 148119 & 5.00 & 1.50 & 3.0 & 1.000 & 500 & 6.5 & $\mathrm{~N}$ \\
\hline 422273 & 654027 & $148 \quad 1547$ & 5.00 & 1.50 & 3.0 & 1.000 & 1,000 & $N$ & $N$ \\
\hline 422274 & 65447 & 1481152 & 5.00 & 1.50 & 3.0 & .700 & 700 & N & $N$ \\
\hline 422275 & 654246 & $148 \quad 226$ & 10.00 & 2.00 & 3.0 & .700 & 1,000 & N & N \\
\hline 422276 & 654215 & $14758 \quad 6$ & 5.00 & 1.50 & 2.0 & .700 & 500 & N & N \\
\hline 422277 & $6544 \quad 12$ & 1475320 & 10.00 & 2.00 & 3.0 & .700 & 1,000 & $<.5$ & N \\
\hline 422278 & $6544 \quad 44$ & 1475020 & 3.00 & 1.50 & 2.0 & .700 & 500 & $N$ & $N$ \\
\hline 422279 & 654210 & $147 \quad 42 \quad 42$ & 7.00 & 2.00 & 2.0 & 1.000 & 1,000 & $<.5$ & N \\
\hline 422280 & $6542 \quad 2$ & 1474131 & 1.00 & .50 & 1.5 & .200 & 200 & $N$ & N \\
\hline 422281 & 654520 & 1473741 & 5.00 & 2.00 & 3.0 & 1.000 & 1,000 & $N$ & N \\
\hline 422282 & 654848 & 1472621 & 5.00 & 2.00 & 3.0 & 1.000 & 700 & $N$ & N \\
\hline 422283 & $6548 \quad 15$ & 1471745 & 7.00 & 2.00 & 2.0 & 1.000 & 700 & $N$ & N \\
\hline 422284 & 654927 & 1471158 & 5.00 & 2.00 & 3.0 & 1.000 & 2,000 & N & $H$ \\
\hline 422285 & 654913 & 1471018 & 5.00 & 1.50 & 2.0 & 1.000 & 1,000 & $N$ & $n$ \\
\hline 422286 & 654956 & $147 \quad 039$ & 10.00 & 2.00 & 2.0 & 1.000 & 2,000 & N & $N$ \\
\hline 422287 & $6545 \quad 2$ & $147 \quad 8 \quad 44$ & 5.00 & 1.50 & 2.0 & 1.000 & 1,500 & $N$ & $n$ \\
\hline 422289 & 653956 & 1473419 & 5.00 & 1.50 & 2.0 & 1.000 & 1,000 & N & N \\
\hline
\end{tabular}


TABLE 2. --RESULTS OF SPECTROgRAPHIC ANALYSES OF NURE STREAM-SEDIMENT SAMPLES FROM tHE LIVENGO0D

AND WESTERN $1 / 3$ OF THE CIRCLE QUADRANGLES, ALASKA--Continued

\begin{tabular}{|c|c|c|c|c|c|c|c|c|c|c|}
\hline Sample & $\begin{array}{c}\text { B-ppi } \\
5\end{array}$ & $\begin{array}{c}\text { Ba-ppla } \\
5\end{array}$ & $\begin{array}{c}\text { Be-ppt } \\
5\end{array}$ & $\begin{array}{l}\text { Bi-ppt } \\
5\end{array}$ & $\begin{array}{c}\text { Co-ppa } \\
5\end{array}$ & $\begin{array}{c}C r-p p r i \\
5\end{array}$ & $\begin{array}{c}\text { Cu-ppi } \\
5\end{array}$ & $\begin{array}{c}\text { La-pp五 } \\
s\end{array}$ & $\begin{array}{c}\text { Mo-pp } \\
5\end{array}$ & $\begin{array}{c}A b-p p \operatorname{m} \\
s\end{array}$ \\
\hline 422243 & 50 & 1,500 & 11.0 & $N$ & 50 & 200 & 20 & 50 & $N$ & $N$ \\
\hline 422244 & 70 & 1,500 & $\{1.0$ & $N$ & 20 & 200 & 30 & 50 & $N$ & $<20$ \\
\hline 422245 & 50 & 2,000 & $N$ & $N$ & 10 & 200 & 15 & 30 & $N$ & $<20$ \\
\hline 422247 & 70 & 2,000 & $\langle 1.0$ & $N$ & 10 & 150 & 15 & 50 & $N$ & $<20$ \\
\hline 422248 & 70 & 2,000 & 1.0 & $N$ & 20 & 500 & 30 & 50 & N & $<20$ \\
\hline 422249 & 50 & 1,500 & $\langle 1.0$ & $N$ & 10 & 200 & 15 & 50 & $N$ & $<20$ \\
\hline 422250 & 70 & 2,000 & $\langle 1.0$ & $N$ & 15 & 150 & 20 & 50 & $N$ & $<20$ \\
\hline 422251 & 70 & 1,000 & $\langle 1.0$ & $<10$ & 20 & 500 & 50 & 20 & 5 & 20 \\
\hline 422252 & 70 & 2,000 & $\langle 1.0$ & $N$ & 15 & 200 & 30 & 50 & $N$ & 20 \\
\hline 422253 & 70 & 2,000 & 01.0 & $N$ & 15 & 200 & 30 & 30 & $N$ & $\langle 20$ \\
\hline 422254 & 100 & 2,000 & $\$ 1.0$ & $N$ & 15 & 200 & 20 & 50 & $N$ & $<20$ \\
\hline 422255 & 70 & 2,000 & $\{1.0$ & $H$ & 20 & 300 & 30 & 50 & $N$ & $<20$ \\
\hline 422256 & 100 & 2,000 & 41.0 & $N$ & 15 & 200 & 30 & 50 & $N$ & 120 \\
\hline 422257 & 70 & 2,000 & $\langle 1.0$ & $N$ & 10 & 150 & 20 & 30 & $N$ & $N$ \\
\hline 422250 & 100 & 3,000 & $<1.0$ & $N$ & 15 & 300 & 50 & 50 & $N$ & $<20$ \\
\hline 422259 & 100 & 2,000 & 1.0 & $N$ & 15 & 200 & 30 & 30 & $N$ & $N$ \\
\hline 422260 & 70 & 2,000 & $\langle 1.0$ & $N$ & 20 & 200 & 20 & 30 & $N$ & 120 \\
\hline 422261 & 100 & 2,000 & 1.0 & $N$ & 15 & 300 & 20 & 50 & $N$ & 20 \\
\hline 422262 & 70 & 3,000 & $\langle 1.0$ & N & 20 & 200 & 50 & 50 & N & $N$ \\
\hline 422263 & 100 & 2,000 & 1.0 & $N$ & 15 & 300 & 30 & 50 & $N$ & $<20$ \\
\hline 422264 & 100 & 2,000 & 1.0 & $N$ & 20 & 200 & 50 & 50 & $<5$ & $N$ \\
\hline 422265 & 70 & 3,000 & 1.0 & $H$ & 20 & 300 & 30 & 50 & $N$ & $\langle 20$ \\
\hline 422266 & 70 & 2,000 & $\langle 1.0$ & $N$ & 20 & 200 & 30 & 50 & $N$ & 20 \\
\hline 422267 & 100 & 3,000 & $\langle 1.0$ & $M$ & 20 & 200 & 50 & 50 & $N$ & $N$ \\
\hline 422268 & 100 & 2,000 & 01.0 & $N$ & 20 & 200 & 50 & 30 & N & $N$ \\
\hline 422269 & 100 & 2,000 & $<1.0$ & $N$ & 20 & 300 & 30 & 50 & $N$ & $<20$ \\
\hline 422270 & 50 & 2,000 & $\langle 1.0$ & $N$ & 15 & 200 & 50 & 20 & $N$ & $\mathbb{N}$ \\
\hline 422271 & 70 & 2,000 & 1.0 & $N$ & 20 & 200 & 30 & 50 & $N$ & $N$ \\
\hline 422272 & 100 & 2,000 & 1.0 & H & 20 & 200 & 50 & 50 & $<5$ & $<20$ \\
\hline 422273 & 100 & 2,000 & 1.0 & $N$ & 15 & 150 & 20 & 50 & $N$ & $<20$ \\
\hline 422274 & 70 & 1,500 & 1.0 & $N$ & 15 & 100 & 20 & 50 & $N$ & N \\
\hline 422275 & 50 & 1,500 & $\langle 1.0$ & $N$ & 20 & 150 & 100 & $N$ & 7 & $N$ \\
\hline 422276 & 100 & 1,500 & 1.0 & $N$ & 15 & 150 & 30 & 50 & $N$ & $<20$ \\
\hline 422277 & 70 & 2,000 & 1.0 & $N$ & 20 & 150 & 50 & 50 & $N$ & $N$ \\
\hline 422278 & 100 & 2,000 & 1.0 & $N$ & 15 & 100 & 20 & 50 & $N$ & 20 \\
\hline 422279 & 100 & 2,000 & 1.0 & $N$ & 20 & 200 & 20 & 70 & $N$ & 120 \\
\hline 422280 & 30 & 700 & 1.0 & 10 & $\mathrm{~N}$ & 30 & 15 & $N$ & $N$ & $N$ \\
\hline 422281 & 100 & 2,000 & $\langle 1.0$ & $N$ & 15 & 150 & 20 & 50 & $N$ & $<20$ \\
\hline 422282 & 70 & 2,000 & $\langle 1.0$ & $H$ & 15 & 150 & 20 & 50 & N & $<20$ \\
\hline 422283 & 100 & 2,000 & 1.0 & $H$ & 20 & 150 & 50 & 50 & $N$ & $<20$ \\
\hline 422284 & 100 & 2,000 & 1.0 & $N$ & 20 & 100 & 30 & 50 & N & $<20$ \\
\hline 422285 & 100 & 1,500 & 1.0 & $\mathrm{H}$ & 15 & 150 & 30 & 50 & $N$ & 20 \\
\hline 422286 & 100 & 2,000 & 1.0 & $N$ & 30 & 150 & 50 & 50 & N & 20 \\
\hline 422287 & 100 & 1,500 & 1.0 & $N$ & 20 & 150 & 20 & 70 & $N$ & $<20$ \\
\hline 422288 & 100 & 1,500 & 1.0 & $N$ & 20 & 100 & 15 & 50 & H & 20 \\
\hline
\end{tabular}


TABLE 2.--RESULLTS OF SPECTROGRAPHIC ANALYSES OF NURE STREAM-SEDIMENT SAMPLES FROM THE LIVENGDOD

AND WESTERN $1 / 3$ OF THE CIRCLE QUADRANGLES, ALASKA--Continued

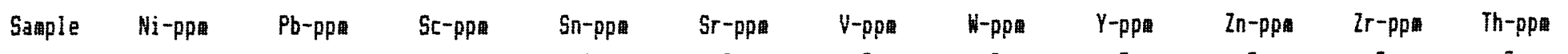

$\begin{array}{lllllllll}5 & 5 & 5 & 5 & 5 & s & 5 & 5 & 5\end{array}$

\begin{tabular}{|c|c|c|c|c|c|c|c|c|c|c|}
\hline 422243 & 50 & 15 & 20 & N & 150 & 150 & $N$ & 30 & $N$ & 200 \\
\hline 422244 & 50 & 20 & 20 & $N$ & 200 & 150 & $N$ & 50 & $N$ & 500 \\
\hline 422245 & 20 & 20 & 20 & $N$ & 500 & 150 & $N$ & 20 & $N$ & 500 \\
\hline 422247 & 30 & 20 & 20 & $N$ & 300 & 100 & $N$ & 50 & $\mathrm{~N}$ & 500 \\
\hline 422248 & 70 & 20 & 20 & $N$ & 300 & 200 & $N$ & 50 & $N$ & 300 \\
\hline 422249 & 20 & 15 & 15 & $N$ & 200 & 150 & $N$ & 30 & $N$ & 200 \\
\hline 422250 & 30 & 20 & 20 & $N$ & 300 & 150 & $N$ & 50 & $N$ & 200 \\
\hline 422251 & 70 & 30 & 20 & $N$ & 150 & 150 & $N$ & 20 & $N$ & 150 \\
\hline 422252 & 30 & 20 & 20 & $N$ & 500 & 200 & $N$ & 50 & $N$ & 500 \\
\hline 422253 & 30 & 20 & 20 & $N$ & 500 & 200 & $N$ & 30 & $N$ & 500 \\
\hline 422254 & 30 & 20 & 20 & N & 500 & 150 & $N$ & 50 & $N$ & 700 \\
\hline 422255 & 50 & 20 & 20 & $N$ & 300 & 200 & $N$ & 50 & $\mathbb{N}$ & 700 \\
\hline 422256 & 50 & 20 & 20 & $N$ & 500 & 200 & $N$ & 50 & $N$ & 500 \\
\hline 422257 & 30 & 20 & 15 & N & 300 & 150 & $N$ & 20 & $N$ & 300 \\
\hline 422258 & 30 & 20 & 30 & N & 500 & 200 & $N$ & 50 & $N$ & 1,000 \\
\hline 422259 & 50 & 20 & 20 & N & 500 & 200 & $N$ & 50 & $\mathrm{~N}$ & 500 \\
\hline 422260 & 50 & 20 & 20 & $N$ & 300 & 200 & $\mathbb{N}$ & 50 & $\mathrm{~N}$ & 1,000 \\
\hline 422261 & 30 & 20 & 30 & $N$ & 300 & 200 & $N$ & 50 & $N$ & 1,000 \\
\hline 422262 & 30 & 15 & 20 & N & 500 & 200 & $N$ & 50 & N & 1,000 \\
\hline 422263 & 50 & 15 & 20 & $N$ & 500 & 200 & $N$ & 50 & $N$ & 1,000 \\
\hline 422264 & 50 & 30 & 20 & $N$ & 200 & 300 & $N$ & 30 & $N$ & 200 \\
\hline 422265 & 50 & 20 & 20 & $<10$ & 500 & 200 & $N$ & 50 & $\mathrm{~N}$ & 700 \\
\hline 422266 & 50 & 30 & 20 & $N$ & 500 & 200 & $N$ & 50 & $N$ & 300 \\
\hline 422267 & 50 & 20 & 20 & $N$ & 500 & 200 & $\mathrm{~N}$ & 50 & $\mathrm{~N}$ & 300 \\
\hline 422268 & 50 & 20 & 20 & N & 500 & 200 & N & 50 & $N$ & 300 \\
\hline
\end{tabular}

$422269 \quad 50 \quad 30 \quad 30$

$422270 \quad 30 \quad 10 \quad 20$

$\begin{array}{lll}N & 500 & 200 \\ H & 500 & 200\end{array}$

$422271 \quad 50 \quad 15 \quad 30$

$422272 \quad 50 \quad 20 \quad 20$

$\begin{array}{llll}422273 & 50 & 20 & 20\end{array}$

<10 $500 \quad 200$

$300 \quad 150$

$300 \quad 150$

$\begin{array}{ll}422274 & 30 \\ 422275 & 50 \\ 422276 & 50 \\ 422277 & 3 \\ 422278 & 30\end{array}$

$30 \quad 15 \quad 15$

$300 \quad 150$

$300 \quad 150$

$200 \quad 150$

$300 \quad 200$

$\begin{array}{llll}422279 & 30 & 15 & 15\end{array}$

$300 \quad 100$

$\begin{array}{ll}N & 50 \\ N & 30 \\ N & 50 \\ N & 50 \\ N & 30\end{array}$

$\begin{array}{ll}N & 700 \\ N & 200 \\ N & 300 \\ N & 150 \\ N & 300\end{array}$

$\begin{array}{llll}42279 & 50 & 50 & 20\end{array}$

$\begin{array}{rrrr}422280 & 10 & 10 & 7 \\ 422281 & 30 & 20 & 20\end{array}$

$\begin{array}{llll}422282 & 30 & 30 & 20\end{array}$

$\begin{array}{llll}422283 & 50 & 30 & 20\end{array}$

$422284 \quad 50$

$422285-50$

$422286 \quad 70 \quad 50 \quad 30$

$422287 \quad 30$

$20 \quad 20$

$\begin{array}{rr}300 & 150 \\ 1100 & 70 \\ 300 & 150 \\ 300 & 150 \\ 200 & 200\end{array}$

$\begin{array}{ll}N & 30 \\ N & 30 \\ N & 30 \\ N & 50 \\ N & 50\end{array}$

$\begin{array}{ll}N & 200 \\ N & 150 \\ N & 200 \\ N & 300 \\ N & 200\end{array}$

$422288 \quad 30$

20

20

$\begin{array}{rrr}N & 300 & 150 \\ N & 4100 & 70 \\ N & 300 & 150 \\ N & 300 & 150 \\ N & 200 & 200\end{array}$

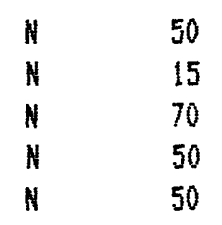

$300 \quad 150$

$300 \quad 150$

$200 \quad 200$

$300 \quad 150$

$300 \quad 150$ 
TABLE 2.--RESULTS OF SPECTROGRAPHIC ANALYSES OF NURE STREAM-SEDIMENT SAMPLES FROM THE LIVENGOOD AND WESTERN $1 / 3$ OF THE CIRCLE QUADRANGLES, ALASKA--ContinUEd

\begin{tabular}{|c|c|c|c|c|c|c|c|c|c|}
\hline Sa解le & Latitude & Longi tude & $\begin{array}{c}\text { Fe-pct. } \\
s\end{array}$ & $\begin{array}{c}\text { Mq-pct. } \\
s\end{array}$ & $\underset{s}{C a-p c t .}$ & $\begin{array}{c}\text { Ti-pct. } \\
s\end{array}$ & $\begin{array}{c}\text { Mn-ppi } \\
5\end{array}$ & $\begin{array}{c}\text { Ag-ppi } \\
5\end{array}$ & $\begin{array}{c}\text { As-pp } \\
5\end{array}$ \\
\hline 422289 & 653849 & 1474910 & 5.00 & 1.50 & 2.0 & 1.000 & 1,000 & N & N \\
\hline 422290 & 653946 & 1475526 & 5.00 & 2.00 & 3.0 & 1.000 & 1,000 & $n$ & $N$ \\
\hline 422291 & 653533 & 1481951 & 5.00 & 1.50 & 2.0 & .700 & 1,000 & N & $N$ \\
\hline 422292 & 653745 & 1482533 & 7.00 & 2,00 & 3.0 & 1.000 & 1,000 & 5.0 & $N$ \\
\hline 422293 & 653933 & $148 \quad 26 \quad 7$ & 7.00 & 2.00 & 3.0 & .700 & 2,000 & $<.5$ & N \\
\hline 422294 & 655133 & 1482455 & 5.00 & 1.50 & 3.0 & 1.000 & 700 & $H$ & N \\
\hline 422295 & 654752 & 1483141 & 5.00 & 2.00 & 3.0 & 1.000 & 700 & $N$ & N \\
\hline 422296 & 654548 & 1483338 & 5.00 & 1.50 & 2.0 & 1.000 & 500 & $N$ & $N$ \\
\hline 422297 & 653924 & 1483230 & 7.00 & 1.50 & 3.0 & 1.000 & 1,500 & N & N \\
\hline 422298 & 653933 & $14822 \quad 27$ & 5.00 & 1.50 & 2.0 & 1.000 & 500 & 30.0 & $N$ \\
\hline 422299 & 654654 & $148 \quad 749$ & 10.00 & 2.00 & 2.0 & 1.000 & 3,000 & $H$ & N \\
\hline 422300 & 654811 & 148418 & 5.00 & 1.50 & 2.0 & .700 & 700 & N & $N$ \\
\hline 422301 & 65508 & $148 \quad 2 \quad 8$ & 5.00 & 2.00 & 2.0 & 1.000 & 500 & N & $N$ \\
\hline 422302 & 655041 & $148 \quad 435$ & 5.00 & 1.50 & 1.0 & .700 & 1,500 & $N$ & N \\
\hline 422303 & 655256 & $148 \quad 245$ & 7.00 & 1.50 & 2.0 & 1.000 & 1,000 & $N$ & N \\
\hline 422304 & 653437 & 1483351 & 1.50 & 1.00 & 20.0 & .070 & 3,000 & $n$ & $N$ \\
\hline 422305 & 654336 & $148 \quad 28 \quad 0$ & 5.00 & 1.50 & 2.0 & .500 & 500 & $<.5$ & $N$ \\
\hline 422306 & 65472 & $148 \quad 1535$ & 10.00 & 2.00 & 3.0 & 1.000 & 2,000 & $N$ & $N$ \\
\hline 422307 & 65473 & $148 \quad 14 \quad 18$ & 7.00 & 2.00 & 2.0 & 1.000 & 1,000 & N & N \\
\hline 422308 & 655348 & 1483820 & 10.00 & 2.00 & 2.0 & 1.000 & 2,000 & $N$ & $N$ \\
\hline 422309 & 65447 & $14759 \quad 9$ & 7.00 & 2.00 & 3.0 & 1.000 & 2,000 & $N$ & N \\
\hline 422310 & 654542 & 1475352 & 5.00 & 1.50 & 2.0 & 1.000 & 1,500 & H & N \\
\hline 422311 & 654753 & 1475031 & 5.00 & 1.50 & 3.0 & .700 & 700 & $N$ & N \\
\hline 422312 & 655215 & 1474229 & 5.00 & 1.00 & 1.0 & .500 & 300 & H & N \\
\hline 422314 & $6556 \quad 35$ & $14749 \quad 6$ & 5.00 & 1.50 & 3.0 & 1.000 & 700 & $N$ & N \\
\hline 422315 & 6600 & 1474944 & 5.00 & 1.50 & 3.0 & .700 & 1,000 & $N$ & N \\
\hline 422316 & 65550 & 149306 & 5.00 & 1.50 & 3.0 & .700 & 1,000 & $N$ & H \\
\hline 422317 & 655811 & 1492930 & 5.00 & 1.50 & 3.0 & 1.000 & 500 & $<.5$ & N \\
\hline 422318 & 655952 & 1492948 & 3.00 & 1.50 & 3.0 & .500 & 300 & $N$ & $\mathrm{H}$ \\
\hline 422319 & 655913 & 1493333 & 3.00 & 1.50 & 3.0 & 1.000 & 500 & N & $N$ \\
\hline 422320 & 655953 & $14936 \quad 1$ & 7.00 & 2.00 & 5.0 & 1.000 & 500 & $<.5$ & N \\
\hline $42232 !$ & 655612 & $14942 \quad 28$ & 3.00 & 1.50 & 2.0 & .700 & 300 & $N$ & N \\
\hline 422322 & 655631 & 1493848 & 3.00 & 1.50 & 3.0 & .500 & 700 & $N$ & N \\
\hline 422323 & 655441 & $149 \quad 40 \quad 0$ & 3.00 & 1.50 & 3.0 & .700 & 300 & 6.5 & N \\
\hline 422324 & $6553 \quad 8$ & 1494344 & 7.00 & 2.00 & 5.0 & 1.000 & 1,000 & $N$ & $N$ \\
\hline 422325 & 654250 & $148 \quad 2054$ & 5.00 & 1.50 & 3.0 & 1.000 & 500 & $<.5$ & $N$ \\
\hline 422326 & 654443 & $148 \quad 718$ & 5.00 & 1.00 & 3.0 & .500 & 300 & $H$ & H \\
\hline 422327 & $6544 \quad 12$ & $148 \quad 444$ & 5.00 & 1.50 & 3.0 & 1.000 & 500 & N & $N$ \\
\hline 422328 & $6543 \quad 14$ & 147419 & 10.00 & 2.00 & 5.0 & 1.000 & 1,000 & $N$ & N \\
\hline 422329 & 654736 & 1473234 & 7.00 & 1.50 & 3.0 & 1.000 & 700 & H & N \\
\hline 422330 & 654731 & 1473029 & 7.00 & 1.50 & 2.0 & 1.000 & 500 & $N$ & N \\
\hline 422331 & 654829 & $147232 b$ & 7.00 & 1.50 & 2.0 & .700 & 500 & $N$ & $N$ \\
\hline 422332 & 654830 & 147142 & 5.00 & 1.50 & 2.0 & 1.000 & 700 & $N$ & N \\
\hline 422333 & 65448 & $147 \quad 139$ & 5.00 & 1.50 & 3.0 & 1.000 & 1,000 & $N$ & $N$ \\
\hline 422334 & 654443 & $147 \quad 5 \quad 8$ & 5.00 & 1.50 & 3.0 & .700 & 700 & $N$ & $N$ \\
\hline
\end{tabular}


TABLE 2:--RESULTS OF SPECTROGRAPHIC ANALYSES OF NURE STREAM-SEDIMENT SAMPLES FROM THE LIVENGOOD AND WESTERH $1 / 3$ OF THE CIRCLE QUADRANGLES, ALASKA--Continued

\begin{tabular}{|c|c|c|c|c|c|c|c|c|c|c|}
\hline Saaple & $\begin{array}{c}\text { B-ppa } \\
5\end{array}$ & $\begin{array}{c}\text { Ba-ppm } \\
5\end{array}$ & $\begin{array}{c}\text { Be-ppa } \\
5\end{array}$ & $\begin{array}{c}\text { Bi-pp } \\
5\end{array}$ & $\begin{array}{c}\text { Co-ppi } \\
5\end{array}$ & $\underset{5}{C r-p p i}$ & $\begin{array}{c}\text { Cu-ppu } \\
5\end{array}$ & $\begin{array}{l}\text { La-ppi } \\
5\end{array}$ & $\begin{array}{c}\text { Mo-ppon } \\
5\end{array}$ & $\begin{array}{c}\text { Nb-ppw } \\
5\end{array}$ \\
\hline 422289 & 100 & 1,500 & 1.0 & $H$ & 20 & 100 & 20 & 50 & $N$ & 20 \\
\hline 422290 & 70 & 2,000 & 1.0 & $N$ & 15 & 150 & 20 & 50 & N & $<20$ \\
\hline 422291 & 70 & 1,500 & 1.0 & $N$ & 20 & 100 & 20 & 50 & $N$ & $<20$ \\
\hline 422292 & 100 & 2,000 & 1.0 & $N$ & 20 & 150 & 20 & 50 & $N$ & 20 \\
\hline 422293 & 70 & 1,500 & 1.0 & $H$ & 30 & 150 & 30 & 30 & N & $N$ \\
\hline 422294 & 70 & 2,000 & 1.0 & $N$ & 15 & 100 & 15 & 30 & $N$ & $<20$ \\
\hline 422295 & 100 & 2,000 & 1.0 & $N$ & 20 & 200 & 20 & 50 & $H$ & 20 \\
\hline 422296 & 70 & 1,000 & 1.0 & $N$ & 15 & 100 & 15 & 30 & $N$ & $N$ \\
\hline 422297 & 100 & 1,500 & 1.0 & $N$ & 15 & 150 & 20 & 50 & $N$ & $<20$ \\
\hline 422298 & 100 & 1,500 & 1.0 & $N$ & 15 & 100 & 20 & 50 & $N$ & $<20$ \\
\hline 422299 & 70 & 2,000 & 1.0 & $H$ & 15 & 100 & 20 & 50 & $N$ & $N$ \\
\hline 422300 & 70 & 1,500 & 1.0 & $N$ & 15 & 100 & 20 & 50 & N & $H$ \\
\hline 422301 & 100 & 2,000 & 1.0 & $N$ & 20 & 150 & 30 & 70 & $N$ & 30 \\
\hline 422302 & 100 & 1,500 & 1.5 & $N$ & 15 & 100 & 20 & 30 & $\langle 5$ & 20 \\
\hline 422303 & 100 & 1,500 & 1.0 & $H$ & 20 & 150 & 50 & 50 & 7 & 20 \\
\hline 422304 & 20 & 500 & $\langle 1.0$ & H & 7 & 30 & 15 & $N$ & $N$ & $N$ \\
\hline 422305 & 100 & 1,000 & 1.0 & $\mathrm{~N}$ & 15 & 70 & 30 & 30 & $<5$ & $\mathrm{~N}$ \\
\hline 422306 & 100 & 2,000 & 1.0 & $N$ & 20 & 100 & 50 & 50 & $\langle 5$ & 30 \\
\hline 422307 & 100 & 2,000 & 1.0 & $N$ & 30 & 200 & 50 & 50 & $N$ & $(20$ \\
\hline 422308 & 100 & 2,000 & 1.0 & N & 30 & 200 & 30 & 30 & 5 & $<20$ \\
\hline 422309 & 70 & 1,000 & 1.0 & $N$ & 20 & 1,000 & 50 & 50 & $N$ & $N$ \\
\hline 422310 & 100 & 1,500 & $\langle 1.0$ & $N$ & 20 & 100 & 30 & 50 & $N$ & $<20$ \\
\hline 422311 & 70 & 1,500 & $\langle 1.0$ & $N$ & 15 & 100 & 20 & 30 & H & $N$ \\
\hline 422312 & 50 & 1,000 & 1.0 & $N$ & 10 & 50 & 20 & 30 & $<5$ & $H$ \\
\hline 422314 & 70 & 1,500 & 1.0 & $N$ & 15 & 100 & 15 & 50 & $N$ & 20 \\
\hline 422315 & 50 & 1,500 & $\langle 1.0$ & $N$ & 15 & 100 & 50 & 30 & $N$ & $\langle 20$ \\
\hline 422316 & 70 & 2,000 & 1.0 & $N$ & 10 & 150 & 15 & 50 & $N$ & $N$ \\
\hline 422317 & 70 & 2,000 & 1.0 & $\mathbf{N}$ & 15 & 150 & 30 & 30 & $N$ & $<20$ \\
\hline 422318 & 50 & 1,000 & 1.0 & $N$ & 10 & 70 & 15 & $N$ & $<5$ & $N$ \\
\hline 422319 & 70 & 2,000 & 1.0 & $N$ & 10 & 150 & 20 & 50 & $N$ & $<20$ \\
\hline 422320 & 70 & 2,000 & 1.0 & $N$ & 15 & 150 & 50 & 50 & $<5$ & $<20$ \\
\hline 422321 & 70 & 700 & 1.0 & $N$ & 10 & 70 & 15 & 30 & $N$ & $N$ \\
\hline 422322 & 50 & 700 & 1.0 & $N$ & 10 & 50 & 15 & $N$ & $N$ & $H$ \\
\hline 422323 & 70 & 2,000 & 1.0 & $H$ & 10 & 100 & 50 & 50 & $N$ & $N$ \\
\hline 422324 & 70 & 2,000 & 1.0 & $N$ & 20 & 150 & 50 & 50 & $N$ & $N$ \\
\hline 422325 & 70 & 2,000 & 1.0 & $N$ & 15 & 150 & 50 & 50 & 7 & $N$ \\
\hline 422326 & 30 & 700 & 1.0 & $N$ & 10 & 50 & 15 & $N$ & 45 & $N$ \\
\hline 422327 & 70 & 1,500 & 1.0 & $N$ & 15 & 100 & 30 & 50 & $<5$ & $N$ \\
\hline 422328 & 100 & 2,000 & 1.0 & $N$ & 20 & 200 & 50 & 70 & $N$ & $N$ \\
\hline 422329 & 70 & 2,000 & 1.0 & $N$ & 15 & 150 & 20 & 50 & $N$ & $N$ \\
\hline 422330 & 100 & 2,000 & 1.0 & $N$ & 20 & 150 & 50 & 50 & $N$ & 20 \\
\hline 422331 & 70 & 2,000 & 1.0 & $N$ & 15 & 150 & 20 & 50 & $N$ & $<20$ \\
\hline 422332 & 70 & 1,500 & 1.0 & $N$ & 15 & 100 & 20 & 30 & $N$ & 20 \\
\hline 422333 & 70 & 1,500 & 1.0 & $N$ & 15 & 150 & 20 & 50 & $N$ & $N$ \\
\hline 422334 & 70 & 1,500 & 1.0 & $N$ & 10 & 150 & 15 & 20 & $N$ & $N$ \\
\hline
\end{tabular}


TABLE 2.--RESUL TS OF SPECTROGRAFHIC AMALYSES OF NURE STREAM-SEDIMENT SAMPLES FROM THE LIVENGOOD

AND WESTERN $1 / 3$ OF THE CIRCLE QUADRANGLES, ALASKA--ContinUEd

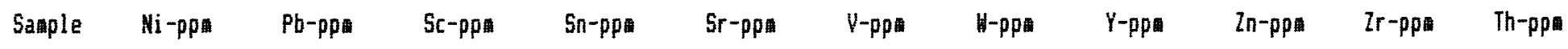

$5 \quad s \quad 5 \quad 5$

క

5

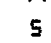

s

5

5

5

\begin{tabular}{|c|c|c|c|c|c|c|c|c|c|c|}
\hline 422289 & 50 & 20 & 20 & $N$ & 200 & 150 & $N$ & 50 & $N$ & 300 \\
\hline 422290 & 30 & 20 & 20 & $N$ & 300 & 200 & $N$ & 50 & $N$ & 500 \\
\hline 422291 & 50 & 20 & 20 & $N$ & 300 & 150 & $N$ & 50 & 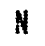 & 200 \\
\hline 422292 & 30 & 50 & 20 & N & 500 & 200 & $\mathrm{H}$ & 50 & $N$ & 300 \\
\hline 422293 & 50 & 30 & 20 & N & 300 & 150 & $N$ & 30 & $N$ & 150 \\
\hline 422294 & 30 & 20 & 20 & $N$ & 300 & 150 & $\mathbb{N}$ & 30 & $N$ & 500 \\
\hline 422295 & 30 & 20 & 20 & N & 300 & 200 & $N$ & 50 & $N$ & 700 \\
\hline 422296 & 30 & 15 & 20 & $N$ & 200 & 150 & $N$ & 50 & $N$ & 200 \\
\hline 422297 & 30 & 30 & 20 & $N$ & 300 & 150 & $\mathbf{N}$ & 50 & N & 500 \\
\hline 422298 & 30 & 20 & 15 & $N$ & 200 & 150 & $N$ & 50 & $N$ & 300 \\
\hline 422299 & 50 & 20 & 20 & $N$ & $<100$ & 200 & $N$ & 30 & $N$ & 150 \\
\hline 422300 & 30 & 15 & 20 & $N$ & 200 & 150 & $N$ & 50 & $\mathbb{N}$ & 200 \\
\hline 422301 & 30 & 30 & 20 & $N$ & 200 & 200 & $N$ & 70 & $N$ & 700 \\
\hline 422302 & 50 & 20 & 15 & 30 & 100 & 150 & $N$ & 50 & $N$ & 500 \\
\hline 422303 & 50 & 70 & 20 & 50 & 200 & 150 & $N$ & 70 & $N$ & 500 \\
\hline 422304 & 10 & 10 & 10 & $N$ & 300 & 30 & $N$ & 10 & $N$ & 50 \\
\hline 422305 & 30 & 20 & 15 & $N$ & 100 & 150 & $N$ & 30 & $\mathbf{N}$ & 200 \\
\hline 422306 & 50 & 30 & 30 & 10 & 200 & 200 & $N$ & 70 & $N$ & 500 \\
\hline 422307 & 50 & 50 & 20 & N & 200 & 200 & 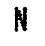 & 50 & $N$ & 500 \\
\hline 422308 & 50 & 30 & 30 & 20 & 200 & 300 & $N$ & 50 & $N$ & 300 \\
\hline 422309 & 100 & 30 & 30 & $N$ & 200 & 200 & $N$ & 50 & $N$ & 300 \\
\hline 422310 & 30 & 20 & 20 & $N$ & 200 & 150 & $N$ & 50 & $\mathbf{N}$ & 300 \\
\hline 422311 & 30 & 15 & 15 & N & 200 & 150 & $N$ & 30 & $\mathbf{N}$ & 200 \\
\hline 422312 & 30 & 20 & 15 & $N$ & 150 & 150 & $N$ & 30 & $\mathbb{N}$ & 200 \\
\hline 422314 & 30 & 20 & 20 & $N$ & 300 & 150 & $N$ & 50 & $N$ & 300 \\
\hline
\end{tabular}

$\begin{array}{ll}422315 & 5 \\ 422316 & 30 \\ 422317 & 50 \\ 422318 & 30 \\ 422319 & 30\end{array}$

$$
15 \quad 20
$$

N $\quad 300 \quad 200$

50
30

$\begin{array}{ll}15 & 20 \\ 15 & 20\end{array}$

300

100

$500 \quad 150$

$20 \quad 20$

20

200

100

$20 \quad 20$

300

150

$300 \quad 200$

$422320 \quad 50$

$20 \quad 20$

110

$\begin{array}{ll}422321 & 30 \\ 422322 & 30\end{array}$

$10 \quad 15$

$10 \quad 15$

422323

$20 \quad 20$

$422324 \quad 30$

$20 \quad 20$

150

150

100

$300 \quad 150$

$300 \quad 200$

$\begin{array}{ll}N & 50 \\ N & 50 \\ N & 50 \\ N & 30 \\ N & 50\end{array}$

$\begin{array}{ll}N & 200 \\ N & 300 \\ N & 200 \\ N & 150 \\ N & 500\end{array}$

$\begin{array}{llll}422325 & 50 & 20 & 20\end{array}$

$42232 \mathrm{~b} \quad 30$

$15 \quad 10$

422327

422328

30

$20 \quad 20$

$20 \quad 20$

$422329 \quad 50$
20
20

422330

$\begin{array}{lll}50 & 20 & 20 \\ 30 & 15 & 20 \\ 30 & 20 & 15 \\ 30 & 20 & 15 \\ 30 & 15 & 15\end{array}$

$\begin{array}{lll}50 & 20 & 20 \\ 30 & 15 & 20 \\ 30 & 20 & 15 \\ 30 & 20 & 15 \\ 30 & 15 & 15\end{array}$

422331

422332

422334

$\begin{array}{rll}100 & 300 & 200 \\ N & 100 & 100 \\ N & 300 & 150 \\ \langle 10 & 500 & 200 \\ N & 500 & 150\end{array}$

$\begin{array}{ll}N & 50 \\ N & 30 \\ N & 20 \\ N & 30 \\ N & 50\end{array}$

$\begin{array}{ll}N & 200 \\ N & 150 \\ N & 100 \\ N & 200 \\ N & 200\end{array}$

$\begin{array}{ll}N & 50 \\ N & 30 \\ N & 50 \\ N & 70 \\ N & 50\end{array}$

$\begin{array}{ll}N & 200 \\ N & 150 \\ N & 300 \\ N & 200 \\ N & 300\end{array}$

$300 \quad 150$

$300 \quad 200$

150

150

$\begin{array}{ll}300 & 150 \\ 300 & 100\end{array}$

$\begin{array}{ll}N & 30 \\ N & 30 \\ N & 50 \\ N & 50 \\ N & 30\end{array}$

$\begin{array}{ll}N & 200 \\ N & 200 \\ N & 300 \\ N & 300 \\ N & 300\end{array}$


TABLE 2.--RESULTS OF SPECTRDGRAPHIC ANALYSES OF MURE STREAM-SEDIMENT SAMPLES FRON THE LIVENGDOD AHD WESTERN $1 / 3$ OF THE CIRCLE QUADRANGLES, ALASKA--Continued

\begin{tabular}{|c|c|c|c|c|c|c|c|c|c|}
\hline Sample & Latitude & Longitude & $\begin{array}{c}\text { Fe-pet. } \\
5\end{array}$ & $\begin{array}{c}\text { Mg-pct. } \\
5\end{array}$ & $\begin{array}{c}\text { Ca-pet. } \\
5\end{array}$ & $\begin{array}{c}\text { Ti-pct. } \\
5\end{array}$ & $\begin{array}{c}M n-p p M \\
5\end{array}$ & $\begin{array}{c}A g-p p \\
5\end{array}$ & $\begin{array}{c}\text { As-ppa } \\
5\end{array}$ \\
\hline 422335 & 654344 & 1472249 & 5.00 & 1.50 & 3.0 & .700 & 1,000 & N & H \\
\hline 422336 & 653526 & 1482351 & 7.00 & 2.00 & 3.0 & 31.000 & 1,000 & $N$ & $H$ \\
\hline 422337 & 654349 & $148 \quad 26 \quad 15$ & 5.00 & 1.50 & 3.0 & 1.000 & 1,000 & $N$ & $N$ \\
\hline 422338 & 655657 & $14823 \quad 33$ & 5.00 & 1.50 & 3.0 & 1.000 & 1,000 & $N$ & N \\
\hline 422339 & $6558 \quad 5$ & $14824 \quad 15$ & .20 & .15 & 2.0 & .050 & 300 & $N$ & N \\
\hline 422340 & 655935 & 1482241 & 7.00 & 2.00 & 3.0 & 1.000 & 700 & $N$ & $N$ \\
\hline 422342 & 655949 & 1483046 & 5.00 & 1.50 & 3.0 & 1.000 & 500 & $H$ & $N$ \\
\hline 422344 & 655851 & 1483231 & 7.00 & 2.00 & 3.0 & 1.000 & 1,000 & $N$ & H \\
\hline 422345 & 655733 & 148323 & 1.00 & .50 & 3.0 & .300 & 300 & H & $N$ \\
\hline 422346 & 655559 & 1483024 & 7.00 & 1.50 & 3.0 & 1.000 & 2,000 & $<.5$ & H \\
\hline 422347 & $6554 \quad 1$ & $14831 \quad 12$ & 10.00 & 2.00 & 1.0 & .700 & 2,000 & $N$ & H \\
\hline 422348 & 655129 & 1483159 & 10.00 & 2.00 & 3.0 & 1.000 & 2,000 & $N$ & $N$ \\
\hline 422352 & 654348 & 1483033 & 5.00 & 1.50 & 3.0 & .700 & 500 & $N$ & $N$ \\
\hline 422353 & 653934 & $148 \quad 2039$ & 10.00 & 1.50 & 3.0 & .700 & 700 & $N$ & H \\
\hline 422354 & 655456 & $148 \quad 830$ & 7.00 & 1.50 & 3.0 & 1.000 & 500 & N & $N$ \\
\hline 422355 & 655747 & $148 \quad 450$ & 5.00 & 1.50 & 2.0 & .700 & 500 & $<.5$ & H \\
\hline 422356 & 655913 & $148 \quad 252$ & 5.00 & 1.50 & 3.0 & 1.000 & 500 & $N$ & $N$ \\
\hline 422357 & $6558 \quad 0$ & 1475640 & 10.00 & 3.00 & 3.0 & 1.000 & 2,000 & $N$ & $H$ \\
\hline 422358 & 653730 & 1483345 & 1.00 & .20 & 3.0 & .100 & 500 & N & H \\
\hline 422359 & 655045 & 1482129 & 7.00 & 1.50 & 2.0 & .700 & 700 & $N$ & $N$ \\
\hline 422360 & 65523 & $14927 \quad 5$ & 10.00 & 1.50 & 3.0 & .700 & 700 & $<.5$ & N \\
\hline 422363 & 655616 & $148 \quad 17 \quad 32$ & 5.00 & 1.50 & 3.0 & 1.000 & 500 & $N$ & N \\
\hline 422364 & 655541 & 1491435 & 7.00 & 1.50 & 2.0 & 1.000 & 1,000 & $N$ & $N$ \\
\hline 422365 & 655840 & 1482025 & 10.00 & 1.50 & 2.0 & 1.000 & 1,500 & $N$ & $N$ \\
\hline 422366 & 655817 & 1483647 & 10.00 & 2.00 & 3.0 & 1.000 & 1,500 & $N$ & $N$ \\
\hline 422367 & 654918 & $14840 \quad 44$ & 7.00 & 1.50 & 3.0 & 1.000 & 700 & N & N \\
\hline 422368 & $6548 \quad 8$ & $14838 \quad 20$ & 7.00 & 1.50 & 2.0 & .700 & 1,000 & $H$ & $H$ \\
\hline 422369 & 65452 & $148 \quad 3655$ & 7.00 & 1.50 & 3.0 & 1.000 & 1,000 & N & N \\
\hline 422370 & 653541 & 1483656 & 5.00 & 1.50 & 3.0 & .700 & 500 & $N$ & $N$ \\
\hline 422371 & 655818 & 1485013 & 5.00 & 2.00 & 3.0 & 1.000 & 700 & $H$ & N \\
\hline 422372 & 65598 & 1474958 & 3.00 & 1.50 & 2.0 & .700 & 500 & N & $N$ \\
\hline 422373 & 655929 & 1475026 & 7.00 & 1.50 & 1.5 & .700 & 1,500 & $N$ & $N$ \\
\hline 422374 & 655951 & 1474318 & 5.00 & 1.50 & 5.0 & .700 & 500 & $H$ & $H$ \\
\hline 422375 & 655956 & 1473938 & 3.00 & 1.50 & 3.0 & .500 & 500 & $N$ & $N$ \\
\hline 422376 & 655621 & 1471946 & 1.00 & .20 & .7 & .100 & 150 & N & H \\
\hline 422377 & 654624 & 1474336 & 2,00 & .70 & 1.0 & .200 & 200 & <.5 & $H$ \\
\hline 422379 & 653350 & 1484157 & 3.00 & 1.50 & 2.0 & .700 & 300 & $N$ & $H$ \\
\hline 422379 & 653423 & 1484136 & 3.00 & 1.50 & 1.5 & .500 & 200 & $N$ & N \\
\hline 422380 & 653655 & 1485357 & 5.00 & 2.00 & 2.0 & .700 & 700 & N & $N$ \\
\hline 422381 & 653612 & 1485353 & 3.00 & 1.50 & 1.5 & .500 & 500 & N & $N$ \\
\hline 422382 & 653951 & 1485922 & 2.00 & .70 & 1.0 & .500 & 200 & $H$ & $N$ \\
\hline 422383 & 654435 & $14848 \quad 0$ & 7.00 & 2.00 & 1.5 & .500 & 700 & $N$ & $N$ \\
\hline 422384 & 655135 & $149 \quad 46 \quad 17$ & 7.00 & 2.00 & 2.0 & .500 & 1,000 & $<.5$ & $N$ \\
\hline 422385 & 655054 & 1495423 & 2.00 & .70 & .7 & .300 & 200 & $N$ & $N$ \\
\hline 422387 & 655956 & $14736 \quad 37$ & 3.00 & 1.50 & 1.0 & .700 & 300 & $N$ & H \\
\hline
\end{tabular}


TABLE 2.--RESULTS OF SPECTROGRAPHIC ANALYSES OF NURE STREAM-SEDIMENT SAMPLES FROM THE LIVENGDOD

AND HESTERN $1 / 3$ OF THE CIRCLE QUADRAMGLES, ALASKA--Continued

\begin{tabular}{|c|c|c|c|c|c|c|c|c|c|c|}
\hline Sample & $\begin{array}{c}\text { B-pp } \\
5\end{array}$ & $\begin{array}{c}\text { Ba-pp } \\
5\end{array}$ & $\begin{array}{c}\text { Be-ppo } \\
5\end{array}$ & $\begin{array}{c}\text { Bi-ppm } \\
5\end{array}$ & $\begin{array}{c}\text { Co-pp } \\
5\end{array}$ & $\begin{array}{c}{[r-p p \text { i }} \\
5\end{array}$ & $\begin{array}{c}\text { Cu-pp } \\
5\end{array}$ & $\begin{array}{l}\text { La-ppw } \\
5\end{array}$ & $\begin{array}{c}\text { Mo-ppo } \\
5\end{array}$ & $\begin{array}{c}\text { Nb-ppo } \\
5\end{array}$ \\
\hline 422335 & 70 & 1,500 & 1.0 & $N$ & 10 & 100 & 15 & $N$ & $N$ & $N$ \\
\hline 422336 & 100 & 2,000 & $\langle 1.0$ & $N$ & 15 & 200 & 30 & 70 & $N$ & $<20$ \\
\hline 422337 & 100 & 1,500 & 11.0 & $N$ & 15 & 200 & 20 & 50 & $N$ & $<20$ \\
\hline 422338 & 100 & 2,000 & 1.0 & $N$ & 15 & 200 & 20 & 70 & $N$ & 20 \\
\hline 422339 & $<10$ & 200 & 41.0 & $<10$ & $N$ & $N$ & $\langle 5$ & $N$ & $N$ & $N$ \\
\hline 422340 & 100 & 2,000 & 1.0 & $N$ & 15 & 200 & 50 & 30 & $\langle 5$ & $N$ \\
\hline 422342 & 70 & 1,500 & 1.0 & $N$ & 15 & 150 & 50 & 50 & $N$ & $N$ \\
\hline 422344 & 100 & 1,500 & $\langle 1.0$ & $N$ & 20 & 200 & 30 & 50 & $N$ & 120 \\
\hline 422345 & 20 & 1,000 & 1.5 & $N$ & $N$ & 30 & 100 & $N$ & $N$ & $N$ \\
\hline 422346 & 100 & 2,000 & 11.0 & $N$ & 20 & 200 & 30 & 50 & $N$ & $N$ \\
\hline 422347 & 150 & 2,000 & 1.0 & $N$ & 30 & 300 & 30 & 20 & $<5$ & $N$ \\
\hline 422348 & 100 & 2,000 & 1.0 & $N$ & 20 & 200 & 50 & 50 & $N$ & $N$ \\
\hline 422352 & 70 & 2,000 & 1.0 & $N$ & 15 & 150 & 30 & 50 & $<5$ & $N$ \\
\hline 422353 & 70 & 2,000 & 1.0 & $N$ & 15 & 200 & 50 & 30 & $N$ & $N$ \\
\hline 422354 & 100 & 2,000 & 1.0 & $N$ & 15 & 200 & 30 & 70 & $N$ & $<20$ \\
\hline 422355 & 70 & 1,500 & 1.0 & $N$ & 20 & 150 & 50 & 30 & $N$ & $<20$ \\
\hline 422356 & 70 & 2,000 & 41.0 & $N$ & 10 & 200 & 20 & 50 & $N$ & $<20$ \\
\hline 422357 & 50 & 2,000 & $\langle 1.0$ & $N$ & 20 & 300 & 50 & 20 & $N$ & $N$ \\
\hline 422358 & $<10$ & 500 & 1.0 & $N$ & 7 & 20 & 15 & $N$ & $N$ & $N$ \\
\hline 422359 & 70 & 2,000 & 1.0 & $N$ & 15 & 150 & 30 & 50 & $N$ & $N$ \\
\hline 422360 & 100 & 2,000 & 1.0 & $N$ & 20 & 150 & 50 & 50 & $N$ & $N$ \\
\hline 422363 & 70 & 1,500 & 1.0 & $N$ & 10 & 200 & 30 & 50 & $N$ & $<20$ \\
\hline 422364 & 100 & 1,500 & 41.0 & $N$ & 15 & 150 & 20 & 50 & $N$ & 20 \\
\hline 422365 & 100 & 2,000 & 1.0 & $N$ & 20 & 300 & 20 & 50 & 7 & 20 \\
\hline $4223 b 6$ & 100 & 2,000 & 1.0 & $N$ & 20 & 300 & 30 & 50 & $N$ & 30 \\
\hline 422367 & 100 & 2,000 & 1.0 & $N$ & 15 & 150 & 20 & 50 & $N$ & $<20$ \\
\hline 422368 & 100 & 1,500 & 1.0 & $\ddot{N}$ & 15 & 150 & 20 & 50 & 5 & $<20$ \\
\hline 422369 & 100 & 2,000 & $\langle 1.0$ & $N$ & 15 & 200 & 15 & 50 & $N$ & $<20$ \\
\hline 422370 & 50 & 1,500 & 1.0 & $N$ & 15 & 100 & 50 & 30 & 7 & $N$ \\
\hline 422371 & 70 & 2,000 & $\langle 1.0$ & $N$ & 15 & 200 & 50 & 70 & 7 & $<20$ \\
\hline 422372 & 50 & 1,500 & 1.0 & $N$ & 15 & 100 & 20 & $N$ & 10 & $N$ \\
\hline 422373 & 100 & 3,000 & 1.0 & $N$ & 15 & 150 & 20 & 70 & 7 & N \\
\hline 422374 & 70 & 1,500 & 1.0 & $N$ & 15 & 100 & 30 & 50 & $\langle 5$ & $N$ \\
\hline 422375 & 70 & 1,500 & 1.0 & $N$ & 15 & 100 & 20 & 50 & $<5$ & $\mathbf{N}$ \\
\hline 422376 & 20 & 300 & 1.0 & $\mathrm{~N}$ & $N$ & 10 & 15 & $N$ & $N$ & $N$ \\
\hline 422377 & 50 & 700 & 1.0 & $N$ & 7 & 70 & 20 & $N$ & 10 & $N$ \\
\hline 422378 & 70 & 1,000 & $\langle 1.0$ & $N$ & 10 & 100 & 20 & 50 & $<5$ & $N$ \\
\hline 422379 & 70 & 1,000 & 11.0 & $N$ & 10 & 100 & 20 & 50 & $N$ & $N$ \\
\hline 422380 & 50 & 1,000 & N & $N$ & 10 & 100 & 50 & $N$ & $<5$ & $N$ \\
\hline 422381 & 50 & 1,000 & 1.0 & $N$ & 10 & 100 & 20 & 50 & $N$ & $N$ \\
\hline 422382 & 30 & 700 & 1.0 & $N$ & 7 & 50 & 20 & 20 & $N$ & $N$ \\
\hline 422383 & 50 & 1,500 & 41.0 & $N$ & 10 & 100 & 50 & 20 & N & $N$ \\
\hline 422384 & 70 & 1,000 & $\langle 1.0$ & $N$ & 15 & 100 & 50 & 20 & 5 & N \\
\hline 422385 & 30 & 700 & 1.0 & $N$ & 10 & 70 & 20 & $<20$ & 7 & $N$ \\
\hline 422387 & 50 & 1,000 & 11.0 & $N$ & 15 & 100 & 20 & 50 & $N$ & $N$ \\
\hline
\end{tabular}


TABLE 2.--RESILIS OF SPECTROGRAPHIC ANALYSES OF NURE STREAM-SEDIMENT SAMPLES FROM THE LIUENGOOD

AND HESTERN $1 / 3$ OF THE CIRCLE QUADKANGLES, ALASKA--CONTI NUEd

\begin{tabular}{|c|c|c|c|c|c|c|c|c|c|c|c|}
\hline Sample & $\begin{array}{c}\mathrm{Ni}-\mathrm{pp} \text { } \\
5\end{array}$ & $\begin{array}{c}\mathrm{Pb}-\mathrm{pp} n \\
5\end{array}$ & $\begin{array}{c}\text { St-pp } \\
5\end{array}$ & $\begin{array}{c}S n-p p l \\
5\end{array}$ & $\begin{array}{c}\text { Sr-ppl } \\
5\end{array}$ & $\begin{array}{c}V_{\text {-ppm }} \\
5\end{array}$ & $\begin{array}{c}W-p p \text { w } \\
5\end{array}$ & $\begin{array}{c}\text { Y-ppl } \\
5\end{array}$ & $\begin{array}{c}\text { In-ppo } \\
5\end{array}$ & $\begin{array}{c}2 r-p p q \\
5\end{array}$ & $\begin{array}{c}\text { Th-ppon } \\
5\end{array}$ \\
\hline 422335 & 30 & 15 & 15 & $N$ & 300 & 150 & $N$ & 30 & N & 200 & $N$ \\
\hline 422336 & 30 & 20 & 20 & $N$ & 300 & 200 & $N$ & 70 & $N$ & 1,000 & $N$ \\
\hline 422337 & 30 & 20 & 20 & $N$ & 300 & 150 & N & 50 & N & 1,000 & $N$ \\
\hline 422338 & 30 & 20 & 20 & $N$ & 300 & 150 & $N$ & 50 & $N$ & 300 & $N$ \\
\hline 422339 & 7 & $<10$ & $N$ & $N$ & $N$ & 20 & $N$ & 10 & $N$ & 20 & $N$ \\
\hline 422340 & 50 & 20 & 20 & N & 300 & 200 & $N$ & 50 & N & 300 & $N$ \\
\hline 422342 & 50 & 15 & 20 & $N$ & 200 & 150 & $N$ & 30 & $N$ & 300 & $N$ \\
\hline 422344 & 30 & 30 & 20 & $N$ & 200 & 200 & $N$ & 50 & $N$ & 700 & $N$ \\
\hline 422345 & 20 & 20 & 15 & $N$ & $\$ 100$ & 150 & $N$ & 30 & N & 100 & $N$ \\
\hline 422346 & 30 & 20 & 20 & $N$ & 300 & 300 & $N$ & 70 & $N$ & 1,000 & $N$ \\
\hline 422347 & 100 & 20 & 20 & 30 & 150 & 300 & N & 30 & $N$ & 200 & $N$ \\
\hline 422349 & 30 & 30 & 20 & $N$ & 200 & 300 & $N$ & 50 & $N$ & 300 & $N$ \\
\hline 422352 & 50 & 20 & 20 & $\$ 10$ & 200 & 200 & $N$ & 50 & $N$ & 300 & $N$ \\
\hline 422353 & 50 & 30 & 20 & $N$ & 200 & 200 & $N$ & 50 & $N$ & 200 & $N$ \\
\hline 422354 & 50 & 30 & 20 & $N$ & 300 & 200 & $N$ & 50 & $N$ & 200 & $N$ \\
\hline 422355 & 50 & 30 & 20 & $N$ & 200 & 150 & $N$ & 30 & $N$ & 150 & $N$ \\
\hline 422356 & 30 & 20 & 15 & N & 300 & 150 & $N$ & 30 & $N$ & 300 & $N$ \\
\hline 422357 & 50 & 20 & 20 & 10 & 200 & 150 & $N$ & 30 & $\langle 200$ & 200 & N \\
\hline 422358 & 20 & 10 & 5 & $N$ & 100 & 50 & $N$ & 20 & $N$ & 50 & $N$ \\
\hline 422359 & 30 & 20 & 20 & $\langle 10$ & 200 & 150 & $N$ & 50 & $\langle 200$ & 300 & $N$ \\
\hline 422360 & 50 & 20 & 20 & 15 & 200 & 150 & $N$ & 50 & $<200$ & 200 & $N$ \\
\hline 422363 & 20 & 20 & 20 & N & 200 & 200 & $N$ & 30 & $N$ & 500 & $N$ \\
\hline 422364 & 50 & 20 & 15 & 15 & 200 & 150 & N & 30 & $N$ & 500 & $N$ \\
\hline 422365 & 70 & 20 & 20 & 50 & 200 & 200 & N & 30 & $N$ & 300 & $N$ \\
\hline 422366 & 50 & 20 & 20 & $N$ & 200 & 200 & $N$ & 30 & $N$ & 300 & $N$ \\
\hline 422367 & 50 & 20 & 20 & $N$ & 300 & 200 & N & 50 & N & 300 & N \\
\hline 422368 & 50 & 30 & 15 & 20 & 200 & 150 & N & 30 & $N$ & 200 & $N$ \\
\hline 422369 & 50 & 20 & 20 & $N$ & 300 & 200 & $N$ & 50 & 300 & 300 & $N$ \\
\hline 422370 & 50 & 30 & 15 & $<10$ & 200 & 200 & N & 30 & $(200$ & 200 & $N$ \\
\hline 422371 & 50 & 30 & 15 & 50 & 300 & 200 & $N$ & 50 & N & 500 & $N$ \\
\hline 422372 & 50 & 15 & 15 & $<10$ & 200 & 150 & $N$ & 30 & $N$ & 200 & $N$ \\
\hline 422373 & 50 & 30 & 15 & 20 & 150 & 200 & $N$ & 30 & 200 & 200 & $N$ \\
\hline 422374 & 50 & 20 & 15 & 50 & 200 & 150 & $N$ & 50 & $N$ & 300 & $N$ \\
\hline 422375 & 30 & 20 & 15 & $N$ & 300 & 150 & N & 30 & N & 200 & $N$ \\
\hline 422376 & 10 & 10 & 5 & $N$ & $\langle 100$ & 70 & $N$ & $\langle 10$ & N & 100 & $N$ \\
\hline 422377 & 50 & 20 & 10 & 20 & 150 & 100 & $N$ & 20 & $N$ & 150 & $N$ \\
\hline 422378 & 30 & 15 & 15 & $\$ 10$ & 300 & 100 & $N$ & 50 & $N$ & 500 & $N$ \\
\hline 422379 & 30 & 20 & 15 & N & 200 & 150 & $N$ & 30 & $N$ & 200 & $N$ \\
\hline 422380 & 50 & 30 & 10 & 10 & 100 & 150 & $N$ & 20 & N & 300 & $N$ \\
\hline 422381 & 30 & 20 & 15 & $<10$ & 300 & 150 & $N$ & 30 & $N$ & 200 & $N$ \\
\hline 422382 & 20 & 10 & 10 & $N$ & 150 & 100 & $N$ & 20 & $N$ & 150 & $N$ \\
\hline 422383 & 30 & 15 & 10 & $N$ & 200 & 150 & $N$ & 20 & 200 & 200 & $\mathbb{N}$ \\
\hline 422384 & 50 & 20 & 10 & $N$ & 200 & 100 & N & 20 & $N$ & 200 & $N$ \\
\hline 422385 & 30 & 15 & 10 & 10 & 200 & 100 & N & 20 & N & 100 & $N$ \\
\hline 422387 & 30 & 20 & 15 & $\$ 10$ & 200 & 100 & $N$ & 30 & N & 200 & $N$ \\
\hline
\end{tabular}


TABLE 2.--RESULTS OF SPECTRDGRAPHIC ANALYSES OF NURE STREAM-SEDIMENT SAMPLES FROM THE LIVENGDOD

AHD WESTERN $1 / 3$ OF THE CIRCLE DUADRANGLES, ALA5KA-ContinUEd

\begin{tabular}{|c|c|c|c|c|c|c|c|c|c|}
\hline Sample & Latitude & Longitude & $\begin{array}{c}\text { Fe-pct. } \\
s\end{array}$ & $\begin{array}{c}\text { Mg-pet. } \\
s\end{array}$ & $\underset{s}{C a-p c t .}$ & $\underset{s}{\text { Ti-pct. }}$ & $\begin{array}{c}\text { Mn-ppin } \\
s\end{array}$ & $\begin{array}{c}A g-p p \text {. } \\
5\end{array}$ & $\begin{array}{c}A s-p p \text { f } \\
5\end{array}$ \\
\hline 422389 & 655956 & $14730 \quad 16$ & 10.00 & 2.00 & 2.0 & .700 & 700 & 6.5 & $N$ \\
\hline 422389 & 655914 & 1472747 & 5.00 & 1.00 & .7 & .500 & 500 & $H$ & $N$ \\
\hline 422390 & 655654 & 1472421 & 5.00 & 1.00 & 1.5 & .500 & 70 & $N$ & H \\
\hline 422391 & 655723 & 1472317 & 7.00 & 1.00 & 1.0 & .700 & 1,000 & $N$ & $N$ \\
\hline 422392 & 655944 & 1472025 & 5.00 & 1.50 & 2.0 & .500 & 700 & $N$ & $N$ \\
\hline 422393 & 655913 & 1471751 & 5.00 & 1.50 & 1.5 & .500 & 500 & $H$ & $N$ \\
\hline 422395 & 655940 & 1471533 & 5.00 & 1.50 & 2.0 & .500 & 500 & H & N \\
\hline 422396 & 655940 & $147 \quad 14 \quad 22$ & 5.00 & 1.50 & 1.0 & .300 & 500 & N & H \\
\hline 422397 & 655836 & 147108 & 5.00 & 1.50 & 1.5 & .500 & 1,000 & $N$ & $\mathrm{~N}$ \\
\hline 422398 & 655858 & 14737 & 3.00 & 1.50 & 2.0 & .700 & 500 & $H$ & $N$ \\
\hline 422399 & 653456 & 1484143 & 5.00 & 1.50 & 3.0 & .500 & 500 & $\mathrm{~N}$ & $H$ \\
\hline 422400 & 653535 & $14844 \quad 2$ & 3.00 & 1.50 & 3.0 & .500 & 700 & H & $H$ \\
\hline 422401 & 653524 & 148490 & 5.00 & 1.50 & 3.0 & .500 & 700 & $N$ & $H$ \\
\hline 422402 & $6535 \quad 8$ & 148513 & 5.00 & 1.50 & 1.5 & .500 & 500 & $N$ & $N$ \\
\hline 422403 & 653737 & $14856 \quad 9$ & 7.00 & 1.50 & .5 & .500 & 500 & 6.5 & $H$ \\
\hline 422404 & 653929 & 1485814 & 2.00 & 1.50 & 1.5 & .700 & 500 & $H$ & H \\
\hline 422405 & 654045 & 1485846 & 10.00 & 1.50 & 1.0 & .500 & 500 & $N$ & $N$ \\
\hline 422407 & 654118 & 1485822 & 2.00 & 1.50 & 1.5 & .500 & 500 & N & $H$ \\
\hline 422408 & 654130 & 1485636 & 5.00 & 1.50 & 2.0 & .300 & 700 & $H$ & $H$ \\
\hline 422409 & 654123 & 1485411 & 5.00 & 1.50 & 1.0 & .500 & 300 & N & $N$ \\
\hline 422410 & 654135 & $14848 \quad 42$ & 5.00 & 1.50 & 1.5 & .500 & 500 & $N$ & N \\
\hline 422411 & 654141 & 1484650 & 5.00 & 1.50 & 1.0 & .500 & 500 & N & $N$ \\
\hline 422412 & 654238 & $14843 \quad 31$ & 5.00 & 1.50 & 1.0 & .300 & 300 & $N$ & $H$ \\
\hline 422413 & 654256 & 1484136 & 5.00 & 1.50 & 3.0 & .500 & 700 & N & $N$ \\
\hline 422414 & 654256 & 1484030 & 5.00 & 2.00 & 2.0 & .700 & 500 & $N$ & H \\
\hline 422415 & 654456 & $14837 \quad 5$ & 3.00 & 1.50 & 1.5 & .700 & 300 & N & $N$ \\
\hline 422416 & $6545 \quad 2$ & $148 \quad 37 \quad 47$ & 5.00 & 1.50 & 2.0 & .500 & 500 & $N$ & $n$ \\
\hline 422417 & 654014 & 149356 & 5.00 & 2.00 & 5.0 & .500 & 700 & $N$ & $H$ \\
\hline 422418 & $6540 \quad 4$ & 149518 & 3.00 & 1.50 & 1.5 & .500 & 500 & $N$ & $N$ \\
\hline 422419 & 654033 & 1491010 & 2.00 & 1.50 & 2.0 & .300 & 700 & H & $N$ \\
\hline 422420 & 654235 & 149329 & 2.00 & 1.00 & 2.0 & .300 & 500 & N & $N$ \\
\hline 422421 & 654327 & $149 \quad 132$ & 3.00 & 1.50 & 2.0 & .700 & 500 & $N$ & $N$ \\
\hline 422422 & $65 \quad 4337$ & 1495752 & 3.00 & 2.00 & 3.0 & .700 & 1,000 & $N$ & N \\
\hline 422423 & 654354 & 1485515 & 5.00 & 1.50 & 3.0 & .700 & 1,000 & $\mathrm{~N}$ & $H$ \\
\hline 422424 & 654319 & 148541 & 5.00 & 1.50 & 2.0 & .700 & 700 & $N$ & H \\
\hline 422425 & 65461 & $148 \quad 44 \quad 44$ & 5.00 & 1.50 & 3.0 & .700 & 500 & $<.5$ & $N$ \\
\hline 422426 & 654641 & 1484246 & 3.00 & 1.50 & 3.0 & .700 & 500 & $N$ & $\mathrm{H}$ \\
\hline 422428 & 654744 & 1484147 & 3.00 & 1.50 & 2.0 & .700 & 500 & $N$ & $H$ \\
\hline 422429 & 654824 & 1484153 & 3.00 & 2.00 & 5.0 & .500 & 700 & $N$ & N \\
\hline 422430 & 654857 & 1492146 & 5.00 & 2.00 & 5.0 & .700 & 1,000 & $N$ & N \\
\hline 422431 & 655250 & $149 \div 51$ & 2.00 & 1.00 & 2.0 & .700 & 500 & $N$ & H \\
\hline 422432 & 655612 & 14976 & 5.00 & 1.50 & 3.0 & .700 & 700 & $N$ & $H$ \\
\hline 422433 & 655546 & 149156 & 7.00 & 2.00 & 5.0 & .500 & 1,000 & $N$ & $\mathrm{H}$ \\
\hline 422434 & 655629 & 1495824 & 3.00 & 1.50 & 3.0 & .700 & 500 & $H$ & $H$ \\
\hline 422435 & 655618 & 1495649 & 2.00 & 1.50 & 3.0 & .700 & 500 & N & H \\
\hline
\end{tabular}


TABLE 2.--RESULTS OF SPECTROGRAPHIC ANALYSES OF NURE STREAM-SEDIMENT SAMPLES FROM THE LIVEMGDOD

AND WESTERN $1 / 3$ OF THE CIRCLE QUADRANGLES, ALASKA--Continued

\begin{tabular}{|c|c|c|c|c|c|c|c|c|c|c|}
\hline Sample & $\begin{array}{c}\text { B-ppdi } \\
5\end{array}$ & $\begin{array}{c}\text { Ba-ppi } \\
5\end{array}$ & $\begin{array}{c}\text { Be-ppa } \\
5\end{array}$ & $\begin{array}{c}\mathrm{Bi}-\mathrm{ppa} \\
5\end{array}$ & $\begin{array}{c}\text { Co-ppa } \\
5\end{array}$ & $\begin{array}{c}C r-p p i a \\
5\end{array}$ & $\begin{array}{c}\text { Cu-pp酉 } \\
5\end{array}$ & $\begin{array}{c}\text { La-ppa } \\
5\end{array}$ & $\begin{array}{c}\text { Mo-ppi } \\
5\end{array}$ & $\begin{array}{c}\text { Wb-ppi } \\
5\end{array}$ \\
\hline 422388 & 100 & 1,000 & $\langle 1.0$ & $N$ & 20 & 200 & 20 & 70 & $N$ & $<20$ \\
\hline 422389 & 100 & 1,000 & 1.0 & $N$ & 15 & 100 & 10 & 70 & $\mathrm{~N}$ & $N$ \\
\hline 422390 & 50 & 1,000 & 11.0 & $N$ & 15 & 100 & 15 & 50 & $<5$ & $N$ \\
\hline 422391 & 50 & 1,000 & 1.0 & $n$ & 20 & 150 & 30 & 50 & $N$ & $N$ \\
\hline 422392 & 50 & 1,500 & 41.0 & $H$ & 10 & 70 & 20 & 50 & $H$ & $N$ \\
\hline 422393 & 50 & 1,000 & $\langle 1.0$ & $n$ & 15 & 100 & 20 & 50 & $\mathrm{~N}$ & $N$ \\
\hline 422395 & 70 & 1,500 & 1.0 & $N$ & 15 & 100 & 20 & 70 & $N$ & $N$ \\
\hline 422396 & 50 & 1,000 & $\langle 1.0$ & $N$ & 10 & 50 & 20 & 30 & $H$ & $N$ \\
\hline 422397 & 50 & 1,500 & $\langle 1.0$ & $N$ & 15 & 70 & 15 & 50 & $N$ & $N$ \\
\hline 422398 & 70 & 1,000 & 1.0 & $N$ & 15 & 150 & 15 & 50 & $<5$ & $<20$ \\
\hline 422399 & 70 & 1,000 & 1.0 & $H$ & 15 & 150 & 20 & 30 & $\langle 5$ & $\langle 20$ \\
\hline 422400 & 50 & 1,000 & 1.0 & $\mathrm{H}$ & 15 & 100 & 20 & 50 & $N$ & $N$ \\
\hline 422401 & 70 & 1,000 & 1.0 & $N$ & 15 & 150 & 20 & 50 & N & N \\
\hline 422402 & 70 & 1,000 & 1.0 & $N$ & 20 & 150 & 20 & 50 & $<5$ & $<20$ \\
\hline 422403 & 70 & 1,000 & 1.0 & $N$ & 20 & 200 & 70 & $N$ & $\langle 5$ & $N$ \\
\hline 422404 & 70 & 1,500 & 1.0 & $H$ & 15 & 100 & 30 & 50 & $N$ & $<20$ \\
\hline 422405 & 50 & 1,500 & 1.0 & $N$ & 10 & 100 & 30 & 50 & N & $N$ \\
\hline 422407 & 70 & 1,500 & $\langle 1.0$ & $H$ & 15 & 150 & 30 & 50 & $<5$ & $<20$ \\
\hline 422408 & 70 & 1,500 & 1.0 & $N$ & 10 & 70 & 20 & 20 & H & $N$ \\
\hline 422409 & 70 & 1,000 & 1.0 & $N$ & 15 & 150 & 20 & 30 & $N$ & $N$ \\
\hline 422410 & 100 & 1,500 & 1.0 & $N$ & 15 & 100 & 30 & 50 & $N$ & 20 \\
\hline 422411 & 70 & 1,000 & 1.0 & $\ddot{N}$ & 15 & 150 & 30 & $N$ & N & $N$ \\
\hline 422412 & 70 & 1,500 & 1.0 & $n$ & 15 & 150 & 20 & 30 & $N$ & $H$ \\
\hline 422413 & 100 & 1,500 & 1.0 & $N$ & 10 & 100 & 20 & 30 & $N$ & 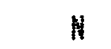 \\
\hline 422414 & 100 & 1,500 & 1.0 & $N$ & 15 & 100 & 30 & 50 & H & 20 \\
\hline 422415 & 100 & 1,500 & $\langle 1.0$ & $N$ & 7 & 150 & 20 & 30 & $N$ & $N$ \\
\hline 422416 & 70 & 1,500 & 1.0 & $N$ & 15 & 70 & 30 & 50 & $N$ & $N$ \\
\hline 422417 & 70 & 1,000 & $\langle 1.0$ & $H$ & 15 & 150 & 20 & 30 & $H$ & $N$ \\
\hline 422418 & 70 & 1,000 & 1.0 & $N$ & 10 & 100 & 20 & 50 & 45 & 20 \\
\hline 422419 & 70 & 700 & 1.0 & $N$ & 10 & 70 & 20 & $\mathrm{~N}$ & N & $N$ \\
\hline 422420 & 20 & 1,000 & 1.0 & $N$ & 7 & 50 & 20 & $N$ & $N$ & $N$ \\
\hline 422421 & 70 & 1,000 & 1.0 & $N$ & 10 & 100 & 20 & $N$ & $N$ & $N$ \\
\hline 422422 & 70 & 1,500 & 1.0 & $N$ & 15 & 100 & 15 & 30 & $H$ & $N$ \\
\hline 422423 & 70 & 1,000 & 1.0 & $N$ & 15 & 150 & 30 & 30 & $<5$ & $N$ \\
\hline 422424 & 70 & 1,500 & 1.0 & $N$ & 15 & 150 & 30 & 50 & $N$ & 20 \\
\hline 422425 & 70 & 1,000 & 1.0 & $N$ & 15 & 100 & 30 & 50 & $H$ & $N$ \\
\hline $42242 b$ & 70 & 1,000 & $\langle 1.0$ & $\mathrm{N}$ & 15 & 100 & 20 & 30 & H & $N$ \\
\hline 422428 & 70 & 1,500 & 1.0 & $N$ & 10 & 70 & 30 & 30 & $N$ & $N$ \\
\hline 422429 & 50 & 1,000 & $\langle 1.0$ & $N$ & 15 & 100 & 10 & $N$ & $N$ & $N$ \\
\hline 422430 & 70 & 1,500 & 1.0 & $N$ & 15 & 150 & 30 & 30 & H & $N$ \\
\hline 422431 & 70 & 1,000 & 1.5 & $\mathrm{H}$ & 7 & 100 & 20 & 50 & 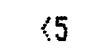 & $N$ \\
\hline 422432 & 70 & 1,500 & 1.0 & $\mu$ & 15 & 100 & 20 & 30 & $N$ & $N$ \\
\hline 422433 & 100 & 1,500 & 1.0 & $n$ & 20 & 100 & 50 & 50 & N & $\mathrm{N}$ \\
\hline 422434 & 70 & 1,000 & 1.0 & $N$ & 15 & 100 & 15 & 50 & $n$ & $<20$ \\
\hline 422435 & 70 & 1,500 & 1.0 & $H$ & 15 & 70 & 20 & 50 & $H$ & $N$ \\
\hline
\end{tabular}


TABLE 2.--RESULTS OF SPECTROGRAPHIC ANALYSES OF NUAE STREAM-SEDIMENT SAMFLES FROM THE LIVENGOOD

AND WESTERH $1 / 3$ OF THE CIRCLE QUADRANGLES, ALASKA--ContinURd

\begin{tabular}{|c|c|c|c|c|c|c|c|c|c|c|c|}
\hline Sample & $\begin{array}{c}\mathrm{Hi}-\mathrm{pp} \text { i } \\
\mathrm{s}\end{array}$ & $\begin{array}{c}\mathrm{Pb}-\mathrm{pp} \\
\vdots\end{array}$ & $\begin{array}{c}5 c-p p h \\
5\end{array}$ & $\begin{array}{c}5 n-p p \text { 角 } \\
5\end{array}$ & $\begin{array}{c}5 r-p p i n \\
s\end{array}$ & $\underset{s}{V-p p \text { m }}$ & $\begin{array}{c}W-p p h \\
5\end{array}$ & $\begin{array}{c}Y \text {-ppi } \\
s\end{array}$ & $\begin{array}{c}2 n-p p \text { I } \\
5\end{array}$ & $\begin{array}{c}2 r-p p \| \\
5\end{array}$ & $\begin{array}{c}\text { Th-ppt } \\
5\end{array}$ \\
\hline 422388 & 70 & 50 & 15 & $N$ & 300 & 200 & $H$ & 50 & 300 & 500 & $H$ \\
\hline 422389 & 30 & $<10$ & 10 & $N$ & 150 & 70 & $N$ & 30 & $H$ & 300 & $N$ \\
\hline 422390 & 20 & 15 & 15 & $<10$ & 200 & 150 & $N$ & 50 & $N$ & 500 & $N$ \\
\hline 422391 & 50 & 20 & 20 & 20 & 200 & 150 & $N$ & 50 & H & 200 & $N$ \\
\hline 422392 & 20 & 20 & 20 & 10 & 200 & 100 & H & 30 & N & 200 & 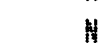 \\
\hline 422393 & 30 & 20 & 15 & $\langle 10$ & 200 & 100 & $N$ & 30 & $H$ & 150 & $H$ \\
\hline 422395 & 30 & 15 & 15 & 15 & 200 & 100 & $N$ & 30 & $H$ & 200 & $H$ \\
\hline 422396 & 20 & 10 & 10 & $N$ & 100 & 150 & $N$ & 20 & $H$ & 200 & $H$ \\
\hline 422397 & 20 & 15 & 15 & $H$ & 200 & 100 & $H$ & 30 & $N$ & 150 & $N$ \\
\hline 422398 & 30 & 15 & 15 & H & 200 & 100 & $H$ & 50 & $N$ & 500 & $H$ \\
\hline 422399 & 50 & 20 & 15 & 10 & 200 & 100 & $N$ & 30 & $N$ & 200 & $N$ \\
\hline 422400 & 30 & 15 & 15 & $N$ & 200 & 100 & $N$ & 30 & $n$ & 150 & $H$ \\
\hline 422401 & 30 & 15 & 15 & $N$ & 300 & 100 & H & 30 & $N$ & 200 & $\mathrm{~N}$ \\
\hline 422402 & 50 & 20 & 15 & N & 200 & 100 & $N$ & 50 & $N$ & 200 & $W$ \\
\hline 422403 & 70 & 20 & 20 & $N$ & $\langle 100$ & 150 & $H$ & 20 & 200 & 150 & $H$ \\
\hline 422404 & 30 & 20 & 20 & $N$ & 200 & 150 & $H$ & 20 & H & 200 & $N$ \\
\hline 422405 & 20 & 15 & 10 & N & 200 & 100 & $H$ & 15 & $N$ & 100 & $N$ \\
\hline 422407 & 30 & 20 & 15 & $H$ & 200 & 100 & $H$ & 30 & H & 200 & $N$ \\
\hline 422408 & 30 & 10 & 10 & 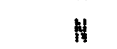 & 200 & 70 & $N$ & 30 & $H$ & 150 & $N$ \\
\hline 422409 & 50 & 20 & 10 & $\$ 10$ & 150 & 100 & $N$ & 30 & $H$ & 200 & $H$ \\
\hline 422410 & 50 & 20 & 15 & $N$ & 150 & 150 & $N$ & 50 & $N$ & 200 & $N$ \\
\hline 422411 & 50 & 20 & 15 & N & 150 & 100 & $H$ & 30 & $<200$ & 150 & $H$ \\
\hline 422412 & 50 & 20 & 15 & 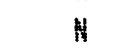 & 150 & 100 & $N$ & 30 & N & 100 & $N$ \\
\hline 422413 & 50 & 20 & 15 & $H$ & 200 & 100 & $N$ & 30 & $N$ & 150 & $N$ \\
\hline 422414 & 50 & 20 & 15 & $N$ & 200 & 150 & $N$ & 50 & $N$ & 200 & $N$ \\
\hline 422415 & 50 & 20 & 20 & 15 & 200 & 100 & $H$ & 30 & $H$ & 200 & $H$ \\
\hline 422416 & 30 & 20 & 20 & $N$ & 200 & 100 & $N$ & 30 & $N$ & 200 & $N$ \\
\hline 422417 & 30 & 15 & 15 & $N$ & 300 & 100 & $H$ & 30 & N & 150 & $N$ \\
\hline 422418 & 30 & 15 & 15 & $N$ & 200 & 100 & $N$ & 30 & $N$ & 200 & $W$ \\
\hline 422419 & 30 & 10 & 7 & N & 200 & 100 & $N$ & 15 & $N$ & 150 & $N$ \\
\hline 422420 & 20 & $<10$ & 5 & $N$ & $N$ & 100 & $N$ & 10 & $N$ & 150 & $N$ \\
\hline 422421 & 30 & 15 & 15 & $N$ & 200 & 100 & $N$ & 50 & H & 300 & $n$ \\
\hline 422422 & 30 & 20 & 15 & N & 300 & 100 & $N$ & 30 & N & 300 & $N$ \\
\hline 422423 & 50 & 15 & 15 & N & 200 & 150 & $H$ & 50 & $N$ & 300 & $N$ \\
\hline 422424 & 50 & 20 & 20 & 20 & 200 & 150 & $N$ & 50 & $H$ & 500 & $N$ \\
\hline 422425 & 50 & 15 & 15 & N & 200 & 100 & $H$ & 30 & $N$ & 200 & $N$ \\
\hline $42242 b$ & 30 & 15 & 15 & $N$ & 200 & 100 & $N$ & 30 & $H$ & 200 & $N$ \\
\hline 422428 & 30 & 20 & 15 & 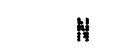 & 300 & 150 & $H$ & 30 & $N$ & 200 & $N$ \\
\hline 422429 & 30 & 15 & 15 & $N$ & 300 & 100 & $N$ & 30 & $N$ & 200 & $\ddot{N}$ \\
\hline 422430 & 50 & 20 & 15 & $N$ & 200 & 150 & $N$ & 30 & W & 300 & $N$ \\
\hline 422431 & 30 & 20 & 15 & $N$ & 200 & 100 & $N$ & 30 & $n$ & 200 & $H$ \\
\hline 422432 & 30 & 20 & 15 & $N$ & 300 & 150 & $N$ & 30 & N & 200 & $N$ \\
\hline 422433 & 50 & 30 & 20 & N & 200 & 150 & $N$ & 50 & $H$ & 150 & $N$ \\
\hline 422434 & 30 & 15 & 15 & $\mathrm{H}$ & 200 & 100 & $N$ & 30 & N & 500 & $N$ \\
\hline 422435 & 50 & 15 & 20 & $H$ & 300 & 100 & $\mathrm{H}$ & 30 & W & 150 & $N$ \\
\hline
\end{tabular}


TABLE 2.--RESULTS OF SPECTROGRAPHIC ANALYSES OF NURE STREAM-SEDIMENT SAMPLES FROM THE LIVENGOOD AND WESTERN $1 / 3$ OF THE CIRCLE QUADRANGLES, ALASKA--Continued

\begin{tabular}{|c|c|c|c|c|c|c|c|c|c|}
\hline Sample & Latitude & Longitude & $\begin{array}{c}\text { Fe-pct. } \\
s\end{array}$ & $\begin{array}{c}M_{g}-p c t . \\
s\end{array}$ & $\begin{array}{c}\text { Ca-pct. } \\
s\end{array}$ & $\begin{array}{c}\text { Ti-pct. } \\
s\end{array}$ & $\begin{array}{c}\text { Mn-ppof } \\
5\end{array}$ & $\begin{array}{c}A g-p p \text { mi } \\
s\end{array}$ & $\begin{array}{c}A 5-p p \text { in } \\
s\end{array}$ \\
\hline 422436 & 655520 & 1485456 & 3.00 & 2.00 & 5.0 & .700 & 700 & $N$ & $N$ \\
\hline 422437 & 65556 & 1485325 & 5.00 & 2.00 & 2.0 & .700 & 500 & $N$ & N \\
\hline 422438 & 655611 & $148 \quad 46 \quad 27$ & 2.00 & 1.50 & 1.5 & .500 & 300 & $N$ & $N$ \\
\hline 422439 & 655957 & $148 \quad 43 \quad 24$ & .20 & .10 & 2.0 & .015 & 70 & $<.5$ & $N$ \\
\hline 422440 & 655828 & 1485312 & 3.00 & 1.50 & 1.5 & .500 & 300 & $N$ & $N$ \\
\hline 422441 & 655940 & 1485254 & 7.00 & 2.00 & 3.0 & .700 & 1,000 & $<.5$ & $N$ \\
\hline 422442 & 655930 & 1485527 & 10.00 & 3.00 & 2.0 & .700 & 1,000 & 6.5 & $N$ \\
\hline 422443 & 655719 & $14857 \quad 12$ & 3.00 & 1.50 & 2.0 & .700 & 500 & $N$ & $N$ \\
\hline 422444 & 655751 & $149 \quad 4 \quad 17$ & 7.00 & 1.50 & 1.5 & .500 & 700 & $<.5$ & N \\
\hline 422445 & 655949 & $149 \quad 727$ & 7.00 & 3.00 & 3.0 & .700 & 1,000 & N & N \\
\hline 422446 & 655845 & $14910 \quad 6$ & 7.00 & 2.00 & 2.0 & .500 & 700 & $N$ & $N$ \\
\hline 422447 & 655942 & 1491057 & 7.00 & 1.50 & 1.0 & .700 & 500 & $<.5$ & $N$ \\
\hline 422448 & 655626 & 1491059 & 5.00 & 2.00 & 3.0 & .500 & 1,000 & N & N \\
\hline 422449 & 655638 & 1491722 & 7.00 & 2.00 & 3.0 & .700 & 1,000 & $<.5$ & $N$ \\
\hline 422450 & 655742 & 1491847 & 7.00 & 3.00 & 3.0 & .700 & 1,000 & $<.5$ & $N$ \\
\hline 422451 & 655251 & 1494955 & 3.00 & 1.50 & 1.5 & .500 & 500 & $N$ & $N$ \\
\hline 422452 & 655419 & 1494756 & 1.50 & 1.00 & 2.0 & .300 & 200 & $<.5$ & $N$ \\
\hline 422453 & 655559 & 149549 & 5.00 & 1.50 & 2.0 & .500 & 500 & $N$ & $N$ \\
\hline 422454 & 655433 & $14958 \quad 45$ & 3.00 & 1.50 & 1.5 & .500 & 500 & N & $N$ \\
\hline 422455 & 655331 & $14959 \quad 17$ & 2.00 & 1.50 & 2.0 & .500 & 300 & $N$ & $N$ \\
\hline 422457 & 655330 & 1495133 & 5.00 & 1.50 & 1.5 & .500 & 300 & N & $N$ \\
\hline 422458 & $6550 \quad 17$ & 1494336 & 5.00 & 2.00 & 3.0 & .500 & 1,000 & N & $N$ \\
\hline 422459 & 654144 & 1493924 & 5.00 & 2.00 & 3.0 & .500 & 1,000 & $N$ & $N$ \\
\hline 422460 & 653915 & $14943 \quad 15$ & 3.00 & 1.50 & 3.0 & .500 & 500 & $N$ & $N$ \\
\hline 422462 & $6543 \quad 4$ & 149323 & 3.00 & 1.50 & 1.0 & .500 & 500 & $<.5$ & N \\
\hline 422463 & 654345 & 1492827 & 5.00 & 1.50 & 1.0 & .500 & 500 & $<.5$ & $N$ \\
\hline 422464 & 654255 & $14922 \quad 17$ & 5.00 & 1.50 & 2.0 & .500 & 700 & $<.5$ & $N$ \\
\hline 422465 & 654046 & 149221 & 5.00 & 1.50 & 2.0 & .500 & 500 & $N$ & $N$ \\
\hline 422466 & 65418 & 1492733 & 5.00 & 1.50 & 1.5 & .500 & 1,000 & N & N \\
\hline 422467 & 65397 & 1493126 & 5.00 & 1.50 & 1.5 & .500 & 500 & $N$ & $N$ \\
\hline 422469 & 653830 & $14937 \quad 12$ & 5.00 & 1.50 & 1.5 & .500 & 500 & N & $N$ \\
\hline 422470 & 653736 & 1493830 & 3.00 & 1.50 & 2.0 & .500 & 300 & $N$ & $N$ \\
\hline 422471 & 653520 & 1493245 & 3.00 & 1.50 & 3.0 & .500 & 500 & N & N \\
\hline 422472 & 653547 & 1492958 & 3.00 & 1.50 & 2.0 & .500 & 300 & $N$ & $N$ \\
\hline 422473 & 653456 & 1492036 & 2.00 & 1.50 & 2.0 & .500 & 300 & N & $N$ \\
\hline 422475 & 653445 & $149 \quad 858$ & 3.00 & 1.50 & 2.0 & .500 & 300 & $N$ & $N$ \\
\hline 422476 & 65353 & $149 \quad 527$ & 3.00 & 1.50 & 3.0 & .500 & 500 & $N$ & $N$ \\
\hline 422478 & $6531 \quad 15$ & 1485559 & 7.00 & 1.50 & 1.0 & .500 & 1,000 & $<.5$ & $N$ \\
\hline 422479 & 655259 & 1491320 & 3.00 & 1.50 & 1.5 & .500 & 500 & $N$ & $N$ \\
\hline 422480 & 655335 & 1491318 & 3.00 & 1.50 & 5.0 & .300 & 700 & $N$ & $N$ \\
\hline 422481 & 65513 & 1495049 & 5.00 & 1.00 & 2.0 & .300 & 700 & $N$ & $N$ \\
\hline 422482 & 653651 & 1495232 & 2.00 & 1.00 & 1.0 & .300 & 500 & N & $N$ \\
\hline 422483 & 654324 & 1494852 & 2.00 & 1.00 & 1.0 & .200 & 500 & N & $N$ \\
\hline 422484 & 654354 & 1494539 & .10 & .05 & .5 & .020 & 200 & $N$ & $N$ \\
\hline 422485 & $6545 \quad 8$ & 1494622 & 1.50 & .70 & .7 & .200 & 700 & $N$ & N \\
\hline
\end{tabular}


TABLE 2.--RESULTS OF SPECTROGRAPHIC ANALYSES OF NURE STREAM-SEDIMENT SAMPLES FROM THE LIVENGOOD AND WESTERN $1 / 3$ OF THE CIRCLE QUADRANGLES, ALASKA--ContinUed

\begin{tabular}{|c|c|c|c|c|c|c|c|c|c|c|}
\hline Sa解le & $\begin{array}{c}\text { E-pplif } \\
\Xi\end{array}$ & $\begin{array}{c}\text { Ba-ppi } \\
5\end{array}$ & $\begin{array}{c}\text { Be-ppin } \\
5\end{array}$ & $\begin{array}{c}\mathrm{Bi}-\mathrm{pp} \text { 用 } \\
5\end{array}$ & $\begin{array}{c}\text { Co-ppi } \\
5\end{array}$ & $\begin{array}{c}\mathrm{Cr}-\mathrm{pp} \text { m } \\
5\end{array}$ & $\begin{array}{c}\text { Cu-ppl } \\
5\end{array}$ & $\begin{array}{l}\text { La-ppin } \\
\quad 5\end{array}$ & $\begin{array}{l}\text { Mo-ppla } \\
5\end{array}$ & $\begin{array}{c}\mathrm{Nb}-\mathrm{pp} \text { 酉 } \\
5\end{array}$ \\
\hline 422436 & 70 & 1,500 & $\langle 1.0$ & $\mathbb{N}$ & 15 & 100 & 30 & 50 & $N$ & $N$ \\
\hline 422437 & 100 & 1,500 & $\langle 1.0$ & $\mathbf{N}$ & 20 & 100 & 50 & 50 & 7 & $N$ \\
\hline 422438 & 70 & 1,000 & 1.0 & $N$ & 10 & 100 & 30 & $H$ & $\langle 5$ & $N$ \\
\hline 422439 & $<10$ & 100 & 11.0 & $N$ & $N$ & $<10$ & 5 & 20 & $N$ & $N$ \\
\hline 422440 & 50 & 1,000 & 1.0 & $N$ & 15 & 70 & 50 & 30 & $\langle 5$ & $\mathrm{N}$ \\
\hline 422441 & 100 & 1,500 & 1.0 & $N$ & 20 & 150 & 50 & 50 & $N$ & $<20$ \\
\hline 422442 & 100 & 1,500 & $\langle 1.0$ & $N$ & 20 & 200 & 100 & $N$ & $<5$ & $<20$ \\
\hline 422443 & 70 & 1,500 & $\langle 1.0$ & $N$ & 10 & 150 & 20 & 50 & $N$ & $<20$ \\
\hline 422444 & 100 & 1,500 & 1.5 & $N$ & 15 & 100 & 50 & 50 & $<5$ & 20 \\
\hline 422445 & 70 & 1,000 & 41.0 & $N$ & 20 & 150 & 50 & 50 & $<5$ & $N$ \\
\hline $42244 b$ & 70 & 1,000 & 1.0 & $N$ & 20 & 150 & 50 & 30 & $N$ & $\langle 20$ \\
\hline 422447 & 150 & 1,500 & 1.5 & $N$ & 15 & 150 & 50 & 50 & $N$ & 20 \\
\hline 422448 & 70 & 1,000 & 1.0 & $N$ & 15 & 100 & 30 & 50 & $\langle 5$ & $<20$ \\
\hline 422449 & 100 & 1,500 & $\langle 1.0$ & $N$ & 20 & 150 & 50 & 50 & $<5$ & $N$ \\
\hline 422450 & 100 & 1,000 & 1.0 & $N$ & 20 & 200 & 50 & 50 & $N$ & $<20$ \\
\hline 422451 & 70 & 1,000 & 1.5 & $N$ & 10 & 70 & 20 & $N$ & $N$ & $N$ \\
\hline 422452 & 50 & 700 & 1.0 & $N$ & 7 & 50 & 30 & $N$ & $\longleftrightarrow 5$ & $N$ \\
\hline 422453 & 70 & 1,000 & 1.0 & $N$ & 15 & 150 & 30 & 30 & $N$ & $N$ \\
\hline 422454 & 100 & 1,000 & 1.5 & $N$ & 15 & 70 & 30 & 50 & N & $<20$ \\
\hline 422455 & 70 & 1,000 & 1.0 & $N$ & 10 & 70 & 30 & 50 & $\langle 5$ & $N$ \\
\hline 422457 & 100 & 1,000 & 1.0 & $N$ & 10 & 150 & 50 & 50 & $\langle 5$ & $N$ \\
\hline 422458 & 50 & 1,000 & 11.0 & $N$ & 15 & 150 & 30 & 30 & $N$ & $N$ \\
\hline 422459 & 50 & 1,000 & 1.0 & $N$ & 15 & 100 & 30 & 50 & $N$ & $<20$ \\
\hline 422460 & 70 & 1,000 & 1.0 & $\mathrm{~N}$ & 15 & 150 & 15 & 30 & $\mathrm{~N}$ & $N$ \\
\hline 422462 & 70 & 1,000 & 1.0 & $<10$ & 15 & 100 & 20 & 50 & $\langle 5$ & $N$ \\
\hline 422463 & 100 & 1,000 & 1.0 & $N$ & 15 & 150 & 30 & 50 & $N$ & $N$ \\
\hline 422464 & 70 & 1,000 & 1.0 & $N$ & 15 & 100 & 20 & 30 & $\mathrm{~N}$ & $\mathrm{~N}$ \\
\hline 422465 & 70 & 1,000 & 1.0 & $N$ & 15 & 100 & 20 & 30 & $N$ & $<20$ \\
\hline $4224 b b$ & 70 & 1,000 & $\langle 1.0$ & $N$ & 15 & 200 & 15 & 20 & $N$ & $N$ \\
\hline 422467 & 70 & 1,000 & 1.0 & $N$ & 15 & 100 & 20 & 50 & $N$ & $<20$ \\
\hline 422469 & 100 & 1,000 & 1.0 & $N$ & 15 & 100 & 20 & 50 & $\mathrm{H}$ & $\langle 20$ \\
\hline 422470 & 70 & 1,000 & 1.0 & $N$ & 15 & 70 & 15 & 50 & $N$ & $<20$ \\
\hline 422471 & 70 & 1,000 & 1.5 & $N$ & 10 & 70 & 20 & 30 & $N$ & $N$ \\
\hline 422472 & 70 & 1,000 & 1.0 & $N$ & 10 & 100 & 20 & 50 & $N$ & $N$ \\
\hline 422473 & 70 & 1,000 & 1.0 & $N$ & 7 & 70 & 15 & 50 & $N$ & $<20$ \\
\hline 422475 & 70 & 1,000 & 1.0 & $N$ & 15 & 70 & 20 & 50 & $\langle 5$ & $N$ \\
\hline $42247 b$ & 70 & 1,500 & $\$ 1.0$ & $N$ & 15 & 100 & 20 & 50 & $N$ & $<20$ \\
\hline 422478 & 100 & 1,500 & 1.0 & $N$ & 20 & 150 & 30 & 50 & $\langle 5$ & $<20$ \\
\hline 422479 & 70 & 1,000 & 1.0 & $N$ & 15 & 100 & 20 & 30 & $<5$ & $N$ \\
\hline 422480 & 50 & 1,000 & 1.0 & $N$ & 15 & 70 & 20 & $N$ & $\langle 5$ & $N$ \\
\hline 422481 & 70 & 1,000 & 1.0 & $N$ & 15 & 70 & 20 & 30 & 5 & $N$ \\
\hline 422482 & 70 & 1,000 & $\langle 1.0$ & $N$ & 15 & 70 & 20 & 50 & $N$ & $N$ \\
\hline $4224 B B$ & 70 & 1,000 & 11.0 & $N$ & 10 & 70 & 20 & 50 & $N$ & $N$ \\
\hline 422484 & $<10$ & 150 & $\langle 1.0$ & $N$ & 65 & $<10$ & 7 & $N$ & $N$ & $N$ \\
\hline 422485 & 50 & 1,000 & $\$ 1.0$ & $N$ & 5 & 20 & 20 & $N$ & $N$ & $N$ \\
\hline
\end{tabular}


TABLE 2. - RESULTS OF SPECTROGRAPHIC ANALYSES OF NURE STREAM-SEDIMENT SAMPLES FROM THE LIVENGODD

AND WESTERH $1 / 3$ OF THE CIRCLE QUADRANGLES, ALASKA--Continued

\begin{tabular}{|c|c|c|c|c|c|c|c|c|c|c|c|}
\hline Sample & $\begin{array}{l}\text { Hi-pp面 } \\
\quad s\end{array}$ & $\begin{array}{c}\mathrm{Pb}-p \mathrm{p} \text { 面 } \\
\mathrm{s}\end{array}$ & $\begin{array}{c}\text { Sc-ppi } \\
s\end{array}$ & $\begin{array}{c}\text { Sn-pp fi } \\
5\end{array}$ & $\begin{array}{c}5 r-p p \text { 而 } \\
5\end{array}$ & $\underset{5}{V-p p m}$ & $\begin{array}{c}W-p p \text { in } \\
5\end{array}$ & $\begin{array}{c}Y-p p \text { m } \\
s\end{array}$ & $\begin{array}{c}2 n-p p n \\
5\end{array}$ & $\begin{array}{c}2 r-p p q \\
s\end{array}$ & $\begin{array}{c}\text { Th-pp⿴ } \\
5\end{array}$ \\
\hline 422436 & 50 & 20 & 15 & $H$ & 300 & 150 & $N$ & 50 & H & 200 & $N$ \\
\hline 422437 & 70 & 30 & 20 & $H$ & 200 & 150 & $N$ & 50 & H & 150 & $H$ \\
\hline 422438 & 50 & 15 & 20 & $N$ & 200 & 100 & $N$ & 30 & $<200$ & 150 & $H$ \\
\hline 422439 & $N$ & $\mathrm{~N}$ & $N$ & $N$ & N & 15 & $N$ & $N$ & N & 10 & $\mathrm{~N}$ \\
\hline$\$ 22440$ & 50 & 15 & 10 & 20 & 150 & 150 & $N$ & 20 & $N$ & 150 & $H$ \\
\hline 422441 & 50 & 30 & 15 & $H$ & 200 & 200 & $\mathrm{~N}$ & 30 & $N$ & 150 & $N$ \\
\hline 422442 & 50 & 50 & 20 & $\mathrm{H}$ & $<100$ & 300 & $\mathrm{H}$ & 30 & H & 150 & $N$ \\
\hline 422443 & 20 & 20 & 15 & $\mathrm{H}$ & 200 & 150 & $n$ & 30 & $n$ & 200 & 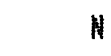 \\
\hline 422444 & 50 & 30 & 20 & $N$ & 150 & 200 & $\mathrm{H}$ & 30 & $N$ & 150 & $N$ \\
\hline 422445 & 50 & 20 & 15 & $N$ & 300 & 150 & $N$ & 30 & $N$ & 150 & $N$ \\
\hline 422446 & 50 & 30 & 15 & 10 & 200 & 200 & $\mathrm{H}$ & 30 & $H$ & 200 & $N$ \\
\hline 422447 & 50 & 30 & 20 & $\langle 10$ & 200 & 200 & $N$ & 30 & $<200$ & 200 & $H$ \\
\hline 422448 & 50 & 20 & 15 & $N$ & 300 & 200 & $N$ & 30 & $H$ & 200 & $H$ \\
\hline 422449 & 50 & 30 & 20 & $N$ & 300 & 200 & $\mathrm{H}$ & 30 & $\mathrm{H}$ & 150 & $N$ \\
\hline 422450 & 50 & 30 & 20 & $N$ & 200 & 200 & $N$ & 30 & $\mathrm{H}$ & 200 & $N$ \\
\hline 422451 & 30 & 20 & 20 & $\mathrm{H}$ & 200 & 150 & $N$ & 30 & $N$ & 150 & $N$ \\
\hline 422452 & 30 & 10 & 10 & 20 & 100 & 100 & $N$ & 20 & $H$ & 150 & $\mathrm{H}$ \\
\hline 422453 & 30 & 15 & 15 & $\mathrm{H}$ & 200 & 200 & $N$ & 30 & $H$ & 300 & $\mathrm{H}$ \\
\hline 422454 & 30 & 30 & 15 & $N$ & 200 & 150 & $\mathrm{H}$ & 30 & $N$ & 300 & $N$ \\
\hline 422455 & 30 & 15 & 15 & $\mathrm{H}$ & 150 & 150 & $N$ & 20 & $\mathrm{H}$ & 150 & $N$ \\
\hline 422457 & 50 & 50 & 15 & 20 & 150 & 150 & $H$ & 20 & $N$ & 200 & $N$ \\
\hline 422458 & 30 & 15 & 10 & $\mathrm{H}$ & 500 & 150 & $\mathrm{H}$ & 20 & $N$ & 300 & $\mathrm{H}$ \\
\hline 422459 & 30 & 15 & 10 & $N$ & 300 & 150 & $N$ & 20 & $H$ & 300 & 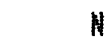 \\
\hline 422460 & 30 & 15 & 15 & $N$ & 200 & 100 & $\mathrm{H}$ & 30 & $\mathrm{H}$ & 150 & $N$ \\
\hline 422462 & 50 & 20 & 15 & 15 & 200 & 100 & $n$ & 30 & $<200$ & 150 & $N$ \\
\hline 422463 & 50 & 20 & $\mathrm{H}$ & $H$ & 150 & 100 & $H$ & 20 & $\mathrm{H}$ & 150 & $N$ \\
\hline 422464 & 50 & 20 & 15 & $\mathrm{~N}$ & 200 & 100 & $\mathrm{H}$ & 20 & $\mathrm{~N}$ & 150 & 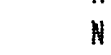 \\
\hline 422465 & 50 & 20 & 20 & $\mathrm{H}$ & 200 & 150 & $H$ & 30 & $N$ & 200 & $N$ \\
\hline 422466 & 50 & 15 & 15 & $N$ & 200 & 100 & $n$ & 20 & $N$ & 150 & $N$ \\
\hline 422467 & 50 & 20 & 15 & $N$ & 200 & 150 & $\mathrm{H}$ & 30 & H & 200 & $N$ \\
\hline 422469 & 50 & 20 & 15 & $\mathrm{~N}$ & 200 & 150 & $N$ & 30 & $N$ & 200 & $N$ \\
\hline 422470 & 50 & 15 & 15 & $\mathrm{H}$ & 200 & 100 & $N$ & 30 & N & 200 & $\mathrm{H}$ \\
\hline 422471 & 30 & 15 & 15 & $\mathrm{H}$ & 200 & 100 & $N$ & 20 & $\mathrm{H}$ & 150 & $N$ \\
\hline 422472 & 30 & 15 & 15 & $N$ & 200 & 100 & $N$ & 30 & H & 200 & $N$ \\
\hline 422473 & 30 & 15 & 15 & $N$ & 200 & 100 & $N$ & 30 & $N$ & 300 & $N$ \\
\hline 422475 & 30 & 20 & 15 & $N$ & 200 & 100 & $N$ & 30 & H & 200 & $N$ \\
\hline $42247 b$ & 30 & 15 & 15 & $N$ & 300 & 150 & $N$ & 30 & $\mathrm{H}$ & 200 & $N$ \\
\hline 422478 & 50 & 30 & 20 & $\mathrm{H}$ & 200 & 150 & $N$ & 30 & $N$ & 200 & $N$ \\
\hline 422479 & 30 & 20 & 15 & $H$ & 200 & 150 & $\mathrm{H}$ & 30 & $N$ & 200 & $N$ \\
\hline 422480 & 50 & 15 & 15 & $\Delta 10$ & 200 & 100 & $N$ & 20 & $N$ & 100 & $N$ \\
\hline 422481 & 50 & 15 & 10 & $N$ & 200 & 100 & $\mathrm{H}$ & 20 & $N$ & 150 & $N$ \\
\hline 422482 & 30 & 20 & 20 & 10 & 300 & 100 & $N$ & 30 & $H$ & 200 & $N$ \\
\hline 422483 & 30 & 20 & 15 & $N$ & 200 & 100 & $\mathrm{H}$ & 30 & $\mathrm{H}$ & 150 & $N$ \\
\hline 422484 & 5 & 10 & $<5$ & $\mathrm{H}$ & $<100$ & 15 & $n$ & $N$ & H & 20 & $N$ \\
\hline 422485 & 20 & 15 & 10 & $N$ & 150 & 100 & $N$ & 20 & $N$ & 100 & $H$ \\
\hline
\end{tabular}


TABLE 2.--RESUL TS OF SFECTROGRAPHIC AMALYSES OF NURE STREAM-SEDIMENT SAMPLES FROM THE LIVENGOOD

AND WESTERN $1 / 3$ OF THE CIRCLE QUADRANGLES, ALASKA--ContinuEd

\begin{tabular}{|c|c|c|c|c|c|c|c|c|c|}
\hline Sample & Latitude & Longitude & $\begin{array}{c}\text { Fe-pct. } \\
5\end{array}$ & $\begin{array}{c}\text { Mg-pct. } \\
5\end{array}$ & $\underset{s}{C a-p c t .}$ & $\begin{array}{c}\text { Ti-pct. } \\
s\end{array}$ & $\begin{array}{c}\text { Mn-ppm } \\
s\end{array}$ & $\begin{array}{c}\text { Ag-ppn } \\
5\end{array}$ & $\begin{array}{c}A s-p p m \\
s\end{array}$ \\
\hline $42248 b$ & 654539 & 1495559 & 1.50 & 1.00 & 1.0 & .300 & 300 & $N$ & $H$ \\
\hline 422487 & 654526 & 1495335 & 3.00 & 1.00 & 1.5 & .500 & 500 & $<.5$ & $N$ \\
\hline 422488 & 652120 & 1495829 & 2,00 & .70 & 1.0 & .500 & 300 & $N$ & $N$ \\
\hline 422489 & 652018 & 1495855 & 3.00 & 1.50 & 2.0 & .500 & 500 & $N$ & H \\
\hline 422490 & 651134 & 1495559 & .50 & .15 & 1.5 & .050 & 200 & $N$ & $N$ \\
\hline 422491 & 651220 & 149573 & 2.00 & 1.50 & 2.0 & .500 & 500 & $<.5$ & $N$ \\
\hline 422492 & 651319 & $14956 \quad 17$ & 2.00 & 1.00 & 1.0 & .500 & 500 & $N$ & $N$ \\
\hline 422493 & 651342 & 1495920 & 1.00 & .70 & .5 & .200 & 200 & N & $N$ \\
\hline 422494 & 651426 & 1495850 & 2.00 & 1.00 & .7 & .500 & 300 & $<.5$ & $N$ \\
\hline 422495 & 651525 & 1495517 & 2.00 & 1.00 & .7 & .200 & 200 & 6.5 & N \\
\hline 422496 & 651713 & 1495433 & 2.00 & 1.00 & 1.5 & .300 & 500 & N & $N$ \\
\hline 422497 & 651639 & 1495311 & 3.00 & 1.50 & 3.0 & .200 & 500 & $N$ & $N$ \\
\hline 422498 & 651749 & $14953 \quad 44$ & 3.00 & 1.50 & 2.0 & .300 & 500 & N & $N$ \\
\hline 422499 & 65185 & 149498 & 3.00 & 1.50 & 2.0 & .300 & 500 & $N$ & $N$ \\
\hline 422500 & 651947 & 1495112 & 2.00 & 1.00 & 2.0 & .300 & 500 & N & H \\
\hline 422501 & 652130 & 1494659 & 3.00 & 1.50 & 2.0 & .500 & 500 & $N$ & $N$ \\
\hline 422502 & 652059 & 1494544 & 3.00 & 1.00 & 1.0 & .500 & 500 & $<.5$ & $N$ \\
\hline 422503 & 652121 & 1494336 & 2.00 & .50 & .7 & .200 & 200 & $N$ & $N$ \\
\hline 422504 & 652249 & 1494127 & 2.00 & 1.00 & 1.0 & .500 & 200 & $N$ & N \\
\hline 422505 & 652451 & 1494257 & 3.00 & .70 & .7 & .700 & 300 & 1.0 & $N$ \\
\hline 422506 & 652456 & $14940 \quad 8$ & 2.00 & .50 & .3 & .300 & 1,000 & 2.0 & N \\
\hline 422507 & 652651 & $14940 \quad 0$ & 2.00 & .70 & .2 & .500 & 200 & $N$ & $N$ \\
\hline 422508 & 652926 & 1493955 & 3.00 & 1.00 & 1.0 & .700 & 500 & N & N \\
\hline 422509 & 652943 & 1493529 & 3.00 & 1.00 & 1.0 & .700 & 1,000 & N & H \\
\hline 422510 & 652944 & 149298 & 5.00 & 1.00 & 1.5 & .500 & 300 & 4.5 & N \\
\hline 422511 & 652331 & 1491945 & 5.00 & 1.50 & 1.5 & .700 & 300 & 4.5 & $N$ \\
\hline 422512 & 652245 & 1492215 & 7.00 & 1.50 & 1.0 & .500 & 300 & .5 & $N$ \\
\hline 422513 & 65224 & 1492452 & 7.00 & 1.50 & 1.5 & .500 & 700 & $<.5$ & $N$ \\
\hline 422514 & 652044 & 1492943 & 5.00 & 1.50 & .5 & .500 & 700 & .5 & $N$ \\
\hline 422515 & 652011 & 1493244 & 7.00 & 2.00 & 1.0 & .500 & 500 & $\{.5$ & $N$ \\
\hline 422516 & 651838 & 1493441 & 5.00 & 1.00 & .7 & .500 & 500 & .5 & $N$ \\
\hline 422517 & 651659 & 1493853 & 7.00 & 1.50 & 1.0 & .500 & 500 & 8.5 & $N$ \\
\hline 422518 & 651147 & 1494233 & 7.00 & 1.50 & .7 & .500 & 1,500 & 6.5 & $N$ \\
\hline 422519 & $65 \quad 829$ & 1495231 & 10.00 & 1.50 & 1.0 & .500 & 500 & .5 & N \\
\hline 422520 & $65 \quad 738$ & $14955 \quad 2$ & 7.00 & 1.00 & 1.0 & .500 & 500 & $<.5$ & N \\
\hline 422521 & $65 \quad 639$ & 1494416 & 7.00 & 1.50 & 1.0 & .700 & 700 & 2.0 & N \\
\hline 422522 & 653146 & $149 \quad 256$ & 2.00 & 1.00 & 1.0 & .300 & 500 & 6.5 & $\mathrm{~N}$ \\
\hline 422523 & 65325 & 149856 & 2.00 & 1.00 & 1.0 & .500 & 500 & N & N \\
\hline 422524 & 653240 & 1492050 & 1.50 & 1.00 & .7 & .500 & 300 & 4.5 & N \\
\hline 422525 & $6532 \mathrm{lb}$ & $14927 \quad 6$ & 5.00 & 2.00 & 1.0 & .500 & 700 & $<.5$ & N \\
\hline 422526 & $65 \quad 32 \quad 17$ & $14934 \quad 14$ & 2.00 & 1.00 & 1.0 & .500 & 500 & $<.5$ & N \\
\hline 422527 & 653150 & 1495718 & 3.00 & 1.00 & 1.0 & .500 & 500 & $N$ & $N$ \\
\hline 422528 & 653433 & 1495854 & 2.00 & 1.00 & .7 & .500 & 500 & N & $N$ \\
\hline 422529 & 65373 & 1495917 & 3.00 & 1.00 & 1.0 & .500 & 300 & <.5 & $N$ \\
\hline 422530 & 653912 & 1495922 & 2.00 & 1.00 & 1.0 & .500 & 300 & 6.5 & $\mathbf{N}$ \\
\hline
\end{tabular}


TABLE 2.--RESILLTS OF SPECTRDGRAPHIC ANALYSES OF NURE STREAM-SEDIMENT SAMPLES FROM THE LIVENGOOD

AND HESTERN $1 / 3$ OF THE CIRCLE QUUARANGLES, ALASKA--Continued

\begin{tabular}{|c|c|c|c|c|c|c|c|c|c|c|}
\hline Sample & $\begin{array}{c}\text { B-ppm } \\
s\end{array}$ & $\begin{array}{c}\text { Ba-ppot } \\
\quad 5\end{array}$ & $\begin{array}{c}\text { Be-ppi } \\
5\end{array}$ & $\begin{array}{c}B i-p p q \\
\Xi\end{array}$ & $\begin{array}{c}\text { Co-ppi } \\
5\end{array}$ & $\begin{array}{c}\text { Cr-ppa } \\
s\end{array}$ & $\begin{array}{c}\text { Cu-pp } \\
5\end{array}$ & $\begin{array}{l}\text { La-ppa } \\
\quad s\end{array}$ & $\begin{array}{c}\text { Mo-ppm } \\
s\end{array}$ & $\begin{array}{c}N b-p p \text { i } \\
5\end{array}$ \\
\hline 422486 & 70 & 1,000 & $\langle 1.0$ & $N$ & 10 & 70 & 20 & 30 & $N$ & $N$ \\
\hline 422487 & 70 & 1,500 & $<1.0$ & $N$ & 15 & 100 & 20 & 50 & $N$ & $N$ \\
\hline 422488 & 50 & 700 & $\$ 1.0$ & $N$ & 7 & 50 & 15 & 20 & $N$ & $N$ \\
\hline 422489 & 100 & 1,000 & $<1.0$ & $N$ & 15 & 70 & 20 & 30 & $N$ & $N$ \\
\hline 422490 & 10 & 300 & 41.0 & $N$ & $<5$ & $\langle 10$ & 7 & $<20$ & $N$ & $N$ \\
\hline 422491 & 50 & 1,000 & $\langle 1.0$ & $N$ & 15 & 100 & 20 & 50 & $N$ & $N$ \\
\hline 422492 & 70 & 700 & $\langle 1.0$ & $N$ & 10 & 100 & 20 & $N$ & $N$ & $N$ \\
\hline 422493 & 70 & 1,000 & 1.0 & $N$ & 5 & 50 & 15 & $N$ & $N$ & $N$ \\
\hline 422494 & 100 & 1,500 & $\langle 1.0$ & $N$ & 10 & 50 & 20 & 50 & $N$ & $N$ \\
\hline 422495 & 70 & 1,000 & 1.0 & $N$ & 10 & 70 & 20 & 30 & $<5$ & $N$ \\
\hline 422496 & 70 & 1,500 & 11.0 & $N$ & 10 & 50 & 20 & 50 & $N$ & $N$ \\
\hline 422497 & 70 & 1,500 & $(1.0$ & $\mathbf{N}$ & 10 & 200 & 20 & 50 & $N$ & $N$ \\
\hline 422498 & 70 & 1,500 & 41.0 & $N$ & 10 & 70 & 15 & 30 & $N$ & $N$ \\
\hline 422499 & 70 & 1,500 & $\langle 1.0$ & $N$ & 10 & 50 & 20 & 50 & N & $N$ \\
\hline 422500 & 50 & 1,000 & $\langle 1.0$ & $N$ & 7 & 70 & 15 & 20 & $N$ & $N$ \\
\hline 422501 & 70 & 1,500 & $\$ 1.0$ & $N$ & 10 & 150 & 20 & 50 & N & $N$ \\
\hline 422502 & 70 & 1,500 & $\langle 1.0$ & $N$ & 15 & 100 & 20 & 30 & $N$ & $N$ \\
\hline 422503 & 30 & 500 & 11.0 & $N$ & 5 & 50 & 10 & $N$ & $N$ & $N$ \\
\hline 422504 & 70 & 1,000 & $\langle 1.0$ & $N$ & 10 & 100 & 20 & 30 & 5 & $N$ \\
\hline 422505 & 100 & 1,000 & $\langle 1.0$ & $N$ & 20 & 70 & 20 & 30 & 45 & $N$ \\
\hline 422506 & 100 & 1,000 & 41.0 & $N$ & 15 & 50 & 20 & 20 & $N$ & $N$ \\
\hline 422507 & 50 & 1,000 & $N$ & $N$ & 10 & 50 & 15 & 50 & N & N \\
\hline $42250 \mathrm{~B}$ & 70 & 1,500 & 11.0 & $N$ & 15 & 150 & 15 & 50 & $N$ & $<20$ \\
\hline 422509 & 70 & 1,500 & $\langle 1.0$ & $N$ & 20 & 200 & 30 & 70 & $<5$ & 20 \\
\hline 422510 & 70 & 1,500 & $\langle 1.0$ & $N$ & 20 & 200 & 30 & 50 & $\langle 5$ & $N$ \\
\hline 422511 & 100 & 1,500 & $N$ & $N$ & 20 & 200 & 50 & 30 & $<5$ & $N$ \\
\hline 422512 & 100 & 2,000 & $\langle 1.0$ & $N$ & 20 & 200 & 50 & 70 & $N$ & $N$ \\
\hline 422513 & 70 & 1,500 & $<1.0$ & $N$ & 20 & 200 & 50 & 50 & $\langle 5$ & $N$ \\
\hline 422514 & 70 & 1,500 & 11.0 & $N$ & 15 & 200 & 30 & 50 & 4 & $N$ \\
\hline 422515 & 70 & 1,500 & $\langle 1.0$ & $N$ & 20 & 200 & 50 & 50 & $N$ & $N$ \\
\hline 422516 & 70 & 2,000 & $\langle 1.0$ & $N$ & 15 & 100 & 50 & 100 & 15 & $N$ \\
\hline 422517 & 70 & 2,000 & $\$ 1.0$ & $N$ & 20 & 300 & 30 & 100 & 5 & $N$ \\
\hline 422518 & 200 & 2,000 & 1.0 & $N$ & 20 & 100 & 50 & 30 & 10 & 20 \\
\hline 422519 & 150 & 3,000 & $\langle 1.0$ & $N$ & 20 & 300 & 50 & 100 & 5 & $<20$ \\
\hline 422520 & 100 & 2,000 & $\langle 1.0$ & $N$ & 20 & 150 & 20 & 50 & $<5$ & $<20$ \\
\hline 422521 & 150 & 3,000 & $(1.0$ & $N$ & 20 & 300 & 100 & 50 & 15 & $<20$ \\
\hline 422522 & 70 & 1,000 & $\langle 1.0$ & $\ddot{N}$ & 15 & 100 & 20 & 50 & $N$ & $N$ \\
\hline 422523 & 100 & 1,000 & $\$ 1.0$ & $N$ & 20 & 150 & 20 & 70 & $N$ & $N$ \\
\hline 422524 & 100 & 1,000 & $\langle 1.0$ & $N$ & 10 & 50 & 15 & 30 & $N$ & $N$ \\
\hline 422525 & 100 & 1,500 & 11.0 & $N$ & 20 & 100 & 30 & 50 & $<5$ & $<20$ \\
\hline $42252 b$ & 50 & 1,000 & 4.0 & $N$ & 10 & 70 & 20 & 30 & $N$ & $N$ \\
\hline 422527 & 100 & 1,000 & 11.0 & $N$ & 20 & 100 & 20 & 50 & $N$ & $N$ \\
\hline 422528 & 70 & 1,000 & 11.0 & $N$ & 15 & 100 & 15 & 30 & 10 & $N$ \\
\hline 422529 & 100 & 1,500 & 1.0 & $N$ & 15 & 100 & 20 & 30 & $N$ & $N$ \\
\hline 422530 & 70 & 1,500 & $\langle 1.0$ & $N$ & 10 & 70 & 20 & 30 & $N$ & $N$ \\
\hline
\end{tabular}


TABLE 2.--RESULTS OF SPECTROGRAPHIC AMALYSES OF NURE STREAM-SEDIMEHT SAMPLES FROM THE LIVENGCOD

AND WESTERN $1 / 3$ OF THE CIRCLE DUADRANGLES, ALASKA--ContinuEd

\begin{tabular}{|c|c|c|c|c|c|c|c|c|c|c|c|}
\hline Sample & $\begin{array}{c}\mathrm{Ni}-\mathrm{pp} \mathbb{} \\
5\end{array}$ & 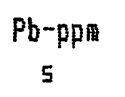 & $\begin{array}{c}\text { Sc-ppl } \\
5\end{array}$ & $\begin{array}{c}5 n-p p m \\
5\end{array}$ & $\begin{array}{c}5 r-p p \\
5\end{array}$ & $\begin{array}{c}V-p p \\
s\end{array}$ & $\begin{array}{c}H-p p p \\
s\end{array}$ & $\begin{array}{c}\text { Y-ppa } \\
5\end{array}$ & $\begin{array}{c}2 n-p p a \\
5\end{array}$ & $\begin{array}{c}2 r-p p l \\
s\end{array}$ & $\begin{array}{c}\text { Th-pp } \\
5\end{array}$ \\
\hline 422486 & 30 & 15 & 15 & $N$ & 200 & 100 & $N$ & 20 & $N$ & 200 & $N$ \\
\hline 422487 & 50 & 20 & 15 & $N$ & 300 & 100 & $N$ & 30 & $N$ & 200 & $N$ \\
\hline 422488 & 15 & 10 & 10 & $H$ & 200 & 100 & $N$ & 20 & $N$ & 200 & $N$ \\
\hline 422489 & 30 & 30 & 15 & $N$ & 200 & 100 & $N$ & 50 & $N$ & 150 & $N$ \\
\hline 422490 & 10 & $N$ & $<5$ & $N$ & $<100$ & 20 & N & $N$ & $N$ & 20 & $N$ \\
\hline 422491 & 30 & 30 & 20 & $N$ & 200 & 100 & $N$ & 30 & $N$ & 150 & $N$ \\
\hline 422492 & 30 & 20 & 15 & $N$ & 200 & 100 & $N$ & 20 & $N$ & 200 & $N$ \\
\hline 422493 & 20 & 20 & 10 & $N$ & 150 & 100 & $N$ & 15 & $N$ & 150 & $N$ \\
\hline 422494 & 30 & 50 & 15 & 10 & 200 & 100 & $N$ & 30 & $N$ & 150 & N \\
\hline 422495 & 30 & 30 & 15 & 10 & 150 & 100 & $N$ & 20 & $N$ & 150 & N \\
\hline 422496 & 30 & 20 & 15 & $N$ & 300 & 100 & $H$ & 20 & $N$ & 200 & N \\
\hline 422497 & 30 & 50 & 20 & $N$ & 300 & 100 & $N$ & 20 & $N$ & 200 & $N$ \\
\hline 422498 & 30 & 20 & 20 & $N$ & 200 & 100 & $N$ & 20 & $N$ & 200 & N \\
\hline 422499 & 30 & 20 & 15 & $N$ & 200 & 100 & $N$ & 20 & $N$ & 200 & $N$ \\
\hline 422500 & 20 & 15 & 15 & $H$ & 200 & 100 & $N$ & 20 & $N$ & 200 & H \\
\hline 422501 & 20 & 20 & 20 & $N$ & 200 & 100 & $N$ & 30 & $N$ & 200 & $N$ \\
\hline 422502 & 30 & 20 & 20 & N & 200 & 100 & $N$ & 30 & $N$ & 200 & $N$ \\
\hline 422503 & 10 & 10 & 7 & $N$ & 100 & 70 & $N$ & 15 & $N$ & 150 & $N$ \\
\hline 422504 & 20 & 30 & 20 & $N$ & 200 & 100 & $N$ & 20 & $N$ & 150 & $N$ \\
\hline 422505 & 50 & 70 & 20 & $N$ & 150 & 100 & N & 30 & $N$ & 300 & $N$ \\
\hline 422506 & 50 & 100 & 15 & N & 100 & 100 & $N$ & 20 & $<200$ & 150 & $N$ \\
\hline 422507 & 50 & 10 & 10 & $N$ & 100 & 70 & $N$ & 20 & $N$ & 200 & $N$ \\
\hline 422508 & 30 & 20 & 20 & N & 200 & 100 & N & 30 & $N$ & 500 & $H$ \\
\hline 422509 & 50 & 70 & 20 & $N$ & 200 & 100 & $N$ & 50 & $N$ & 500 & $N$ \\
\hline 422510 & 70 & 50 & 20 & $N$ & 200 & 100 & $N$ & 50 & $N$ & 500 & N \\
\hline 422511 & 100 & 30 & 20 & $N$ & 200 & 100 & $N$ & 50 & N & 300 & $N$ \\
\hline 422512 & 100 & 30 & 20 & $N$ & 300 & 100 & $N$ & 70 & $N$ & 200 & $N$ \\
\hline 422513 & 100 & 20 & 15 & $N$ & 300 & 100 & $N$ & 50 & $N$ & 200 & $N$ \\
\hline 422514 & 70 & 20 & 20 & $N$ & 200 & 100 & $N$ & 50 & $N$ & 200 & $N$ \\
\hline 422515 & 100 & 30 & 20 & $N$ & 200 & 100 & $N$ & 50 & $N$ & 200 & $N$ \\
\hline 422516 & 70 & 30 & 20 & $N$ & 150 & 100 & $N$ & 50 & $N$ & 200 & $N$ \\
\hline 422517 & 100 & 20 & 20 & $N$ & 200 & 100 & $<50$ & 50 & $N$ & 1,000 & N \\
\hline 422518 & 70 & 70 & 20 & $H$ & 200 & 100 & $H$ & 50 & $N$ & 200 & $N$ \\
\hline 422519 & 100 & 100 & 20 & N & 300 & 100 & $N$ & 70 & $N$ & 500 & $N$ \\
\hline 422520 & 50 & 20 & 20 & N & 200 & 100 & $N$ & 50 & N & 500 & N \\
\hline 422521 & 100 & 50 & 20 & $N$ & 200 & 150 & $N$ & 50 & $<200$ & 300 & $N$ \\
\hline 422522 & 50 & 50 & 15 & $N$ & 200 & 100 & $N$ & 20 & $N$ & 200 & $N$ \\
\hline 422523 & 30 & 30 & 20 & $N$ & 300 & 100 & $N$ & 50 & $N$ & 300 & $N$ \\
\hline 422524 & 20 & 20 & 15 & $N$ & 150 & 100 & $N$ & 30 & $N$ & 150 & $N$ \\
\hline 422525 & 50 & 70 & 20 & $N$ & 200 & 100 & $N$ & 30 & $N$ & 150 & $N$ \\
\hline $42252 b$ & 20 & 20 & 15 & $N$ & 200 & 100 & $N$ & 20 & $N$ & 200 & $N$ \\
\hline 422527 & 30 & 30 & 20 & $N$ & 200 & 100 & $N$ & 30 & $N$ & 200 & N \\
\hline 422528 & 20 & 15 & 15 & N & 150 & 100 & $N$ & 20 & $N$ & 200 & $N$ \\
\hline 422529 & 30 & 30 & 20 & $N$ & 200 & 100 & $N$ & 30 & N & 200 & $N$ \\
\hline 422530 & 20 & 20 & 15 & H & 300 & 100 & $N$ & 20 & $N$ & 200 & $N$ \\
\hline
\end{tabular}


TABLE 2.--RESULTS OF SPECTROGRAFHIC AMALYSES OF NURE STREAM-SEDIMENT SAMPLES FROH THE LIVENGODD

AND WESTERN $1 / 3$ OF THE CIRCLE QUADRANGLES, ALASKA--ContinUEd

\begin{tabular}{|c|c|c|c|c|c|c|c|c|c|}
\hline Sample & Latitude & Longitude & $\begin{array}{c}\text { Fe-pct. } \\
5\end{array}$ & $\begin{array}{c}m_{g}-p c t . \\
5\end{array}$ & $\begin{array}{c}\text { Ca-pct. } \\
5\end{array}$ & $\begin{array}{c}\text { Ti-pct. } \\
\Xi\end{array}$ & $\begin{array}{c}M n-p p n \\
5\end{array}$ & $\begin{array}{c}\mathrm{Ag}-p \rho n \\
\mathrm{~s}\end{array}$ & $\begin{array}{c}\text { As-ppm } \\
5\end{array}$ \\
\hline 422531 & 653858 & 1494949 & 3.00 & 1.50 & 2.0 & .500 & 500 & N & $N$ \\
\hline 422532 & $6540 \quad 4$ & 1494546 & 2.60 & 1.00 & 1.0 & .500 & 500 & $<.5$ & $N$ \\
\hline 422533 & 65417 & $14948 \quad 21$ & 2.00 & 1.00 & .7 & .500 & 500 & $N$ & $N$ \\
\hline 422534 & 654027 & 1495425 & 2.00 & 1.00 & 1.0 & .500 & 1,000 & $N$ & N \\
\hline 422535 & 653722 & 1495021 & 5.00 & 1.50 & 1.5 & .700 & 700 & .5 & $N$ \\
\hline $42253 b$ & $65 \quad 2 b \quad 12$ & 1494148 & 5.00 & 1.50 & 1.5 & .500 & 1,000 & .7 & N \\
\hline 422537 & 653015 & 1492233 & 3.00 & 1.00 & 1.0 & .500 & 300 & $N$ & N \\
\hline 422538 & 652945 & 1492044 & 2.00 & .70 & 1.0 & .500 & 300 & 4.5 & $N$ \\
\hline 422539 & 652927 & 1491546 & 3.00 & 1.00 & 1.0 & .500 & 300 & $N$ & N \\
\hline 422540 & $65 \quad 418$ & 1495856 & 3.00 & 1.00 & 1.0 & .500 & 700 & N & N \\
\hline 422541 & $65 \quad 424$ & 1495546 & 3.00 & 1.50 & 1.0 & .500 & 300 & $N$ & N \\
\hline 422543 & $65 \quad 439$ & 1495331 & 3.00 & 1.50 & 2.0 & .500 & 500 & $<.5$ & N \\
\hline 422544 & $65 \quad 432$ & 1495147 & 5.00 & 1.50 & 2.0 & .300 & 500 & $<.5$ & $N$ \\
\hline 422545 & $65 \quad b \quad 5$ & 1495013 & 5.00 & 1.50 & 2.0 & .500 & 500 & $<.5$ & $N$ \\
\hline 422546 & $\begin{array}{lll}65 & 6 & 7\end{array}$ & 1494859 & 5.00 & 1.00 & 3.0 & .500 & 500 & $N$ & N \\
\hline 422547 & $65 \quad 445$ & 1494923 & 3.00 & 1.00 & 2.0 & .500 & 500 & $N$ & N \\
\hline 422548 & 6548 & $149 \quad 4717$ & 5.00 & 1.00 & 1.5 & .500 & 500 & $<.5$ & N \\
\hline 422549 & 65357 & 1494353 & 5.00 & 1.00 & 2.0 & .500 & 500 & $<.5$ & $N$ \\
\hline 422550 & 65315 & 1494238 & 5.00 & 1.50 & 2.0 & .500 & 500 & $<.5$ & N \\
\hline 422551 & 65238 & 1494238 & 7.00 & 1.50 & 2.0 & .500 & 500 & $N$ & $N$ \\
\hline 422552 & $65 \quad 023$ & 1494158 & 5.00 & 1.50 & 2.0 & .500 & 1,000 & $\mathrm{H}$ & $N$ \\
\hline 422553 & $65 \quad 059$ & 149418 & 2.00 & .50 & .7 & .070 & 200 & $N$ & $N$ \\
\hline 422554 & 65025 & $14940 \quad 0$ & 5.00 & 1.50 & 1.0 & .500 & 200 & 2.0 & $N$ \\
\hline 422555 & 65050 & $14938 \quad 2$ & 5.00 & 2.00 & 1.0 & .500 & 500 & $<.5$ & N \\
\hline 422556 & $65 \quad 120$ & 1493620 & 1.00 & .70 & .5 & .150 & 200 & $\mathrm{H}$ & $N$ \\
\hline 422557 & $65 \quad 145$ & 1493356 & 3.00 & 1.00 & 1.0 & .500 & 300 & $<.5$ & $N$ \\
\hline 422559 & $65 \quad 244$ & 1493030 & 5.00 & 1.50 & 1.0 & .500 & 500 & $<.5$ & $N$ \\
\hline 422559 & 65339 & $14933 \quad 5$ & 1.50 & .70 & .7 & .150 & 200 & $N$ & $N$ \\
\hline 422561 & 65311 & 1493545 & 2.00 & 1.00 & 1.0 & .200 & 300 & $N$ & N \\
\hline 422562 & $\begin{array}{lll}65 & b & 7\end{array}$ & $149442 b$ & 3.00 & 1.00 & 1.0 & .300 & 500 & N & $N$ \\
\hline 422563 & $65 \quad 620$ & $14946 \quad 10$ & 5.00 & 1.50 & 1.0 & .500 & 500 & 6.5 & $N$ \\
\hline 422564 & 651032 & 1493151 & 3.00 & .70 & .7 & .200 & 200 & N & $N$ \\
\hline 422565 & 65821 & 1492854 & 1.00 & .70 & .7 & .150 & 200 & $N$ & $N$ \\
\hline 422566 & 65839 & 1492638 & 1.50 & .70 & .7 & .200 & 200 & $N$ & $N$ \\
\hline 422567 & $65 \quad 553$ & 1493220 & .20 & .05 & .2 & .050 & 100 & $N$ & $N$ \\
\hline 422568 & $b 5 \quad 5 \quad 17$ & 1492944 & 1.50 & .70 & .3 & .150 & 200 & $N$ & N \\
\hline 422569 & $65 \quad 517$ & 1492526 & 1.50 & 1.00 & .7 & .200 & 200 & $N$ & $N$ \\
\hline 422571 & $65 \quad 557$ & $14924 \quad 6$ & 1.50 & .70 & .5 & .150 & 150 & N & $N$ \\
\hline 422572 & $65 \quad 633$ & 149215 & 2.00 & 1.00 & .5 & .200 & 200 & N & N \\
\hline 422573 & $65 \quad 843$ & 1491941 & 1.50 & 1.00 & .5 & .150 & 200 & $N$ & $N$ \\
\hline 422574 & 65938 & 1491938 & 1.50 & 1.00 & .5 & .200 & 300 & $N$ & $N$ \\
\hline 422575 & 651049 & 1491946 & 2.00 & .70 & .5 & .200 & 200 & $N$ & $N$ \\
\hline 422576 & 651115 & 1492259 & 1.50 & .70 & .5 & .150 & 500 & $N$ & $N$ \\
\hline 422577 & 651152 & 149118 & 1.50 & .70 & .5 & .200 & 150 & N & $N$ \\
\hline 422578 & 651129 & 1491351 & 1.50 & .70 & .5 & .200 & 200 & $N$ & $N$ \\
\hline
\end{tabular}


TABLE 2,--RESULTS OF SPECTROGRAPHIC ANALYSES OF NURE STREAM-SEDIMENT SAMPLES FROM THE LIVEMG00D

AND WESTERN $1 / 3$, OF THE CIRCLE QUADRANGLES, ALASKA--Continued

\begin{tabular}{|c|c|c|c|c|c|c|c|c|c|c|}
\hline Sample & $\begin{array}{c}\text { B-ppi } \\
5\end{array}$ & $\begin{array}{c}\text { Ba-ppA } \\
5\end{array}$ & $\begin{array}{c}\text { Be-pp } \\
5\end{array}$ & $\begin{array}{c}\text { Bi-ppo } \\
5\end{array}$ & $\begin{array}{c}\text { Co-pp } \\
5\end{array}$ & $\begin{array}{c}\text { Cr-ppa } \\
5\end{array}$ & $\begin{array}{c}\text { Cu-ppi } \\
5\end{array}$ & $\begin{array}{l}\text { La-ppi } \\
5\end{array}$ & $\begin{array}{c}\text { Mo-ppm } \\
5\end{array}$ & $\begin{array}{c}N b-p p a \\
5\end{array}$ \\
\hline 422531 & 70 & 1,500 & 1.0 & $H$ & 15 & 100 & 20 & 30 & $N$ & $N$ \\
\hline 422532 & 70 & 1,500 & $\langle 1.0$ & $N$ & 15 & 100 & 20 & 30 & $N$ & $N$ \\
\hline 422533 & 100 & 2,000 & $\langle 1.0$ & $N$ & 15 & 150 & 20 & 50 & $<5$ & $N$ \\
\hline 422534 & 100 & 2,000 & 11.0 & $N$ & 20 & 150 & 20 & 50 & 15 & $N$ \\
\hline 422535 & 100 & 1,500 & 1.0 & $N$ & 20 & 100 & 20 & 70 & $N$ & $<20$ \\
\hline 422536 & 70 & 2,000 & 41.0 & $\mathrm{~N}$ & 20 & 200 & 20 & 70 & $N$ & $H$ \\
\hline 422537 & 70 & 1,500 & 4.0 & $N$ & 20 & 100 & 20 & 50 & H & $N$ \\
\hline 422538 & 70 & 1,000 & $\langle 1.0$ & $N$ & 10 & 50 & 15 & 20 & $N$ & $N$ \\
\hline 422539 & 70 & 1,000 & $\langle 1.0$ & $N$ & 15 & 150 & 15 & 50 & $N$ & $N$ \\
\hline 422540 & 70 & 1,000 & 01.0 & $N$ & 15 & 70 & 15 & 30 & $N$ & $N$ \\
\hline 422541 & 70 & 1,500 & $\langle 1.0$ & $N$ & 15 & 100 & 20 & 30 & $<5$ & $N$ \\
\hline 422543 & 70 & 1,500 & $N$ & $N$ & 10 & 100 & 20 & 30 & 10 & $N$ \\
\hline 422544 & 70 & 1,500 & $\langle 1.0$ & $N$ & 15 & 100 & 20 & 30 & $H$ & $N$ \\
\hline 422545 & 100 & 1,500 & $\langle 1.0$ & $N$ & 15 & 70 & 30 & 50 & $N$ & $N$ \\
\hline 422546 & 100 & 1,500 & $\$ 1.0$ & $N$ & 15 & 70 & 20 & 50 & $\langle 5$ & $N$ \\
\hline 422547 & 50 & 700 & $\langle 1.0$ & $N$ & 10 & 100 & 20 & 30 & $N$ & $N$ \\
\hline 422548 & 70 & 1,000 & $\langle 1.0$ & $N$ & 15 & 100 & 20 & 30 & $N$ & $N$ \\
\hline 422549 & 70 & 1,500 & $\langle 1.0$ & $N$ & 15 & 100 & 20 & 50 & $N$ & $N$ \\
\hline 422550 & 70 & 1,500 & $\$ 1.0$ & $N$ & 15 & 100 & 15 & 50 & $\ddot{N}$ & $N$ \\
\hline 422551 & 70 & 1,500 & $\langle 1.0$ & $N$ & 15 & 100 & 50 & 30 & $N$ & $N$ \\
\hline 422552 & 100 & 1,500 & $\langle 1.0$ & $N$ & 20 & 100 & 20 & 50 & $N$ & $N$ \\
\hline 422553 & 20 & 500 & $\langle 1.0$ & $N$ & $N$ & 20 & 15 & $N$ & $N$ & $N$ \\
\hline 422554 & 70 & 1,000 & $\langle 1.0$ & $N$ & 15 & 100 & 30 & 50 & $N$ & $N$ \\
\hline 422555 & 50 & 1,500 & $\langle 1.0$ & $N$ & 20 & 100 & 30 & 50 & $N$ & $N$ \\
\hline 422556 & 20 & 500 & $\langle 1.0$ & $N$ & $N$ & 30 & 15 & $N$ & $N$ & $N$ \\
\hline 422557 & 100 & 1,500 & $\langle 1.0$ & $N$ & 10 & 100 & 20 & 50 & 5 & $N$ \\
\hline 422558 & 50 & 1,000 & $\langle 1.0$ & $N$ & 15 & 50 & 30 & 30 & 5 & $N$ \\
\hline 422559 & 20 & 700 & $\langle 1.0$ & $N$ & $\langle 5$ & 20 & 15 & $<20$ & $N$ & $N$ \\
\hline 422561 & 50 & 1,000 & $\langle 1.0$ & $N$ & 10 & 30 & 15 & 20 & 5 & $N$ \\
\hline 422562 & 50 & 1,500 & $<1.0$ & $N$ & 10 & 100 & 20 & 50 & $N$ & $H$ \\
\hline 422563 & 70 & 1,500 & $\langle 1.0$ & $N$ & 15 & 200 & 30 & 30 & $<5$ & $N$ \\
\hline 422564 & 30 & 500 & $\langle 1.0$ & $N$ & 10 & 50 & 15 & 30 & $N$ & $N$ \\
\hline 422565 & 20 & 300 & 41,0 & $N$ & 7 & 50 & 15 & 30 & $N$ & $N$ \\
\hline 422566 & 20 & 500 & $<1.0$ & $N$ & 10 & 50 & 10 & 50 & $\langle 5$ & $N$ \\
\hline 422567 & $N$ & 100 & 41.0 & $N$ & $N$ & 10 & 5 & $N$ & $H$ & $N$ \\
\hline 422569 & 50 & 300 & $\langle 1.0$ & $N$ & 10 & 50 & 15 & 30 & $N$ & $N$ \\
\hline 422569 & 20 & 500 & $\langle 1.0$ & $N$ & 10 & 50 & 20 & 20 & $N$ & $N$ \\
\hline 422571 & 20 & 500 & 11.0 & $N$ & 10 & 50 & 15 & 30 & $N$ & $N$ \\
\hline 422572 & 15 & 500 & 31.0 & $\mathrm{~N}$ & 10 & 50 & 20 & 20 & N & N \\
\hline 422573 & 20 & 500 & $\langle 1.0$ & $N$ & 15 & 50 & 20 & 30 & $N$ & $N$ \\
\hline 422574 & 30 & 500 & 11.0 & $N$ & 15 & 30 & 15 & 30 & $N$ & $N$ \\
\hline 422575 & 20 & 500 & 1.0 & $N$ & 10 & 30 & 20 & $<20$ & N & H \\
\hline 422576 & 20 & 500 & $\langle 1.0$ & $N$ & 10 & 20 & 15 & 30 & $H$ & $N$ \\
\hline 422577 & 30 & 500 & $\langle 1.0$ & $N$ & 10 & 50 & 20 & 30 & N & N \\
\hline 422578 & 50 & 500 & $\langle 1.0$ & $\mathrm{N}$ & 10 & 50 & 20 & 30 & $N$ & $N$ \\
\hline
\end{tabular}


TABLE 2.--RESULTS OF SPECTROGRAPHIC ANALYSES OF NURE STREAM-SEDIMENT SAMPLES FROM THE LIVENGOOD

AND HESTERN $1 / 3$ OF THE CIRCLE QUADRAMGLES, ALASKA--Continued

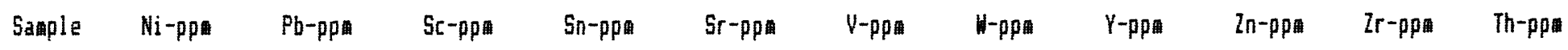

s $5 \quad 5 \quad s$

$\mathbf{s}$

s

$\begin{array}{lll}N & 300 & 100 \\ N & 200 & 100\end{array}$

$\begin{array}{llll}422531 & 30 & 20 & 20 \\ 422532 & 30 & 20 & 20\end{array}$

$200 \quad 100$

$422533 \quad 30$

$20 \quad 20$

$422534 \quad 30$

$20 \quad 20$

200

100

$422535 \quad 20$

$30 \quad 20$

$300 \quad 100$

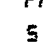

5

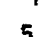

5

5

$\begin{array}{llll}422536 & 20 & 50 & 20 \\ 422537 & 30 & 20 & 20 \\ 422538 & 30 & 15 & 15 \\ 422539 & 15 & 20 & 15 \\ 422540 & 30 & 20 & 15\end{array}$

$300 \quad 100$

$200 \quad 100$

150

200

100

100

$200 \quad 100$

$\begin{array}{ll}N & 30 \\ N & 30 \\ N & 20 \\ N & 30 \\ N & 30\end{array}$

$\begin{array}{ll}N & 200 \\ N & 300 \\ N & 200 \\ N & 500 \\ N & 300\end{array}$

N 20

N 20

N 200

N 20

N 200

5

\section{1}

$20 \quad 20 \quad 20$

$\begin{array}{ll}20 & 20 \\ 20 & 10\end{array}$

422543
422544

30

$20 \quad 20$

$422545 \quad 30$

$50 \quad 20$

$42254 b \quad 50$

$20 \quad 20$

$422547 \quad 20$

$15 \quad 15$

$\begin{array}{ll}422548 & 30 \\ 422549 & 30\end{array}$

$30 \quad 20$

422550

422551

$15 \quad 20$

$30 \quad 20$

$\begin{array}{ll}422552 & 50 \\ 422553 & 10 \\ 422554 & 30 \\ 422555 & 30 \\ 422556 & 10\end{array}$

$30 \quad 20$

\section{$200 \quad 100$}

$200 \quad 100$

$200 \quad 100$

200

100

$300 \quad 100$

$\begin{array}{ll}N & 20 \\ N & 20\end{array}$

H 200

N 300 
TABLE 2,--RESULTS OF SPECTROGRAPHIC ANALYSES OF NURE STREAM-SEDIMENT SAMPLES FROH THE LIVENGOOD AND WESTERN $1 / 3$ OF THE CIRCLE QUADRANGLES, ALASKA--Continued

\begin{tabular}{|c|c|c|c|c|c|c|c|c|c|}
\hline Sample & Latitude & Longitude & $\begin{array}{c}\text { Fe-pct. } \\
5\end{array}$ & $\begin{array}{c}\mathrm{Mg}-p c t . \\
s\end{array}$ & $\begin{array}{c}\text { Ca-pct. } \\
5\end{array}$ & $\begin{array}{c}\text { Ti-pct. } \\
5\end{array}$ & $\begin{array}{c}\text { Mn-ppu } \\
5\end{array}$ & $\begin{array}{c}\text { Ag-ppu } \\
5\end{array}$ & $\begin{array}{c}\text { As-ppn } \\
5\end{array}$ \\
\hline 422579 & 651055 & 1491655 & 1.00 & .50 & .5 & .200 & 200 & $N$ & $N$ \\
\hline 422580 & 651018 & $14914 \quad 8$ & 1.00 & .50 & .5 & .200 & 150 & $N$ & $N$ \\
\hline 422581 & 65951 & 1491345 & 1.00 & .70 & .5 & .200 & 150 & $N$ & $N$ \\
\hline 422582 & 653815 & 1495626 & 1.00 & .30 & .5 & .100 & 200 & N & N \\
\hline 422583 & $6546 \quad 14$ & 1495224 & 1.50 & 1.00 & .5 & .200 & 150 & $N$ & $N$ \\
\hline 422584 & 654622 & $14955 \quad 23$ & 1.00 & 1.00 & .5 & .200 & 200 & $N$ & $N$ \\
\hline 422585 & 651859 & 149128 & 5.00 & 1.50 & 2.0 & .700 & 700 & $N$ & $N$ \\
\hline 422586 & 651959 & 1491717 & 5.00 & 1.50 & 3.0 & .700 & 1,000 & $<.5$ & $N$ \\
\hline 422587 & 651429 & 1485053 & 5.00 & 1.50 & 3.0 & .700 & 700 & $N$ & $N$ \\
\hline 422588 & $6514 \quad 4$ & 14910 & 7.00 & 2.00 & 3.0 & 1.000 & 700 & $N$ & $N$ \\
\hline 422589 & 651412 & $149 \quad 4 \quad 32$ & 5.00 & 2.00 & 3.0 & .700 & 1,000 & N & $N$ \\
\hline 422590 & 651359 & 14976 & 7.00 & 2.00 & 3.0 & 1.000 & 1,000 & $N$ & $N$ \\
\hline 422591 & $65 \quad 0 \quad 42$ & $148 \div 20$ & 5.00 & 1.50 & 1.5 & .700 & 1,000 & $N$ & $N$ \\
\hline 422592 & $65 \quad 235$ & $148 \quad 15 \quad 24$ & 7.00 & 2.00 & 3.0 & 1.000 & 1,000 & $N$ & $N$ \\
\hline 422593 & $65 b 6$ & 1481820 & 5.00 & 1.50 & 2.0 & .500 & 500 & $N$ & $N$ \\
\hline 422594 & 6542 & $148 \quad 24 \quad 42$ & 7.00 & 2.00 & 3.0 & 1.000 & 1,000 & N & $N$ \\
\hline 422596 & 65356 & $148 \quad 28 \quad 27$ & 10.00 & 3.00 & 3.0 & 1.000 & 1,500 & $N$ & $N$ \\
\hline 422597 & 651313 & 1495926 & 2.00 & 1.50 & 2.0 & .500 & 500 & $N$ & $N$ \\
\hline 422598 & 651328 & 149512 & 7.00 & 2.00 & 2.0 & .700 & 700 & N & $N$ \\
\hline 422599 & 651248 & 1494712 & 7.00 & 2.00 & 2.0 & .700 & 1,000 & $N$ & $N$ \\
\hline 422600 & 651650 & 1494254 & 10.00 & 2.00 & 3.0 & 1.000 & 700 & N & $\mathrm{H}$ \\
\hline 422601 & 651832 & 1494230 & 7.00 & 2.00 & 2.0 & .700 & 1,000 & $N$ & $N$ \\
\hline 422602 & 651932 & 1493937 & 7.00 & 2.00 & 2.0 & 1.000 & 700 & $N$ & $N$ \\
\hline 422603 & 65220 & 1495121 & 10.00 & 2.00 & 2.0 & .700 & 1,000 & $N$ & $N$ \\
\hline 422604 & 652155 & 1495456 & 5.00 & 1.50 & 1.0 & .500 & 700 & N & $N$ \\
\hline 422605 & 652329 & $14955 \quad 2$ & 5.00 & 1.50 & 1.5 & .500 & 700 & $N$ & $N$ \\
\hline 422606 & 65241 & $149 \quad 4852$ & 5.00 & 1.50 & 2.0 & 1.000 & 700 & 6.5 & $N$ \\
\hline 422607 & 65281 & 1495030 & 5.00 & 2.00 & 3.0 & 1.000 & 700 & $N$ & $N$ \\
\hline 422608 & 652824 & 1495353 & 7.00 & 2.00 & 3.0 & 1.000 & 1,000 & N & $N$ \\
\hline 422609 & 65289 & $14958 \quad 45$ & 5.00 & 1.50 & 2.0 & .700 & 700 & $N$ & $N$ \\
\hline 422610 & 652824 & 149590 & 7.00 & 1.50 & 2.0 & 1.000 & 700 & $N$ & N \\
\hline 422611 & 655015 & 1491823 & 7.00 & 1.50 & 2.0 & 1.000 & 700 & $<.5$ & N \\
\hline $422 b 12$ & 654927 & 1491154 & 3.00 & 1.50 & 1.0 & .500 & 700 & $N$ & $N$ \\
\hline 422613 & 654924 & $149 \quad 121$ & 7.00 & 1.50 & 2.0 & 1.000 & 1,000 & $N$ & $N$ \\
\hline 422614 & 654757 & 1493141 & 7.00 & 1.50 & 2.0 & 1.000 & 700 & $N$ & $N$ \\
\hline 422615 & 654542 & $149 \quad 45 \quad 17$ & 7.00 & 1.50 & 3.0 & 1.000 & 1,000 & $N$ & $N$ \\
\hline 422616 & $65 \quad 4632$ & 1495845 & 2.00 & .70 & 1.5 & .500 & 300 & $N$ & $N$ \\
\hline 422617 & $65 \quad 857$ & $149 \quad 1358$ & 7.00 & 2.00 & 3.0 & 1.000 & 1,000 & $\mu$ & $N$ \\
\hline 422618 & 65750 & 1491541 & 5.00 & 1.50 & 2.0 & .700 & 500 & $N$ & $N$ \\
\hline 422619 & $65 \quad b 11$ & 1491732 & 7.00 & 2.00 & 2.0 & 1.000 & 700 & $N$ & $N$ \\
\hline 422620 & $65 \quad b \quad 7$ & 1491341 & 10.00 & 2.00 & 2.0 & 1.000 & 700 & $N$ & N \\
\hline 422621 & $65 \quad 521$ & 1491510 & 7.00 & 2.00 & 3.0 & 1.000 & 1,000 & $N$ & $N$ \\
\hline 422622 & 65453 & 1491917 & 7.00 & 2.00 & 2.0 & 1.000 & 1,000 & $N$ & N \\
\hline 422623 & 65346 & 1492059 & 7.00 & 2.00 & 2.0 & 1.000 & 700 & $\langle .5$ & $N$ \\
\hline 422624 & 65315 & 149233 & 7.00 & 2.00 & 3.0 & 1.000 & 1,000 & $N$ & N \\
\hline
\end{tabular}


TABLE 2.--RESULTS OF SPECTROGRAPHIC ANALYSES OF NURE STREAM-SEDIMENT SAMPLES FFOK THE LIVENGOOD

AND WESTERN $1 / 3$ OF THE CIRCLE QUADFAMGLES, ALASKA--Continued

\begin{tabular}{|c|c|c|c|c|c|c|c|c|c|c|}
\hline Sáple & $\begin{array}{c}\text { B-ppoi } \\
5\end{array}$ & $\begin{array}{c}\text { Ba-pp而 } \\
5\end{array}$ & $\begin{array}{c}\text { Be-ppin } \\
5\end{array}$ & $\begin{array}{c}\text { Bi-pp解 } \\
5\end{array}$ & $\begin{array}{c}\text { [o-ppon } \\
5\end{array}$ & $\begin{array}{c}\text { Cr-pprit } \\
s\end{array}$ & $\begin{array}{c}\text { Cu-ppi } \\
s\end{array}$ & $\begin{array}{l}\text { La-pp面 } \\
5\end{array}$ & $\begin{array}{c}\text { Mo-ppi } \\
5\end{array}$ & $\begin{array}{c}\text { Hb-ppo } \\
5\end{array}$ \\
\hline 422579 & 30 & 500 & 41.0 & $N$ & 10 & 50 & 10 & 50 & $N$ & $N$ \\
\hline 422590 & 30 & 500 & $\langle 1.0$ & $N$ & 10 & 30 & 10 & 20 & $N$ & $N$ \\
\hline 422581 & 30 & 500 & 41.0 & $N$ & 10 & 50 & 15 & 30 & $<5$ & $N$ \\
\hline 422592 & 15 & 500 & $\langle 1.0$ & $N$ & 10 & 20 & 20 & 20 & $N$ & $N$ \\
\hline 422583 & 20 & 500 & 41.0 & $N$ & 10 & 50 & 15 & 30 & $N$ & $N$ \\
\hline 422584 & 30 & 500 & $\$ 1.0$ & $N$ & 15 & 50 & 20 & 20 & $N$ & $N$ \\
\hline 422585 & 30 & 1,000 & 1.0 & $N$ & 10 & 70 & 30 & 20 & $N$ & $N$ \\
\hline 422586 & 50 & 1,000 & 1.0 & $N$ & 20 & 100 & 50 & 20 & $\langle 5$ & $N$ \\
\hline 422587 & 50 & 1,500 & 1.0 & $N$ & 15 & 100 & 50 & 30 & $N$ & $N$ \\
\hline 422589 & 70 & 1,500 & $\langle 1.0$ & $N$ & 30 & 200 & 70 & 50 & $N$ & $N$ \\
\hline 422589 & 50 & 1,500 & 1.0 & $N$ & 20 & 150 & 70 & 30 & $N$ & H \\
\hline 422590 & 50 & 1,500 & 1.0 & $N$ & 15 & 150 & 50 & 50 & $N$ & $N$ \\
\hline 422591 & 70 & 1,000 & 1.0 & $N$ & 15 & 100 & 20 & 30 & $N$ & $N$ \\
\hline 422592 & 70 & 1,500 & $\langle 1.0$ & $N$ & 20 & 200 & 50 & 50 & $N$ & $<20$ \\
\hline 422593 & 50 & 1,000 & 1.0 & $N$ & 15 & 150 & 50 & 30 & $<5$ & $N$ \\
\hline 422594 & 70 & 2,000 & 1.0 & $N$ & 15 & 200 & 50 & 50 & $N$ & $N$ \\
\hline 422596 & 70 & 2,000 & 1.0 & $N$ & 30 & 200 & 70 & 50 & $N$ & $<20$ \\
\hline 422597 & 30 & 700 & 1.0 & $N$ & 7 & 50 & 15 & $N$ & $N$ & $N$ \\
\hline 422599 & 70 & 1,500 & 1.0 & $N$ & 15 & 150 & 50 & 30 & $N$ & $N$ \\
\hline 422599 & 70 & 1,500 & 1.0 & $N$ & 15 & 150 & 70 & 30 & $N$ & $N$ \\
\hline 422600 & 70 & 2,000 & 1.0 & $N$ & 15 & 200 & 70 & 70 & $N$ & 20 \\
\hline 422601 & 50 & 2,000 & 1.0 & $N$ & 20 & 150 & 70 & 50 & $\langle 5$ & $<20$ \\
\hline 422602 & 50 & 1,500 & 1.0 & $N$ & 20 & 150 & 70 & 50 & $N$ & $<20$ \\
\hline 422603 & 70 & 1,500 & 1.0 & $N$ & 20 & 200 & 70 & 30 & $N$ & $N$ \\
\hline 422604 & 30 & 1,000 & 1.0 & $N$ & 10 & 100 & 50 & $N$ & $N$ & $N$ \\
\hline 422605 & 50 & 1,000 & 1.0 & $N$ & 10 & 100 & 30 & $N$ & $N$ & $N$ \\
\hline 422606 & 70 & 1,500 & 1.0 & $N$ & 20 & 150 & 70 & 50 & $\langle 5$ & $<20$ \\
\hline 422607 & 70 & 1,500 & 1.0 & $N$ & 20 & 100 & 50 & 50 & $N$ & $<20$ \\
\hline 422609 & 70 & 2,000 & 1.0 & $N$ & 20 & 200 & 70 & 50 & $N$ & $<20$ \\
\hline 422609 & 50 & 1,500 & 1.5 & $N$ & 15 & 100 & 50 & 30 & $N$ & $N$ \\
\hline 422610 & 70 & 1,500 & 1.0 & $N$ & 30 & 150 & 50 & 30 & $N$ & $N$ \\
\hline 422611 & 70 & 2,000 & 1.0 & $N$ & 20 & 150 & 50 & 50 & $\mathrm{~N}$ & $<20$ \\
\hline 422612 & 50 & 1,000 & 1.5 & $N$ & 10 & 50 & 30 & $N$ & $N$ & $N$ \\
\hline 422613 & 100 & 2,000 & 1.0 & $N$ & 15 & 150 & 50 & 50 & $N$ & $<20$ \\
\hline 422614 & 100 & 2,000 & 1.0 & $N$ & 15 & 150 & 20 & 30 & $N$ & 60 \\
\hline 422615 & 70 & 2,000 & $\langle 1.0$ & $N$ & 20 & 100 & 50 & 50 & $N$ & $<20$ \\
\hline 422616 & 20 & 700 & 1.0 & $N$ & 5 & 30 & 10 & $N$ & $N$ & $N$ \\
\hline 422617 & 100 & 2,000 & 11.0 & $N$ & 15 & 200 & 30 & 30 & $N$ & $<20$ \\
\hline 422618 & 70 & 1,500 & $<1.0$ & $N$ & 10 & 200 & 30 & 30 & $N$ & $N$ \\
\hline 422619 & 70 & 2,000 & 1.0 & $N$ & 15 & 200 & 50 & 50 & $N$ & 20 \\
\hline 422620 & 100 & 2,000 & 41.0 & $N$ & 20 & 200 & 70 & 50 & $N$ & $<20$ \\
\hline 422621 & 100 & 2,000 & $(1.0$ & $N$ & 20 & 200 & 70 & 50 & $N$ & 20 \\
\hline 422622 & 100 & 2,000 & 1.0 & $N$ & 20 & 150 & 50 & 50 & $N$ & $<20$ \\
\hline 422623 & 100 & 2,000 & $\langle 1.0$ & $N$ & 30 & 200 & 70 & 50 & $N$ & $N$ \\
\hline 422624 & 70 & 2,000 & $\langle 1.0$ & $N$ & 20 & 200 & 70 & 50 & $N$ & $N$ \\
\hline
\end{tabular}


TABLE 2.--RESULTS OF SPECTROGRAPHIC AMALYSES OF NURE STREAM-SEDIMENT SAMPLES FROM THE LIVENGODD AND HESTERN $1 / 3$ OF THE CIRCLE QUAARRANGLES, ALASKA--Continued

\begin{tabular}{|c|c|c|c|c|c|c|c|c|c|c|c|}
\hline Sample & $\begin{array}{c}\mathrm{Ni}-\mathrm{pp} \text { 相 } \\
5\end{array}$ & $\begin{array}{c}\mathrm{Pb}-\mathrm{pp} \text { i } \\
5\end{array}$ & $\begin{array}{c}\text { Sc-pp而 } \\
5\end{array}$ & $\begin{array}{c}\text { Sn-pp冏 } \\
5\end{array}$ & $\begin{array}{c}\text { Sr-pp酉 } \\
5\end{array}$ & $\begin{array}{c}\text { V-ppof } \\
5\end{array}$ & $\begin{array}{c}W-p p \text { 像 } \\
5\end{array}$ & $\begin{array}{c}y \text {-pp鱼 } \\
5\end{array}$ & $\begin{array}{c}\text { Zn-ppm } \\
5\end{array}$ & $\begin{array}{c}2 r \text {-pp丽 } \\
5\end{array}$ & $\begin{array}{c}\text { Th-pp慒 } \\
5\end{array}$ \\
\hline 422579 & 20 & $N$ & 10 & $N$ & 100 & 100 & $N$ & 20 & $N$ & 100 & N \\
\hline 422580 & 20 & $<10$ & 10 & $N$ & 100 & 70 & $N$ & 15 & $N$ & 100 & $N$ \\
\hline 422581 & 20 & 10 & 10 & $N$ & 100 & 70 & $N$ & 20 & $N$ & 150 & N \\
\hline 422582 & 20 & $N$ & 5 & $N$ & $\langle 100$ & 50 & $N$ & 10 & $N$ & 50 & N \\
\hline $4225 B 3$ & 20 & 10 & 10 & $N$ & 150 & 70 & $N$ & 15 & $N$ & 100 & $N$ \\
\hline 422584 & 20 & 10 & 10 & $N$ & 100 & 100 & $N$ & 15 & $N$ & 70 & $N$ \\
\hline 422585 & 50 & 20 & 10 & $N$ & 200 & 150 & $N$ & 30 & $N$ & 150 & $N$ \\
\hline 422586 & 50 & 20 & 15 & $N$ & 200 & 150 & $N$ & 30 & $N$ & 150 & N \\
\hline 422587 & 50 & 20 & 15 & $N$ & 200 & 150 & N & 30 & $N$ & 150 & $N$ \\
\hline 422588 & 50 & 20 & 20 & $N$ & 200 & 200 & $N$ & 50 & $N$ & 200 & $N$ \\
\hline 422589 & 50 & 20 & 20 & $N$ & 200 & 150 & N & 30 & $N$ & 150 & $N$ \\
\hline 422590 & 50 & 30 & 15 & $N$ & 300 & 200 & $N$ & 50 & N & 300 & $N$ \\
\hline 422591 & 30 & 15 & 15 & $N$ & 200 & 150 & $N$ & 30 & $N$ & 200 & $N$ \\
\hline 422592 & 30 & 30 & 20 & $N$ & 300 & 200 & $N$ & 50 & $N$ & 300 & $N$ \\
\hline 422593 & 70 & 20 & 15 & $N$ & 200 & 150 & $N$ & 30 & N & 150 & $N$ \\
\hline 422594 & 30 & 30 & 20 & $N$ & 300 & 200 & N & 30 & $<200$ & 300 & $N$ \\
\hline 422596 & 70 & 50 & 30 & $N$ & 300 & 200 & N & 50 & N & 150 & N \\
\hline 422597 & 20 & 15 & 10 & $N$ & 150 & 150 & N & 20 & $N$ & 150 & $N$ \\
\hline 422598 & 30 & 20 & 20 & $H$ & 300 & 150 & $N$ & 30 & N & 150 & N \\
\hline 422599 & 50 & 50 & 20 & $N$ & 200 & 200 & $N$ & 50 & $N$ & 200 & $N$ \\
\hline 422600 & 50 & 30 & 30 & $H$ & 300 & 200 & $N$ & 50 & $N$ & 300 & $N$ \\
\hline 422601 & 50 & 30 & 20 & $N$ & 200 & 200 & $N$ & 30 & $N$ & 200 & $N$ \\
\hline 422602 & 50 & 30 & 20 & $H$ & 300 & 200 & $N$ & 30 & $N$ & 200 & $N$ \\
\hline 42260.3 & 50 & 30 & 20 & $N$ & 300 & 150 & $N$ & 30 & $N$ & 150 & $N$ \\
\hline 422604 & 50 & 20 & 15 & $N$ & 100 & 150 & N & 20 & $N$ & 150 & N \\
\hline 422605 & 30 & 15 & 15 & $N$ & 200 & 150 & $N$ & 20 & $N$ & 150 & N \\
\hline $42260 \mathrm{~b}$ & 50 & 20 & 15 & $N$ & 200 & 150 & $N$ & 30 & 200 & 200 & N \\
\hline 422607 & 50 & 30 & 20 & $N$ & 200 & 150 & $N$ & 30 & $N$ & 200 & $N$ \\
\hline 422600 & 50 & 50 & 30 & $N$ & 300 & 200 & $N$ & 50 & $N$ & 200 & $H$ \\
\hline 422609 & 50 & 20 & 15 & $N$ & 200 & 150 & $N$ & 30 & $N$ & 150 & $N$ \\
\hline 422610 & 70 & 30 & 20 & $N$ & 200 & 200 & $N$ & 30 & $N$ & 200 & $N$ \\
\hline 422611 & 70 & 30 & 20 & $N$ & 200 & 200 & $N$ & 50 & $N$ & 200 & N \\
\hline 422612 & 50 & 20 & 10 & $\mathbf{N}$ & 100 & 150 & $N$ & 20 & $N$ & 100 & N \\
\hline 422613 & 50 & 30 & 30 & $N$ & 200 & 200 & $N$ & 50 & $N$ & 300 & $N$ \\
\hline 422614 & 30 & 30 & 20 & $N$ & 200 & 200 & $N$ & 30 & $N$ & 300 & $N$ \\
\hline 422615 & 30 & 30 & 20 & $N$ & 200 & 200 & $N$ & 50 & $N$ & 300 & $N$ \\
\hline 422616 & 20 & 10 & 10 & $N$ & 100 & 100 & $\mathrm{~N}$ & 20 & N & 100 & N \\
\hline 422617 & 50 & 20 & 20 & $N$ & 300 & 200 & $N$ & 50 & $N$ & 500 & $N$ \\
\hline 422618 & 30 & 20 & 20 & $N$ & 200 & 200 & $\mathbb{N}$ & 30 & $N$ & 300 & $N$ \\
\hline 422619 & 50 & 30 & 20 & $N$ & 300 & 300 & $N$ & 50 & $<200$ & 500 & $N$ \\
\hline 422620 & 50 & 50 & 30 & $N$ & 300 & 200 & $N$ & 50 & $N$ & 300 & $N$ \\
\hline 422621 & 50 & 30 & 30 & $N$ & 300 & 200 & $N$ & 50 & N & 500 & N \\
\hline 422622 & 50 & 20 & 30 & $N$ & 200 & 200 & $N$ & 50 & $N$ & 300 & $N$ \\
\hline 422623 & 50 & 30 & 20 & $N$ & 300 & 200 & $N$ & 50 & N & 200 & $\mathrm{~N}$ \\
\hline $422 b 24$ & 50 & 20 & 20 & $N$ & 300 & 200 & $N$ & 50 & $N$ & 200 & $N$ \\
\hline
\end{tabular}


TABLE 2. --RESULTS OF SPECTROGRAFHIC ANALYSES OF NURE STREAM-SEDIMENT SAMPLES FROM THE LIVENGOOD AND HESTERN $1 / 3$ OF THE CIRCLE QUADRANGLES, ALASKA--ContinUEd

\begin{tabular}{|c|c|c|c|c|c|c|c|c|c|}
\hline Sample & Latitude & Longitude & $\begin{array}{c}\text { Fe-pct. } \\
5\end{array}$ & $\begin{array}{c}\text { Mg-pct. } \\
s\end{array}$ & $\begin{array}{c}\text { Ca-pct. } \\
s\end{array}$ & $\begin{array}{c}\text { Ti-pct. } \\
5\end{array}$ & $\begin{array}{c}\text { Mn-pp而 } \\
5\end{array}$ & $\begin{array}{c}A q-p p \bar{i} \\
s\end{array}$ & $\begin{array}{c}\text { A5-pp伍 } \\
5\end{array}$ \\
\hline 422625 & 6532 & 1492451 & 7.00 & 2.00 & 3.0 & 1.000 & 2,000 & $N$ & N \\
\hline 422626 & $65 \quad 244$ & 1492620 & 7.00 & 2.00 & 5.0 & 1.000 & 1,000 & $N$ & $N$ \\
\hline 422627 & 65046 & 1492612 & 7.00 & 2.00 & 5.0 & 1.000 & 2,000 & $N$ & $N$ \\
\hline 422628 & 65036 & 149232 & 7.00 & 1.50 & 3.0 & 1.000 & 2,000 & $N$ & $N$ \\
\hline 422629 & 65026 & 1492138 & 7.00 & 2.00 & 5.0 & .700 & 2,000 & $N$ & $N$ \\
\hline 422630 & $65 \quad 127$ & 1492048 & 7.00 & 1.50 & 2.0 & .700 & 1,000 & $<.5$ & N \\
\hline 422631 & 65122 & 1491718 & 5.00 & 1.50 & 2.0 & .700 & 700 & $N$ & N \\
\hline 422632 & $65 \quad 224$ & 1491641 & 5.00 & 1.50 & 2.0 & .700 & 500 & 6.5 & N \\
\hline 422633 & $65 \quad 314$ & 1491515 & 7.00 & 2.00 & 3.0 & 1.000 & 700 & $N$ & N \\
\hline 422634 & 6523 & $14915 \quad 17$ & 7.00 & 2.00 & 3.0 & .700 & 700 & N & $N$ \\
\hline 422635 & 6512 & 1491417 & 3.00 & 1.50 & 3.0 & .700 & 500 & $N$ & $N$ \\
\hline 422636 & 6514 & 1491015 & 5.00 & 2.00 & 3.0 & 1.000 & 700 & N & N \\
\hline 422637 & $65 \quad 028$ & $149 \quad 933$ & 7.00 & 2.00 & 5.0 & 31.000 & 700 & $N$ & $N$ \\
\hline 422638 & 65055 & $149 \quad 522$ & 7.60 & 2.00 & 3.0 & 1.000 & 700 & $N$ & N \\
\hline 422639 & $65 \quad 154$ & 149714 & 5.00 & 2.00 & 3.0 & 1.000 & 500 & $N$ & $N$ \\
\hline 422640 & $65 \quad 229$ & 14993 & 7.00 & 2.00 & 3.0 & 1.000 & 1,000 & $H$ & $N$ \\
\hline 422641 & 65315 & 1491041 & 10.00 & 2.00 & 5.0 & 1.000 & 700 & $N$ & $N$ \\
\hline 422642 & $\begin{array}{lll}65 & 4 & 8\end{array}$ & 1491135 & 7.00 & 2.00 & 5.0 & 1.000 & 700 & $N$ & N \\
\hline 422643 & 65355 & $149 \quad 738$ & 5.00 & 1.50 & 3.0 & .700 & 500 & $N$ & $N$ \\
\hline 422644 & $65 \quad 437$ & $149 \quad 547$ & 7.00 & 2.00 & 3.0 & .700 & 500 & $N$ & $N$ \\
\hline 422645 & $65 \quad 515$ & $149 \quad 829$ & 7.00 & 2.00 & 3.0 & 1.000 & 500 & $N$ & N \\
\hline 422647 & $65 \quad 535$ & 1491036 & 7.00 & 1.50 & 3.0 & 1.000 & 1,000 & $N$ & $N$ \\
\hline 422648 & $65 \div 33$ & 14987 & 7.00 & 2.00 & 3.0 & .700 & 1,000 & N & $N$ \\
\hline 422649 & $65 \quad 659$ & 149533 & 7.00 & 2.00 & 3.0 & .700 & 700 & N & $N$ \\
\hline 422650 & 6580 & $149 \quad 625$ & 7.00 & 2.00 & 1.5 & .700 & 1,000 & $N$ & N \\
\hline 422748 & 65853 & $149 \quad 8 \quad 2$ & 5.00 & 1.50 & 2.0 & .700 & 700 & $<.5$ & $N$ \\
\hline 422749 & 65950 & 149611 & 5.00 & 1.50 & 2.0 & .700 & 1,000 & $<.5$ & $N$ \\
\hline 422750 & 651029 & 14959 & 7.00 & 2.00 & 2.0 & 1.000 & 1,000 & $\langle .5$ & $N$ \\
\hline 422751 & 651031 & $149 \quad 918$ & 7.00 & 1.50 & 5.0 & 1.000 & 1,000 & $N$ & $N$ \\
\hline 422752 & 651112 & 149718 & 10.00 & 2.00 & 3.0 & 1.000 & 1,000 & $N$ & N \\
\hline 422753 & 652017 & 1491112 & 7.00 & 1.50 & 3.0 & .700 & 1,000 & $N$ & $N$ \\
\hline 422754 & 65208 & 1491829 & 10.00 & 1.50 & 2.0 & 1.000 & 1,500 & $N$ & H \\
\hline 422755 & 652121 & $149 \quad 1547$ & 10.00 & 2.00 & 3.0 & .700 & 1,500 & $N$ & N \\
\hline 422756 & 65220 & 1491010 & 7.00 & 2.00 & 5.0 & 1.000 & 1,000 & $N$ & $N$ \\
\hline 422757 & 652345 & $149 \quad 834$ & 10.00 & 2.00 & 3.0 & 1.000 & 1,500 & $N$ & $N$ \\
\hline 422750 & $6519 \quad 2$ & 1485658 & 7.00 & 2.00 & 3.0 & .700 & 1,500 & .5 & $N$ \\
\hline 422759 & 652344 & $149 \quad 015$ & 7.00 & 1.50 & 3.0 & 1.000 & 1,000 & .5 & N \\
\hline 422760 & 651650 & 1484435 & 7.00 & 1.50 & 2.0 & 1.000 & 1,000 & .5 & $N$ \\
\hline 422761 & 651436 & $148 \quad 4543$ & 10.00 & 3.00 & 3.0 & 1.000 & 3,000 & 3.0 & $N$ \\
\hline 422762 & 651136 & 149746 & 5.00 & 1.50 & 2.0 & .700 & 1,500 & $(.5$ & $N$ \\
\hline 422763 & 651132 & 149159 & 7.00 & 1.50 & 1.5 & 1.000 & 2,000 & .7 & $N$ \\
\hline 422764 & 651049 & $149 \quad 132$ & 7.00 & 1.50 & 3.0 & 1.000 & 1,000 & $N$ & H \\
\hline 422765 & $65 \quad 924$ & 149151 & 5.00 & 1.50 & 3.0 & .700 & 2,000 & N & $N$ \\
\hline 422766 & $65 \quad 754$ & 14916 & 7.00 & 1.50 & 3.0 & 1.000 & 1,500 & $N$ & $N$ \\
\hline 422767 & $65 \quad 656$ & 149311 & 7.00 & 2.00 & 3.0 & 1.000 & 2,000 & N & H \\
\hline
\end{tabular}


TABLE 2.--RESULTS OF SPECTROGRAPHIC ANALYSES OF NURE STREAM-SEDIMENT SAMPLES FROM THE LIVENGOOD

AND HESTERN $1 / 3$ OF THE CIRCLE QUADRANGLES, ALASKA-Continued

\begin{tabular}{|c|c|c|c|c|c|c|c|c|c|c|}
\hline Sa㽢le & $\begin{array}{c}\text { B-ppo } \\
5\end{array}$ & $\begin{array}{c}\text { Ba-ppm } \\
5\end{array}$ & $\begin{array}{c}\text { Be-ppi } \\
5\end{array}$ & $\begin{array}{c}B i-p p \text { i } \\
5\end{array}$ & $\begin{array}{c}\text { Co-pp } \\
5\end{array}$ & $\begin{array}{c}C r-p p \\
5\end{array}$ & $\begin{array}{c}\text { Cu-ppi } \\
5\end{array}$ & $\begin{array}{l}\text { La-ppi } \\
5\end{array}$ & $\begin{array}{c}\text { Mo-ppi } \\
5\end{array}$ & $\begin{array}{c}\text { Nb-pp } \\
5\end{array}$ \\
\hline 422625 & 100 & 1,500 & 1.0 & $N$ & 15 & 100 & 50 & 100 & N & 20 \\
\hline 422626 & 70 & 1,000 & 2.0 & $N$ & 10 & 70 & 15 & 70 & $N$ & 30 \\
\hline 422627 & 70 & 700 & 1.5 & $N$ & 15 & 100 & 15 & 100 & $N$ & 20 \\
\hline 422628 & 150 & 1,500 & $\langle 1.0$ & $N$ & 15 & 200 & 50 & 50 & $N$ & $N$ \\
\hline 422629 & 100 & 1,500 & 11.0 & $N$ & 20 & 150 & 50 & 50 & N & $<20$ \\
\hline 422630 & 100 & 1,500 & 1.0 & $N$ & 20 & 100 & 30 & 30 & $N$ & $\$ 20$ \\
\hline 422631 & 70 & 1,500 & 1.0 & $N$ & 20 & 70 & 50 & 30 & N & $N$ \\
\hline 422632 & 50 & 1,500 & 1.0 & $N$ & 15 & 100 & 70 & 30 & $N$ & $N$ \\
\hline 422633 & 50 & 1,500 & $\langle 1.0$ & $N$ & 15 & 200 & 70 & 30 & $N$ & $N$ \\
\hline 422634 & 50 & 1,000 & 1.0 & $N$ & 20 & 100 & 70 & N & $\mathrm{N}$ & $N$ \\
\hline 422635 & 30 & 1,000 & 1.0 & $N$ & 10 & 70 & 30 & N & $N$ & $N$ \\
\hline 422636 & 70 & 1,500 & $\langle 1.0$ & $\mathrm{N}$ & 30 & 150 & 30 & 50 & $N$ & $<20$ \\
\hline 422637 & 70 & 1,500 & $\langle 1.0$ & $N$ & 20 & 200 & 70 & 50 & $N$ & $<20$ \\
\hline 422630 & 50 & 1,500 & $<1.0$ & $N$ & 20 & 150 & 70 & 50 & $\langle 5$ & $N$ \\
\hline 422639 & 50 & 1,000 & 1.0 & $N$ & 15 & 100 & 50 & 50 & $N$ & $N$ \\
\hline 422640 & 70 & 1,000 & $\langle 1.0$ & $N$ & 15 & 150 & 30 & 50 & $N$ & $<20$ \\
\hline 422641 & 70 & 2,000 & 1.0 & $N$ & 30 & 200 & 70 & 70 & $N$ & $<20$ \\
\hline 422642 & 50 & 2,000 & 1.0 & $N$ & 20 & 150 & 50 & 50 & $N$ & $<20$ \\
\hline 422643 & 70 & 1,000 & 11.0 & $\ddot{N}$ & 15 & 100 & 15 & 50 & N & 120 \\
\hline 422644 & 70 & 1,000 & 1.0 & $N$ & 20 & 150 & 30 & 70 & $N$ & $N$ \\
\hline 422645 & 70 & 1,000 & $\langle 1.0$ & $N$ & 20 & 150 & 20 & 70 & $N$ & $<20$ \\
\hline 422647 & 100 & 1,000 & 1.0 & $N$ & 15 & 200 & 20 & 70 & $N$ & $<20$ \\
\hline 422648 & 100 & 1,500 & 1.0 & $N$ & 15 & 200 & 30 & 100 & $N$ & $<20$ \\
\hline 422649 & 70 & 1,500 & 1.0 & $N$ & 15 & 150 & 30 & 30 & $N$ & $N$ \\
\hline 422650 & 100 & 1,500 & 1.0 & $N$ & 20 & 200 & 20 & 70 & $N$ & $N$ \\
\hline 422748 & 50 & 1,000 & 1.0 & $N$ & 15 & 100 & 20 & $N$ & $<5$ & $N$ \\
\hline 422749 & 70 & 1,000 & 1.0 & $N$ & 15 & 100 & 30 & $N$ & N & $N$ \\
\hline 422750 & 100 & 1,500 & 1.0 & $N$ & 20 & 150 & 30 & 30 & $N$ & $<20$ \\
\hline 422751 & 70 & 1,000 & $\langle 1.0$ & $N$ & 15 & 150 & 15 & 50 & $N$ & $N$ \\
\hline 422752 & 70 & 1,000 & 1.0 & $N$ & 20 & 150 & 50 & 50 & $N$ & $<20$ \\
\hline 422753 & 100 & 1,000 & $<1.0$ & $N$ & 15 & 150 & 15 & 50 & $N$ & $N$ \\
\hline 422754 & 100 & 700 & 1.0 & $N$ & 20 & 100 & 30 & 50 & $N$ & $<20$ \\
\hline 422755 & 150 & 1,500 & 1.0 & $N$ & 20 & 300 & 30 & 150 & $N$ & 20 \\
\hline 422756 & 100 & 2,000 & $\$ 1.0$ & $N$ & 20 & 150 & 50 & 50 & $N$ & $<20$ \\
\hline 422757 & 70 & 1,500 & $\$ 1.0$ & $N$ & 15 & 200 & 30 & 50 & $N$ & $<20$ \\
\hline 422758 & 70 & 1,500 & 1.0 & $N$ & 15 & 300 & 70 & $N$ & 5 & $N$ \\
\hline 422759 & 100 & 1,500 & 1.0 & $N$ & 15 & 200 & 30 & 50 & $N$ & $N$ \\
\hline 422760 & 70 & 1,500 & 1.0 & $N$ & 15 & 100 & 20 & 50 & $N$ & $N$ \\
\hline 422761 & 150 & 3,000 & 1.0 & $N$ & 20 & 200 & 70 & 70 & N & $<20$ \\
\hline 422762 & 100 & 2,000 & 1.0 & $N$ & 15 & 150 & 30 & 50 & $N$ & $<20$ \\
\hline 422763 & 150 & 1,500 & 1.0 & $N$ & 50 & 150 & 70 & 50 & $<5$ & $<20$ \\
\hline 422764 & 100 & 1,500 & 1.0 & $\mathrm{~N}$ & 15 & 150 & 30 & 50 & $\mathrm{~N}$ & $<20$ \\
\hline 422765 & 100 & 1,500 & 1.0 & $N$ & 10 & 70 & 30 & 30 & $\mathrm{~N}$ & N \\
\hline 422766 & 150 & 1,500 & 1.0 & $\mathrm{~N}$ & 15 & 150 & 30 & 50 & $\mathrm{~N}$ & 120 \\
\hline 422767 & 70 & 1,500 & 1.0 & $N$ & 20 & 200 & 50 & 50 & $N$ & $\mathrm{~N}$ \\
\hline
\end{tabular}


TABLE 2. - RESULTS OF SPECTRDGRAPHIC ANALYSES OF NURE STREAM-SEDIMENT SAMPLES FROM THE LIVENGDOD

AND HESTERN $1 / 3$ OF THE CIRCLE QUADRANGLES, ALASKA--ContinUEd

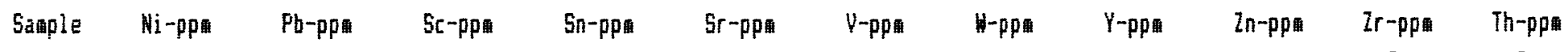

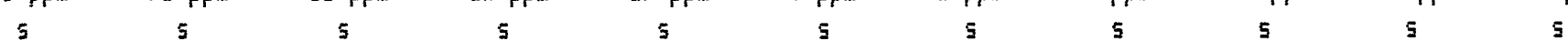

\begin{tabular}{|c|c|c|c|c|c|c|c|c|c|c|}
\hline 422625 & 30 & 70 & 20 & $N$ & 500 & 150 & $N$ & 50 & $N$ & 700 \\
\hline $422 b 2 b$ & 20 & 20 & 20 & 10 & 500 & 150 & $N$ & 50 & N & 1,000 \\
\hline 422627 & 20 & 20 & 20 & $<10$ & 500 & 150 & $N$ & 50 & $N$ & 1,000 \\
\hline 422628 & 70 & 15 & 20 & N & 300 & 200 & N & 50 & N & 500 \\
\hline 422629 & 50 & 20 & 20 & $N$ & 300 & 150 & N & 50 & $N$ & 200 \\
\hline 422630 & 50 & 20 & 20 & $N$ & 200 & 200 & N & 50 & N & 300 \\
\hline 422631 & 70 & 20 & 20 & $H$ & 200 & 150 & H & 30 & $N$ & 150 \\
\hline $422 b 32$ & 70 & 20 & 20 & $N$ & 150 & 200 & $N$ & 50 & N & 150 \\
\hline 422633 & 50 & 20 & 20 & $N$ & 300 & 200 & $N$ & 50 & $N$ & 300 \\
\hline 422634 & 70 & 15 & 15 & N & 150 & 150 & $N$ & 30 & 200 & 150 \\
\hline 422635 & 30 & 10 & 15 & $N$ & 150 & 100 & N & 20 & $N$ & 150 \\
\hline 422636 & 50 & 30 & 30 & N & 200 & 200 & N & 50 & N & 200 \\
\hline 422637 & 50 & 30 & 30 & $N$ & 300 & 200 & N & 50 & $N$ & 500 \\
\hline 422638 & 50 & 30 & 30 & N & 300 & 200 & N & 50 & N & 200 \\
\hline 422639 & 30 & 20 & 20 & $N$ & 300 & 150 & $N$ & 30 & $N$ & 300 \\
\hline 422640 & 30 & 20 & 20 & N & 300 & 150 & N & 150 & N & 500 \\
\hline 422641 & 50 & 30 & 30 & $N$ & 300 & 300 & $N$ & 50 & N & 300 \\
\hline 422642 & 50 & 20 & 30 & H & 300 & 200 & $H$ & 30 & $N$ & 300 \\
\hline 422643 & 30 & 20 & 20 & $N$ & 300 & 150 & N & 30 & N & 500 \\
\hline 422644 & 50 & 20 & 20 & N & 300 & 150 & $N$ & 50 & N & 300 \\
\hline 422645 & 30 & 20 & 15 & $N$ & 300 & 150 & $N$ & 50 & $N$ & 300 \\
\hline 422647 & 50 & 20 & 20 & $\mathrm{H}$ & 300 & 150 & $N$ & 50 & $N$ & 500 \\
\hline 422648 & 70 & 20 & 30 & $N$ & 200 & 200 & $N$ & 50 & $N$ & 500 \\
\hline $422 b 49$ & 50 & 20 & 20 & N & 300 & 150 & $N$ & 50 & $N$ & 700 \\
\hline 422650 & 70 & 15 & 20 & $N$ & 200 & 150 & N & 30 & $N$ & 300 \\
\hline 422748 & 50 & 15 & 20 & $H$ & 100 & 150 & $N$ & 30 & $N$ & 150 \\
\hline 422749 & 50 & 15 & 15 & $N$ & 100 & 150 & H & 30 & N & 300 \\
\hline 422750 & 50 & 20 & 20 & H & 200 & 200 & $N$ & 30 & $H$ & 300 \\
\hline 422751 & 20 & 20 & 20 & $N$ & 300 & 150 & $N$ & 30 & N & 300 \\
\hline 422752 & 50 & 30 & 20 & N & 200 & 200 & $N$ & 50 & N & 500 \\
\hline 422753 & 30 & 20 & 20 & N & 200 & 150 & $N$ & 30 & N & 700 \\
\hline 422754 & 30 & 30 & 20 & N & 200 & 150 & $H$ & 30 & N & 700 \\
\hline 422755 & 70 & 15 & 20 & H & 200 & 300 & N & 70 & N & 1,000 \\
\hline $42275 b$ & 50 & 20 & 30 & $N$ & 200 & 200 & $H$ & 50 & N & 500 \\
\hline 422757 & 50 & 15 & 20 & $N$ & 200 & 300 & N & 50 & $N$ & 700 \\
\hline 422758 & 100 & 15 & 20 & $N$ & 150 & 200 & $N$ & 50 & N & 3 \\
\hline 422759 & 70 & 20 & 20 & $N$ & 200 & 200 & N & 50 & $N$ & 50 \\
\hline 422760 & 30 & 15 & 20 & H & 200 & 200 & $N$ & 50 & N & 50 \\
\hline 422761 & 70 & 70 & 20 & $N$ & 300 & 200 & $<50$ & 70 & 200 & 30 \\
\hline 422762 & 50 & 50 & 20 & $N$ & 300 & 200 & $N$ & 50 & 200 & 300 \\
\hline 422763 & 100 & 70 & 30 & $N$ & 200 & 200 & N & 50 & 200 & 30 \\
\hline 422764 & 30 & 30 & 20 & H & 300 & 200 & N & 50 & $N$ & 3 \\
\hline 422765 & 30 & 20 & 15 & $N$ & 200 & 200 & $N$ & 30 & $N$ & 20 \\
\hline 422766 & 30 & 30 & 20 & $N$ & 300 & 200 & $N$ & 50 & N & 70 \\
\hline 422767 & 70 & 20 & 20 & $N$ & 200 & 200 & $N$ & 30 & $N$ & 200 \\
\hline
\end{tabular}


TABLE 2.--RESULTS OF SPECTRDGRAPHIC ANALYSES OF NURE STREAM-SEDIMENT SAMPLES FROM THE LIVENGODD AND WESTERN $1 / 3$ OF THE CIRCLE QUADRANGLES, ALASKA--Continued

\begin{tabular}{|c|c|c|c|c|c|c|c|c|c|}
\hline Sample & Latitude & Longitude & $\begin{array}{c}\text { Fe-pct. } \\
5\end{array}$ & $\begin{array}{c}\text { Mg-pct. } \\
5\end{array}$ & $\begin{array}{c}\text { Ca-pct. } \\
5\end{array}$ & $\begin{array}{c}\text { Ti-pct. } \\
5\end{array}$ & $\begin{array}{c}\text { Mn-pp } \\
s\end{array}$ & $\begin{array}{c}A q-p p \mathbb{A} \\
5\end{array}$ & $\begin{array}{c}\text { As-ppm } \\
5\end{array}$ \\
\hline 422768 & $65 \quad 522$ & 149051 & 7.00 & 3.00 & 5.0 & 1.000 & 3,000 & N & $N$ \\
\hline 422769 & 65311 & $149 \quad 011$ & 7.00 & 3.00 & 5.0 & 1.000 & 1,500 & N & $N$ \\
\hline 422770 & 65153 & 14910 & 10.00 & 3.00 & 5.0 & $\$ 1.000$ & 2,000 & N & $N$ \\
\hline 422771 & 65046 & 1485945 & 7.00 & 2.00 & 7.0 & 1.000 & 1,500 & N & $N$ \\
\hline 422772 & $65 \quad 020$ & $14854 \quad 12$ & 7.00 & 2.00 & 5.0 & 1.000 & 1,000 & N & $H$ \\
\hline 422773 & 6533 & $148 \quad 6 \quad 38$ & 7.00 & 1.50 & 3.0 & 1.000 & 500 & $N$ & $N$ \\
\hline 422774 & 65239 & 1481125 & 10.00 & 2.00 & 7.0 & .700 & 1,000 & N & $N$ \\
\hline 422775 & 654851 & 1491131 & .15 & .20 & 3.0 & .030 & 200 & N & $N$ \\
\hline 422776 & 654651 & 149149 & 10.00 & 2.00 & 3.0 & 1.000 & 1,000 & N & $N$ \\
\hline 422777 & 654654 & $14938 \quad 9$ & 7.00 & 2.00 & 7.0 & 1.000 & 1,000 & $N$ & $N$ \\
\hline 422778 & $6533 \quad 2$ & 1494520 & .30 & .20 & .1 & .200 & 200 & 1.5 & $N$ \\
\hline 422779 & 653023 & 149457 & .15 & .05 & .1 & .020 & 50 & $N$ & $N$ \\
\hline 435600 & 654951 & 1463957 & 7.00 & 1.50 & 1.0 & 1.000 & 1,000 & N & $N$ \\
\hline 435601 & 654919 & 1463329 & 10.00 & 1.50 & 3.0 & .700 & 2,000 & .5 & $N$ \\
\hline 435602 & 654936 & 1463449 & 7.00 & 1.50 & 3.0 & 1.000 & 3,000 & .5 & $N$ \\
\hline 435603 & 655256 & 1462736 & 15.00 & .50 & 3.0 & .300 & 75,000 & N & $N$ \\
\hline 435604 & 655232 & $146 \quad 26 \quad 17$ & 10.00 & 2.00 & 3.0 & 1.000 & 2,000 & H & $N$ \\
\hline 435605 & 655242 & 1462152 & 7.00 & 1.50 & 3.0 & .700 & 1,500 & $N$ & $N$ \\
\hline 435606 & 655120 & $146 \quad 1730$ & 7.00 & 1.50 & 3.0 & 1.000 & 1,000 & N & $N$ \\
\hline 435608 & 65503 & $14618 \quad 28$ & 10.00 & 1.50 & 3.0 & 1.000 & 1,000 & $<.5$ & $N$ \\
\hline 435610 & 655334 & $146 \quad 1454$ & 5.00 & 1.50 & 3.0 & 1.000 & 1,000 & $N$ & $N$ \\
\hline 435611 & 655348 & $146 \quad 13 \quad 14$ & 5.00 & 1.50 & 5.0 & 1.000 & 1,000 & N & $N$ \\
\hline 435612 & $6556 \quad 8$ & $146 \quad 1245$ & 7.00 & 1.50 & 3.0 & 1.000 & 700 & $N$ & $N$ \\
\hline 435614 & 655857 & 1462111 & 10.00 & 1.50 & 2.0 & 1.000 & 1,000 & $N$ & $N$ \\
\hline 435616 & 65567 & 146256 & 1.50 & .30 & 3.0 & .150 & 3,000 & $N$ & $N$ \\
\hline 435618 & 655855 & $146 \quad 24 \quad 37$ & 7.00 & 1.50 & 3.0 & 1.000 & 1,000 & $N$ & $N$ \\
\hline 435619 & 655829 & 1462429 & 10.00 & 2.00 & 3.0 & $>1.000$ & 2,000 & $N$ & $N$ \\
\hline 435621 & 655735 & 146316 & 1.00 & .30 & 2.0 & .200 & 700 & N & $N$ \\
\hline 435622 & 65567 & 146308 & 7.00 & 2.00 & 5.0 & 1.000 & 700 & N & $\mathrm{N}$ \\
\hline 435628 & $6556 \quad b$ & 1464439 & .50 & .20 & 5.0 & .050 & 700 & $N$ & $N$ \\
\hline 435630 & $6558 \quad 45$ & 146589 & 10.00 & 2.00 & 5.0 & 31.000 & 2,000 & N & $N$ \\
\hline 435631 & 655535 & 1465649 & 10.00 & 2.00 & 5.0 & 1.000 & 3,000 & $N$ & $N$ \\
\hline 435632 & 654521 & 1463329 & 10.00 & 1.50 & 3.0 & 1.000 & 2,000 & 6.5 & $N$ \\
\hline 435633 & 654442 & 1463243 & 10.00 & 1.50 & 2.0 & 1.000 & 2,000 & .5 & $N$ \\
\hline 435634 & 654317 & 1463353 & 10.00 & 2.00 & 3.0 & 1.000 & 2,000 & $<.5$ & $N$ \\
\hline 435635 & 654252 & 1463130 & 7.00 & 1.50 & 3.0 & 1.000 & 1,000 & .5 & N \\
\hline 435636 & $6524 \quad 47$ & $146 \quad 6 \quad 32$ & 1.00 & .30 & 1.0 & .150 & 150 & .5 & N \\
\hline 435637 & 652520 & 146918 & 2.00 & .50 & .2 & .150 & 150 & $<.5$ & N \\
\hline 435678 & 652730 & 146952 & 5.00 & .20 & .7 & .100 & 200 & 6.5 & N \\
\hline 435639 & 652833 & 146933 & 5.00 & 1.00 & 1.0 & .200 & 500 & $<.5$ & N \\
\hline 435640 & 652854 & 146946 & 7.00 & 1.50 & .5 & .300 & 500 & .5 & N \\
\hline 435641 & $6529 \quad 12$ & 1461645 & 5.00 & 1.50 & .7 & 1.000 & 1,000 & $N$ & N \\
\hline 435642 & 65298 & $146 \quad 4255$ & 7.00 & 1.50 & .3 & 1.000 & 1,000 & N & N \\
\hline 435643 & 653115 & 1461120 & 7.00 & 1.50 & .5 & .700 & 1,000 & N & N \\
\hline 435644 & 653051 & 1461059 & 7.00 & 1.50 & 2.0 & 1.000 & 1,000 & $N$ & N \\
\hline
\end{tabular}


TABLE 2. --RESULTS OF SPECTROGRAPHIC ANALYSES OF NURE STREAM-SEDIMENT SAMPLES FROM THE LIVENGDDD

AND WESTERN $1 / 3$ OF THE CIRCLE QUADRAMGLES, ALASKA--Continued

\begin{tabular}{|c|c|c|c|c|c|c|c|c|c|c|}
\hline Sample & $\begin{array}{c}\text { B-ppm } \\
5\end{array}$ & $\begin{array}{l}\text { Ba-ppi } \\
5\end{array}$ & $\begin{array}{c}\text { Ee-pp } \\
5\end{array}$ & $\begin{array}{c}\text { Ei-pp畐 } \\
5\end{array}$ & $\begin{array}{c}\text { Co-ppi } \\
5\end{array}$ & $\begin{array}{c}\text { Cr-ppm } \\
5\end{array}$ & $\begin{array}{c}\text { Cu-pp } \\
5\end{array}$ & $\begin{array}{l}\text { La-ppi } \\
\quad \mathrm{i}\end{array}$ & $\begin{array}{c}\text { Mo-pp } \\
5\end{array}$ & 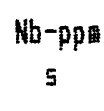 \\
\hline 422768 & 100 & 2,000 & 1.0 & $N$ & 30 & 200 & 100 & 50 & $N$ & $<20$ \\
\hline 422769 & 100 & 1,500 & $\langle 1.0$ & $N$ & 20 & 300 & 50 & 20 & $N$ & 20 \\
\hline 422770 & 50 & 2,000 & $\langle 1.0$ & $N$ & 30 & 300 & 70 & $N$ & $N$ & $N$ \\
\hline 422771 & 70 & 2,000 & 1.0 & $N$ & 15 & 150 & 50 & 50 & $N$ & $N$ \\
\hline 422772 & 70 & 1,500 & 1.0 & $N$ & 15 & 150 & 20 & 50 & $N$ & $<20$ \\
\hline 422773 & 50 & 1,500 & 1.0 & $N$ & 15 & 100 & 100 & $H$ & $<5$ & $N$ \\
\hline 422774 & 100 & 2,000 & 1.0 & $N$ & 15 & 100 & 30 & 50 & $N$ & $<20$ \\
\hline 422775 & $<10$ & 150 & 41.0 & $N$ & $N$ & $H$ & 5 & $N$ & $N$ & $N$ \\
\hline 422776 & 100 & 1,500 & 1.0 & $N$ & 20 & 150 & 50 & 50 & $N$ & $<20$ \\
\hline 422777 & 70 & 1,500 & 1.0 & $N$ & 15 & 100 & 20 & 50 & $N$ & 20 \\
\hline 422778 & $\langle 10$ & 500 & 1.0 & $N$ & $N$ & 20 & 10 & $N$ & $N$ & $N$ \\
\hline 422779 & $N$ & 150 & 1.0 & $N$ & $N$ & $N$ & $\langle 5$ & $N$ & $N$ & $N$ \\
\hline 435600 & 150 & 1,000 & 1.5 & $N$ & 20 & 100 & 30 & 70 & $<5$ & $<20$ \\
\hline 435601 & 150 & 3,000 & 1.0 & $N$ & 20 & 150 & 50 & 70 & 5 & $<20$ \\
\hline 435602 & 100 & 2,000 & 1.5 & $N$ & 70 & 150 & 50 & 70 & $N$ & $<20$ \\
\hline 435603 & 10 & 1,500 & 1.5 & $N$ & 50 & 20 & 20 & $N$ & $<5$ & $N$ \\
\hline 435604 & 100 & 2,000 & 1.0 & $N$ & 20 & 200 & 50 & 70 & $N$ & $<20$ \\
\hline 435605 & 100 & 2,000 & 1.0 & $N$ & 20 & 200 & 50 & 50 & 7 & $<20$ \\
\hline 435606 & 100 & 1,500 & 1.0 & $N$ & 20 & 150 & 50 & 50 & $<5$ & $\langle 20$ \\
\hline 435608 & 100 & 2,000 & 1.0 & $N$ & 20 & 200 & 50 & 70 & 45 & 120 \\
\hline 435610 & 100 & 2,000 & 1.0 & $N$ & 15 & 100 & 50 & 30 & $N$ & $N$ \\
\hline 435611 & 70 & 2,000 & 1.0 & $N$ & 15 & 150 & 30 & 50 & $N$ & $\langle 20$ \\
\hline 435612 & 70 & 2,000 & 1.0 & $N$ & 20 & 150 & 50 & 50 & $N$ & $<20$ \\
\hline 435614 & 100 & 2,000 & 1.5 & $N$ & 20 & 200 & 50 & 50 & 5 & 120 \\
\hline 435616 & $\langle 10$ & 500 & 1.5 & $N$ & 65 & $<10$ & 20 & $N$ & $N$ & $N$ \\
\hline 435618 & 100 & 2,000 & 1.0 & $N$ & 15 & 150 & 30 & 70 & N & $<20$ \\
\hline 435619 & 70 & 700 & $\langle 1.0$ & $N$ & 15 & 200 & 70 & 30 & $N$ & $N$ \\
\hline 435621 & 20 & 500 & 1.0 & $N$ & $N$ & 20 & 10 & $N$ & $N$ & $N$ \\
\hline 435622 & 100 & 2,000 & 1.0 & $N$ & 15 & 200 & 30 & 50 & N & $N$ \\
\hline 435628 & 10 & 300 & 1.0 & $N$ & $N$ & 10 & 10 & $N$ & $N$ & $N$ \\
\hline 435630 & 100 & 2,000 & 1.0 & $N$ & 30 & 200 & 70 & 70 & $N$ & $<20$ \\
\hline 435631 & 150 & 2,000 & 1.0 & N & 20 & 200 & 70 & 50 & $N$ & $N$ \\
\hline 435632 & 100 & 3,000 & 1.0 & $N$ & 20 & 70 & 50 & 50 & $N$ & $N$ \\
\hline 435633 & 200 & 5,000 & 1.5 & $N$ & 20 & 150 & 70 & 70 & 7 & $<20$ \\
\hline 435634 & 100 & 2,000 & 1.0 & $N$ & 20 & 100 & 30 & 70 & $<5$ & $<20$ \\
\hline 435635 & 150 & 3,000 & 1.0 & $N$ & 15 & 200 & 50 & 50 & 5 & $<20$ \\
\hline 435636 & 410 & 300 & 1.5 & $<10$ & $N$ & 20 & 15 & 20 & $N$ & $N$ \\
\hline 435637 & $<10$ & 700 & 1.5 & $N$ & 7 & 30 & 30 & 20 & $N$ & $N$ \\
\hline 435638 & $N$ & 300 & 1.5 & $N$ & N & $\langle 10$ & 15 & $N$ & $N$ & $N$ \\
\hline 435639 & 50 & 700 & 1.5 & $N$ & 7 & 50 & 50 & 200 & $N$ & $N$ \\
\hline 435640 & 100 & 1,500 & 1.5 & $N$ & 15 & 100 & 50 & 150 & $N$ & $N$ \\
\hline 435641 & 200 & 1,000 & 2.0 & $N$ & 15 & 100 & 15 & 70 & $<5$ & $<20$ \\
\hline 435642 & 200 & 1,000 & 2.0 & $N$ & 15 & 100 & 15 & 50 & 65 & 120 \\
\hline 435643 & 150 & 1,500 & 2.0 & $N$ & 15 & 150 & 20 & 70 & $<5$ & $\measuredangle 20$ \\
\hline 435644 & 100 & 1,500 & 2.0 & $N$ & 15 & 200 & 30 & 70 & $<5$ & $<20$ \\
\hline
\end{tabular}


TABLE 2. - RESUL TS OF SPECTROGRAPHIC ANALYSES OF NURE STREAM-SEDIMENT SAMPLES FFOM THE LIVEMgDOD

AND WESTERN $1 / 3$ OF THE CIRCLE QUADRANGLES, ALASKA--ContinUEd

\begin{tabular}{|c|c|c|c|c|c|c|c|c|c|c|c|}
\hline Sample & $\begin{array}{c}\text { Ni-ppi } \\
5\end{array}$ & $\begin{array}{c}\mathrm{Pb}-\mathrm{ppm} \text { it } \\
5\end{array}$ & $\begin{array}{c}\text { Sc-ppm } \\
5\end{array}$ & $\begin{array}{c}S_{n}-p p \text { m } \\
5\end{array}$ & $\begin{array}{c}\text { Sr-ppa } \\
s\end{array}$ & $\begin{array}{c}\text { V-ppm } \\
s\end{array}$ & $\underset{5}{W-p p \text { m }}$ & $\begin{array}{c}y-p p \text { M } \\
5\end{array}$ & $\begin{array}{c}\text { Zn-pp } \\
5\end{array}$ & $\begin{array}{c}2 r-p p \text { i } \\
5\end{array}$ & $\begin{array}{c}\text { Th-ppin } \\
5\end{array}$ \\
\hline 422768 & 70 & 20 & 30 & N & 300 & 300 & $N$ & 50 & 200 & 300 & $N$ \\
\hline 422769 & 70 & 20 & 30 & $N$ & 200 & 300 & $N$ & 30 & $N$ & 300 & $N$ \\
\hline 422770 & 70 & 20 & 30 & $N$ & 200 & 300 & $N$ & 30 & $<200$ & 200 & $N$ \\
\hline 422771 & 50 & 20 & 20 & $N$ & 300 & 200 & $N$ & 50 & $N$ & 200 & $N$ \\
\hline 422772 & 30 & 15 & 20 & $N$ & 300 & 200 & $N$ & 30 & $H$ & 300 & $N$ \\
\hline 422773 & 50 & 15 & 50 & $N$ & 200 & 200 & $N$ & 50 & $N$ & 150 & $N$ \\
\hline 422774 & 30 & 20 & 20 & $N$ & 300 & 150 & $N$ & 50 & $N$ & 500 & $N$ \\
\hline 422775 & N & $H$ & N & $N$ & $N$ & 15 & $N$ & 10 & $N$ & 10 & $N$ \\
\hline $42277 b$ & 50 & 20 & 20 & $N$ & 300 & 200 & $N$ & 50 & $N$ & 500 & $\mathrm{~N}$ \\
\hline 422777 & 30 & 15 & 20 & $N$ & 300 & 150 & $N$ & 50 & $N$ & 700 & $N$ \\
\hline 422778 & 5 & 70 & 5 & $N$ & $<100$ & 70 & $N$ & 15 & $N$ & 100 & N \\
\hline 422779 & $N$ & $N$ & $N$ & $N$ & $N$ & 15 & $N$ & 10 & $N$ & 10 & $N$ \\
\hline 435600 & 50 & 20 & 20 & $N$ & 200 & 100 & $N$ & 50 & H & 500 & $N$ \\
\hline 435601 & 70 & 70 & 20 & $N$ & 300 & 200 & $N$ & 50 & 200 & 300 & $N$ \\
\hline 435602 & 70 & 30 & 20 & $N$ & 300 & 200 & $N$ & 70 & $N$ & 300 & $N$ \\
\hline 435600 & 30 & 15 & 7 & $N$ & 100 & 100 & $N$ & 20 & 200 & 70 & $N$ \\
\hline 435604 & 50 & 30 & 20 & $N$ & 300 & 150 & $N$ & 70 & $H$ & 500 & $N$ \\
\hline 435605 & 70 & 100 & 20 & 30 & 300 & 150 & $N$ & 50 & $N$ & 300 & $N$ \\
\hline 435606 & 50 & 20 & 15 & $N$ & 300 & 200 & $N$ & 50 & $N$ & 500 & $N$ \\
\hline 435608 & 50 & 20 & 20 & $N$ & 300 & 200 & $N$ & 70 & $N$ & 70 & $N$ \\
\hline 435610 & 30 & 20 & 15 & $H$ & 300 & 150 & $N$ & 30 & $N$ & 200 & $N$ \\
\hline 435611 & 30 & 30 & 20 & $N$ & 500 & 200 & $N$ & 50 & $N$ & 500 & $N$ \\
\hline 435612 & 50 & 20 & 20 & $N$ & 300 & 200 & $N$ & 50 & $N$ & 300 & H \\
\hline 435614 & 70 & 1,000 & 20 & 10 & 200 & 200 & $N$ & 70 & 500 & 700 & $N$ \\
\hline $43561 b$ & 20 & 10 & 5 & $N$ & $N$ & 70 & $N$ & 15 & $N$ & 50 & $N$ \\
\hline 435618 & 50 & 20 & 15 & $N$ & 300 & 200 & $N$ & 50 & $N$ & 500 & $H$ \\
\hline 435619 & 50 & 20 & 20 & $N$ & 300 & 150 & $N$ & 30 & $N$ & 300 & H \\
\hline 435621 & 10 & 20 & 5 & $N$ & $N$ & 50 & $N$ & 15 & $N$ & 100 & N \\
\hline 435622 & 30 & 50 & 20 & $N$ & 500 & 200 & $N$ & 50 & $N$ & 500 & N \\
\hline 435628 & $N$ & 10 & $N$ & $N$ & $N$ & 30 & $\mathrm{~N}$ & 10 & $N$ & 30 & N \\
\hline 435630 & 70 & 50 & 30 & $N$ & 200 & 300 & $N$ & 50 & $N$ & 500 & N \\
\hline 435631 & 30 & 30 & 20 & $N$ & 300 & 500 & $N$ & 70 & $N$ & 1,000 & N \\
\hline 435632 & 50 & 50 & 20 & $N$ & 300 & 300 & $N$ & 50 & $N$ & 500 & N \\
\hline 435633 & 100 & 50 & 20 & $N$ & 200 & 300 & $N$ & 50 & $N$ & 300 & H \\
\hline 435634 & 50 & 50 & 20 & $H$ & 300 & 200 & $N$ & 50 & $N$ & 500 & N \\
\hline 435635 & 50 & 50 & 30 & $N$ & 200 & 500 & $N$ & 50 & $N$ & 300 & H \\
\hline 435636 & 10 & 70 & 5 & $N$ & $N$ & 70 & $N$ & 15 & $N$ & 30 & 100 \\
\hline 435637 & 50 & 20 & 7 & $N$ & $N$ & 70 & $N$ & 15 & $N$ & 70 & $N$ \\
\hline 435638 & 20 & 10 & 7 & $N$ & N & 50 & $N$ & 30 & $N$ & 50 & $N$ \\
\hline 435637 & 30 & 30 & 20 & $N$ & $\measuredangle 100$ & 100 & $N$ & 70 & $N$ & 70 & $N$ \\
\hline 435640 & 70 & 70 & 20 & $N$ & 150 & 150 & $N$ & 50 & $H$ & 150 & $N$ \\
\hline 435641 & 30 & 30 & 15 & 20 & 150 & 70 & $N$ & 70 & $\mathrm{~N}$ & 500 & $<100$ \\
\hline 435642 & 30 & 50 & 15 & 15 & 150 & 70 & $N$ & 30 & $N$ & 300 & $N$ \\
\hline 435643 & 50 & 50 & 20 & 10 & 200 & 70 & $N$ & 30 & $N$ & 300 & N \\
\hline 435644 & 50 & 70 & 20 & 10 & 200 & 150 & $H$ & 50 & $<200$ & 300 & $N$ \\
\hline
\end{tabular}


TABLE 2.--RESULTS OF SPECTROGRAFHIC AMALYSES OF NURE STREAM-SEDIMENT SAMPLES FROM THE LIVENGOOD AND WESTERN $1 / 3$ OF THE CIRCLE QUADRANGLES, ALA5KA--ContinURd

\begin{tabular}{|c|c|c|c|c|c|c|c|c|c|}
\hline Satiple & Latitude & Long̣itude & $\begin{array}{c}\text { Fe-pct. } \\
s\end{array}$ & $\begin{array}{c}M_{q}-p c t . \\
s\end{array}$ & $\begin{array}{c}\text { Ca-pct. } \\
s\end{array}$ & $\begin{array}{c}\text { Ti-pct. } \\
s\end{array}$ & $\begin{array}{c}\text { Mn-ppi } \\
5\end{array}$ & $\begin{array}{c}A g-p p \text { 而 } \\
5\end{array}$ & $\begin{array}{c}\text { A5-pp } \\
5\end{array}$ \\
\hline 435645 & 653132 & $146 \quad 1324$ & 7.00 & 1.50 & 1.5 & 1.000 & 1,500 & $N$ & N \\
\hline 435646 & $6532 \quad 2$ & $146 \quad 1259$ & 10.00 & 1.50 & 1.0 & 1.000 & 1,500 & $N$ & $N$ \\
\hline 435647 & 653240 & $146 \quad 1354$ & 7.00 & 1.50 & 1.0 & 1.000 & 1,000 & $N$ & $N$ \\
\hline 435648 & 653342 & 146160 & 7.00 & 1.50 & 2.0 & 1.000 & 1,000 & $N$ & $N$ \\
\hline 435649 & 653333 & 146181 & 7.00 & 1.50 & .2 & .500 & 700 & N & $N$ \\
\hline 435650 & 653316 & $146 \quad 18 \quad 0$ & 10.00 & 1.50 & .7 & 1.000 & 1,500 & $N$ & $N$ \\
\hline 435651 & $6534 \quad 1$ & $14 b \quad 1932$ & 1.00 & .30 & 2.0 & .070 & 150 & N & $N$ \\
\hline 435652 & 653646 & 1462351 & 10.00 & 2.00 & 1.0 & 1.000 & 1,000 & $N$ & $N$ \\
\hline 435653 & $6537 \quad 14$ & 146220 & 10.00 & 2.00 & 2.0 & 1.000 & 1,500 & N & $N$ \\
\hline 435654 & 653724 & 1462251 & 10.00 & 2.00 & 2,0 & 1.000 & 1,500 & $N$ & $N$ \\
\hline 435655 & 653750 & $146 \quad 218$ & 10.00 & 1.50 & 2.0 & 1.000 & 1,000 & $<.5$ & $\mathrm{H}$ \\
\hline 435656 & 65396 & $146 \quad 2358$ & 5.00 & 1.50 & 2.0 & .700 & 1,000 & $N$ & $N$ \\
\hline 435557 & 653916 & $146 \quad 25 \quad 17$ & 7.00 & 2.00 & 2.0 & 1.000 & 1,500 & N & $N$ \\
\hline 435658 & 654111 & $146 \quad 24 \quad 18$ & 7.00 & 1.50 & 2.0 & 1.000 & 1,500 & $<.5$ & $N$ \\
\hline 435659 & 654055 & 1462339 & 5.00 & 1.50 & 1.5 & .700 & 1,000 & $N$ & $N$ \\
\hline 435660 & 654116 & $14622 \quad 27$ & 7.00 & 1.50 & 2.0 & 1.000 & 1,000 & $N$ & N \\
\hline 435661 & 654226 & 1462111 & 7.00 & 1.50 & 3.0 & .700 & 700 & N & $N$ \\
\hline 435662 & 65429 & $146 \quad 2035$ & 7.00 & 1.50 & 3.0 & 1.000 & 1,500 & $N$ & $N$ \\
\hline 435663 & 654341 & 1461618 & 7.00 & 1.50 & 3.0 & .700 & 1,000 & $N$ & $N$ \\
\hline 435664 & 654328 & 1461530 & 10.00 & 1.50 & .5 & 1.000 & 1,500 & $N$ & $N$ \\
\hline 435665 & 654457 & 1462036 & 15.00 & 1.00 & .2 & .700 & 150 & $N$ & $N$ \\
\hline 435666 & 654457 & 1462139 & 15.00 & 1.50 & 1.0 & 1.000 & 300 & $N$ & $N$ \\
\hline 4356667 & 654546 & $146 \quad 23 \quad 49$ & 7.00 & 1.50 & 1.5 & 1.000 & 1,000 & $<.5$ & $N$ \\
\hline 435668 & $65 \quad 4739$ & 1462537 & .20 & .10 & .5 & .030 & 150 & $N$ & $N$ \\
\hline 435669 & 654739 & $146 \quad 31 \quad 17$ & 7.00 & 2.00 & 3.0 & 1.000 & 1,000 & $N$ & $\mathrm{~N}$ \\
\hline 435670 & 655133 & 1462850 & 10.00 & 1.50 & 1.5 & 1.000 & 2,000 & 6.5 & N \\
\hline 435671 & 655130 & $146 \quad 27 \quad 42$ & 10.00 & 1.50 & 2.0 & 1.000 & 2,000 & $<.5$ & $N$ \\
\hline 435672 & 65528 & $146 \quad 2729$ & 7.00 & 2.00 & 3.0 & 1.000 & 1,000 & $N$ & $N$ \\
\hline 435673 & 654910 & 1463844 & .70 & .20 & 5.0 & .100 & 500 & N & $N$ \\
\hline 435674 & $6549 \quad 7$ & $14 b 4049$ & 10.00 & 2.00 & 2.0 & 1.000 & 2,000 & N & $N$ \\
\hline 435675 & 654953 & $146 \quad 4633$ & 7.00 & 2.00 & 3.0 & 1.000 & 1,500 & N & $N$ \\
\hline 435676 & 655020 & $146 \quad 45 \quad 47$ & 7.00 & 1.50 & 3.0 & 1.000 & 1,500 & $N$ & $H$ \\
\hline 435677 & 65509 & 1465743 & 5.00 & 1.50 & 2.0 & 1.000 & 500 & $<.5$ & $N$ \\
\hline 435678 & 655034 & $14657 \quad 2$ & 5.00 & 2.00 & 2.0 & 1.000 & 1,000 & $N$ & $N$ \\
\hline 435679 & $6549 \quad 18$ & 1465912 & 5.00 & 1.50 & 2.0 & .500 & 1,000 & $N$ & N \\
\hline 435680 & 654947 & 1465943 & 3.00 & 1.50 & 2.0 & .500 & 700 & $N$ & $N$ \\
\hline 435681 & 655115 & 1465136 & 5.00 & 1.50 & .7 & .500 & 1,000 & $N$ & N \\
\hline 435682 & 65517 & 1465226 & 5.00 & 1.50 & 1.5 & .700 & 1,000 & $N$ & N \\
\hline 43566.3 & 655031 & 1465134 & 5.00 & 1.50 & 2.0 & .500 & 1,000 & N & N \\
\hline 435684 & 654739 & 1464250 & 5.00 & 1.50 & 2.0 & .700 & 700 & $N$ & N \\
\hline 435685 & 654650 & $14638 \quad 21$ & 5.00 & 1.50 & 2.0 & .700 & 700 & $N$ & H \\
\hline 435686 & 654543 & 146414 & 7.00 & 1.50 & .7 & .700 & 1,000 & $N$ & N \\
\hline 435687 & $\begin{array}{llll}65 & 42 & 47\end{array}$ & 1463737 & 7.00 & 2.00 & 3.0 & .700 & 1,000 & $N$ & $\mathrm{H}$ \\
\hline 435688 & 65487 & $146 \quad 2927$ & 7.00 & 1.50 & 3.0 & .700 & 1,500 & $N$ & $N$ \\
\hline 435689 & 655013 & 1463225 & 5.00 & 1.50 & .7 & .500 & 1,500 & 6.5 & $N$ \\
\hline
\end{tabular}


TABLE 2. - RESUL TS OF SPECTROGRAPHIC ANALYSES OF NURE STREAM-SEDIMENT SAMPLES FROM THE LIVENGOOD

AND WESTERN $1 / 3$ OF THE CIRCLE QUADRANGLES, ALASKA-Continued

\begin{tabular}{|c|c|c|c|c|c|c|c|c|c|c|}
\hline Satiple & $\begin{array}{c}\text { B-pp画 } \\
5\end{array}$ & $\begin{array}{c}B a-p p \text { 西 } \\
s\end{array}$ & $\begin{array}{c}\text { Be-ppit } \\
5\end{array}$ & $\begin{array}{c}B i-p p i n \\
5\end{array}$ & $\begin{array}{c}\text { Co-ppl } \\
5\end{array}$ & $\begin{array}{c}C r-p p \text { 醉 } \\
5\end{array}$ & $\begin{array}{c}\text { Cu-ppin } \\
s\end{array}$ & $\begin{array}{l}\text { La-ppa } \\
5\end{array}$ & $\begin{array}{c}\text { Mo-ppi } \\
5\end{array}$ & $\begin{array}{c}\text { Wb-pp而 } \\
5\end{array}$ \\
\hline 435645 & 100 & 1,500 & 1.5 & $N$ & 15 & 150 & 20 & 70 & $<5$ & 20 \\
\hline 435646 & 150 & 2,000 & 1.5 & $<10$ & 20 & 300 & 30 & 70 & $<5$ & $<20$ \\
\hline 435647 & 100 & 1,500 & 1.0 & $N$ & 15 & 100 & 15 & 70 & $<5$ & $<20$ \\
\hline 435648 & 100 & 1,500 & 1.0 & $N$ & 15 & 150 & 20 & 70 & $<5$ & $<20$ \\
\hline 435649 & 100 & 1,000 & $\langle 1.0$ & $N$ & 20 & 150 & 15 & 50 & $\langle 5$ & $N$ \\
\hline 435650 & 200 & 1,500 & 1.0 & $N$ & 20 & 150 & 20 & 70 & $\langle 5$ & $N$ \\
\hline 435651 & $N$ & 300 & 1.0 & $<10$ & $N$ & $<10$ & 30 & $N$ & $\mathrm{~N}$ & 20 \\
\hline 435652 & 150 & 1,500 & 1.0 & $N$ & 30 & 20 & 50 & 70 & $\langle 5$ & $<20$ \\
\hline 435653 & 200 & 1,500 & 1.0 & $H$ & 30 & 300 & 5 & 50 & $\langle 5$ & $<20$ \\
\hline 435654 & 150 & 1,500 & 1.0 & $N$ & 20 & 300 & 50 & 70 & 5 & $\langle 20$ \\
\hline 435655 & 100 & 1,500 & $\langle 1.0$ & $N$ & 20 & 200 & 20 & 50 & $N$ & $<20$ \\
\hline 435656 & 100 & 1,000 & $(1.0$ & $N$ & 10 & 100 & 15 & 50 & $N$ & $<20$ \\
\hline 435657 & 150 & 1,500 & 1.0 & $N$ & 15 & 150 & 30 & 50 & $N$ & $<20$ \\
\hline 435658 & 200 & 1,000 & 1.0 & $N$ & 15 & 100 & 30 & 70 & $N$ & $<20$ \\
\hline 435659 & 70 & 1,000 & 2.0 & $N$ & 15 & 150 & 15 & 70 & $N$ & $<20$ \\
\hline 435660 & 100 & 1,000 & 1.0 & $N$ & 15 & 200 & 15 & 50 & $N$ & $<20$ \\
\hline 435661 & 100 & 1,500 & 1.5 & $N$ & 15 & 200 & 50 & 70 & $N$ & 20 \\
\hline 435662 & 100 & 2,000 & 1.0 & $N$ & 20 & 200 & 30 & 50 & $\langle 5$ & $\langle 20$ \\
\hline 435663 & 70 & 1,000 & $\langle 1.0$ & $N$ & 15 & 150 & 30 & 50 & $N$ & $<20$ \\
\hline 435664 & 150 & 2,000 & 1.5 & $N$ & 20 & 150 & 50 & 50 & $<5$ & 20 \\
\hline 435665 & 70 & 1,500 & $\{1.0$ & $N$ & 7 & 200 & 50 & 70 & 10 & $<20$ \\
\hline 435666 & 70 & 2,000 & $\ll 1.0$ & $N$ & 7 & 150 & 50 & 30 & $<5$ & $<20$ \\
\hline 4355667 & 70 & 2,000 & 1.0 & $N$ & 15 & 100 & 30 & 100 & 7 & 20 \\
\hline 435668 & $N$ & 100 & $\langle 1.0$ & $N$ & $N$ & 40 & 45 & $N$ & $N$ & $N$ \\
\hline 435669 & 70 & 2,000 & $\$ 1.0$ & $N$ & 15 & 200 & 30 & 50 & $N$ & $<20$ \\
\hline 435670 & 100 & 3,000 & 1.0 & $N$ & 30 & 150 & 70 & 70 & 7 & $<20$ \\
\hline 435671 & 100 & 2,000 & 1.0 & $N$ & 20 & 200 & 50 & 50 & 45 & $<20$ \\
\hline 435672 & 70 & 2,000 & $\langle 1.0$ & $N$ & 15 & 150 & 50 & 30 & N & $<20$ \\
\hline 435673 & 10 & 300 & 1.0 & $N$ & $N$ & 110 & 15 & $N$ & $N$ & $N$ \\
\hline 435674 & 100 & 2,000 & 1.0 & $N$ & 20 & 300 & 50 & 50 & $N$ & 20 \\
\hline 435675 & 100 & 2,000 & 1.0 & $N$ & 15 & 200 & 30 & 50 & N & $<20$ \\
\hline 435676 & 100 & 1,500 & 1.0 & $N$ & 20 & 150 & 20 & 50 & $N$ & $<20$ \\
\hline 435677 & 100 & 2,000 & 1.0 & $N$ & 20 & 200 & 50 & 50 & $N$ & 30 \\
\hline 435678 & 100 & 2,000 & 1.0 & $\mathbf{N}$ & 20 & 200 & 30 & 50 & $N$ & $N$ \\
\hline 435679 & 70 & 1,000 & 1.0 & $N$ & 15 & 70 & 20 & 50 & N & $N$ \\
\hline 435680 & 50 & 1,000 & $\langle 1.0$ & $N$ & 15 & 100 & 20 & 50 & $N$ & $<20$ \\
\hline 435681 & 100 & 1,000 & 1.0 & $N$ & 15 & 150 & 30 & 50 & $N$ & 20 \\
\hline 435682 & 50 & 1,000 & $\langle 1.0$ & $N$ & 15 & 150 & 50 & 30 & $N$ & 20 \\
\hline 435683 & 70 & 1,000 & $\$ 1.0$ & $N$ & 10 & 70 & 15 & 50 & $N$ & $N$ \\
\hline 435684 & 50 & 1,000 & 1.0 & $N$ & 15 & 70 & 20 & 30 & $n$ & $<20$ \\
\hline 435685 & 50 & 700 & $\langle 1.0$ & $N$ & 15 & 100 & 30 & 50 & $N$ & $N$ \\
\hline 435686 & 70 & 700 & 11.0 & $N$ & 10 & 100 & 15 & 50 & $N$ & $N$ \\
\hline 435687 & 50 & 1,000 & $N$ & $N$ & 15 & 500 & 20 & 20 & $<5$ & $H$ \\
\hline 435688 & 50 & 1,000 & 11.0 & $N$ & 15 & 100 & 20 & 30 & $N$ & $N$ \\
\hline 435689 & 50 & 1,000 & $(1.0$ & $N$ & 10 & 100 & 10 & 30 & $N$ & $N$ \\
\hline
\end{tabular}


TABLE 2.--RESULTS OF SPECTROGRAPHIC ANALYSES OF NURE STREAM-SEDIMENT SAMPLES FRDM THE LIVENGOOD

AND WESTERN $1 / 3$ OF THE CIRCLE QUADRANGLES, ALASKA--Continued

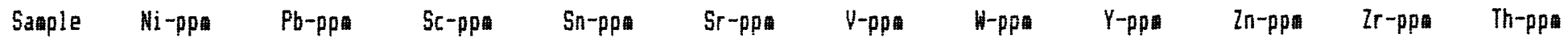

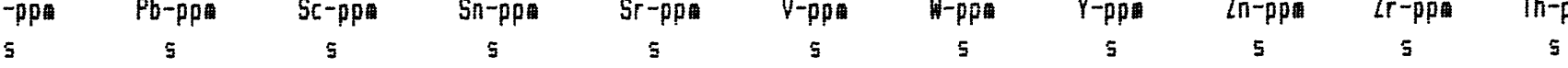

\begin{tabular}{|c|c|c|c|c|c|c|c|c|c|c|}
\hline 435645 & 50 & 30 & 20 & 15 & 150 & 150 & $N$ & 50 & $N$ & 300 \\
\hline 435646 & 70 & 50 & 30 & 20 & 200 & 200 & $N$ & 50 & $N$ & 300 \\
\hline 435647 & 30 & 30 & 15 & $N$ & 200 & 150 & $N$ & 30 & $N$ & 700 \\
\hline 435648 & 30 & 30 & 20 & $N$ & 200 & 150 & $N$ & 50 & $N$ & 500 \\
\hline 435649 & 30 & 30 & 10 & $N$ & 100 & 70 & $N$ & 20 & $N$ & 200 \\
\hline 435650 & 50 & 50 & 20 & 10 & 200 & 150 & $N$ & 50 & $N$ & 700 \\
\hline 435651 & 5 & $<10$ & $\langle 5$ & $N$ & $N$ & 30 & $N$ & 15 & $N$ & 50 \\
\hline 435652 & 70 & 50 & 30 & 20 & 200 & 200 & $N$ & 50 & $N$ & 200 \\
\hline 435653 & 70 & 30 & 20 & $<10$ & 200 & 200 & $N$ & 50 & $N$ & 300 \\
\hline 435654 & 70 & 50 & 20 & 10 & 200 & 150 & $N$ & 50 & $N$ & 300 \\
\hline 435655 & 50 & 30 & 20 & $N$ & 200 & 150 & $N$ & 50 & $N$ & 300 \\
\hline 435656 & 30 & 30 & 15 & $N$ & 200 & 100 & $N$ & 30 & $N$ & 500 \\
\hline 435657 & 30 & 30 & 20 & $N$ & 200 & 150 & $N$ & 50 & $<200$ & 700 \\
\hline 435658 & 30 & 30 & 20 & $N$ & 200 & 150 & $N$ & 70 & $N$ & 500 \\
\hline 435659 & 20 & 70 & 15 & 15 & 200 & 150 & $N$ & 50 & $\measuredangle 200$ & 500 \\
\hline 435660 & 30 & 30 & 20 & 10 & 200 & 150 & $N$ & 50 & $N$ & 700 \\
\hline 435661 & 30 & 50 & 20 & $N$ & 300 & 150 & $N$ & 50 & $N$ & 500 \\
\hline 435662 & 30 & 30 & 15 & 10 & 300 & 200 & $N$ & 50 & $N$ & 500 \\
\hline 435463 & 50 & 20 & 15 & $N$ & 200 & 150 & $N$ & 50 & $N$ & 300 \\
\hline 435664 & 70 & 500 & 20 & $<10$ & 100 & 200 & $N$ & 30 & 200 & 200 \\
\hline 435665 & 30 & 20 & 10 & $N$ & 100 & 200 & $N$ & 30 & $N$ & 300 \\
\hline 435666 & 30 & 20 & 20 & $N$ & 150 & 300 & H & 50 & $N$ & 300 \\
\hline 435667 & 30 & 30 & 20 & $N$ & 200 & 300 & $N$ & 70 & $N$ & 700 \\
\hline 435668 & $N$ & $N$ & $N$ & N & $N$ & 15 & $N$ & 10 & N & $N$ \\
\hline 435669 & 30 & 30 & 20 & $N$ & 500 & 200 & $N$ & 50 & $N$ & 300 \\
\hline 435670 & 70 & 30 & 20 & $N$ & 200 & 500 & $N$ & 50 & $N$ & 300 \\
\hline 435671 & 50 & 50 & 20 & $N$ & 200 & 200 & $N$ & 30 & 200 & 300 \\
\hline 435672 & 50 & 30 & 20 & $N$ & 300 & 200 & $N$ & 30 & $N$ & 200 \\
\hline 435673 & 7 & $<10$ & $\langle 5$ & $N$ & $<100$ & 30 & $M$ & 10 & $N$ & 70 \\
\hline 435674 & 70 & 50 & 20 & N & 200 & 200 & $N$ & 50 & $N$ & 300 \\
\hline 435675 & 50 & 30 & 20 & $N$ & 200 & 200 & $N$ & 50 & $<200$ & 500 \\
\hline 435676 & 50 & 30 & 15 & N & 200 & 150 & $N$ & 30 & $<200$ & 300 \\
\hline 435677 & 50 & 50 & 20 & $N$ & 200 & 200 & $N$ & 50 & $<200$ & 150 \\
\hline 435678 & 50 & 50 & 20 & $N$ & 200 & 200 & $N$ & 50 & $<200$ & 200 \\
\hline 435679 & 50 & 20 & 10 & $N$ & 200 & 150 & $N$ & 30 & 200 & 200 \\
\hline 435680 & 30 & 20 & 10 & $N$ & 150 & 150 & $N$ & 30 & $<200$ & 200 \\
\hline 435681 & 50 & 20 & 10 & $N$ & 100 & 200 & $N$ & 20 & $N$ & 150 \\
\hline 435682 & 50 & 20 & 10 & $N$ & 150 & 150 & $N$ & 50 & N & 200 \\
\hline 435683 & 30 & 50 & 10 & $\$ 10$ & 200 & 150 & $N$ & 30 & $N$ & 300 \\
\hline 435684 & 30 & 15 & 10 & $N$ & 300 & 150 & $N$ & 30 & $H$ & 200 \\
\hline 435685 & 50 & 20 & 7 & N & 150 & 150 & $N$ & 20 & $N$ & 300 \\
\hline 435686 & 50 & 30 & 10 & 10 & 100 & 200 & $N$ & 20 & $N$ & 200 \\
\hline 435687 & 100 & 15 & 7 & N & 150 & 200 & $N$ & 20 & $N$ & 300 \\
\hline 435688 & 30 & 20 & 7 & $N$ & 200 & 200 & $N$ & 20 & H & 500 \\
\hline 435689 & 20 & 15 & 7 & 10 & 100 & 200 & $N$ & 15 & $N$ & 200 \\
\hline
\end{tabular}




\begin{tabular}{|c|c|c|c|c|c|c|c|c|c|}
\hline Saliple & Latitude & Longitude & $\begin{array}{c}\text { Fe-pct. } \\
s\end{array}$ & $\begin{array}{c}\mathrm{Hg}_{\mathrm{g}}-\mathrm{pct} . \\
\mathrm{s}\end{array}$ & $\underset{5}{c a-p c t .}$ & $\begin{array}{c}\text { Ti-pet. } \\
5\end{array}$ & $\begin{array}{l}\text { Mn-ppi } \\
5\end{array}$ & $\begin{array}{l}\mathrm{Ag}-\mathrm{pp} \text { if } \\
5\end{array}$ & $\begin{array}{c}\text { As-ppin } \\
5\end{array}$ \\
\hline 435690 & 65512 & 1463026 & 5.00 & 1.50 & 1.5 & .700 & 700 & N & N \\
\hline 435691 & 655045 & $146 \quad 2832$ & 5.00 & 1.50 & 2.0 & .500 & 1,000 & $N$ & N \\
\hline 435692 & $6552 \quad 8$ & 1463121 & 5.00 & 1.50 & 1.0 & .500 & 1,000 & $N$ & N \\
\hline 435693 & 655438 & 1463218 & 5.00 & 1.50 & 2.0 & .500 & 1,000 & N & $N$ \\
\hline 435694 & 654915 & 1463625 & 5.00 & 1.50 & 2.0 & .700 & 1,500 & $N$ & N \\
\hline 435695 & 654941 & $14645 \quad 5$ & 5.00 & 1.50 & .7 & .700 & 1,000 & $N$ & N \\
\hline 435696 & 654950 & $146 \quad 4255$ & 5.00 & 1.50 & .5 & .700 & 1,500 & N & N \\
\hline 435697 & 654743 & 1463755 & 2.00 & 1.00 & 1.0 & .300 & 700 & N & N \\
\hline 435698 & 65483 & $14637 \quad 17$ & 3.00 & 1.50 & 1.5 & .500 & 1,000 & N & $n$ \\
\hline 435699 & 654940 & $14658 \quad 23$ & 5.00 & 1.00 & 1.0 & .500 & 300 & 1.0 & N \\
\hline 435700 & 65473 & $146 \quad 4250$ & 2.00 & 1.00 & 2.0 & .500 & 500 & 6.5 & N \\
\hline 435701 & 65425 & $146 \quad 3714$ & 7.00 & 1.50 & 1.5 & .700 & 1,000 & $N$ & N \\
\hline 435702 & 654012 & $146 \quad 34 \quad 11$ & 7.00 & 1.00 & .3 & .200 & 1,000 & $<.5$ & N \\
\hline 435703 & 353955 & 1463338 & 5.00 & 1.50 & .3 & .500 & 1,000 & $<.5$ & N \\
\hline 435704 & 65375 & 1462922 & 5.00 & 1.50 & 1.0 & .700 & 1,000 & $N$ & $N$ \\
\hline 435705 & 653722 & $14630 \quad 8$ & 7.00 & 1.50 & .5 & .700 & 1,000 & N & N \\
\hline $43570 \mathrm{~s}$ & 653643 & $146 \quad 3235$ & 7.00 & 1.50 & 1.0 & .700 & 500 & N & $H$ \\
\hline 435707 & 653629 & 1463153 & 10.00 & 1.50 & 1.0 & 1.000 & 500 & N & N \\
\hline 435708 & 653549 & 1463223 & 7.00 & 1.50 & .7 & 1.000 & 1,000 & N & N \\
\hline 435709 & $6536 \quad 2$ & $146 \quad 3314$ & 7.00 & 1.50 & .7 & 1.000 & 1,000 & N & N \\
\hline 435710 & 653410 & $146 \quad 27 \quad 7$ & 7.00 & 1.50 & 1.0 & 1.000 & 2,000 & N & $N$ \\
\hline 435711 & $6534 \quad 4$ & $146 \quad 2 b \quad 17$ & 10.00 & 1.50 & 1.0 & 1.000 & 3,000 & N & N \\
\hline 435712 & 653217 & 1462828 & 5.00 & 1.50 & 1.0 & .700 & 1,000 & $N$ & N \\
\hline 435713 & 653225 & 146315 & 7.00 & 1.00 & .5 & 1.000 & 700 & N & N \\
\hline 435714 & 653023 & 146310 & 7.00 & 1.50 & 1.0 & 1.000 & 1,500 & N & $N$ \\
\hline 435715 & $6530 \quad 6$ & 1463016 & 10.00 & 1.00 & .5 & .500 & 5,000 & $N$ & N \\
\hline 435716 & $6528 \quad 49$ & 1463225 & 15.00 & 2.00 & .7 & 1.000 & 2,000 & $N$ & N \\
\hline 435717 & 652735 & 1463454 & 7.00 & 1.50 & 1.0 & 1.000 & 1,000 & N & N \\
\hline 435718 & 652810 & 1463524 & 7.00 & 1.50 & 1.0 & 1.000 & 1,000 & $N$ & $H$ \\
\hline 435719 & 652627 & $146 \quad 3420$ & 7.00 & 1.50 & 1.5 & .700 & 700 & N & $N$ \\
\hline 435720 & 652432 & 1463346 & 7.00 & 1.50 & 1.0 & .700 & 700 & 6.5 & N \\
\hline 435721 & 652454 & $146 \quad 32 \quad 27$ & 5.00 & 1.50 & 1.5 & .700 & 500 & $N$ & N \\
\hline 435722 & 652215 & $14 b \quad 34 \quad 10$ & 5.00 & 1.50 & .3 & .700 & 1,000 & $N$ & $N$ \\
\hline 435723 & 652223 & $146 \quad 34 \quad 48$ & 5.00 & 1.50 & .5 & .700 & 700 & N & N \\
\hline 435724 & 652052 & $146 \quad 3458$ & 7.00 & 1.50 & .7 & 1.000 & 1,000 & $H$ & $N$ \\
\hline 435725 & 651828 & 1463516 & 7.00 & 1.50 & 1.5 & 1.000 & 700 & N & N \\
\hline $43572 b$ & $6518 \quad 8$ & $14636 \quad 6$ & 7.00 & 1.50 & 2.0 & 1.000 & 700 & $N$ & N \\
\hline 435727 & 651318 & 1463553 & 10.00 & 1.50 & .3 & .700 & 300 & 6.5 & N \\
\hline 435728 & 651225 & 146336 & 10.00 & 1.50 & 2.0 & 1.000 & 1,000 & $N$ & $N$ \\
\hline 435729 & 65916 & $14 b 3550$ & 7.00 & 1.50 & 2.0 & 1.000 & 700 & N & $N$ \\
\hline 435730 & 65933 & 1463517 & 10.00 & 1.50 & 1.0 & 1.000 & 1,000 & N & $N$ \\
\hline 435731 & 6593 & $146 \quad 34 \quad 18$ & 15.00 & 1.50 & 2.0 & .700 & 500 & N & N \\
\hline 435732 & 65835 & 1463435 & 7.00 & 1.50 & 2.0 & 1.000 & 700 & $H$ & $H$ \\
\hline 435733 & 6579 & 146313 & 5.00 & 1.00 & 2.0 & .700 & 700 & N & N \\
\hline 435734 & 65715 & $146 \quad 32 \quad 3$ & 3.00 & 1.00 & 2.0 & .500 & 500 & $N$ & N \\
\hline
\end{tabular}


TABLE 2. - PESULTS OF SPECTROSRAPHIC ANALYSES OF NURE STREAM-SEDIMENT SAMPLES FROM THE LIVENGDOD

AND WESTERN $1 / 3$ OF THE CIRCLE QUADRANGLES, ALASKA--ContinURd

\begin{tabular}{|c|c|c|c|c|c|c|c|c|c|c|}
\hline Sample & $\begin{array}{c}\text { B-ppm } \\
5\end{array}$ & $\begin{array}{c}\text { Ba-ppi } \\
5\end{array}$ & $\begin{array}{c}\mathrm{Be}-\mathrm{pp} \text {. } \\
\mathrm{s}\end{array}$ & $\begin{array}{c}\text { Bi-ppil } \\
5\end{array}$ & $\begin{array}{c}\text { Co-ppm } \\
s\end{array}$ & $\begin{array}{c}\text { Cr-ppi } \\
5\end{array}$ & 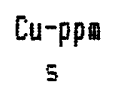 & $\begin{array}{c}\text { La-ppi } \\
5\end{array}$ & $\begin{array}{c}\text { Mo-pp田 } \\
5\end{array}$ & $\begin{array}{c}\text { Nb-ppm } \\
s\end{array}$ \\
\hline 435690 & 50 & 1,000 & 1.0 & $N$ & 15 & 70 & 20 & 50 & $N$ & $<20$ \\
\hline 435691 & 50 & 1,000 & 1.0 & $N$ & 10 & 70 & 15 & 70 & $N$ & $<20$ \\
\hline 435692 & 70 & 700 & 1.0 & $N$ & 15 & $100^{\circ}$ & 20 & 70 & $N$ & $<20$ \\
\hline 435693 & 50 & 1,000 & $\$ 1.0$ & $N$ & 10 & 100 & 15 & 30 & $N$ & 20 \\
\hline 435694 & 50 & 1,000 & $N$ & $N$ & 15 & 100 & 20 & 70 & $N$ & $<20$ \\
\hline 435695 & 100 & 700 & $\$ 1.0$ & $N$ & 15 & 100 & 20 & 50 & $N$ & $<20$ \\
\hline 435696 & 100 & 700 & 41.0 & $N$ & 15 & 100 & 20 & 50 & $<5$ & $\langle 20$ \\
\hline 435697 & 20 & 700 & $\langle 1.0$ & $N$ & 15 & 30 & 15 & $N$ & $N$ & $N$ \\
\hline 435698 & 70 & 700 & $\langle 1.0$ & $N$ & 15 & 70 & 20 & 50 & $N$ & $<20$ \\
\hline 435699 & 100 & 2,000 & $\$ 1.0$ & $N$ & 5 & 100 & 30 & 70 & 5 & 20 \\
\hline 435700 & 50 & 700 & H & $N$ & 10 & 100 & 15 & 50 & $N$ & $N$ \\
\hline 435701 & 100 & 1,000 & 1.5 & $N$ & 15 & 150 & 30 & 70 & $N$ & 20 \\
\hline 435702 & 50 & 500 & 3.0 & $N$ & 7 & 70 & 20 & 70 & $<5$ & $<20$ \\
\hline 435703 & 100 & 700 & 5.0 & $N$ & 10 & 100 & 20 & 150 & 7 & 20 \\
\hline 435704 & 100 & 1,000 & 1.0 & $N$ & 15 & 150 & 20 & 50 & N & $<20$ \\
\hline 435705 & 100 & 1,000 & 1.5 & $N$ & 15 & 100 & 20 & 70 & $N$ & 20 \\
\hline 435706 & 100 & 1,000 & 1.0 & $N$ & 15 & $100^{\circ}$ & 30 & 100 & N & $<20$ \\
\hline 435707 & 150 & 1,000 & 1.0 & $N$ & 15 & 150 & 20 & 100 & $N$ & $\langle 20$ \\
\hline 435708 & 100 & 700 & 1.0 & $N$ & 15 & 100 & 20 & 70 & 7 & $<20$ \\
\hline 435709 & 150 & 700 & 1.0 & $N$ & 15 & 100 & 20 & 70 & $N$ & 20 \\
\hline 435710 & 100 & 1,000 & 2.0 & $N$ & 20 & 100 & 15 & 100 & $H$ & $<20$ \\
\hline 435711 & 150 & 100 & 2.0 & $N$ & 20 & 150 & 20 & 70 & $<5$ & 20 \\
\hline 435712 & 70 & 1,000 & 1.0 & $n$ & 10 & 70 & 20 & 50 & $\mathrm{~N}$ & $N$ \\
\hline 435713 & 150 & 700 & 1.5 & $N$ & 10 & 70 & 15 & 70 & N & 20 \\
\hline 435714 & 150 & 1,000 & 1.0 & $N$ & 15 & 100 & 20 & 70 & N & $<20$ \\
\hline 435715 & 100 & 700 & 3.0 & $N$ & 30 & 50 & 15 & 70 & $N$ & N \\
\hline 435716 & 150 & 1,000 & 1.0 & $N$ & 10 & 150 & 15 & $N$ & $N$ & $<20$ \\
\hline 435717 & 100 & 1,000 & 1.5 & $N$ & 15 & 100 & 20 & 70 & N & 20 \\
\hline 435718 & 150 & 1,000 & 2.0 & $N$ & 15 & 70 & 20 & 70 & $N$ & $<20$ \\
\hline 435719 & 150 & 700 & 2.0 & $N$ & 10 & 100 & 15 & 70 & $N$ & $\langle 20$ \\
\hline 435720 & 150 & 700 & 5.0 & $\longleftrightarrow 10$ & 15 & 100 & 15 & 50 & 5 & $<20$ \\
\hline 435721 & 200 & 700 & 15.0 & $\$ 10$ & 10 & 150 & 15 & 70 & $<5$ & $<20$ \\
\hline 435722 & 200 & 500 & 2.0 & $N$ & 20 & 50 & 15 & 50 & $N$ & $<20$ \\
\hline 435723 & 100 & 700 & 1.5 & $N$ & 15 & 100 & 20 & 50 & H & $<20$ \\
\hline 435724 & 100 & 1,000 & 1.5 & $N$ & 15 & 100 & 20 & 100 & $N$ & 20 \\
\hline 435725 & 100 & 700 & 1.0 & $H$ & 15 & 100 & 20 & 50 & $N$ & $<20$ \\
\hline 435726 & 100 & 700 & $\langle 1.0$ & $N$ & 15 & 100 & 20 & 50 & N & $<20$ \\
\hline 435727 & 150 & 1,000 & 1.5 & $N$ & 15 & 150 & 30 & 70 & $N$ & 20 \\
\hline 435728 & 100 & 1,000 & 1.0 & $M$ & 20 & 150 & 50 & 70 & $N$ & $<20$ \\
\hline 435729 & 150 & 700 & 1.0 & $N$ & 10 & 70 & 10 & 50 & N & 20 \\
\hline 435730 & 150 & 1,000 & 1.0 & $N$ & 15 & 100 & 15 & 50 & N & 20 \\
\hline 435731 & 100 & 1,000 & 1.0 & 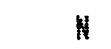 & 15 & 100 & 20 & 70 & H & 20 \\
\hline 435732 & 150 & 1,000 & 1.0 & $N$ & 15 & 100 & 15 & 50 & $N$ & $<20$ \\
\hline 435733 & 50 & 700 & 1.0 & $N$ & 10 & 70 & 20 & 50 & N & N \\
\hline 435734 & 50 & 700 & 1.0 & N & 10 & 50 & 15 & 50 & $\langle 5$ & $N$ \\
\hline
\end{tabular}


TABLE 2. - RESULTS OF SFECTRDGRAPHIC AMALYSES OF NURE STREAM-SEDIMENT SAMPLES FROM THE LIVENGOOD

AND WESTERN $1 / 3$ OF THE CIRCLE QUADRANGLES, ALASKA-Continued

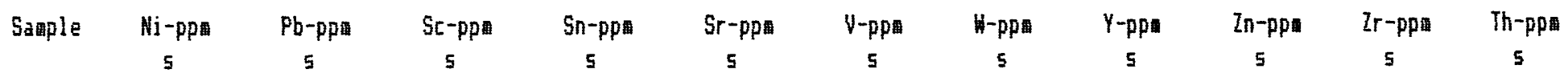

\begin{tabular}{|c|c|c|c|c|c|c|c|c|c|c|c|}
\hline 435690 & 30 & 50 & 10 & $N$ & 200 & 150 & $N$ & 20 & $N$ & 200 & $N$ \\
\hline 435691 & 30 & 15 & 10 & $N$ & 300 & 150 & $N$ & 30 & $N$ & 300 & N \\
\hline 435692 & 30 & 15 & 10 & $N$ & 200 & 150 & $N$ & 20 & $N$ & 300 & V \\
\hline 435693 & 30 & 15 & 7 & $N$ & 200 & 150 & $N$ & 30 & $N$ & 300 & N \\
\hline 435694 & 30 & 50 & 10 & $N$ & 200 & 150 & $\|$ & 20 & $N$ & 300 & $N$ \\
\hline 435695 & 30 & 20 & 7 & 10 & 150 & 150 & $N$ & 20 & $N$ & 150 & $N$ \\
\hline 435696 & 50 & 20 & 10 & 10 & 150 & 150 & $N$ & 30 & $N$ & 200 & $N$ \\
\hline 435697 & 50 & 15 & 7 & $N$ & 150 & 100 & $N$ & 20 & $\mathrm{~N}$ & 100 & H \\
\hline 435698 & 30 & 20 & 10 & $N$ & 200 & 100 & $N$ & 30 & $N$ & 200 & $N$ \\
\hline 435699 & 30 & 20 & 10 & $N$ & 150 & 300 & $N$ & 20 & N & 200 & $N$ \\
\hline 435700 & 30 & 10 & 7 & $N$ & 200 & 100 & $N$ & 20 & $N$ & 150 & N \\
\hline 435701 & 50 & 30 & 15 & $<10$ & 200 & 150 & $N$ & 50 & $N$ & 500 & $N$ \\
\hline 435702 & 20 & 50 & 10 & 20 & $N$ & 70 & $N$ & 70 & $N$ & 300 & $N$ \\
\hline 435703 & 30 & 100 & 10 & 50 & $<100$ & 100 & $N$ & 70 & 200 & 700 & 100 \\
\hline 435704 & 30 & 30 & 15 & $N$ & 150 & 150 & $H$ & 50 & $N$ & 500 & $N$ \\
\hline 435705 & 50 & 30 & 15 & $N$ & 100 & 150 & $N$ & 30 & $N$ & 500 & $N$ \\
\hline 435706 & 50 & 20 & 15 & $N$ & 150 & 150 & $N$ & 50 & $N$ & 300 & $N$ \\
\hline 435707 & 30 & 30 & 15 & $N$ & 150 & 150 & $N$ & 50 & $N$ & 500 & $N$ \\
\hline 435708 & 30 & 30 & 15 & $N$ & 100 & 150 & $N$ & 30 & $N$ & 500 & $N$ \\
\hline 435709 & 30 & 100 & 15 & N & 100 & 150 & $N$ & 50 & N & 1,000 & $N$ \\
\hline 435710 & 30 & 30 & 10 & $\langle 10$ & 150 & 150 & $N$ & 30 & $N$ & 300 & $N$ \\
\hline 435711 & 50 & 50 & 15 & 10 & 150 & 150 & $N$ & 50 & $N$ & 300 & $N$ \\
\hline 435712 & 30 & 20 & 10 & $N$ & 100 & 150 & $N$ & 30 & $N$ & 300 & $N$ \\
\hline 435713 & 30 & 20 & 7 & $N$ & 150 & 100 & $N$ & 50 & N & 700 & $N$ \\
\hline 435714 & 30 & 30 & 10 & $N$ & 150 & 100 & $N$ & 50 & $N$ & 1,000 & N \\
\hline 435715 & 30 & 20 & 7 & $N$ & 100 & 100 & $N$ & 50 & $N$ & 200 & $N$ \\
\hline 435716 & 30 & 50 & 77 & $N$ & $<100$ & 150 & $N$ & 20 & $N$ & 500 & $N$ \\
\hline 435717 & 30 & 30 & 15 & 40 & 200 & 150 & $N$ & 50 & $N$ & 500 & $N$ \\
\hline 435718 & 30 & 30 & 10 & 10 & 150 & 100 & $N$ & 50 & $N$ & 700 & $N$ \\
\hline 435719 & 20 & 30 & 10 & 20 & 150 & 100 & $N$ & 70 & $N$ & 300 & N \\
\hline 435720 & 30 & 30 & 10 & 10 & 150 & 100 & $N$ & 50 & $N$ & 200 & $N$ \\
\hline 435721 & 20 & 30 & 15 & 10 & 200 & 100 & $N$ & 70 & $N$ & 1,000 & H \\
\hline 435722 & 50 & 20 & 10 & $N$ & 100 & 100 & $N$ & 50 & $N$ & 700 & N \\
\hline 435723 & 50 & 20 & 15 & $N$ & 150 & 100 & $N$ & 30 & $N$ & 500 & $N$ \\
\hline 435724 & 50 & 30 & 15 & $N$ & 200 & 100 & $N$ & 50 & $N$ & 500 & $N$ \\
\hline 435725 & 30 & 20 & 15 & $N$ & 200 & 150 & $N$ & 30 & $N$ & 500 & $N$ \\
\hline 435726 & 30 & 20 & 15 & $N$ & 200 & 150 & $N$ & 30 & N & 300 & $N$ \\
\hline 435727 & 50 & 30 & 20 & $N$ & 200 & 150 & $N$ & 50 & $N$ & 200 & $N$ \\
\hline 435728 & 50 & 20 & 20 & $N$ & 200 & 150 & $N$ & 50 & N & 300 & $N$ \\
\hline 435729 & 20 & 20 & 15 & $N$ & 200 & 100 & $N$ & 30 & N & 1,000 & $N$ \\
\hline 435730 & 30 & 20 & 15 & $N$ & 200 & 150 & $N$ & 30 & $N$ & 500 & $N$ \\
\hline 435731 & 30 & 30 & 20 & $N$ & 200 & 150 & $N$ & 50 & N & 300 & $N$ \\
\hline 435732 & 30 & 20 & 15 & N & 200 & 150 & $N$ & 50 & $N$ & 500 & $N$ \\
\hline 435733 & 30 & 20 & 15 & $N$ & 200 & 150 & $N$ & 50 & $N$ & 300 & $N$ \\
\hline 435734 & 20 & 30 & 10 & N & 150 & 100 & $N$ & 30 & $N$ & 200 & $N$ \\
\hline
\end{tabular}


TAELE 2,--RESULTS OF SPECTROGRAPHIC AMALYSES OF MURE STREAM-SEDIMENT SAMPLES FROM THE LIVENG000 AND WESTERN $1 / 3$ OF THE CIRCLE QUADRAMGLES, ALASKA--Continued

\begin{tabular}{|c|c|c|c|c|c|c|c|c|c|}
\hline Sample & Latitude & Longitude & $\begin{array}{c}\text { Fe-pct. } \\
s\end{array}$ & $\begin{array}{c}\text { Mg-pet. } \\
s\end{array}$ & $\begin{array}{c}\text { Ca-pct. } \\
s\end{array}$ & $\begin{array}{c}\text { Ti-prt. } \\
s\end{array}$ & $\begin{array}{c}\text { Mn-ppon } \\
5\end{array}$ & $\begin{array}{l}A g-p p \text { I } \\
\vdots\end{array}$ & $\begin{array}{c}A s-p p \text { m } \\
5\end{array}$ \\
\hline 435735 & $65 \quad 6 \quad 6$ & $146 \quad 32 \quad 25$ & 10.00 & 2.00 & 3.0 & 1.000 & 1,000 & N & N \\
\hline 435736 & $65 \quad 6 \quad 2$ & $146 \quad 3325$ & 10.00 & 2.00 & 2.0 & 1.000 & 1,000 & $n$ & N \\
\hline 435737 & $65 \quad 4 \quad 0$ & 1463356 & 7.00 & 1.50 & 2.0 & 1.000 & 700 & N & N \\
\hline 435738 & 65329 & 1463320 & 7.00 & 1.50 & 2.0 & 1.000 & 1,000 & N & $N$ \\
\hline 435739 & 65318 & 146310 & 1.50 & .50 & .7 & .150 & 300 & 6.5 & $H$ \\
\hline 435740 & $65 \quad 353$ & 1462954 & .70 & .15 & .7 & .150 & 200 & $\langle .5$ & N \\
\hline 435741 & $65+19$ & 1462951 & .50 & .15 & .3 & .070 & 200 & 4.5 & $N$ \\
\hline 435742 & 65426 & 1462245 & 1.50 & 1.00 & 1.0 & .200 & 300 & 4.5 & H \\
\hline 435743 & $65 \quad 339$ & 1462119 & 1.00 & .50 & .2 & .150 & 200 & $<.5$ & $H$ \\
\hline 435753 & 65445 & 1465138 & 3.00 & 1.50 & 2.0 & .700 & 1,000 & N & $N$ \\
\hline 435754 & 65511 & 1465222 & 5.00 & 1.50 & 2.0 & 1.000 & 1,000 & N & $N$ \\
\hline 435755 & $65 \quad 657$ & 146543 & 5.00 & 2.00 & 3.0 & 1.000 & 1,000 & N & $H$ \\
\hline 435756 & 65656 & 1465218 & 5.00 & 2.00 & 3.0 & 1.000 & 1,000 & N & $H$ \\
\hline 435757 & $65 \quad 852$ & $146 \quad 9945$ & 5.00 & 2.00 & 2.0 & 1.000 & 700 & H & $N$ \\
\hline 435758 & 65933 & $14650 \quad 3$ & 5.00 & 2.00 & 3.0 & 1.000 & 2,000 & N & $N$ \\
\hline 435759 & 65129 & 1465018 & 5.00 & 1.50 & 1.0 & 1.000 & 700 & H & $N$ \\
\hline 435760 & 651238 & 1464958 & 5.00 & 1.50 & 1.5 & 1.000 & 1,000 & $N$ & $M$ \\
\hline 435761 & 651340 & 1465351 & 5.00 & 1.50 & .7 & .700 & 1,000 & N & $N$ \\
\hline 435762 & 65134 & 1465451 & 5.00 & 1.50 & 2.0 & .700 & 1,500 & $M$ & $N$ \\
\hline 435763 & 651324 & $146 \quad 52 \quad 15$ & 5.00 & 1.50 & 2.0 & 1.000 & 1,000 & N & $H$ \\
\hline 435764 & 651421 & 1465227 & 5.00 & 1.50 & 3.0 & 31.000 & 1,000 & $N$ & $N$ \\
\hline 435765 & 651614 & 1464929 & 7.00 & 2.00 & 2.0 & 1.000 & 500 & N & $n$ \\
\hline 435766 & 651550 & 1465130 & 5.00 & 2.00 & 2.0 & 1.000 & 500 & N & $N$ \\
\hline 435767 & 65160 & 1465218 & 10.00 & 3.00 & 2.0 & 1.000 & 700 & N & $N$ \\
\hline 435768 & 651633 & 1465236 & 7.00 & 2.00 & 1.5 & 1.000 & 700 & $N$ & $N$ \\
\hline 435770 & $6518 \quad 5$ & 146515 & 10.00 & 3.00 & 2.0 & 1.000 & 1,000 & M & $N$ \\
\hline 435771 & 651754 & $146 \quad 50 \quad 12$ & 5.00 & 2.00 & 2.0 & 1.000 & 1,000 & $N$ & $\mathrm{~N}$ \\
\hline 435772 & $6520 \quad 5$ & 1464944 & 5.00 & 1.50 & 2.0 & 1.000 & 500 & $n$ & $\mathrm{H}$ \\
\hline 435773 & 651951 & 1465025 & 7.00 & 1.50 & 1.5 & .500 & 700 & N & N \\
\hline 435774 & 652124 & 1465455 & 5.00 & 1.50 & .7 & 1.000 & 1,000 & N & $\mathrm{N}$ \\
\hline 435775 & 652128 & 1465241 & 5.00 & 1.50 & .7 & .700 & 700 & N & $\mathrm{H}$ \\
\hline 435776 & 65254 & 1465115 & 3.00 & 1.00 & 1.0 & 1.000 & 500 & N & $N$ \\
\hline 435777 & 652420 & 1465138 & 5.00 & 1.50 & 2.0 & 1.000 & 500 & $<.5$ & $N$ \\
\hline 4.35778 & 652634 & 1465259 & 3.00 & 1.50 & .5 & 1.000 & 700 & $\mathrm{H}$ & $n$ \\
\hline 435779 & 652750 & 1465439 & 3.00 & 1.50 & .5 & 1.000 & 500 & $\ddot{N}$ & $\mathrm{~N}$ \\
\hline 435780 & 652850 & 1465358 & 5.00 & 1.50 & 1.5 & 1.000 & 2,000 & $N$ & H \\
\hline 435781 & 652835 & $14651 \quad 37$ & 7.00 & 2.00 & .7 & .100 & 1,500 & N & $N$ \\
\hline 435782 & 653132 & $14651 \quad b$ & 5.00 & 1.50 & 1.0 & 1.000 & 1,000 & $N$ & $N$ \\
\hline 435783 & 65324 & $14650 \quad 16$ & 5.00 & 1.50 & .7 & 1.000 & 700 & $N$ & H \\
\hline 435785 & 653311 & 1465451 & 5.00 & 1.50 & 1.0 & 1.000 & 700 & N & $N$ \\
\hline 35786 & 653342 & 1465323 & 5.00 & 2.00 & 2.0 & 1.000 & 500 & N & N \\
\hline 335787 & 653432 & 1465041 & 3.00 & 1.00 & 1.0 & .500 & 700 & 6.5 & $n$ \\
\hline 135788 & 653637 & 146539 & 5.00 & 1.50 & 1.5 & 1.000 & 700 & $<.5$ & N \\
\hline 135789 & 653641 & 1465150 & 5.00 & 1.50 & 1.5 & 1.000 & 1,000 & 6.5 & N \\
\hline 135791 & 653737 & 1464928 & 3.00 & 1.00 & .5 & .700 & 1,500 & N & $N$ \\
\hline
\end{tabular}


TABLE 2.--RESLLLTS OF SPECTROGRAPHIC ANALYSES OF NURE STREAM-SEDIMENT SAMPLES FROK THE LIVENGDDd

AND WESTEFN $1 / 3$ OF THE CIRCLE QUADRANGLES, ALASKA--Continued

\begin{tabular}{|c|c|c|c|c|c|c|c|c|c|c|}
\hline Sa酯le & $\begin{array}{c}8-p p \text { q } \\
5\end{array}$ & $\begin{array}{c}\text { Ba-pp } \\
5\end{array}$ & $\begin{array}{c}\text { Be-ppi } \\
5\end{array}$ & $\begin{array}{c}\text { Bi-ppi } \\
5\end{array}$ & $\begin{array}{c}\text { Co-pp } \\
5\end{array}$ & $\begin{array}{c}\text { Cr-ppa } \\
s\end{array}$ & $\begin{array}{c}\text { Cu-ppin } \\
5\end{array}$ & $\begin{array}{c}\text { La-ppi } \\
5\end{array}$ & $\begin{array}{c}\text { Ho-ppon } \\
5\end{array}$ & $\begin{array}{c}\mathrm{Nb}-\mathrm{pp} \text { 的 } \\
5\end{array}$ \\
\hline 435735 & 100 & 1,000 & 1.0 & $N$ & 15 & 150 & 15 & 50 & $n$ & 20 \\
\hline 435736 & 100 & 1,000 & 1.0 & $n$ & 15 & 150 & 30 & 50 & $N$ & $<20$ \\
\hline 435737 & 70 & 1,000 & 1.0 & $N$ & 15 & 150 & 20 & 50 & $N$ & $\langle 20$ \\
\hline 435738 & 100 & 1,000 & 1.0 & $N$ & 15 & 100 & 20 & 70 & $N$ & $<20$ \\
\hline 435739 & 10 & 500 & 2.0 & $n$ & $N$ & 20 & 15 & $N$ & $N$ & $n$ \\
\hline 435740 & $<10$ & 300 & 2.0 & $N$ & 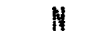 & 10 & 10 & 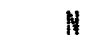 & $N$ & $N$ \\
\hline 435741 & <10 & 300 & 1.5 & $<10$ & N & $<10$ & 5 & $N$ & $N$ & N \\
\hline 435742 & 20 & 700 & 2.0 & 10 & $N$ & 30 & 15 & 50 & N & $N$ \\
\hline 435743 & 10 & 300 & 2.0 & 10 & $N$ & 20 & 10 & $N$ & $N$ & $N$ \\
\hline 435753 & 100 & 1,000 & 1.5 & $N$ & 10 & 70 & 15 & 50 & $<5$ & $N$ \\
\hline 435754 & 70 & 1,000 & 1.0 & $\mathrm{~N}$ & 10 & 50 & 15 & 50 & $N$ & 20 \\
\hline 435755 & 100 & 700 & 1.0 & $N$ & 15 & 150 & 15 & 50 & $N$ & 20 \\
\hline 435756 & 100 & 1,000 & 1.0 & $N$ & 20 & 150 & 20 & 70 & $<5$ & $<20$ \\
\hline 435757 & 100 & 1,000 & 1.0 & $N$ & 15 & 100 & 15 & 70 & $N$ & $<20$ \\
\hline 435758 & 20 & 1,000 & 1.5 & $N$ & 30 & 150 & 20 & 70 & $N$ & 20 \\
\hline 435759 & 150 & 700 & 1.0 & $N$ & 15 & 100 & 20 & 70 & N & 20 \\
\hline 435760 & 150 & 1,000 & 1.0 & $N$ & 20 & 100 & 20 & 70 & $N$ & 20 \\
\hline 435761 & 70 & 700 & 1.0 & 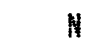 & 15 & 100 & 20 & 20 & 45 & 20 \\
\hline 435762 & 100 & 1,000 & 1.0 & $N$ & 15 & 100 & 15 & 30 & $H$ & 20 \\
\hline 435763 & 100 & 1,000 & 1.0 & $N$ & 20 & 150 & 20 & 50 & $N$ & $\langle 20$ \\
\hline 435764 & 150 & 700 & $\langle 1.0$ & $N$ & 15 & 100 & 15 & 50 & $N$ & 20 \\
\hline 435765 & 70 & 700 & 1.0 & $N$ & 20 & 100 & 20 & 50 & $N$ & $<20$ \\
\hline 435766 & 50 & 700 & 1.0 & $N$ & 20 & 70 & 50 & 20 & $n$ & $N$ \\
\hline 435767 & 50 & 500 & 11.0 & $N$ & 30 & 150 & 70 & 20 & $N$ & $N$ \\
\hline 435768 & 100 & 700 & 1.0 & N & 15 & 100 & 30 & 50 & $N$ & 20 \\
\hline 435770 & 70 & 500 & 41.0 & $N$ & 30 & 150 & 70 & 30 & $N$ & $N$ \\
\hline 435771 & 70 & 1,000 & 1.0 & $N$ & 15 & 150 & 30 & 50 & $N$ & 20 \\
\hline 435772 & 70 & 1,000 & 1.0 & $N$ & 10 & 150 & 15 & 50 & $N$ & $<20$ \\
\hline 435773 & 30 & 1,000 & 1.0 & $\mathrm{H}$ & 15 & 70 & 15 & 50 & $N$ & $N$ \\
\hline 435774 & 70 & 1,000 & 1.0 & $N$ & 10 & 70 & 15 & 50 & N & N \\
\hline 435775 & 70 & 1,000 & 1.0 & H & 15 & 100 & 15 & 50 & $n$ & $<20$ \\
\hline 435776 & 70 & 700 & 1.0 & $N$ & 10 & 100 & 10 & 70 & $N$ & $<20$ \\
\hline 435777 & 70 & 1,000 & 1.0 & $N$ & 15 & 150 & 15 & 50 & $N$ & $<20$ \\
\hline 435778 & 100 & 700 & 1.0 & N & 10 & 50 & 15 & 50 & $N$ & 20 \\
\hline 435779 & 100 & 700 & 1.0 & $N$ & 10 & 70 & 10 & 50 & $N$ & $<20$ \\
\hline 435780 & 70 & 1,000 & 1.0 & $N$ & 20 & 150 & 30 & 50 & $N$ & $<20$ \\
\hline 435781 & 150 & 1,000 & 2.0 & $N$ & 15 & 150 & 20 & 50 & $N$ & $<20$ \\
\hline 435782 & 100 & 1,000 & 1.5 & $N$ & 15 & 150 & 20 & 50 & $N$ & $<20$ \\
\hline 435783 & 100 & 700 & 3.0 & $N$ & 15 & 100 & 20 & 70 & $n$ & $<20$ \\
\hline 435765 & 100 & 1,500 & 1.0 & $N$ & 15 & 100 & 20 & 50 & $N$ & $<20$ \\
\hline 435786 & 100 & 1,500 & 1.5 & $N$ & 15 & 200 & 20 & 50 & $N$ & $\langle 20$ \\
\hline 435787 & 50 & 700 & 20.0 & $<10$ & 7 & 100 & 15 & 100 & 7 & 20 \\
\hline 435788 & 100 & 1,000 & 2.0 & $N$ & 15 & 150 & 20 & 70 & $<5$ & $<20$ \\
\hline 435789 & 100 & 1,000 & 3.0 & $N$ & 15 & 100 & 20 & 100 & $<5$ & 20 \\
\hline 435791 & 50 & 700 & 5.0 & 410 & 15 & 50 & 10 & 70 & $N$ & $<20$ \\
\hline
\end{tabular}


TABLE 2.--RESULTS OF SPECTRDGRAPHIC ANALYSES OF NURE STREAM-SEDIMENT SAMPLES FROM THE LIVENGODD

AND HESTERN $1 / 3$ OF THE CIRCLE QUADRANGLES, ALASKA--Continued

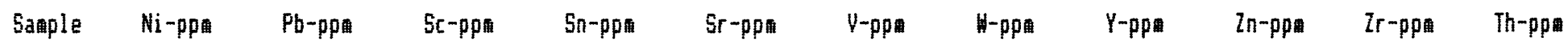

\begin{tabular}{|c|c|c|c|c|c|c|c|c|c|c|c|}
\hline 435735 & 50 & 30 & 15 & $N$ & 300 & 200 & $N$ & 30 & $N$ & 500 & $N$ \\
\hline 435736 & 30 & 50 & 15 & $N$ & 300 & 150 & $N$ & 50 & $N$ & 500 & $N$ \\
\hline 435737 & 30 & 20 & 15 & $N$ & 200 & 150 & N & 30 & $N$ & 300 & N \\
\hline 435738 & 30 & 30 & 15 & $N$ & 200 & 150 & $N$ & 50 & $N$ & 500 & N \\
\hline 435739 & 10 & 10 & 7 & $N$ & $<100$ & 70 & $N$ & 20 & $N$ & 70 & $N$ \\
\hline 435740 & 5 & $<10$ & 7 & $N$ & N & 50 & $N$ & 20 & $N$ & 70 & $N$ \\
\hline 435741 & $\langle 5$ & $<10$ & $\langle 5$ & $N$ & $N$ & 50 & $N$ & 10 & $N$ & 50 & N \\
\hline 435742 & 15 & 10 & 10 & $N$ & $\$ 100$ & 70 & $N$ & 30 & $N$ & 150 & N \\
\hline 435743 & 10 & 10 & 7 & $N$ & $N$ & 70 & $N$ & 15 & $N$ & 50 & N \\
\hline 435753 & 30 & 20 & 10 & $N$ & 200 & 100 & $n$ & 30 & $N$ & 300 & N \\
\hline 435754 & 30 & 20 & 7 & $N$ & 200 & 100 & N & 30 & $N$ & 500 & N \\
\hline 435755 & 50 & 20 & 15 & $N$ & 300 & 150 & $N$ & 30 & N & 500 & N \\
\hline 435756 & 50 & 30 & 20 & $N$ & 300 & 150 & $N$ & 50 & $N$ & 300 & N \\
\hline 435757 & 30 & 30 & 15 & $N$ & 300 & 150 & $N$ & 50 & $N$ & 300 & N \\
\hline 435758 & 100 & 20 & 20 & N & 300 & 150 & $N$ & 50 & $N$ & 300 & $N$ \\
\hline 435759 & 30 & 20 & 20 & $N$ & 200 & 100 & $N$ & 50 & $N$ & 300 & $N$ \\
\hline 435760 & 50 & 20 & 20 & $N$ & 200 & 100 & $N$ & 50 & $N$ & 300 & N \\
\hline 435761 & 50 & 20 & 15 & N & 150 & 100 & $N$ & 20 & $N$ & 150 & $N$ \\
\hline 435762 & 30 & 15 & 15 & $N$ & 200 & 100 & $N$ & 20 & $N$ & 500 & $N$ \\
\hline 435763 & 50 & 20 & 20 & $N$ & 200 & 100 & N & 30 & $N$ & 200 & N \\
\hline 435764 & 30 & 15 & 20 & $N$ & 200 & 100 & $N$ & 30 & $N$ & 500 & N \\
\hline 435765 & 50 & 15 & 20 & $N$ & 200 & 150 & $N$ & 30 & $N$ & 300 & $N$ \\
\hline 435766 & 50 & 10 & 20 & $N$ & 150 & 150 & $N$ & 20 & $N$ & 200 & $N$ \\
\hline 435767 & 70 & 20 & 20 & $N$ & 150 & 200 & $N$ & 20 & $N$ & 150 & N \\
\hline 435768 & 30 & 20 & 20 & $N$ & 150 & 150 & $N$ & 30 & $N$ & 300 & $N$ \\
\hline 435770 & 70 & 20 & 30 & $N$ & 100 & 200 & $N$ & 30 & $N$ & 200 & N \\
\hline 435771 & 30 & 20 & 15 & $N$ & 200 & 100 & N & 30 & $N$ & 300 & N \\
\hline 4.75772 & 30 & 20 & 15 & $N$ & 200 & 150 & $N$ & 30 & $N$ & 300 & N \\
\hline 435773 & 30 & 15 & 15 & $N$ & 200 & 100 & $N$ & 30 & $N$ & 150 & N \\
\hline 435774 & 30 & 20 & 15 & $N$ & 200 & 100 & $N$ & 30 & $N$ & 200 & $N$ \\
\hline 435775 & 30 & 20 & 15 & $N$ & 200 & 100 & $N$ & 30 & $N$ & 200 & N \\
\hline 435776 & 20 & 15 & 10 & $N$ & 150 & 100 & $N$ & 30 & $N$ & 1,000 & $N$ \\
\hline 435777 & 30 & 15 & 20 & $N$ & 200 & 150 & $N$ & 50 & $N$ & 300 & N \\
\hline 435778 & 20 & 20 & 15 & $N$ & 100 & 70 & $N$ & 30 & $N$ & 300 & $N$ \\
\hline 435779 & 20 & 15 & 15 & $N$ & 150 & 70 & $N$ & 30 & $N$ & 200 & $N$ \\
\hline 435780 & 70 & 20 & 20 & $N$ & 200 & 150 & N & 50 & $N$ & 300 & $N$ \\
\hline 435781 & 30 & 50 & 30 & $N$ & 200 & 150 & $N$ & 50 & $N$ & 200 & N \\
\hline 435782 & 50 & 30 & 20 & 110 & 200 & 150 & $N$ & 50 & $N$ & 500 & N \\
\hline 435783 & 30 & 30 & 20 & $N$ & 150 & 150 & $N$ & 50 & $N$ & 700 & $N$ \\
\hline 435785 & 30 & 30 & 30 & $N$ & 200 & 150 & $N$ & 50 & $N$ & 300 & $N$ \\
\hline 435786 & 30 & 50 & 30 & $N$ & 300 & 150 & $N$ & 50 & $N$ & 200 & N \\
\hline 435787 & 30 & 50 & 15 & 30 & $\$ 100$ & 100 & $N$ & 150 & $N$ & 300 & $\$ 100$ \\
\hline 435788 & 50 & 50 & 20 & 10 & 200 & 150 & N & 70 & $N$ & 300 & $<100$ \\
\hline 435789 & 30 & 100 & 30 & 10 & 100 & 150 & $N$ & 150 & $N$ & 700 & 100 \\
\hline 435791 & 30 & 50 & 10 & 10 & 100 & 70 & $N$ & 70 & $N$ & 700 & $<100$ \\
\hline
\end{tabular}




\begin{tabular}{|c|c|c|c|c|c|c|c|c|c|}
\hline Sample & Latitude & Longitude & $\begin{array}{c}\text { Fe-pct. } \\
5\end{array}$ & $\begin{array}{c}\text { Mg-pet. } \\
s\end{array}$ & $\begin{array}{c}\text { Ca-pct. } \\
5\end{array}$ & $\begin{array}{c}\text { Ti-pct. } \\
5\end{array}$ & $\begin{array}{c}\text { Mn-ppi } \\
5\end{array}$ & $\begin{array}{c}\mathrm{Ag}-\mathrm{pp} \\
5\end{array}$ & $\begin{array}{c}\text { As-ppi } \\
s\end{array}$ \\
\hline 435792 & 653746 & $14651 \quad b$ & 5.00 & 1.50 & 1.5 & .700 & 1,000 & $N$ & $H$ \\
\hline 435793 & 653858 & 1465334 & 5.00 & 1.50 & .5 & .700 & 1,500 & $N$ & $N$ \\
\hline 435794 & 653858 & $14652 \quad 17$ & 5.00 & 1.50 & .7 & 1.000 & 1,500 & N & $H$ \\
\hline 435795 & 653930 & 1465136 & 5.00 & 1.50 & 1.0 & 1.000 & 1,500 & $N$ & $N$ \\
\hline 435797 & 653745 & 1465711 & 7.00 & 1.50 & 1.0 & 1.000 & 1,000 & .5 & $N$ \\
\hline 435798 & 653754 & $146 \quad 56 \quad 17$ & 3.00 & 1.50 & .7 & .700 & 1,000 & 6.5 & $N$ \\
\hline 435799 & $6536 \quad 15$ & 1465840 & 7.00 & 2.00 & 2.0 & .700 & 700 & 6.5 & $\mathrm{H}$ \\
\hline 435800 & $6536 \quad 6$ & 1465936 & 5.00 & 1.50 & 1.0 & .700 & 700 & $N$ & $N$ \\
\hline 435817 & 651440 & $146 \quad 21 \quad 47$ & 3.00 & 1.50 & 1.5 & .700 & 1,000 & $N$ & $N$ \\
\hline 435818 & 651422 & 1462116 & 7.00 & 1.50 & 1.0 & 1.000 & 500 & $N$ & $N$ \\
\hline 435819 & 651339 & 1462720 & 5.00 & 1.50 & .7 & 1.000 & 500 & N & $n$ \\
\hline 435820 & 651346 & 1462559 & 5.00 & 2.00 & 2.0 & 1.000 & 1,000 & $N$ & $N$ \\
\hline 435821 & 65175 & $\begin{array}{lll}146 & 28 & 32\end{array}$ & 5.00 & 1.50 & .5 & 1.000 & 500 & N & $H$ \\
\hline 435822 & 651641 & $14628 \quad 1$ & 7.00 & 2.00 & 2.0 & 1.000 & 2,000 & N & $N$ \\
\hline 435823 & 651717 & 1463244 & 7.00 & 1.50 & 2.0 & 1.000 & 1,000 & N & $N$ \\
\hline 435824 & 651658 & $14633 \quad 39$ & 10.00 & 2.00 & 3.0 & 1.000 & 2,000 & N & $N$ \\
\hline 435825 & 651357 & $14638 \quad 0$ & 7.00 & 1.50 & 3.0 & .700 & 2,000 & N & $N$ \\
\hline 435826 & 651345 & 146393 & 7.00 & 2.00 & 2.0 & 1.000 & 3,000 & N & $N$ \\
\hline 435827 & 65125 & 146400 & 7.00 & 1.50 & 3.0 & .700 & 700 & $N$ & $N$ \\
\hline 435828 & 651144 & 1464031 & 7.00 & 1.50 & 3.0 & 1.000 & 700 & N & $N$ \\
\hline 435829 & 65746 & 1463847 & 7.00 & 1.50 & 3.0 & .700 & 1,000 & $N$ & $N$ \\
\hline 435830 & 65750 & 1463935 & 5.00 & 1.50 & 2.0 & 1.000 & 700 & N & $N$ \\
\hline 435831 & $65 \quad 610$ & $146 \quad 40 \quad 44$ & 5.00 & 1.50 & 3.0 & 1.000 & 1,000 & $N$ & $N$ \\
\hline 435832 & 65540 & 1464023 & 7.00 & 1.50 & 2.0 & 1.000 & 1,000 & $N$ & $N$ \\
\hline 435835 & 6535 & 1462626 & 1.50 & 1.00 & .7 & .300 & 300 & $N$ & $N$ \\
\hline 435836 & 65351 & $146 \quad 26 \quad 2$ & 1.00 & .20 & .3 & .150 & 150 & N & $N$ \\
\hline 435837 & $\begin{array}{llll}b 5 & b & 24\end{array}$ & 1462559 & 5.00 & 1.50 & 3.0 & 1.000 & 1,000 & N & $N$ \\
\hline 435838 & 6565 & $146 \quad 2555$ & 5.00 & 1.50 & 2.0 & .700 & 700 & N & $N$ \\
\hline 435839 & $65 \quad 12 \quad 6$ & 1462741 & 7.00 & 2.00 & 3.0 & 1.000 & 1,500 & N & $N$ \\
\hline 435840 & 651149 & $146 \quad 2653$ & 10.00 & 2.00 & 3.0 & 1.000 & 3,000 & N & $N$ \\
\hline 435841 & $6512 \quad 9$ & $146 \quad 2437$ & 7.00 & 1.50 & 3.0 & 1.000 & 2,000 & $N$ & $N$ \\
\hline 435842 & 651223 & $146 \quad 2353$ & 10.00 & 2.00 & 3.0 & 1.000 & 3,000 & $N$ & $N$ \\
\hline 435843 & 651251 & 146212 & 5.00 & 1.50 & 1.5 & .700 & 2,000 & N & $N$ \\
\hline 435844 & 651223 & 1462030 & 7.00 & 1.50 & 2.0 & 1.000 & 1,500 & $N$ & $N$ \\
\hline 435845 & $65 \quad 1254$ & 1461641 & 10.00 & 2.00 & 3.0 & 1.000 & 2,000 & $N$ & $N$ \\
\hline 435846 & 651211 & $146 \quad 17 \quad 17$ & 7.00 & 1.50 & .5 & .700 & 2,000 & $N$ & $N$ \\
\hline 435847 & 651058 & 1461648 & 7.00 & 1.50 & 1.0 & 1.000 & 1,000 & N & $N$ \\
\hline 435848 & 651027 & 1461740 & 7.00 & 1.50 & .5 & .700 & 700 & $N$ & $N$ \\
\hline 435849 & 65813 & 146216 & 7.00 & 2.00 & 2.0 & .700 & 1,500 & $N$ & $H$ \\
\hline 435850 & 65740 & 1462053 & 7.00 & 2.00 & 2.0 & .700 & 1,000 & N & $N$ \\
\hline 435851 & $65 \quad 539$ & 1462049 & 7.00 & 2.00 & 3.0 & 1.000 & 1,500 & N & $H$ \\
\hline 435852 & 6558 & 1462030 & 7.00 & 2.00 & 5.0 & 1.000 & 2,000 & $H$ & $N$ \\
\hline 435853 & 6523 & $146 \quad 2230$ & 10.00 & 2.00 & 2.0 & .700 & 2,000 & N & $n$ \\
\hline 435854 & 65137 & $14622 \quad 23$ & 10.00 & 2.00 & 3.0 & 1.000 & 1,500 & N & $\mathrm{H}$ \\
\hline 435855 & 65150 & $146 \quad 15 \quad 11$ & 7.00 & 2.00 & 3.0 & 1.000 & 1,500 & N & $N$ \\
\hline
\end{tabular}


TAELE 2.--RESULTS OF SPECTROGRAPHIC ANALYSES OF NURE STREAM-SEDIMENT SAMPLES FROM THE LIVENGOOD

AND WESTERN $1 / 3$ OF THE CIRCLE QUADRANGLES, ALASKA--ContinUEd

\begin{tabular}{|c|c|c|c|c|c|c|c|c|c|c|}
\hline Satiple & $\begin{array}{c}\text { B-ppi } \\
5\end{array}$ & $\begin{array}{c}\text { Ba-ppofi } \\
\vdots\end{array}$ & $\begin{array}{c}\text { Be-ppi } \\
5\end{array}$ & 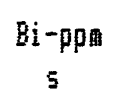 & $\begin{array}{c}\text { Co-ppi } \\
5\end{array}$ & $\begin{array}{c}C r-p p \text { if } \\
s\end{array}$ & $\begin{array}{c}\text { Cu-ppo } \\
s\end{array}$ & $\begin{array}{c}\text { La-ppil } \\
5\end{array}$ & $\begin{array}{c}\text { Mo-ppon } \\
5\end{array}$ & $\begin{array}{c}N b-p p w \\
5\end{array}$ \\
\hline 435792 & 100 & 1,000 & 5.0 & $<10$ & 15 & 100 & 20 & 200 & $<5$ & 20 \\
\hline 435793 & 100 & 1,500 & 3.0 & N & 20 & 100 & 20 & 500 & $<5$ & 20 \\
\hline 435794 & 150 & 2,000 & 1.5 & N & 20 & 100 & 30 & 50 & $N$ & $<20$ \\
\hline 435795 & 100 & 1,500 & 2.0 & N & 20 & 100 & 20 & 50 & N & $<20$ \\
\hline 435797 & 100 & 1,500 & 1.5 & $N$ & 20 & 150 & 30 & 70 & N & $<20$ \\
\hline 435798 & 100 & 1,500 & 1.5 & $N$ & 15 & 100 & 30 & 70 & $N$ & $<20$ \\
\hline 435799 & 100 & 1,500 & 1.5 & $N$ & 15 & 150 & 50 & 50 & N & $<20$ \\
\hline 435800 & 100 & 1,000 & 2.0 & $N$ & 15 & 100 & 15 & 50 & $\langle 5$ & 20 \\
\hline 435817 & 100 & 1,000 & 1.0 & $N$ & 15 & 200 & 15 & 50 & $N$ & $<20$ \\
\hline 435818 & 100 & 1,000 & 1.0 & N & 10 & 200 & 15 & 70 & $<5$ & 120 \\
\hline 435819 & 100 & 1,000 & 1.0 & $N$ & 10 & 200 & 20 & 70 & $N$ & $<20$ \\
\hline 435820 & 100 & 1,500 & 1.0 & $N$ & 20 & 200 & 30 & 70 & N & 20 \\
\hline 435821 & 70 & 500 & $\langle 1.0$ & $N$ & 15 & 150 & 30 & 50 & N & $N$ \\
\hline 435822 & 70 & 1,000 & 41.0 & $n$ & 20 & 200 & 50 & 50 & $H$ & 20 \\
\hline 435823 & 100 & 700 & $\langle 1.0$ & $N$ & 15 & 200 & 50 & 50 & N & $<20$ \\
\hline 435824 & 100 & 1,000 & $\langle 1.0$ & $N$ & 20 & 150 & 50 & 50 & $N$ & $<20$ \\
\hline 435825 & 100 & 1,500 & $\langle 1.0$ & $N$ & 15 & 200 & 15 & 70 & $N$ & $<20$ \\
\hline $43582 b$ & 100 & 500 & 1.0 & $N$ & 30 & 200 & 30 & 70 & $\mathrm{~N}$ & $<20$ \\
\hline 435827 & 70 & 1,000 & $\langle 1.0$ & $N$ & 15 & 200 & 15 & 50 & $N$ & $<20$ \\
\hline 435828 & 100 & 1,000 & $\langle 1.0$ & $N$ & 15 & 200 & 20 & 50 & N & $<20$ \\
\hline 435829 & 100 & 1,500 & $\langle 1.0$ & $N$ & 15 & 100 & 10 & 50 & $N$ & $<20$ \\
\hline 435830 & 70 & 700 & 1.0 & N & 15 & 150 & 15 & 50 & N & $<20$ \\
\hline 435831 & 100 & 1,000 & $\langle 1.0$ & $N$ & 15 & 200 & 10 & 50 & $N$ & $<20$ \\
\hline 435832 & 70 & 1,500 & $\$ 1.0$ & $N$ & 20 & 200 & 20 & 50 & $N$ & $<20$ \\
\hline 4.35835 & 20 & 700 & 1.0 & $N$ & 7 & 70 & 15 & 50 & N & $\mathrm{N}$ \\
\hline 435836 & $<10$ & 200 & 1.0 & $N$ & $<5$ & 30 & 15 & 30 & N & $N$ \\
\hline 435837 & 100 & 1,500 & 1.5 & H & 15 & 200 & 15 & 50 & $<5$ & 20 \\
\hline 435838 & 70 & 1,000 & 1.0 & N & 15 & 150 & 20 & 50 & $N$ & $<20$ \\
\hline 435839 & 70 & 1,000 & $\langle 1.0$ & H & 20 & 200 & 15 & 50 & N & $<20$ \\
\hline 435840 & 50 & 2,000 & 1.0 & N & 20 & 200 & 15 & 70 & N & $<20$ \\
\hline 435841 & 100 & 1,500 & 1.0 & N & 15 & 200 & 20 & 50 & $N$ & $<20$ \\
\hline 435842 & 200 & 1,500 & $\langle 1.0$ & $N$ & 20 & 200 & 30 & 70 & N & $<20$ \\
\hline 435843 & 50 & 1,500 & $\langle 1.0$ & N & 15 & 150 & 15 & 50 & $\mathrm{~N}$ & $N$ \\
\hline 435844 & 100 & 1,500 & $\langle 1.0$ & $H$ & 15 & 200 & 15 & 50 & $N$ & $<20$ \\
\hline 435845 & 70 & 1,500 & 1.0 & N & 20 & 200 & 20 & 70 & $N$ & $<20$ \\
\hline 435846 & 100 & 1,000 & $\langle 1.0$ & $N$ & 10 & 200 & 15 & 30 & $<5$ & $N$ \\
\hline 435847 & 100 & 1,500 & $\langle 1.0$ & N & 10 & 150 & 10 & 50 & $N$ & $<20$ \\
\hline 435848 & 70 & 1,000 & $\langle 1.0$ & N & 15 & 200 & 15 & 70 & $N$ & $<20$ \\
\hline 435849 & 150 & 2,000 & 1.0 & N & 15 & 200 & 30 & 70 & $<5$ & $<20$ \\
\hline 435850 & 100 & 1,500 & $\langle 1.0$ & H & 15 & 200 & 30 & 50 & $N$ & $<20$ \\
\hline 435851 & 70 & 1,500 & 41.0 & $N$ & 15 & 150 & 15 & 50 & $N$ & $<20$ \\
\hline 435852 & 70 & 1,000 & $\langle 1.0$ & N & 15 & 200 & 10 & 50 & $\mathrm{H}$ & $<20$ \\
\hline 435853 & 100 & 2,000 & 1.0 & N & 15 & 200 & 30 & 50 & $\langle 5$ & $<20$ \\
\hline 435854 & 100 & 2,000 & 1.0 & H & 15 & 200 & 15 & 50 & N & $<20$ \\
\hline 435855 & 70 & 2,000 & 1.0 & N & 20 & 300 & 30 & 70 & $N$ & $<20$ \\
\hline
\end{tabular}


TABLE 2.--RESUL TS OF SPECTROGRAPHIC AMALYSES OF NURE STREAM-SEDIMENT SAMPLES FROH THE LIVENGOOD

AND WESTERN $1 / 3$ OF THE CIRCLE QUADRANGLES, ALASKA--Continued

\begin{tabular}{|c|c|c|c|c|c|c|c|c|c|c|c|}
\hline Sample & $\begin{array}{l}\mathrm{Ni}-\mathrm{pp} \text { i } \\
\mathrm{s}\end{array}$ & $\begin{array}{c}\mathrm{Pb}-\mathrm{ppl} \\
5\end{array}$ & $\begin{array}{c}\text { Sc-ppi } \\
5\end{array}$ & $\begin{array}{c}\text { Sn-ppi } \\
5\end{array}$ & $\begin{array}{c}\mathrm{Sr}-\mathrm{ppi} \\
5\end{array}$ & $\begin{array}{c}\text { V-ppon } \\
5\end{array}$ & $\begin{array}{c}\text { H-pp亩 } \\
5\end{array}$ & $\begin{array}{c}Y \text {-ppmit } \\
5\end{array}$ & $\begin{array}{c}2 n-p p \text { i } \\
5\end{array}$ & $\begin{array}{c}2 r-p p=1 \\
5\end{array}$ & $\begin{array}{c}\text { Th-pplit } \\
5\end{array}$ \\
\hline 435792 & 30 & 50 & 30 & 50 & 150 & 100 & $N$ & 150 & N & 700 & $<100$ \\
\hline 4.55793 & 30 & 100 & 30 & 20 & 100 & 100 & $N$ & 150 & N & 1,000 & 200 \\
\hline 435794 & 30 & 50 & 30 & $N$ & 150 & 150 & $N$ & 50 & $N$ & 300 & $N$ \\
\hline 435795 & 50 & 50 & 20 & $N$ & 150 & 150 & $N$ & 50 & $N$ & 200 & $N$ \\
\hline 435797 & 50 & 70 & 30 & $\$ 10$ & 200 & 150 & $N$ & 50 & $N$ & 500 & $N$ \\
\hline 435798 & 30 & 100 & 20 & 10 & 200 & 150 & $N$ & 50 & N & 300 & $n$ \\
\hline 435799 & 30 & 50 & 20 & 15 & 200 & 150 & N & 50 & N & 200 & $\mathrm{~N}$ \\
\hline 435800 & 50 & 20 & 10 & 10 & 200 & 100 & $N$ & 50 & $N$ & 500 & N \\
\hline 435817 & 30 & 20 & 10 & $<10$ & 200 & 100 & H & 30 & $N$ & 200 & $N$ \\
\hline 435818 & 30 & 30 & 20 & $N$ & 200 & 150 & $N$ & 50 & N & 500 & $N$ \\
\hline 435819 & 20 & 30 & 20 & $N$ & 150 & 150 & $N$ & 50 & $N$ & 500 & $N$ \\
\hline 435820 & 30 & 30 & 20 & $N$ & 200 & 150 & $N$ & 50 & $<200$ & 500 & $N$ \\
\hline 435821 & 30 & 20 & 15 & $N$ & 100 & 100 & $N$ & 30 & N & 300 & $N$ \\
\hline 435822 & 30 & 20 & 20 & $N$ & 150 & 200 & N & 30 & $N$ & 200 & $N$ \\
\hline 435923 & 30 & 20 & 20 & $N$ & 150 & 100 & $N$ & 30 & $N$ & 500 & $N$ \\
\hline 435824 & 30 & 20 & 30 & $N$ & 150 & 150 & $N$ & 70 & N & 300 & $N$ \\
\hline 435925 & 30 & 20 & 20 & $\mathrm{H}$ & 200 & 100 & N & 50 & $N$ & 300 & $N$ \\
\hline 435826 & 50 & 30 & 20 & $N$ & 200 & 200 & N & 50 & N & 300 & $N$ \\
\hline 435827 & 30 & 20 & 15 & H & 300 & 150 & $N$ & 30 & $N$ & 500 & $N$ \\
\hline 435828 & 30 & 20 & 20 & $N$ & 300 & 150 & N & 50 & N & 500 & $N$ \\
\hline 435829 & 20 & 30 & 20 & N & 300 & 150 & $N$ & 30 & $N$ & 700 & $N$ \\
\hline 435830 & 30 & 20 & 15 & $N$ & 200 & 100 & N & 20 & $N$ & 500 & $\mathrm{~N}$ \\
\hline 435831 & 30 & 20 & 20 & $N$ & 300 & 100 & $\mathrm{~N}$ & 50 & $N$ & 700 & $N$ \\
\hline 435832 & 30 & 30 & 20 & $N$ & 300 & 150 & $N$ & 50 & $N$ & 500 & $N$ \\
\hline 435835 & 20 & 15 & 15 & N & $\langle 100$ & 100 & N & 30 & N & 100 & $N$ \\
\hline 435836 & 20 & $\$ 10$ & 7 & $N$ & $N$ & 30 & N & 20 & $N$ & 50 & $N$ \\
\hline 435837 & 30 & 30 & 20 & $N$ & 300 & 150 & $N$ & 70 & $N$ & 500 & $N$ \\
\hline 435838 & 30 & 30 & 20 & $N$ & 200 & 100 & N & 30 & $N$ & 300 & $N$ \\
\hline 435839 & 30 & 20 & 20 & N & 200 & 100 & N & 30 & N & 500 & N \\
\hline 435840 & 30 & 50 & 20 & $N$ & 300 & 200 & $N$ & 30 & $N$ & 300 & $N$ \\
\hline 435941 & 30 & 20 & 20 & $\mathrm{~N}$ & 300 & 150 & $N$ & 50 & N & 700 & $H$ \\
\hline 435842 & 50 & 50 & 20 & N & 500 & 150 & N & 50 & $N$ & 300 & $N$ \\
\hline 435843 & 30 & 30 & 15 & $N$ & 150 & 150 & $N$ & 30 & N & 700 & $N$ \\
\hline 435844 & 30 & 20 & 20 & N & 200 & 150 & $N$ & 30 & $N$ & 500 & N \\
\hline 435845 & 50 & 30 & 20 & $N$ & 300 & 150 & $N$ & 50 & N & 500 & $N$ \\
\hline 435846 & 30 & 20 & 15 & $N$ & 100 & 100 & $N$ & 30 & $N$ & 31,000 & $N$ \\
\hline 435847 & 20 & 30 & 15 & $N$ & 200 & 150 & $N$ & 30 & $\mathrm{~N}$ & 1,000 & $N$ \\
\hline 435848 & 30 & 20 & 15 & N & 100 & 100 & N & 20 & $N$ & 300 & $N$ \\
\hline 435849 & 50 & 30 & 20 & $\mathrm{~N}$ & 300 & 150 & $N$ & 50 & N & 500 & $N$ \\
\hline 435850 & 30 & 30 & 15 & $N$ & 200 & 150 & $N$ & 30 & N & 500 & $N$ \\
\hline 435851 & 20 & 20 & 15 & $N$ & 200 & 150 & $N$ & 30 & $N$ & 700 & $N$ \\
\hline 435852 & 20 & 20 & 20 & N & 300 & 100 & $N$ & 30 & $N$ & 700 & $N$ \\
\hline 435853 & 30 & 50 & 20 & $\mathrm{~N}$ & 200 & 150 & $N$ & 30 & $N$ & 300 & $N$ \\
\hline 435854 & 30 & 30 & 20 & $N$ & 300 & 150 & $\mathrm{~N}$ & 50 & N & 700 & H \\
\hline 435855 & 30 & 30 & 20 & N & 300 & 150 & N & 50 & N & 500 & $N$ \\
\hline
\end{tabular}


TAELE 2.--RESULTS OF SPECTROGRAPHIC AMALYSES OF NURE STREAM-SEDIMENT SAMPLES FROM THE LIVENGDOD

AND WESTERN $1 / 3$ OF THE CIRCLE QUADRANGLES, ALASKA-ContinUed

\begin{tabular}{|c|c|c|c|c|c|c|c|c|c|}
\hline Sample & Latitude & Longitude & $\begin{array}{c}\text { Fe-pct. } \\
\Xi\end{array}$ & $\begin{array}{c}\mathrm{Mg}_{\mathrm{g}}-\mathrm{pct} . \\
\mathrm{s}\end{array}$ & $\begin{array}{c}\text { ca-pct. } \\
5\end{array}$ & $\begin{array}{c}\text { Ti-pct. } \\
5\end{array}$ & $\begin{array}{c}\text { Mn-ppi } \\
5\end{array}$ & $\begin{array}{c}A q-p p \operatorname{li} \\
5\end{array}$ & $\begin{array}{c}\text { As-pp } \\
5\end{array}$ \\
\hline 435856 & $65 \quad 132$ & $146 \quad 14 \quad 18$ & 7.00 & 2.00 & 3.0 & 1.000 & 1,500 & $N$ & N \\
\hline 435857 & 653611 & 146568 & 7.00 & 2.00 & .5 & .700 & 1,000 & 6.5 & $N$ \\
\hline 435858 & $6535 \quad 17$ & 1465725 & 7.00 & 1.50 & 1.5 & .700 & 1,500 & $<.5$ & $N$ \\
\hline 435859 & 653522 & 1465613 & 7.00 & 1.50 & .7 & .500 & 1,000 & 1.0 & $N$ \\
\hline 435861 & 65336 & 1465733 & 7.00 & 1.50 & 2.0 & .700 & 2,000 & $N$ & $N$ \\
\hline 435862 & 653146 & 1465625 & 5.00 & 1.50 & .7 & .700 & 500 & $N$ & N \\
\hline 435864 & 652746 & 1465715 & 5.00 & 1.50 & .7 & .700 & 700 & N & $N$ \\
\hline 435867 & 65253 & $14658 \quad 5$ & 10.00 & 2.00 & 2.0 & 1.000 & 1,000 & $N$ & $N$ \\
\hline 435968 & 652527 & 1465815 & 7.00 & 1.50 & .5 & 1.000 & 1,000 & $H$ & $N$ \\
\hline 435869 & 652249 & 146592 & 7.00 & 1.50 & 3.0 & 1.000 & 700 & $N$ & $N$ \\
\hline 435870 & 65200 & $14659 \quad 16$ & 7.00 & 1.50 & 3.0 & 1.000 & 700 & N & N \\
\hline 435871 & 652016 & $14658 \quad 8$ & 5.00 & 1.50 & 2.0 & 1.000 & 1,000 & N & N \\
\hline 435872 & $6519 \quad 17$ & 1465711 & 7.00 & 1.50 & 3.0 & 1.000 & 700 & N & N \\
\hline 435873 & 651832 & 1465724 & 10.00 & 2.00 & 3.0 & 1.000 & 2,000 & $N$ & N \\
\hline 435874 & $6518 \quad 6$ & 1465732 & 7.00 & 1.50 & 3.0 & 1.000 & 1,000 & $N$ & N \\
\hline 435875 & 651653 & 1465533 & 5.00 & 1.50 & 2.0 & $>1.000$ & 700 & $N$ & N \\
\hline 435876 & 651632 & 146564 & 5.00 & 1.50 & 2.0 & 1.000 & 500 & $N$ & $N$ \\
\hline 435877 & 651449 & 1465736 & 10.00 & 3.00 & 5.0 & 31.000 & 2,000 & H & N \\
\hline 435878 & $65 \quad 15 \quad 2$ & 1465630 & 10.00 & 2.00 & 3.0 & 31.000 & 1,000 & N & N \\
\hline 435979 & 651344 & $14659 \quad 44$ & 10.00 & 2.00 & 3.0 & 1.000 & 1,000 & N & $N$ \\
\hline 435890 & 651148 & 1465715 & 10.00 & 2.00 & 3.0 & 1.000 & 2,000 & N & N \\
\hline 435881 & 651123 & 1465645 & 10.00 & 1.50 & 2.0 & .700 & 2,000 & $N$ & N \\
\hline 435882 & 651117 & 1465820 & 10.00 & 1.50 & 3.0 & .700 & 500 & $H$ & $N$ \\
\hline 435983 & 651023 & $14659 \quad 12$ & 7.00 & 1.50 & 3.0 & 1.000 & 1,000 & N & N \\
\hline 435884 & 651111 & 1465433 & 7.00 & 1.50 & 3.0 & 1.000 & 1,000 & $N$ & N \\
\hline 435914 & 651317 & $14646 \quad 27$ & 7.00 & 1.50 & 2.0 & 71.000 & 1,000 & $N$ & $\mathrm{~N}$ \\
\hline 435915 & 651350 & $146 \quad 46 \quad 44$ & 7.00 & 1.50 & 1.5 & 1.000 & 3,000 & N & N \\
\hline 435916 & 65139 & 1464655 & 7.00 & 1.50 & 2.0 & 1.000 & 2,000 & N & $N$ \\
\hline 435917 & 651649 & 1463847 & 10.00 & 2.00 & 2.0 & 1.000 & 1,000 & N & $N$ \\
\hline 435918 & 651623 & 1463827 & 10.00 & 1.50 & 3.0 & 1.000 & 1,000 & N & $N$ \\
\hline 435919 & 651551 & 1464138 & 7.00 & 1.50 & .7 & 1.000 & 1,000 & N & N \\
\hline 435920 & 651524 & $146 \quad 4350$ & 7.00 & 1.50 & 5.0 & 1.000 & 1,000 & $N$ & N \\
\hline 435921 & 651532 & 1464435 & 10.00 & 2.00 & 2.0 & 1.000 & 1,000 & N & $N$ \\
\hline 435922 & 651620 & 1464353 & 10.00 & 2.00 & 3.0 & 1.000 & 1,000 & $N$ & N \\
\hline 435923 & 651950 & 1464627 & 5.00 & 1.50 & .5 & 1.000 & 700 & N & N \\
\hline 435924 & 652053 & $14647 \quad 11$ & 5.00 & 1.50 & 1.5 & 1.000 & 500 & N & N \\
\hline 435925 & 652054 & $14648 \quad 15$ & 5.00 & 1.50 & 1.5 & 1.000 & 500 & $N$ & N \\
\hline 435926 & 652324 & $146 \quad 46 \quad 32$ & 7.00 & 1.50 & .7 & 1.000 & 1,000 & N & N \\
\hline 435927 & 652343 & 1464550 & 5.00 & 1.50 & 1.5 & 1.000 & 1,500 & N & N \\
\hline 435928 & 65250 & $14648 \quad 15$ & 5.00 & 1.50 & 1.0 & 1.000 & 700 & N & N \\
\hline 435929 & 652442 & 1464843 & 5.00 & 1.50 & 1.0 & 1.000 & 1,000 & $N$ & N \\
\hline 435930 & 652554 & $14647 \quad 4$ & 5.00 & 1.50 & 1.5 & 1.000 & 1,500 & 1.0 & N \\
\hline 435931 & 652611 & 1464546 & 5.00 & 1.50 & 2.0 & 1.000 & 1,000 & $N$ & $N$ \\
\hline 435932 & 652948 & 1464821 & 5.00 & 1.50 & .5 & 1.000 & 700 & N & N \\
\hline 435933 & 65299 & 1464838 & 7.00 & 2.00 & 1.5 & 1.000 & 1,000 & N & $N$ \\
\hline
\end{tabular}


TABLE 2. - RESULTS OF SPECTROGRAFHIC ANALYSES OF NURE STREAM-SEDIMENT SAMPLES FRDM THE LIVENGOOD

AND WESTERN $1 / 3$ OF THE CIRCLE QUADRANGLES, ALASKA--ContinuEd

\begin{tabular}{|c|c|c|c|c|c|c|c|c|c|c|}
\hline Sample & $\begin{array}{c}\text { B-ppi } \\
5\end{array}$ & $\begin{array}{c}\text { Ba-ppl } \\
5\end{array}$ & $\begin{array}{c}\text { Be-ppi } \\
5\end{array}$ & $\begin{array}{c}B i-p p A \\
5\end{array}$ & $\begin{array}{c}\text { [0-ppi } \\
s\end{array}$ & $\begin{array}{c}\text { Cr-ppa } \\
5\end{array}$ & $\begin{array}{c}\text { Cu-ppm } \\
5\end{array}$ & $\begin{array}{c}\text { La-ppm } \\
5\end{array}$ & $\begin{array}{c}\text { Mo-ppo } \\
s\end{array}$ & 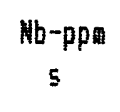 \\
\hline 435956 & 100 & 1,500 & 1.0 & $N$ & 15 & 150 & 20 & 50 & $N$ & $\langle 20$ \\
\hline 435957 & 100 & 2,000 & 1.0 & N & 20 & 300 & 50 & 70 & 5 & $\langle 20$ \\
\hline 435858 & 100 & 1,500 & 3.0 & $\mathrm{~N}$ & 20 & 150 & 50 & 70 & $\mathrm{~N}$ & $\langle 20$ \\
\hline 435859 & 100 & 1,000 & 15.0 & 20 & 15 & 150 & 70 & 70 & 10 & $\langle 20$ \\
\hline 4355861 & 100 & 1,000 & $\langle 1.0$ & N & 20 & 200 & 20 & 70 & $\langle 5$ & 20 \\
\hline 435862 & 70 & 1,000 & $\{1.0$ & $N$ & 20 & 300 & 15 & 50 & $N$ & 320 \\
\hline 435864 & 50 & 700 & $\langle 1.0$ & N & 15 & 150 & 10 & 50 & $N$ & N \\
\hline 435867 & 100 & 1,500 & 1.0 & N & 10 & 200 & 30 & 50 & $N$ & N \\
\hline 435968 & 70 & 500 & 1.0 & $\mathrm{~N}$ & 15 & 150 & 30 & 50 & $\mathrm{~N}$ & $\mathrm{~N}$ \\
\hline 435869 & 70 & 1,000 & $\{1.0$ & $N$ & 15 & 150 & 20 & 50 & $N$ & $\ll 20$ \\
\hline 435870 & 70 & 1,000 & 1.0 & $N$ & 15 & 200 & 15 & 50 & $N$ & 20 \\
\hline 435871 & 100 & 700 & $\langle 1.0$ & N & 15 & 150 & 10 & 50 & $N$ & $\langle 20$ \\
\hline 435872 & 70 & 1,000 & 1.0 & $N$ & 15 & 200 & 15 & 30 & N & $\langle 20$ \\
\hline 435873 & 70 & 1,500 & $\langle 1.0$ & $N$ & 20 & 300 & 20 & 70 & $N$ & $<20$ \\
\hline 435874 & 70 & 1,500 & $\{1.0$ & $N$ & 15 & 200 & 15 & 50 & $N$ & $\mathrm{~N}$ \\
\hline 435875 & 100 & 1,000 & 1.0 & $N$ & 10 & 150 & 15 & 50 & $N$ & $<20$ \\
\hline 435876 & 70 & 1,000 & $\{1.0$ & $N$ & 10 & 150 & 10 & 30 & $N$ & 20 \\
\hline 435877 & 70 & 700 & $\mathrm{~N}$ & $\mathrm{H}$ & 15 & 200 & 30 & $\mathrm{~N}$ & N & $\langle 20$ \\
\hline 435878 & 100 & 500 & $\langle 1.0$ & N & 20 & 200 & 50 & N & $N$ & $\langle 20$ \\
\hline 435879 & 100 & 1,500 & $\langle 1.0$ & $N$ & 20 & 200 & 30 & 70 & $N$ & $N$ \\
\hline 435880 & 70 & 1,000 & $\ll 1.0$ & $\mathrm{~N}$ & 30 & 200 & 30 & 70 & $N$ & $\langle 20$ \\
\hline 435881 & 70 & 700 & $\langle 1.0$ & N & 20 & 200 & 15 & 30 & $N$ & $\mathrm{~N}$ \\
\hline 435862 & 70 & 1,000 & 1.0 & $N$ & 15 & 300 & 30 & 50 & $N$ & $\mathrm{~N}$ \\
\hline 435883 & 70 & 1,000 & 1.0 & N & 15 & 200 & 20 & 50 & $N$ & N \\
\hline 435584 & 70 & 1,000 & 1.0 & $N$ & 15 & 200 & 20 & 50 & $N$ & N \\
\hline 4359914 & 150 & 1,000 & 1.0 & $N$ & 20 & 200 & 15 & 70 & $N$ & 20 \\
\hline 435915 & 100 & 1,000 & 1.0 & $\mathrm{~N}$ & 30 & 200 & 20 & 70 & $\mathrm{~N}$ & 20 \\
\hline 435916 & 150 & 1,500 & 1.0 & N & 20 & 200 & 20 & 70 & $N$ & $<20$ \\
\hline 4355917 & 100 & 1,000 & 1.0 & N & 20 & 200 & 50 & 50 & $N$ & $\langle 20$ \\
\hline 435918 & 100 & 700 & 1.0 & N & 20 & 150 & 30 & 50 & $N$ & $<20$ \\
\hline 435919 & 100 & 1,000 & 1.0 & $N$ & 20 & 150 & 20 & 50 & $N$ & $\ll 20$ \\
\hline 435920 & 100 & 700 & 1.0 & N & 20 & 100 & 30 & 30 & $N$ & 20 \\
\hline 4355921 & 100 & 1,000 & 1.0 & N & 20 & 150 & 50 & 50 & N & $<20$ \\
\hline 435922 & 100 & 700 & $\langle 1.0$ & $N$ & 20 & 100 & 30 & 30 & $N$ & $\langle 20$ \\
\hline 4355923 & 150 & 700 & 1.0 & $N$ & 15 & 50 & 15 & 30 & $N$ & 20 \\
\hline 435924 & 100 & 1,000 & 1.0 & $N$ & 15 & 100 & 15 & 50 & $N$ & $\langle 20$ \\
\hline 4355925 & 100 & 1,000 & 1.0 & $\mathrm{~N}$ & 15 & 100 & 15 & 70 & $N$ & $\langle 20$ \\
\hline 435926 & 150 & 700 & 1.5 & $N$ & 20 & 70 & 20 & 70 & H & $\langle 20$ \\
\hline 435927 & 150 & 1,000 & 1.5 & $\mathrm{~N}$ & 15 & 100 & 15 & 70 & $N$ & $\langle 20$ \\
\hline 435928 & 150 & 700 & 1.5 & N & 15 & 70 & 15 & 70 & $N$ & 20 \\
\hline 4355929 & 150 & 700 & 1.0 & $N$ & 15 & 70 & 20 & 70 & $N$ & $<20$ \\
\hline 435930 & 100 & 1,000 & 1.0 & N & 20 & 100 & 20 & 70 & $N$ & 20 \\
\hline 435931 & 100 & 1,000 & 1.0 & N & 20 & 150 & 20 & 70 & N & 20 \\
\hline 435932 & 100 & 700 & 1.0 & N & 15 & 100 & 20 & 50 & $N$ & 20 \\
\hline 435933 & 100 & 1,000 & 1.0 & $N$ & 20 & 200 & 30 & 70 & 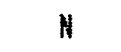 & 20 \\
\hline
\end{tabular}


TABLE 2.--RESULTS OF SPECTROGRAPHIC AMALYSES OF NURE STREAM-SEDIMENT SAMPLES FROM THE LIVENGODD

AND WESTEFN $1 / 3$ OF THE CIRCLE QUADRANGLES, ALASKA--Continued

\begin{tabular}{|c|c|c|c|c|c|c|c|c|c|c|c|}
\hline Sample & $\begin{array}{c}\text { Ni-ppi } \\
s\end{array}$ & $\begin{array}{c}\mathrm{Pb}-\mathrm{pp} \\
\mathrm{s}\end{array}$ & $\begin{array}{c}\text { St-ppi } \\
5\end{array}$ & $\begin{array}{c}5 n-p p \\
s\end{array}$ & $\begin{array}{c}5 r-p p i \\
5\end{array}$ & $\begin{array}{c}\text { V-ppon } \\
5\end{array}$ & $\begin{array}{c}W-p p m \\
5\end{array}$ & $\underset{3}{Y-p p \text { n }}$ & $\begin{array}{c}2 n-p p \text { n } \\
5\end{array}$ & $\begin{array}{c}7 r-p p \text { i } \\
s\end{array}$ & $\begin{array}{c}\text { Th-pp) } \\
5\end{array}$ \\
\hline 435856 & 30 & 20 & 20 & $\mathrm{~N}$ & 300 & 100 & $N$ & 30 & $N$ & 500 & $N$ \\
\hline 435857 & 70 & 100 & 30 & $<10$ & 200 & 150 & $\mathrm{~N}$ & 50 & 200 & 300 & $N$ \\
\hline 435858 & 50 & 70 & 20 & $<10$ & 200 & 100 & $\mathrm{~N}$ & 100 & 200 & 200 & $N$ \\
\hline 435859 & 50 & 100 & 20 & 15 & 100 & 100 & $N$ & 70 & 300 & 300 & $N$ \\
\hline 435861 & 50 & 50 & 20 & $N$ & 200 & 100 & $N$ & 30 & $<200$ & 300 & $N$ \\
\hline 435862 & 50 & 20 & 20 & $N$ & 150 & 100 & N & 30 & $N$ & 200 & $N$ \\
\hline 435864 & 20 & 20 & 15 & $N$ & 100 & 100 & $N$ & 20 & $N$ & 300 & $N$ \\
\hline 435967 & 50 & 30 & 20 & $N$ & 200 & 150 & $N$ & 50 & $N$ & 500 & $N$ \\
\hline 455968 & 30 & 20 & 20 & $\mathrm{~N}$ & 100 & 150 & $N$ & 30 & $\mathrm{~N}$ & 500 & $\mathrm{~N}$ \\
\hline 435869 & 30 & 20 & 20 & $N$ & 200 & 150 & $\ddot{N}$ & 30 & N & 500 & N \\
\hline 435970 & 30 & 20 & 20 & N & 200 & 150 & N & 30 & $N$ & 300 & $N$ \\
\hline 435971 & 30 & 15 & 15 & $\mathrm{~N}$ & 150 & 100 & $\ddot{N}$ & 30 & N & 300 & N \\
\hline 435872 & 30 & 20 & 15 & $N$ & 200 & 150 & N & 50 & N & 700 & $N$ \\
\hline 4.35873 & 50 & 30 & 20 & $N$ & 200 & 200 & $N$ & 50 & $N$ & 700 & $N$ \\
\hline 435874 & 30 & 20 & 20 & $N$ & 200 & 150 & N & 50 & $N$ & 500 & $N$ \\
\hline 435875 & 30 & 20 & 20 & $N$ & 200 & 100 & $N$ & 50 & $N$ & 1,000 & $N$ \\
\hline 435876 & 30 & 20 & 15 & $N$ & 200 & 100 & N & 30 & $N$ & 300 & $N$ \\
\hline 435877 & 30 & 20 & 30 & $N$ & $<100$ & 150 & $N$ & 20 & $N$ & 500 & $N$ \\
\hline 435878 & 50 & 20 & 30 & $N$ & 100 & 100 & N & 50 & N & 1,000 & $N$ \\
\hline 435979 & 50 & 30 & 30 & $N$ & 200 & 100 & $N$ & 50 & $N$ & 700 & $N$ \\
\hline 435880 & 70 & 20 & 30 & $N$ & 150 & 150 & $N$ & 50 & $N$ & 300 & N \\
\hline 435881 & 30 & 20 & 15 & $N$ & 150 & 100 & $N$ & 20 & $N$ & 300 & $N$ \\
\hline 435882 & 30 & 30 & 30 & $N$ & 200 & 150 & N & 50 & $N$ & 200 & $N$ \\
\hline 435883 & 30 & 20 & 20 & $N$ & 300 & 150 & $N$ & 30 & N & 500 & N \\
\hline 435884 & 50 & 20 & 20 & $N$ & 200 & 150 & $N$ & 50 & N & 700 & $N$ \\
\hline 435914 & 50 & 30 & 20 & $N$ & 200 & 150 & $N$ & 50 & $N$ & 700 & $N$ \\
\hline 435915 & 50 & 30 & 20 & 110 & 200 & 100 & N & 50 & $N$ & 200 & $N$ \\
\hline 435916 & 50 & 30 & 20 & $N$ & 200 & 150 & $N$ & 50 & $N$ & 700 & $N$ \\
\hline 435917 & 50 & 30 & 20 & $N$ & 200 & 150 & $N$ & 50 & $N$ & 300 & $N$ \\
\hline 435918 & 30 & 20 & 20 & $N$ & 150 & 150 & $N$ & 50 & $N$ & 300 & $N$ \\
\hline 435919 & 50 & 20 & 20 & $N$ & 200 & 150 & $N$ & 30 & $N$ & 500 & $N$ \\
\hline 435920 & 30 & 20 & 20 & $N$ & 150 & 150 & $N$ & 30 & $N$ & 300 & $N$ \\
\hline 435921 & 50 & 30 & 20 & $N$ & 150 & 200 & $N$ & 30 & $N$ & 300 & $\mathbf{N}$ \\
\hline 435922 & 30 & 20 & 20 & $N$ & 150 & 200 & $N$ & 50 & $N$ & 500 & $N$ \\
\hline 435923 & 20 & 20 & 7 & $N$ & 150 & 70 & $N$ & 20 & $N$ & 300 & $N$ \\
\hline 435924 & 30 & 30 & 15 & $N$ & 200 & 100 & $N$ & 30 & $N$ & 500 & $N$ \\
\hline 435925 & 30 & 20 & 15 & $N$ & 200 & 100 & $N$ & 70 & N & 500 & $N$ \\
\hline 435926 & 30 & 20 & 15 & $\{10$ & 150 & 100 & $N$ & 50 & $N$ & 300 & $N$ \\
\hline 435927 & 30 & 20 & 15 & 20 & 200 & 100 & $N$ & 70 & $N$ & 500 & $\mathbf{N}$ \\
\hline 435928 & 30 & 30 & 15 & 10 & 200 & 100 & $N$ & 50 & $N$ & 700 & $N$ \\
\hline 435929 & 30 & 15 & 15 & 30 & 150 & 100 & N & 50 & $N$ & 500 & N \\
\hline 435930 & 50 & 30 & 20 & $N$ & 200 & 100 & N & 50 & $N$ & 500 & $N$ \\
\hline 435931 & 50 & 20 & 20 & $N$ & 300 & 150 & $N$ & 50 & N & 300 & $\mathrm{~N}$ \\
\hline 435932 & 30 & 20 & 10 & $\mathbf{N}$ & 150 & 100 & $N$ & 30 & $N$ & 300 & $N$ \\
\hline 435933 & 50 & 30 & 20 & $N$ & 200 & 150 & $N$ & 50 & $N$ & 300 & $N$ \\
\hline
\end{tabular}




\begin{tabular}{|c|c|c|c|c|c|c|c|c|c|}
\hline Sample & Latitude & Longitude & $\begin{array}{c}\text { Fe-pet. } \\
5\end{array}$ & $\underset{5}{M g-p c t .}$ & $\begin{array}{c}\text { Ca-pet. } \\
5\end{array}$ & $\begin{array}{c}\text { Ti-pct. } \\
5\end{array}$ & $\begin{array}{c}\text { Mn-pp } \\
5\end{array}$ & $\begin{array}{c}\mathrm{Ag}-p \mathrm{p} \\
5\end{array}$ & $\begin{array}{c}\text { As-ppm } \\
5\end{array}$ \\
\hline 435934 & 652948 & 1464548 & 5.00 & 1.50 & 1.5 & 1.000 & 700 & N & $N$ \\
\hline 435935 & 653059 & 1464349 & 5.00 & 1.50 & 1.0 & 1.000 & 700 & N & $N$ \\
\hline $43593 b$ & 653118 & 1464425 & 5.00 & 1.50 & 1.0 & 1.000 & 700 & $N$ & $N$ \\
\hline 435937 & 653159 & 1464534 & 5.00 & 2.00 & 2.0 & 1.000 & 1,000 & 2.0 & $N$ \\
\hline 435938 & 653231 & 1464215 & 5.00 & 1.50 & 1.0 & 1.000 & 700 & $H$ & $N$ \\
\hline 435939 & 653239 & 146438 & 5.00 & 1.50 & 1.0 & 1.000 & 1,000 & $N$ & N \\
\hline 435940 & 653312 & 1464311 & 5.00 & 1.50 & 1.0 & .700 & 1,000 & N & $N$ \\
\hline 435941 & 653345 & $14647 \quad 3$ & 5.00 & 1.50 & 1.5 & 1.000 & 1,500 & N & N \\
\hline 435942 & 653435 & 1464533 & 5.00 & 1.50 & 2.0 & 1.000 & 1,500 & .5 & $N$ \\
\hline 435944 & 653518 & 1464533 & 3.00 & 1.00 & 1.5 & .700 & 1,000 & .5 & N \\
\hline 435945 & $6536 \quad 6$ & $14 b \quad 4526$ & 5.00 & 1.50 & .5 & 1.000 & 1,500 & N & N \\
\hline 435947 & 653718 & 1464328 & 5.00 & 1.50 & 1.0 & .700 & 1,000 & $<.5$ & $H$ \\
\hline 435948 & 653732 & 146442 & 5.00 & 1.50 & 1.0 & 1.000 & 1,000 & 1.0 & $N$ \\
\hline 435949 & 653941 & 1464351 & 2.00 & 1.00 & 2.0 & .500 & 700 & $N$ & N \\
\hline 435950 & $6540 \quad 8$ & 1464439 & 5.00 & 1.50 & 1.0 & 1.000 & 1,000 & $<.5$ & $N$ \\
\hline 435951 & 65426 & 1464313 & 5.00 & 1.50 & 2.0 & 1.000 & 1,500 & N & $N$ \\
\hline 435952 & 654139 & $146 \quad 43 \quad 32$ & 5.00 & 2.00 & 2.0 & .700 & 2,000 & N & $N$ \\
\hline 435953 & 65449 & $146 \quad 46 \quad 13$ & 2.00 & .70 & 2.0 & .500 & 200 & N & $N$ \\
\hline 435954 & 653929 & 1463813 & 3.00 & 1.50 & 2.0 & 1.000 & 1,500 & N & $N$ \\
\hline 435955 & 653911 & 146380 & 5.00 & 1.50 & 2.0 & 1.000 & 1,500 & N & $N$ \\
\hline 435956 & 653633 & $14 b \quad 4023$ & 5.00 & 2.00 & 1.0 & 1.000 & 1,000 & 4.5 & $N$ \\
\hline 4.35957 & 653623 & 1463933 & 5.00 & 2.00 & 2.0 & 1.000 & 1,000 & $N$ & N \\
\hline 435958 & 653454 & $14 b 3921$ & 3.00 & 1.50 & 1.5 & 1.000 & 500 & $N$ & $N$ \\
\hline 435959 & 653456 & $14638 \quad 14$ & 5.00 & 1.50 & 1.0 & 1.000 & 500 & $N$ & N \\
\hline 435960 & $6534 \quad 1$ & 146393 & 5.00 & 1.50 & 1.5 & .700 & 700 & $N$ & $N$ \\
\hline 435961 & 653052 & $14640 \quad 5$ & 1.00 & .15 & .7 & .100 & 500 & $<.5$ & $N$ \\
\hline 435962 & 652836 & 1464233 & 3.00 & 1.00 & 1.0 & .500 & 500 & $N$ & $N$ \\
\hline 435963 & 652654 & $14642 \quad 1$ & 10.00 & 1.50 & 3.0 & 1.000 & 1,000 & $N$ & $N$ \\
\hline 435964 & 652617 & 1464158 & 7.00 & 1.50 & 3.0 & 1.000 & 1,000 & N & N \\
\hline 435965 & 652424 & 1463835 & 7.00 & 1.50 & 1.5 & 1.000 & 1,000 & N & $N$ \\
\hline 435966 & 652435 & 1463911 & 10.00 & 1.50 & 2.0 & 1.000 & 1,000 & $N$ & $N$ \\
\hline 435967 & 652224 & 1464028 & 1.50 & .30 & 2.0 & .200 & 700 & N & $N$ \\
\hline 435968 & 652053 & $14640 \quad 0$ & 10.00 & 1.50 & .3 & 1.000 & 1,000 & N & $N$ \\
\hline 435969 & 65183 & $\begin{array}{lll}146 & 40 & 17\end{array}$ & 10.00 & 2.00 & 3.0 & $\$ 1.000$ & 1,000 & N & $N$ \\
\hline 435970 & 651811 & $146 \quad 3252$ & 15.00 & 2.00 & 1.5 & 1.000 & 1,500 & $N$ & $N$ \\
\hline 435971 & 65190 & $146 \quad 2813$ & 10.00 & 2.00 & 2.0 & 1.000 & 1,000 & $N$ & $N$ \\
\hline 435972 & 652144 & $146 \quad 2925$ & 7.00 & 1.50 & 1.0 & 1.000 & 1,000 & $N$ & $N$ \\
\hline 435973 & 652133 & $146 \quad 28 \quad 18$ & 10.00 & 2.00 & 1.0 & 1.000 & 1,500 & N & $N$ \\
\hline 435974 & 652347 & 146256 & 7.00 & 1.50 & 1.0 & 1.000 & 1,000 & N & N \\
\hline 435975 & 652411 & $146 \quad 25 \quad 2$ & 7.00 & 1.50 & .7 & .700 & 1,500 & $N$ & H \\
\hline 435976 & $\begin{array}{lll}6524 & 1\end{array}$ & $14 b \quad 22 \quad b$ & 10.00 & 1.50 & 1.0 & 1.000 & 1,500 & N & N \\
\hline 435977 & 65244 & 1462146 & 10.00 & 2.00 & 2.0 & 1.000 & 2,000 & N & $N$ \\
\hline 435978 & 652657 & 1461941 & 7.00 & 1.50 & 2.0 & .700 & 3,000 & $N$ & N \\
\hline 435979 & 652739 & 1462015 & 5.00 & 1.50 & .5 & .700 & 2,000 & N & $N$ \\
\hline 435980 & 652723 & $146 \quad 2528$ & 7.00 & 2.00 & 1.0 & 1.000 & 2,000 & N & $N$ \\
\hline
\end{tabular}


TAELE 2.--FESULTS OF SPECTROGFAPHIC AMALYSES OF MURE STREAM-SEDIMENT SAMPLES FROM THE LIVENGOOD

AND WESTERH $1 / 3$ OF THE CIRCLE QUADRANGLES, ALASKA--Continued

\begin{tabular}{|c|c|c|c|c|c|c|c|c|c|c|}
\hline Sample & $\begin{array}{c}B-\rho \rho \text {. } \\
5\end{array}$ & $\begin{array}{c}\text { Ba-ppa } \\
5\end{array}$ & 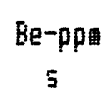 & $\begin{array}{c}B i-p p \\
5\end{array}$ & $\begin{array}{c}\text { Co-ppin } \\
5\end{array}$ & $\begin{array}{c}\mathrm{Cr}-\mathrm{pp} \text {. } \\
5\end{array}$ & $\begin{array}{c}\text { Cu-ppi } \\
5\end{array}$ & $\begin{array}{l}\text { La-ppi } \\
\quad \equiv\end{array}$ & $\begin{array}{c}\text { Mo-ppi } \\
5\end{array}$ & $\begin{array}{c}\text { Nb-pp } \\
5\end{array}$ \\
\hline 435934 & 100 & 1,000 & 1.0 & $N$ & 15 & 100 & 20 & 70 & $N$ & $<20$ \\
\hline 435935 & 150 & 700 & 5.0 & $H$ & 15 & 100 & 15 & 70 & $N$ & 20 \\
\hline 435936 & 150 & 700 & 1.5 & $N$ & 15 & 150 & 15 & 50 & 15 & 20 \\
\hline 435937 & 100 & 1,500 & 1.0 & $N$ & 15 & 150 & 20 & 50 & $\mathrm{~N}$ & 20 \\
\hline 435938 & 100 & 700 & 1.0 & $N$ & 15 & 100 & 15 & 70 & $N$ & $\langle 20$ \\
\hline 435939 & 100 & 1,000 & 2.0 & $N$ & 15 & 100 & 20 & 70 & $H$ & $<20$ \\
\hline 435940 & 100 & 1,500 & 1.0 & N & 15 & 100 & 20 & 70 & $N$ & $\langle 20$ \\
\hline 435941 & 100 & 1,500 & 3.0 & $N$ & 20 & 200 & 30 & 70 & $\langle 5$ & 20 \\
\hline 435942 & 70 & 1,000 & 15.0 & $N$ & 15 & 200 & 30 & 100 & 5 & 20 \\
\hline 435944 & 50 & 700 & 30.0 & 10 & 10 & 70 & 20 & 50 & $<5$ & $<20$ \\
\hline 435945 & 150 & 1,000 & 1.0 & $N$ & 15 & 100 & 20 & 50 & N & $<20$ \\
\hline 435947 & 100 & 1,000 & 5.0 & $\langle 10$ & 10 & 100 & 20 & 200 & $<5$ & 20 \\
\hline 435948 & 70 & 1,000 & 10.0 & $<10$ & 15 & 100 & 20 & 100 & $<5$ & $<20$ \\
\hline 435949 & 50 & 700 & 7.0 & $H$ & 7 & 50 & 15 & 100 & $<5$ & $N$ \\
\hline 435950 & 200 & 700 & 2.0 & $H$ & 15 & 70 & 20 & 50 & $N$ & $<20$ \\
\hline 435951 & 100 & 1,500 & 3.0 & $\mathbf{N}$ & 20 & 100 & 30 & 100 & $\mathrm{~N}$ & 20 \\
\hline 435952 & 100 & 1,500 & 3.0 & $\mathrm{~N}$ & 20 & 100 & 50 & 100 & $N$ & $<20$ \\
\hline 435953 & 30 & 1,000 & $(1.0$ & $\mathrm{N}$ & N & 50 & 15 & $N$ & N & $N$ \\
\hline 435954 & 100 & 1,500 & 5.0 & $N$ & 15 & 100 & 30 & 200 & N & 20 \\
\hline 435955 & 70 & 1,500 & 11.0 & $N$ & 20 & 150 & 20 & 70 & N & $N$ \\
\hline 435956 & 100 & 1,500 & 2.0 & $N$ & 20 & 200 & 50 & 100 & $N$ & $<20$ \\
\hline 435957 & 100 & 1,500 & 2.0 & $N$ & 15 & 150 & 30 & 200 & $N$ & 20 \\
\hline 435958 & 70 & 1,000 & 1.0 & H & 15 & 100 & 20 & 100 & $N$ & $<20$ \\
\hline 435959 & 100 & 1,000 & 1.0 & $N$ & 15 & 100 & 20 & 70 & $N$ & $<20$ \\
\hline 435960 & 50 & 700 & 1.5 & $N$ & 10 & 100 & 15 & 30 & $N$ & $N$ \\
\hline 435961 & 10 & 300 & 1.5 & $N$ & $N$ & 20 & 20 & $N$ & $N$ & H \\
\hline 435962 & 70 & 700 & 1.5 & H & 10 & 50 & 15 & 30 & $N$ & $N$ \\
\hline 435963 & 100 & 1,000 & 1.5 & $N$ & 20 & 200 & 30 & 70 & H & 20 \\
\hline 435964 & 150 & 1,000 & 1.0 & $N$ & 15 & 150 & 20 & 70 & $N$ & 20 \\
\hline 435965 & 150 & 1,000 & 2.0 & $N$ & 15 & 150 & 20 & 50 & $H$ & $\langle 20$ \\
\hline 435966 & 150 & 1,500 & 1.5 & $N$ & 20 & 150 & 20 & 70 & $N$ & 20 \\
\hline 435967 & 20 & 500 & 1.0 & $N$ & $N$ & 20 & 10 & 50 & N & $N$ \\
\hline 435968 & 200 & 1,000 & 1.5 & $N$ & 15 & 100 & 20 & 50 & H & $<20$ \\
\hline 435969 & 70 & 700 & $\langle 1.0$ & $N$ & 20 & 150 & 70 & 30 & $N$ & $N$ \\
\hline 435970 & 100 & 1,500 & 1.0 & $N$ & 30 & 200 & 70 & 50 & $N$ & $<20$ \\
\hline 435971 & 100 & 700 & 41.0 & $N$ & 20 & 150 & 50 & 30 & $N$ & $N$ \\
\hline 435972 & 100 & 1,000 & 1.0 & $H$ & 15 & 70 & 15 & 70 & $N$ & $<20$ \\
\hline 435973 & 200 & 1,500 & 1.0 & $\mathrm{~N}$ & 20 & 150 & 30 & 70 & H & $<20$ \\
\hline 435974 & 150 & 1,000 & 1.5 & N & 20 & 100 & 30 & 70 & $\ddot{n}$ & $<20$ \\
\hline 435975 & 300 & 1,000 & 2.0 & $N$ & 10 & 50 & 15 & 50 & $N$ & $<20$ \\
\hline 435976 & 200 & 1,000 & 2.0 & $N$ & 15 & 100 & 20 & 50 & N & 20 \\
\hline 435977 & 200 & 1,500 & 1.5 & $N$ & 20 & 200 & 20 & 50 & $<5$ & 20 \\
\hline 4.35978 & 50 & 1,500 & 1.0 & $N$ & 20 & 150 & 15 & 70 & N & $N$ \\
\hline 435979 & 200 & 700 & 7.0 & $n$ & 15 & 100 & 15 & 70 & $N$ & 20 \\
\hline 435980 & 100 & 1,000 & 5.0 & $n$ & 15 & 150 & 30 & 70 & N & $<20$ \\
\hline
\end{tabular}


TABLE 2. - RESULTS OF SPECTRDGRAPHIC AMALYSES OF NURE STREAM-SEDIMENT SAMPLES FROM THE LIVEMGDDD

AND WESTERN $1 / 3$ OF THE CIRCLE QUADRANGLES, ALASKA--Continued

\begin{tabular}{|c|c|c|c|c|c|c|c|c|c|c|c|}
\hline Sample & $\begin{array}{c}\mathrm{Ni}-\mathrm{ppm} \\
5\end{array}$ & $\begin{array}{c}\text { Fb-pp再 } \\
5\end{array}$ & $\begin{array}{c}S c-p p \text { 而 } \\
5\end{array}$ & $\begin{array}{c}S n-p p \text { i } \\
5\end{array}$ & $\begin{array}{c}\mathrm{Sr}-p p m \\
5\end{array}$ & $\begin{array}{c}V-p p m i t \\
5\end{array}$ & $\begin{array}{c}\text { W-ppi } \\
5\end{array}$ & $\begin{array}{c}y-p p q \\
5\end{array}$ & $\begin{array}{c}\ln -p \text { pm } \\
5\end{array}$ & $\begin{array}{c}2 r \text {-ppmi } \\
5\end{array}$ & $\begin{array}{c}\text { Th-pp田 } \\
5\end{array}$ \\
\hline 435934 & 30 & 20 & 20 & $N$ & 200 & 100 & $N$ & 50 & $n$ & 300 & $N$ \\
\hline 435935 & 30 & 20 & 15 & $N$ & 150 & 100 & $N$ & 70 & $N$ & 500 & $n$ \\
\hline 435936 & 30 & 30 & 20 & 10 & 200 & 100 & $N$ & 50 & $N$ & 500 & $N$ \\
\hline 435937 & 30 & 30 & 20 & $N$ & 200 & 150 & $N$ & 50 & $N$ & 300 & $N$ \\
\hline 435938 & 30 & 20 & 20 & $N$ & 200 & 100 & $H$ & 30 & $N$ & 500 & H \\
\hline 435939 & 30 & 50 & 20 & 15 & 150 & 100 & $H$ & 70 & $N$ & 300 & $N$ \\
\hline 435940 & 30 & 30 & 20 & $N$ & 150 & 100 & $N$ & 50 & $N$ & 300 & $N$ \\
\hline 435941 & 50 & 30 & 30 & $n$ & 200 & 150 & $N$ & 70 & $N$ & 700 & $N$ \\
\hline 435942 & 50 & 100 & 20 & 15 & 200 & 150 & N & 100 & 500 & 700 & $<100$ \\
\hline 435944 & 20 & 100 & 15 & $<10$ & 100 & 100 & $N$ & 70 & 300 & 300 & $<100$ \\
\hline 435945 & 30 & 30 & 15 & $N$ & 150 & 70 & $N$ & 50 & $N$ & 300 & $H$ \\
\hline 435947 & 20 & 50 & 20 & 70 & 100 & 100 & $N$ & 150 & $N$ & 1,000 & 100 \\
\hline 435948 & 30 & 70 & 20 & 10 & 200 & 100 & $N$ & 100 & 200 & 300 & $\$ 100$ \\
\hline 435949 & 20 & 20 & 15 & $H$ & 200 & 70 & $N$ & 70 & $N$ & 200 & $N$ \\
\hline 435950 & 20 & 50 & 15 & 15 & 150 & 70 & $N$ & 50 & $N$ & 700 & $N$ \\
\hline 435951 & 30 & 70 & 20 & $N$ & 200 & 100 & $N$ & 100 & $N$ & 700 & $H$ \\
\hline 435952 & 30 & 70 & 20 & 10 & 200 & 100 & $N$ & 100 & $N$ & 500 & N \\
\hline 435953 & 15 & 10 & 10 & $N$ & 200 & 70 & $N$ & 20 & $N$ & 150 & $N$ \\
\hline 435954 & 30 & 70 & 20 & $<10$ & 200 & 100 & $N$ & 100 & $N$ & 500 & $(100$ \\
\hline 435955 & 50 & 20 & 20 & $N$ & 200 & 100 & $N$ & 50 & $N$ & 200 & $N$ \\
\hline 435956 & 50 & 100 & 30 & 10 & 100 & 100 & $H$ & 100 & 200 & 300 & $N$ \\
\hline 435957 & 30 & 100 & 20 & $N$ & 200 & 100 & $N$ & 100 & $<200$ & 700 & $N$ \\
\hline 435958 & 30 & 20 & 20 & $n$ & 150 & 100 & $N$ & 50 & $N$ & 300 & $N$ \\
\hline 435959 & 30 & 30 & 20 & $N$ & 150 & 100 & N & 30 & $N$ & 300 & $N$ \\
\hline 435960 & 30 & 20 & 10 & $N$ & 150 & 100 & $N$ & 50 & N & 200 & $N$ \\
\hline 435961 & 20 & 10 & 7 & $N$ & $N$ & 70 & $N$ & 20 & $N$ & 70 & $N$ \\
\hline 435962 & 20 & 15 & 10 & $N$ & 100 & 100 & $N$ & 20 & $N$ & 150 & $N$ \\
\hline 435963 & 50 & 30 & 20 & $H$ & 300 & 150 & N & 50 & $N$ & 300 & $N$ \\
\hline 435964 & 30 & 30 & 15 & $<10$ & 200 & 100 & $N$ & 70 & $N$ & 500 & $<100$ \\
\hline 435965 & 30 & 30 & 15 & 15 & 150 & 100 & $N$ & 70 & $N$ & 300 & $N$ \\
\hline 4.35966 & 30 & 20 & 20 & $\$ 10$ & 200 & 150 & $H$ & 70 & $\mathrm{~N}$ & 500 & $N$ \\
\hline 435967 & 10 & $<10$ & 7 & $N$ & 100 & 50 & $N$ & 50 & $N$ & 200 & $N$ \\
\hline 435968 & 30 & 20 & 15 & $N$ & 150 & 100 & $N$ & 30 & $N$ & 500 & $N$ \\
\hline 435969 & 50 & 15 & 30 & $N$ & 150 & 300 & $N$ & 50 & $N$ & 500 & $N$ \\
\hline 435970 & 50 & 30 & 30 & $N$ & 200 & 300 & $N$ & 50 & N & 500 & $N$ \\
\hline 435971 & 30 & 20 & 30 & $N$ & 100 & 150 & $N$ & 30 & $N$ & 500 & $N$ \\
\hline 435972 & 30 & 20 & 15 & $N$ & 150 & 100 & $N$ & 30 & $H$ & 500 & $N$ \\
\hline 435973 & 50 & 50 & 20 & $N$ & 200 & 150 & $N$ & 50 & N & 500 & $N$ \\
\hline 435974 & 50 & 30 & 20 & $N$ & 200 & 150 & $N$ & 50 & $N$ & 500 & $N$ \\
\hline 435975 & 20 & 30 & 20 & 70 & 150 & 100 & $N$ & 30 & $H$ & 300 & $N$ \\
\hline 435976 & 20 & 30 & 15 & 50 & 200 & 100 & $N$ & 70 & $N$ & 700 & $N$ \\
\hline 435977 & 30 & 50 & 20 & 10 & 200 & 150 & $N$ & 50 & $N$ & 500 & $N$ \\
\hline 435978 & 30 & 30 & 20 & $<10$ & 200 & 150 & $N$ & 30 & $N$ & 300 & $N$ \\
\hline 435979 & 20 & 20 & 15 & 50 & 150 & 70 & $N$ & 100 & N & 500 & $N$ \\
\hline 435980 & 50 & 30 & 20 & 15 & 200 & 100 & $N$ & 50 & $N$ & 300 & $N$ \\
\hline
\end{tabular}


TABLE 2. --RESULTS OF SPECTRDGRAPHIC ANALYSES OF NURE STREAM-SEDIMENT SAMPLES FROM THE LIVENGDDD AND WESTERN $1 / 3$ OF THE CIRCLE QUADRANGLES, ALASKA--ContinuRd

\begin{tabular}{|c|c|c|c|c|c|c|c|c|c|}
\hline Sample & Latitude & Longitude & $\begin{array}{c}\text { Fe-pct. } \\
5\end{array}$ & $\begin{array}{c}\text { Mg-pct. } \\
s\end{array}$ & $\begin{array}{c}\text { Ca-pct. } \\
\text { s }\end{array}$ & $\begin{array}{c}\text { Ti-pct. } \\
s\end{array}$ & $\begin{array}{l}\text { Hn-ppa } \\
5\end{array}$ & $\begin{array}{c}\text { Ag-ppa } \\
s\end{array}$ & $\begin{array}{c}\text { As-ppi } \\
5\end{array}$ \\
\hline 435981 & $6529 \quad 9$ & 1462342 & 7.00 & 1.50 & .5 & $>1.000$ & 1,000 & $N$ & $N$ \\
\hline 435982 & 652925 & 1462455 & 10.00 & 2.00 & .5 & 1.000 & 2,000 & $N$ & $N$ \\
\hline 435983 & 653038 & $146 \quad 2049$ & 7.00 & 1.50 & .5 & $>1.000$ & 1,500 & $N$ & $N$ \\
\hline 435984 & 653015 & $146 \quad 2041$ & 7.00 & 1.50 & .7 & 1.000 & 2,000 & $N$ & $N$ \\
\hline 435985 & 653111 & 146225 & 7.00 & 1.50 & 1.0 & .700 & 1,500 & $N$ & N \\
\hline 435986 & 65314 & 1462344 & 7,00 & 1.50 & .5 & 31.000 & 1,500 & $N$ & $N$ \\
\hline 435987 & 652639 & 1461416 & 7.00 & 1.50 & .5 & $>1.000$ & 1,500 & $N$ & $N$ \\
\hline 435988 & 652528 & $14614 \quad 3$ & 3.00 & 1.00 & .7 & .700 & 500 & $N$ & $N$ \\
\hline 435989 & 652522 & $146 \quad 1520$ & 10.00 & 1.50 & .3 & 1.000 & 1,500 & $N$ & $N$ \\
\hline 435990 & 652441 & $146 \quad 12 \quad 42$ & 7.00 & 1.50 & .7 & 1.000 & 2,000 & N & $N$ \\
\hline 435991 & $6524 \quad 7$ & 1461546 & 7.00 & 2.00 & 1.0 & 1.000 & 2,000 & 5.0 & $N$ \\
\hline 435992 & 652356 & $14617 \quad 8$ & 10.00 & 2,00 & 1.5 & 1.000 & 1,500 & $N$ & $N$ \\
\hline 435993 & 652128 & 1462029 & 10.00 & 2.00 & .7 & 1.000 & 1,000 & N & $N$ \\
\hline 435994 & $3522 \quad 4$ & 1462044 & 7.00 & 1.50 & 2.0 & 1.000 & 1,000 & $\ddot{N}$ & $N$ \\
\hline 435995 & 652137 & 1461654 & 7.00 & 1.50 & 2.0 & 1.000 & 1,500 & $N$ & $N$ \\
\hline 435996 & 652129 & 146934 & 7.00 & 2.00 & .5 & 1.000 & 1,500 & $N$ & $N$ \\
\hline 435997 & 651833 & 1461729 & 7.00 & 2.00 & 1.0 & 1.000 & 1,000 & $N$ & $N$ \\
\hline 435998 & $6519 \quad 4$ & 1462148 & 10.00 & 2.00 & 2.0 & 1.000 & 1,500 & $N$ & $N$ \\
\hline 435999 & 651848 & 1462115 & 7.00 & 2.00 & .7 & .700 & 2,000 & N & $N$ \\
\hline 436000 & 651648 & $146 \quad 35 \quad 17$ & 5.00 & 1.50 & 1.0 & 1.000 & 1,000 & .5 & $N$ \\
\hline
\end{tabular}


TABLE 2, - RESULTS OF SPECTRDgRaPHIC ANALYSES OF NURE STREAM-SEDIMENT SAMPLES FROM THE LIVENGDOD AND WESTERN $1 / 3$ OF THE CIRCLE QUADRANGLES, ALASKA--Continued

\begin{tabular}{|c|c|c|c|c|c|c|c|c|c|c|}
\hline Satiple & $\begin{array}{c}\text { B-ppo } \\
\vdots\end{array}$ & $\begin{array}{c}\text { Ba-ppi } \\
5\end{array}$ & $\begin{array}{c}\text { Be-ppin } \\
5\end{array}$ & $\begin{array}{c}\text { Bi-ppi } \\
5\end{array}$ & $\begin{array}{c}\text { Co-ppo } \\
5\end{array}$ & $\begin{array}{c}C r-p \rho \text { il } \\
5\end{array}$ & $\begin{array}{c}\text { Cu-ppon } \\
5\end{array}$ & $\begin{array}{c}\text { La-pofil } \\
z\end{array}$ & $\begin{array}{c}\text { Mo-pp四 } \\
5\end{array}$ & $\begin{array}{c}\text { Nb-ppl } \\
s\end{array}$ \\
\hline 435981 & 200 & 700 & 2.0 & $N$ & 15 & 150 & 20 & 50 & $<5$ & 20 \\
\hline 435982 & 70 & 1,500 & 1.0 & $N$ & 30 & 150 & 50 & 50 & N & $<20$ \\
\hline 435983 & 200 & 1,000 & 2.0 & N & 15 & 50 & 20 & 50 & $N$ & 20 \\
\hline 435984 & 70 & 700 & 1.0 & $N$ & 10 & 70 & 15 & 30 & $N$ & $<20$ \\
\hline 435985 & 150 & 1,000 & 2.0 & $N$ & 15 & 100 & 20 & 70 & $N$ & $N$ \\
\hline 435986 & 100 & 700 & 1.0 & $N$ & 15 & 100 & 20 & 50 & $N$ & 20 \\
\hline 435987 & 150 & 700 & $\langle 1.0$ & $N$ & 15 & 70 & 20 & 30 & $N$ & 20 \\
\hline 435988 & 70 & 700 & 1.0 & $N$ & 7 & 70 & 20 & 50 & 15 & N \\
\hline 435989 & 150 & 1,000 & 1.0 & $N$ & 20 & 70 & 220 & 30 & $N$ & $<20$ \\
\hline 435990 & 150 & 1,500 & $\ll 1.0$ & $N$ & 20 & 100 & 20 & 70 & $N$ & $N$ \\
\hline 435991 & 100 & 1,000 & 1.5 & $N$ & 20 & 70 & 50 & 30 & $N$ & H \\
\hline 435992 & 100 & 1,000 & 1.0 & $N$ & 30 & 150 & 50 & 50 & N & 120 \\
\hline 435993 & 150 & 1,500 & 1.0 & $N$ & 20 & 200 & 50 & 50 & $N$ & 20 \\
\hline 435994 & 150 & 1,000 & 1.5 & $N$ & 15 & 150 & 20 & 50 & $N$ & 20 \\
\hline 435995 & 100 & 1,000 & 1.0 & $N$ & 20 & 200 & 30 & 50 & $N$ & $<20$ \\
\hline 435996 & 150 & 1,000 & 1.0 & $H$ & 20 & 150 & 30 & 50 & $N$ & 20 \\
\hline 435997 & 150 & 700 & 1.0 & $N$ & 20 & 150 & 50 & 50 & $\mathrm{~N}$ & 20 \\
\hline 435998 & 100 & 700 & 1.0 & $N$ & 20 & 200 & 50 & 50 & $N$ & $H$ \\
\hline 435999 & 100 & 1,000 & 1.0 & $N$ & 20 & 200 & 30 & 50 & $N$ & $N$ \\
\hline 436000 & 100 & 1,000 & 1.0 & $N$ & 15 & 100 & 30 & 50 & N & 20 \\
\hline
\end{tabular}


TABLE 2.--RESUL TS OF SPECTROGRAPHIC AMALYSES OF NURE STREAM-SEDIMENT SAMPLES FROM THE LIVENGODD

AND WESTERN $1 / 3$ OF THE CIRCLE QUADRAMGLES, ALASKA--ContinUEd

\begin{tabular}{|c|c|c|c|c|c|c|c|c|c|c|c|}
\hline Sampie & $\begin{array}{c}\mathrm{Ni}-\mathrm{pp} \text { i } \\
5\end{array}$ & $\begin{array}{c}\mathrm{Pb}-p p a \\
\equiv\end{array}$ & $\begin{array}{l}\text { Sc-ppm } \\
\quad 5\end{array}$ & $\begin{array}{c}S n-p p \text { m } \\
5\end{array}$ & $\begin{array}{c}5 r-p p \\
s\end{array}$ & $\begin{array}{c}V \text {-pp } \\
s\end{array}$ & $\begin{array}{c}H-p p h \\
s\end{array}$ & $\begin{array}{c}Y-p p \text { it } \\
5\end{array}$ & $\begin{array}{c}2 n-p \text { pi } \\
s\end{array}$ & $\begin{array}{c}\text { Zr-pp } \\
5\end{array}$ & $\begin{array}{c}\text { Th-pp } \\
5\end{array}$ \\
\hline 435981 & 30 & 50 & 10 & 30 & 150 & 100 & $N$ & 100 & $N$ & 700 & $N$ \\
\hline 435982 & 50 & 50 & 20 & $N$ & 150 & 200 & $N$ & 50 & $H$ & 500 & N \\
\hline 435983 & 20 & 30 & 15 & 110 & 200 & 70 & $N$ & 70 & $N$ & 700 & $N$ \\
\hline 435984 & 20 & 30 & 10 & $N$ & 150 & 100 & $N$ & 50 & $N$ & 500 & $N$ \\
\hline 435985 & 30 & 20 & 15 & $N$ & 200 & 100 & N & 50 & $N$ & 300 & $N$ \\
\hline 435996 & 30 & 30 & 15 & $N$ & 150 & 100 & $N$ & 50 & $N$ & 700 & H \\
\hline 435987 & 30 & 20 & 10 & $N$ & 150 & 70 & H & 50 & $N$ & 1,000 & $N$ \\
\hline 435988 & 20 & 30 & 15 & $N$ & $\ll 100$ & 150 & $N$ & 50 & $N$ & 500 & N \\
\hline 435989 & 30 & 30 & 10 & $N$ & 200 & 100 & $N$ & 30 & $N$ & 500 & $N$ \\
\hline 435990 & 30 & 30 & 15 & $N$ & 300 & 100 & $N$ & 150 & N & 200 & $N$ \\
\hline 435991 & 30 & 50 & 15 & $N$ & 150 & 150 & $N$ & 50 & $N$ & 500 & $N$ \\
\hline 435992 & 50 & 30 & 20 & $N$ & 200 & 200 & $N$ & 50 & $N$ & 500 & $\mathrm{~N}$ \\
\hline 435993 & 50 & 30 & 15 & $N$ & 200 & 150 & $N$ & 50 & $N$ & 300 & $N$ \\
\hline 435994 & 30 & 30 & 20 & $N$ & 200 & 150 & $N$ & 50 & $N$ & 1,000 & $N$ \\
\hline 435995 & 30 & 30 & 15 & $N$ & 200 & 200 & $N$ & 50 & $N$ & 700 & $N$ \\
\hline 435996 & 30 & 50 & 20 & 10 & 150 & 150 & $N$ & 50 & $N$ & 500 & $N$ \\
\hline 435997 & 30 & 50 & 15 & $N$ & 150 & 150 & $N$ & 50 & $\ddot{N}$ & 700 & $\mathrm{~N}$ \\
\hline 435998 & 50 & 20 & 20 & $N$ & 300 & 150 & $N$ & 50 & N & 300 & N \\
\hline 435999 & 50 & 30 & 20 & $\mathrm{~N}$ & 200 & 100 & $N$ & 50 & $N$ & 200 & $\mathrm{~N}$ \\
\hline 436000 & 50 & 20 & 20 & $N$ & 150 & 100 & $N$ & 30 & $N$ & 500 & $N$ \\
\hline
\end{tabular}

